\title{
The Effect of Genetic Background and Environment on Plasmid Fitness and Persistence
}

\author{
by
}

\author{
Amanda Christine Carroll
}

A thesis submitted to the Faculty of Graduate and Postdoctoral Affairs in partial fulfillment of the requirements for the degree of

Doctor of Philosophy

in

Biology

Carleton University

Ottawa, Ontario

(C) 2021, Amanda Christine Carroll 


\section{Abstract}

Antimicrobial resistance (AMR) is a major threat to public health, which, left unchecked, will have severe impacts on mortality. Plasmids are extrachromosomal DNA elements often capable of self-transmission to new hosts, and often carry AMR determinants. They are frequently deleterious and thus predicted to be lost to purifying selection. However, compensatory evolution, selection, and conjugation of the plasmid can stabilize it within populations, thus helping maintain AMR genes. In this thesis, I assess how bacterial hostplasmid fitness informs the strategies used for plasmid maintenance, compensatory evolution, and parallel evolution in a short- and long-term evolution experiment, as well as the impact of environment on plasmid fitness and resistance. In Chapter 3, I transferred the AMR plasmid pPB29 into six E. coli hosts and measured fitness before evolving each host-plasmid pair for 100 generations without antibiotic selection for the plasmid. I show that conjugation and compensatory evolution both contribute to plasmid maintenance, as conjugation of costly plasmids in mixed culture was frequent, and compensatory evolution was observed occasionally in monoculture. This shows the importance of fitness in determining how plasmids might be maintained within populations. In Chapter 4, I show that the fitness of three $E$. coli hosts carrying pPB29 increased over 500 generations following selection in varied concentrations of antibiotic, though fitness between cells evolved in those conditions did not vary significantly. I observed parallel evolution from phenotype to nucleotide level changes, with many transcription/translation-related mutations observed, suggesting a potential mechanism for the costliness of pPB29. Finally, in Chapter 5, I constructed six host-plasmid pairs with plasmids encoding either kanamycin or carbapenem resistance. I measured their 
fitness in twelve discrete environments where I varied three factors: the presence/absence of oxygen, $\mathrm{pH}$, and glucose concentration. I found that gene-by-environment interactions were important determinants for fitness, and that environment can mediate resistance in these strains. These results highlight the importance of studying plasmids in a more integrated way, since various factors, like starting fitness, level of plasmid selection, and environment can all act to shape the trajectory of plasmid-carrying strains, and, ultimately, the threat they pose in the fight against AMR. 


\section{Acknowledgements}

First and foremost, I would like to express my most sincere gratitude and thanks to my supervisor, Dr. Alex Wong. Alex: I applied for grad school right near the deadline in August 2016, and somehow, with a little luck, you were able to take me on in your lab. I will forever appreciate this opportunity that you've given me. Along the way, you've been an amazing mentor, and sometimes I can't believe what a kind, dedicated, and knowledgeable human you are. I've been through a lot of personal stumbling blocks and victories along the way and knowing that you've been there to guide me during this $\mathrm{PhD}$, with compassion, kindness, and a little humour, has been invaluable. Words will never be able to convey how glad I am to have worked for you these past six years, both during my undergraduate honours project and my graduate work. I think it's safe to say that this $\mathrm{PhD}$ has changed my life in ways I had never even anticipated. Thank you.

To my advisory committee, Dr. Burton Blais and Dr. Alexandre Poulain, thank you for all your comments and feedback along this journey. Your guidance has helped shape my research in ways I hadn't expected, and I will always appreciate that. Dr. Aaron Hinz - you're a science rockstar. Thanks for all of the advice, the help with strains, and being willing to chat about science in the lab. I'd also like to thank Suzanne Paterson and Sabrina Dawson for being awesome lab coordinators. I've had the best time TAing for both of you, and I'm glad to have been able to get to know you during my time at Carleton. To Ms. Shane and Ms. Erdelyi - both of you ignited my love of science and biology in high school. Grade 9 Science with Ms. Shane really made me think that science was something I wanted to be immersed in for my whole life, and Grade 11 and 12 Biology with Ms. Erdelyi narrowed my focus - you two helped start me on this amazing journey.

I would also like to thank everyone who has been on this journey with me, especially those in the Wong Lab family. All of you have made this $\mathrm{PhD}$ an exciting time, and I've experienced so many memorable things I never thought I would over the last five years. I've got to spend time with you all outside of the lab, whether it was at the pub after a conference or defence, weekend get-togethers, or the annual Wong Lab BBQ, and it's made getting through this $\mathrm{PhD}$ that much better. Bryn and Leah - you two were my first grad school friends, and I'm so glad I got to start this journey with you two back 
in 2016 - who knew grad school might be, dare I say, fun? CSM Waterloo would not have been half as great without the two of you. Katie and Laura, you guys got to learn firsthand how much I could talk in the lab or the office - thanks for always being up for a chat. Cat - lab work is hard but having a friend to get through it with makes it so much better, especially during a pandemic. Remy - who knew a butterfly show could shake things up so much? My Starbucks habit is thanks to you.

Mom and Dad: thank you for supporting me during this and everything else I've done in my life. I am incredibly blessed to be your daughter, and I don't know if I could have done this without you both. Dad: you encouraged me to go for it, and I will always be grateful for that. Mom: with the amount of time, you've listened to me talk about my research, you probably deserve an honorary $\mathrm{PhD}$. Thank you for always encouraging me to follow my dreams and for making sure I sometimes got away from my laptop to do something fun. To my brother Jacob, you've made me laugh more than anyone during the last five years and I couldn't have asked for a better younger brother. And to the rest of my family - you might not have always known exactly what I was up to for the last few years, but you all cheered me on along the way no matter what. This is for all of you. 


\section{Statement of Contribution}

The thesis, "The Effect of Genetic Background and Environment on Plasmid Fitness and Persistence" is comprised of a review paper and three studies.

Chapter 2, "Plasmid persistence: costs, benefits, and the plasmid paradox", is a review paper highlighting the main theory and results in the literature about plasmid maintenance. This was written by me with contributions from Dr. Alex Wong. This is a copy of the following paper: Carroll and Wong. 2018. Plasmid persistence: costs, benefits, and the plasmid paradox. Can. J. Microbiol. 64:293-304. This paper has been reformatted for this thesis.

Chapter 3, "Host-plasmid interactions in Escherichia coli and their effect on plasmid maintenance", is a result of experiments designed by myself with contributions from Dr. Alex Wong, with all experiments carried out by me. Dr. Aaron Hinz provided the protocol and vectors for the deletion of $l a c A$ and provided additional lacA-YFP strains to construct additional host-plasmid combinations. The manuscript was written by me with contributions and guidance by Dr. Alex Wong. A version of this chapter has been submitted to Evolution for review.

Chapter 4, "Effect of Variable Long-Term Selection on Plasmid Maintenance and Parallel Evolution in Escherichia coli", is the result of experiments primarily designed and carried out by me with guidance from Dr. Alex Wong.

Chapter 5, "Gene x Environment interactions in plasmid-carrying E. coli", is a result of experiments primarily designed and carried out by me with contributions from Dr. Alex Wong. 


\section{Table of Contents}

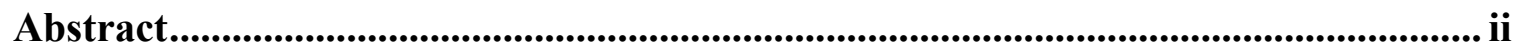

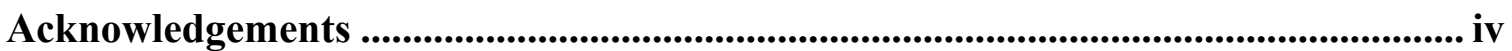

Statement of Contribution ...................................................................................... vi

Table of Contents .......................................................................................................................... vii

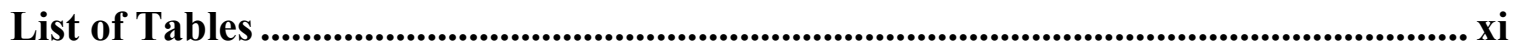

List of Figures................................................................................................................................... xiii

List of Appendices...................................................................................................................... xvi

Chapter 1: General Introduction ................................................................................... 1

$1.1 \quad$ Antibiotic Resistance ...................................................................................

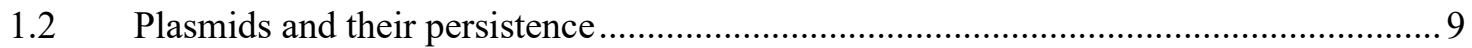

1.3 Genotype x Environment Interactions in Plasmid Maintenance ……………………....16

1.4 Parallel Evolution as a Tool for Understanding Host-Plasmid Co-Evolution ...............20

$1.5 \quad$ Major Themes and Questions ............................................................................2

1.5.1 Chapter 3 Questions and Hypotheses................................................................22

1.5.2 Chapter 4 Questions and Hypotheses........................................................................2

1.5.3 Chapter 5 Questions and Hypotheses.................................................................2

1.5.4 Significance of Research...........................................................................................2

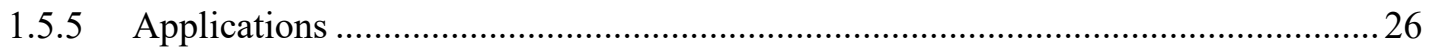

Chapter 2: Plasmid persistence: costs, benefits, and the plasmid paradox ............... 29

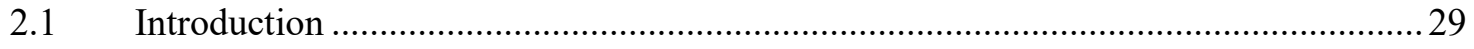

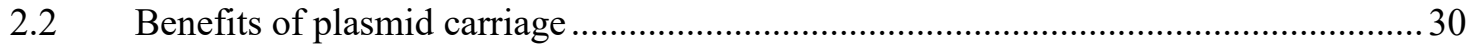

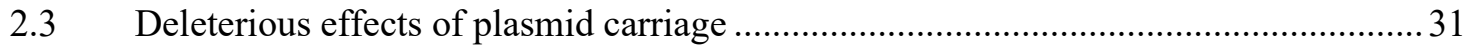

2.4 Mechanisms underlying the cost of plasmid carriage............................................... 36 


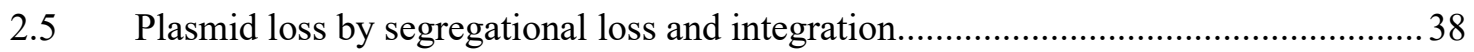

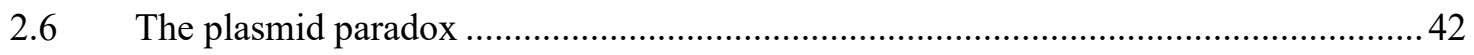

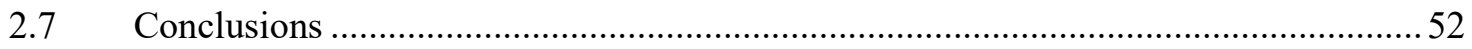

\section{Chapter 3: Host-plasmid interactions in Escherichia coli and their effect on plasmid}

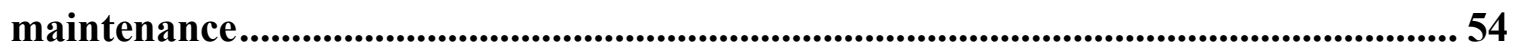

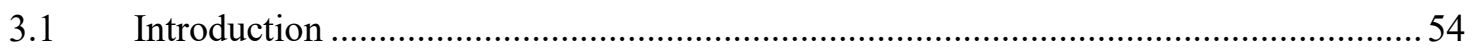

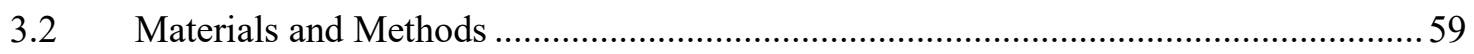

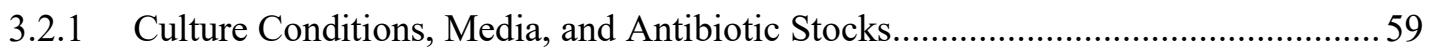

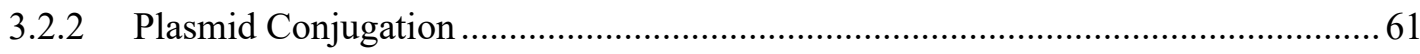

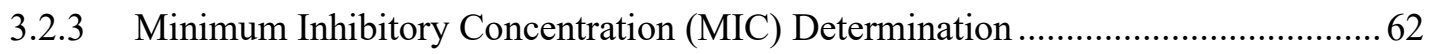

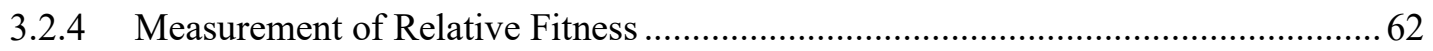

3.2.5 Selection Experiment and Plasmid Loss Enumeration............................................63

3.2.6 Mixed-Culture Selection in the Absence of Antibiotic ..............................................6 64

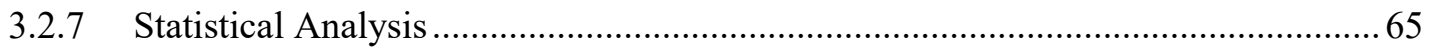

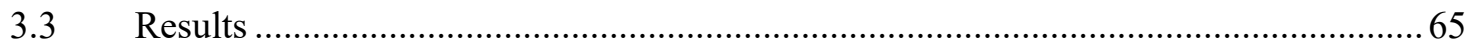

3.3.1 Genetic Background Effects on Plasmid Carriage …............................................... 65

3.3.2 Changes in Fitness and Resistance Following Selection in the Absence of

Antibiotic. .68

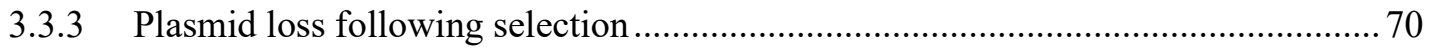

3.3.4 Plasmid dynamics following mixed-culture selection...................................... 71

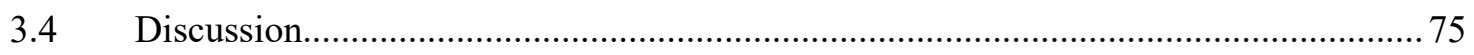

\section{Chapter 4: Effect of Variable Long-Term Selection on Plasmid Maintenance and}

Parallel Evolution in Escherichia coli ................................................................. 82

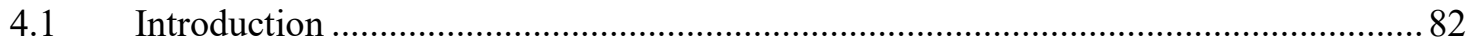

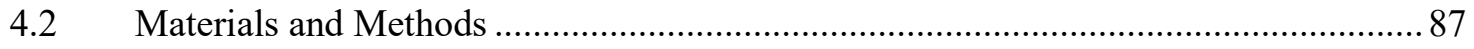

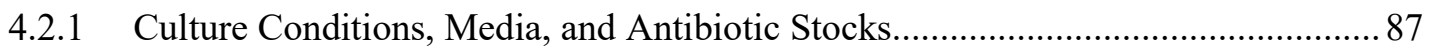




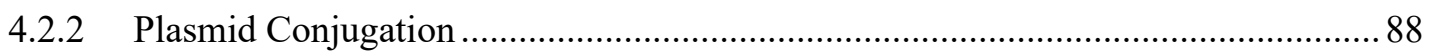

4.2.3 Selection Experiment and Plasmid Loss Enumeration........................................... 89

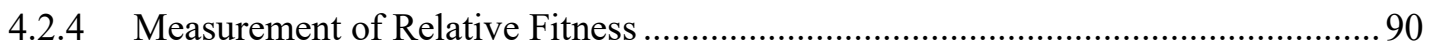

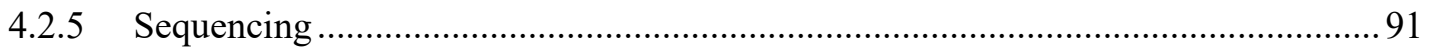

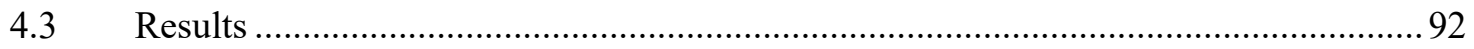

4.3.1 Fitness of Evolved Populations in the Absence of Antibiotic Selection ..................92

4.3.2 Fitness of Evolved Populations in Three Competition Environments ..................... 94

4.3.3 Plasmid Loss Following Experimental Evolution............................................... 101

4.3.4 Evidence of Parallel and Compensatory Evolution.............................................. 103

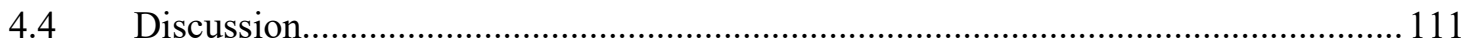

Chapter 5: Gene $x$ Environment interactions in plasmid-carrying $E$. coli............. 121

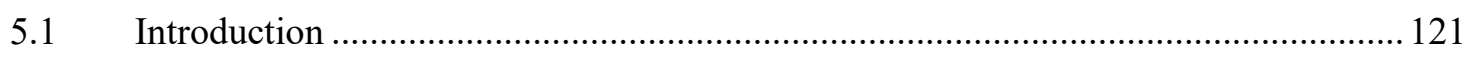

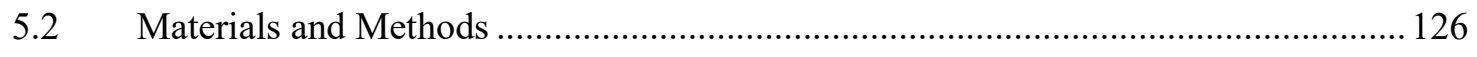

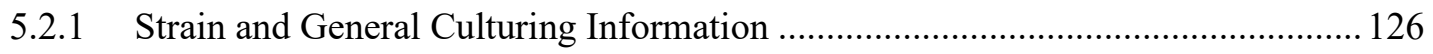

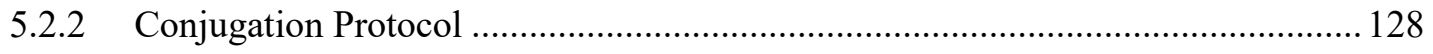

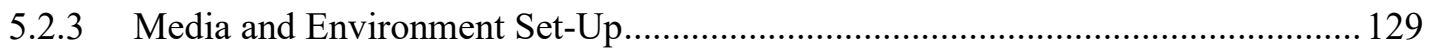

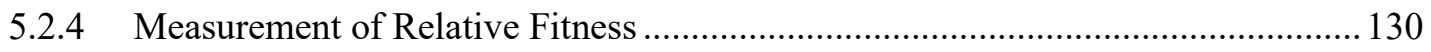

5.2.4.1 Nitrate-supplemented competitions ........................................................... 131

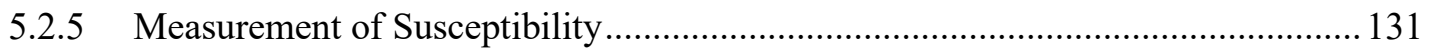

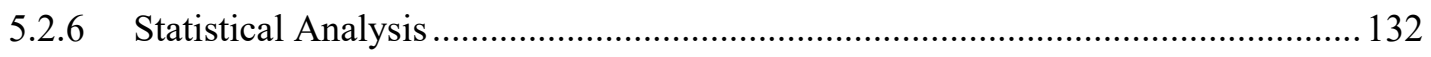

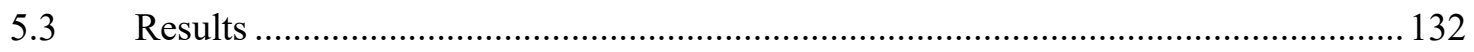

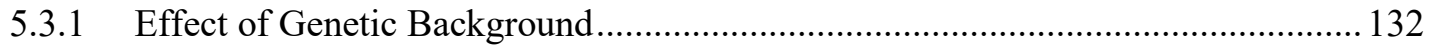

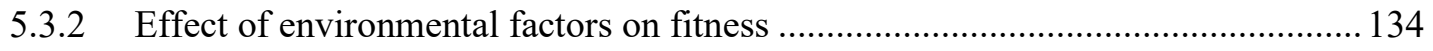

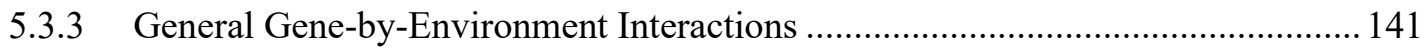

5.3.4 Effect of nitrate-supplementation on fitness in anoxic conditions ........................ 145

5.3.5 Impact of environment on drug resistance …............................................... 147 
5.4 Discussion.

Chapter 6: Conclusions and Future Directions..................................................... 163

Appendices ...................................................................................................................................... 170

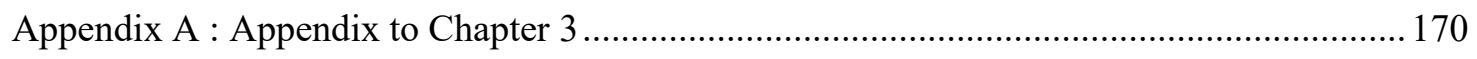

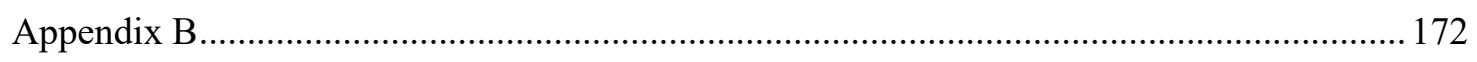

B.1 Sequencing and Parallel Evolution Supplementary Table ................................ 173

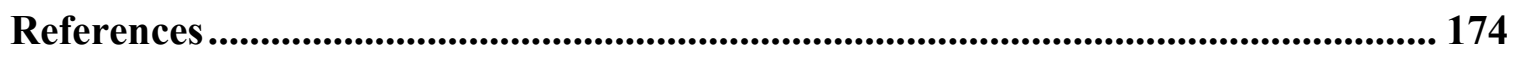




\section{List of Tables}

Table 2.1: Fitness changes associated with plasmid carriage......................................... 33

Table 2.2: Host-dependent fitness costs of plasmid carriage............................................. 35

Table 2.3: Persistence of plasmids during laboratory selection......................................... 44

Table 3.1: Serotype, infection source, and plasmid information for E. coli strains used in

this experiment.

Table 3.2: Plasmid prevalence after 100 generations. ........................................................ 71

Table 5.1: Serotype, source of isolate, and plasmid information for the E. coli and $K$.

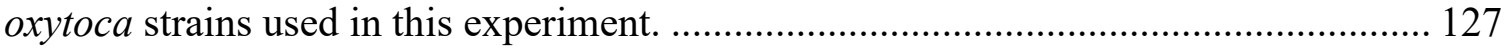

Table 5.2: Preparation of $50 \mathrm{ml}$ of $0.1 \mathrm{M}$ phosphate buffer to be mixed with $50 \mathrm{ml}$ of appropriate volume of 5X M9 salts and water............................................................ 129

Table 5.3: 3-way ANOVA for the effect of three environmental factors on fitness for MG1655

Table 5.4: 3-way ANOVA for the effect of three environmental factors on fitness for

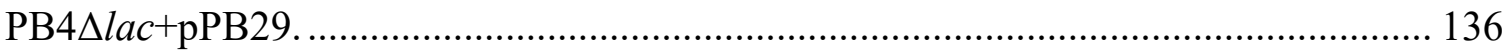

Table 5.5: 3-way ANOVA for the effect of three environmental factors on fitness for $\mathrm{PB} 4 \Delta l a c+\mathrm{pC} 33$.

Table 5.6: 3-way ANOVA for the effect of three environmental factors on fitness for $\mathrm{PB} 4 \Delta l a c+\mathrm{pC} 12$.

Table 5.7: Five-way ANOVA testing the impact of five factors (host, plasmid, oxygen presence/absence, glucose, and $\mathrm{pH}$ ) on fitness.

Table 5.8: 3-way ANOVA for the effect of three environmental factors on fitness for

$\mathrm{PB} 4 \Delta l a c+\mathrm{pC} 16$. 
Table 5.9: 3-way ANOVA for the effect of three environmental factors on fitness for MG1655 $l a c+$ pPB29. 


\section{List of Figures}

Figure 1.1: Types of epistasis relationships possible between two mutations, $a(\mathrm{w}=0.5)$ and $b(\mathrm{w}=0.75)$, when the fitness of both together is expected to be 0.375 (horizontal line).

Figure 2.1: Effect of RP4 on fitness of E. coli. Change in fitness (\%) for plasmid RP1 across E. coli strains 345-2RifC, 343-9, 99-24, 99-40, and K12 JM109, relative to the wild-type strain.

Figure 2.2: Selective sweeps of a plasmid to increasingly fit, ascendant strains

Figure 3.1: Effect of genetic background on the impact of plasmid carriage. 67

Figure 3.2: Conjugation efficiency of transconjugants.

Figure 3.3: Changes in fitness and resistance (minimum inhibitory concentration (MIC)) of three evolved transconjugants following 100 generations compared to the ancestral transconjugant.

Figure 3.4: Assessment of plasmid dynamics in mixed culture after 100 generations..... 74

Figure 4.1: The relative fitness in LB of three host-plasmid combinations following evolution with and without kanamycin selection for the plasmid over 500 generations.. 94 Figure 4.2: The relative fitness in LB of three host-plasmid combinations compared to the common competitor MG1655+pET28 following evolution with and without kanamycin selection for the plasmid over 500 generations.

Figure 4.3: The relative fitness in LB with $10 \mu \mathrm{g} / \mathrm{ml}$ kanamycin for three host-plasmid combinations compared to the common competitor MG1655+pET28 following evolution with and without kanamycin selection for the plasmid over 500 generations. 98 
Figure 4.4: The relative fitness in LB with $100 \mu \mathrm{g} / \mathrm{ml}$ kanamycin for three host-plasmid combinations compared to the common competitor MG1655+pET28 following evolution with and without kanamycin selection for the plasmid over 500 generations. 100

Figure 4.5: The number of mutations found for each clone in each genotype (MG1655, PB1, and OLC969), separated by the selection condition they were found in ( $\mathrm{Kn} 0.1$, Kn10, and LB). 104

Figure 4.6: The number of mutations per type (frameshift, intergenic, missense, stop, and synonymous) found in each of the genotype and selection condition. 105 Figure 4.7: Mutations observed in multiple selection conditions (LB, Kn0.1 or Kn10) and genotypes (MG1655, PB1, and OLC969) following 500 generations of experimental evolution. 108

Figure 4.8: Jaccard Index (J) for gene-level overlap between selection conditions for each genotype. 110

Figure 5.1: The fitness of six host-plasmid combinations averaged across twelve environments. 133

Figure 5.2: The average fitness of six host-plasmid combinations in media with $\mathrm{pH}$ 6.0, 7.0 , and 8.0 135

Figure 5.3: The average fitness of six host-plasmid combinations in media with low $(11.1 \mathrm{mM})$ or regular $(22.2 \mathrm{mM})$ glucose. 138

Figure 5.4: The average fitness of six host-plasmid combinations in the presence or absence of oxygen in modified M9+glucose media. 140 Figure 5.5: The relative fitness of six host-plasmid combinations in twelve environments. 
Figure 5.6: Relative fitness of MG1655 $l a c+\mathrm{pC} 12$ and $\mathrm{PB} 4 \Delta l a c+\mathrm{pC} 33$ grown in modified M9 medium with and without nitrate supplementation (10mM) both with and without oxygen. 147

Figure 5.7: Change in minimum inhibitory concentration (MIC) for six host-plasmid combinations measured in twelve environments.

Figure 5.8: Change in minimum inhibitory concentration (MIC) for four strains of E. coli bearing electron transport chain (ETC) mutations with plasmid pPB29, as measured in twelve environments. 152 Figure A.1: Fold change in MIC decrease, although not significantly, with starting MIC.

Figure A.2: Effect of genetic marker on recipient fitness. Effect of genetic marker ( $\Delta l a c$ or $\Delta l a c$-YFP) in MG1655, PB 1, 4, and 15, OLC682 and OLC969. 170 Figure A.3: Comparison of changes in resistance to changes in fitness. There is no correlation between the change in fitness and resistance 171 


\section{List of Appendices}

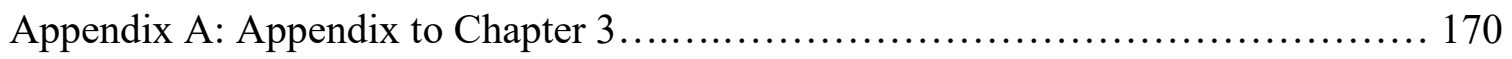

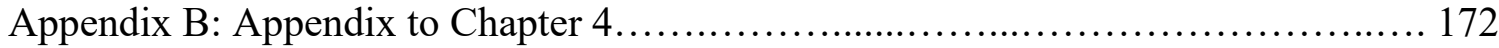




\section{Chapter 1: General Introduction}

Antimicrobial resistance (AMR) is currently one of the foremost threats to public health (O’Neill 2016). This threat, if left unchecked, has been predicted to result in 10 million deaths per year and cost the global economy $\$ 100$ billion by 2050 (O’Neill 2016). For example, there has been an increase of approximately five-fold in reported cases of colonization by carbapenemase-producing organisms (CPO) in Canada over the course of the last few years (Public Health Agency of Canada 2018). Since CPOs can be found in pathogens of concern (i.e., Escherichia coli and Klebsiella pneumoniae), and carbapenem antibiotics are some of the drugs of last resort, the rise in resistance to these drugs is concerning (Public Health Agency of Canada 2018; Academies 2019). Further, worldwide, the use of fluoroquinolone antibiotics to treat urinary tract infections caused by Escherichia coli is effective in less than half of patients treated (WHO 2018). Similarly, a recent review and meta-analysis looked at 39 studies which measured the resistance of $E$. coli strains isolated from human, animal, and food/environmental sources, and found that in humans, between 53 and $70 \%$ of the strains tested were resistant to amoxicillin (Pormohammad et al. 2019). Both examples show that there is a high prevalence of resistance to the more widely used drugs, as well as increasing resistance to drugs that are typically reserved for the treatment of more difficult infections. These examples clearly highlight the issues of AMR - left generally unchecked for any longer and these kinds of statistics will likely only get worse.

Despite the knowledge of this problem in the academic, medical, and public health spheres, there does not appear to be any one good solution to the problem. For example, drug discovery is a particularly enticing solution, in that the discovery of new, 
more effective drugs or drug classes might give an advantage in the fight against AMR (Simpkin et al. 2017). However, it is important to note that few new classes of antibiotic drugs have been discovered since the 1960s, so work has branched off into looking at other forms of compounds, such as antimicrobial peptides, to help reduce resistance or to bolster efficacy of current antibiotics (Coates et al. 2011; Travis et al. 2018).

Antimicrobial peptides are host-derived molecules which can be found in plants, animals, and microorganisms, and they have the ability to act similarly to antibiotic drugs to fight against bacteria and other microorganisms the host encounters (Aslam et al. 2018). Modifying existing antimicrobial peptides or creating novel ones, can be a way to combat AMR, though using these specifically as a treatment will require further research to make it a viable treatment option (Lei et al. 2019). Restriction protocols, whereby the use of certain drugs is limited to reduce resistance to them, have also been used to help extend the usefulness of currently known antibiotics. These approaches have been met with varying degrees of success, but the consensus is that they do have usefulness and work to some extent in many cases, particularly with reduced use in food-producing animals (Tang et al. 2017). Finally, alterations to the natural microbiota of humans, for example, through the use of fecal transplants or probiotics, have been proposed as a mechanism to displace resistant bacteria in that environment, thus altering the potential for the persistence of resistance or transfer of resistance to determinants to neighbouring bacteria (Relman and Lipsitch 2018).

In Through the Looking Glass by Lewis Carroll, the Red Queen explains to Alice: "Now, here, you see, it takes all the running you can do, to keep in the same place" (Carroll 1909). It has been suggested that the fight against AMR is "the Red Queen", as 
introduced by Leigh Van Valen in reference to this quote (Van Valen 1977). This concept argues that a species must constantly adapt, evolve, and reproduce to keep its footing while facing the evolution of competing species. In terms of AMR, bacteria have had to evolve to withstand the effects of antibiotics as they have been introduced to either kill competing bacteria in nature or to treat bacterial infections in animals and humans. For example, any time that there has been an introduction of a new compound that should kill bacteria, bacteria can quickly become resistant to it, and another compound may be introduced, which it then must evolve to withstand.

Of course, a great deal is understood about AMR: the major classes of resistance, how resistance occurs, rates of AMR mutations, the energetic cost associated with resistance, etc. However, there are still areas of AMR research where a lot is known, but not enough to combat the problem. One such area of research is plasmid biology. Certainly, a great deal is known about what plasmids are and what they can do (see below), but much is left to be uncovered. In particular, the "plasmid paradox" and how plasmid-mediated resistance can persist in the face of being costly, such that the fitness of plasmid-bearing bacteria is less than the plasmid-free ancestor, is still a major unknown (Harrison and Brockhurst 2012). Note, that if a plasmid-bearer grows more slowly and is unable to compete well against its plasmid-free ancestor, it will make up less of the population, and this will indicate a cost ("costly"). If the plasmid-bearing strain does better than the plasmid-free individuals, it will make up a larger proportion of the population, and this represents a fitness benefit ("more fit"). Here, I will address a few key areas of research that are currently less addressed in the literature, such as the long-term effect of selection on plasmid maintenance and parallel evolution, potential 
gene $\mathrm{x}$ environment interactions, and gene $\mathrm{x}$ gene interactions. Studying the impact of these factors should shed some light on how and why plasmids continue to persist as they do and aim to open new avenues of research which may further advance our knowledge in this field.

\subsection{Antibiotic Resistance}

Resistance to antimicrobials, a bacterium's ability to withstand antimicrobial drugs to which they may have been historically susceptible, is nothing new. In fact, resistance to these essential drugs has been found in microbial populations from before the widespread use of these compounds. Resistance is thought to have initially evolved as a mechanism to prevent antimicrobial producers from being adversely affected by drugs they themselves secreted (Peterson and Kaur, 2018).

Resistance to antibiotics can be inherent or acquired (Munita and Arias 2016). While some microbes can be inherently resistant to antimicrobial compounds, this can simply be the product of lacking structures that may be a target to the drug(s), such as those with different cell wall structures (Peterson and Kaur 2018). For example, vancomycin is ineffective against Gram-negative bacteria but combats Gram-positive bacteria because a lack of target availability. Vancomycin works by inhibiting cell wall synthesis, and because it is unable to cross the outer cell membrane in Gram-negative bacteria, it is unable to act on inhibiting cell wall synthesis; however, the cell wall of Gram-positive bacteria is accessible to the drug, as there is not outer membrane to pass, and the drug is able to exert its effects (Peterson and Kaur 2018). Acquired resistance, on 
the other hand, can be gained through mutation of target genes or the acquisition of mobile genetic elements (MGE) (Munita and Arias 2016; Peterson and Kaur 2018). It is important to note that the mechanisms of resistance that will be described here do not necessarily apply to any single drug class. Some general mechanisms, such as efflux, can confer resistance more broadly, while others, like target modification or drug breakdown, are more specific to the drug type and target, even if the overall mechanisms are similar. Examples of some of these resistance genes and mechanisms are listed in Table 1.1. Resistance to macrolide antibiotics, for instance, can occur through a variety of these same mechanisms, through modification of the target, drug inactivation, and efflux (Leclercq 2002). Sulfonamide resistance, on the other hand, is typically a result of the production of dihydropteroate synthetase (DHPS) which does not bind well to sulfonamide drugs (i.e. target modification) (Sköld 2000). Resistance to the drug classes aminoglycosides, $\beta$-lactams, and tetracyclines are more commonly found on plasmids, but are by no means the only AMR genes observed to be carried by these elements (Bennett 2008; Pal et al. 2015). The mechanisms of resistance for plasmid-encoded resistance genes are the same as those which are chromosomally encoded (Bennett 2008; Blair et al. 2015). 
Table 1: Genes associated with AMR to various antibiotic classes and their mode of action.

\begin{tabular}{|c|c|c|c|}
\hline $\begin{array}{l}\text { Resistance to Drug } \\
\text { Class }\end{array}$ & Mode of Action (Reygaert 2018) & Resistance Genes (Zhang et al. 2009) & $\begin{array}{l}\text { Type of Resistance (Reygaert } \\
\text { 2018) }\end{array}$ \\
\hline \multirow{2}{*}{$\beta$-lactam } & \multirow{2}{*}{ Inhibition of Cell Wall Synthesis } & $\begin{array}{c}\text { bla }_{\mathrm{TEM}}, \text { bla }_{\mathrm{SHV}}, \text { bla } \\
\text { (Freitas et al. 2019) }\end{array}$ & $\begin{array}{c}\text { Drug inactivation; cleavage of } \\
\beta \text {-lactam ring }\end{array}$ \\
\hline & & $o m p F$, ompC (Pagès et al. 2008) & $\begin{array}{l}\text { Prevent influx via reduction of } \\
\text { porins }\end{array}$ \\
\hline Aminoglycoside & $\begin{array}{l}\text { Inhibition of Protein Synthesis (30S } \\
\text { ribosomal subunit) }\end{array}$ & $\operatorname{aac}\left(6^{\prime}\right)-1 \mathrm{~b}, \operatorname{aac}(3)-\mathrm{IIa}$ & $\begin{array}{l}\text { Modification of drug; } \\
\text { acetylation, adenylation, } \\
\text { phosphorylation. Target } \\
\text { modification. }\end{array}$ \\
\hline Tetracycline & $\begin{array}{l}\text { Inhibition of Protein Synthesis (30S } \\
\text { ribosomal subunit) }\end{array}$ & tet $A$, tet $B$, tet $O$ & Modification of antibiotic \\
\hline Sulfonamide & Inhibition of Metabolic Pathways & sul1, sulII, sulIII, sulA, folP & Target Modification \\
\hline Quinolones & Inhibition of Nucleic Acid Synthesis & $\begin{array}{c}g y r A, g y r B \\
\operatorname{aac}\left(6^{\prime}\right)-\mathrm{Ib}-\mathrm{cr}(\text { Hooper and Jacoby } \\
2015)\end{array}$ & $\begin{array}{c}\text { Target Modification } \\
\text { Modification of Antibiotic } \\
\text { (acetylation) }\end{array}$ \\
\hline Macrolide & $\begin{array}{l}\text { Inhibition of Protein Synthesis (50S } \\
\text { ribosomal subunit) }\end{array}$ & erm $A$, ermB, ermC & Target Modification \\
\hline
\end{tabular}

*This list is by no means an exhaustive list of all possible acquired resistance genes. 
For example, aminoglycoside antibiotics are a class of bactericidal antibiotics that work by interfering with the protein synthesis of the target bacterium (Tenover 2006; Garneau-Tsodikova and Labby 2016). This is typically carried out by interactions with the $30 \mathrm{~S}$ ribosomal subunit, which prevents the accurate translation of protein from mRNA transcripts (Garneau-Tsodikova and Labby 2016). Inaccurate translation can lead to eventual protein misfolding, and this misfolding is thought to be a major early step by which aminoglycoside antibiotics exert their bactericidal effects (Goltermann et al. 2013). Resistance to this class of antibiotics is usually the result of one of three resistance mechanisms: alteration of ribosomal binding site, decreased cell permeability, and modification by enzymes (Garneau-Tsodikova and Labby 2016). Modification enzymes for aminoglycoside antibiotics can be encoded by genes such as $a a c\left(6^{\prime}\right)-1 \mathrm{~b}$ and $a a c(3)$ IIa (Zhang et al. 2009). These particular genes make aminoglycoside $N$ acetyltransferases, which will transfer acetyl groups to the aminoglycoside molecule to prevent its activity; the two examples above vary in the position which they acetylate the target, either at position 3 or 6 (Ramirez et al. 2013).

$\beta$-lactam antibiotics are also a class of bactericidal antibiotics, so named for their shared $\beta$-lactam ring structure, but this class works by inhibiting the biosynthesis of the cell wall rather than interfering with protein synthesis (Rice 2012; Peterson and Kaur 2018). The production of $\beta$-lactamase can confer resistance to this class of drug. This enzyme works by cleaving the $\beta$-lactam ring, which in turn inactivates the drug and prevents it from interfering with cell wall synthesis (Peterson and Kaur 2018). However, this is not the only way resistance to these drugs can occur; the production of altered penicillin-binding proteins in the bacterium can prevent binding of the drug without 
alteration of the drug itself (Rice 2012; Munita and Arias 2016). Resistance to these antibiotics can be conferred by a variety of genes, including bla $a_{\mathrm{TEM}}, b_{\mathrm{SHV}}, b_{\mathrm{CTX}-\mathrm{M}}$, and bla OXA (Zhang et al. 2009; Freitas et al. 2019).

Tetracyclines, so named for their four-ringed structures, are bacteriostatic antibiotics (Nguyen et al. 2014). Unlike the previous classes, they inhibit the growth of bacteria but do not actively kill them; in particular, these only impact actively growing cells (Nemeth et al. 2015). Resistance to these drugs can occur through several modes of action: drug efflux, ribosomal protection, and enzymatic inactivation, and this is encoded by the following mutations: tet $A$, tet $B$, and tet $O$ (Nguyen et al. 2014). Efflux of the drug by membrane proteins is most common, where drug that enters the cell will be pumped out (Nguyen et al. 2014). Next, ribosome protection proteins exist which prevent binding of tetracycline or which allow protein synthesis to occur despite conformational changes associated with drug binding (Nguyen et al. 2014; Munita and Arias 2016). Lastly, though less common, it is possible for tetracycline drugs to be inactivated by enzymes (Forsberg et al. 2015). 


\subsection{Plasmids and their persistence}

Bacterial plasmids are extrachromosomal DNA elements capable of transfer to other bacteria (Harrison and Brockhurst 2012). Transfer can be limited by host range of the plasmid or plasmid-donor or due to plasmid incompatibility, the inability of plasmids with the same replication system to be maintained in the same cell together, but otherwise may result in the rapid dissemination of the plasmid. Plasmids often carry multiple AMR genes, meaning that they are an attractive target for studies which aim to understand and reverse AMR (Pal et al. 2015). AMR genes are not the only kinds of resistance genes that can be plasmid-encoded, though, as genes which encode metal resistance/tolerance can also be found. For example, tolerance or resistance to mercury, arsenic, copper, and cadmium are frequently found on plasmids, and may be co-selected with AMR genes (Pal et al. 2015). Carriage of multiple determinants, be they resistance to antibiotics or tolerance to heavy metals, makes it increasingly likely for the plasmid to be selected by something, and improve its maintenance within populations. However, resistance and tolerance genes are by no means the only genes carried on these elements; indeed, plasmids will at least comprise of a basic replicon responsible for plasmid replication but will often have other genes that allow for more accurate plasmid partitioning, postsegregational killing, conjugative transfer, and more (Bennett 2008).

While one can see the benefit of resistance-bearing plasmids under exposure to antibiotics, they may not be cost-free to their host when that antibiotic exposure is removed. Plasmid-containing strains, for example, may be less capable of surviving and reproducing than their plasmid-free ancestor, and are thus considered 'costly'. By contrast, plasmid-carrying strains that are better able to survive and reproduce relative to 
their ancestors are more fit in the environment in which they reside. In general, it is thought that plasmid acquisition tends to be costly due to their metabolic burden on their hosts, though examples of cost-free and beneficial cases do exist (Enne et al. 2005; Cottell et al. 2012; Starikova et al. 2013; Vogwill and MacLean 2015).

Genetic interactions can be an important determinant of fitness and other phenotypes, such as levels of antibiotic resistance. That is, a mutation, or in this case a plasmid, can have one effect on fitness in one genetic background or host, whereas the same mutation or plasmid might have a completely different effect in a different genetic background. This is a phenomenon broadly known as epistasis, and can be characterized as positive, negative, sign epistasis, or reciprocal sign epistasis (Wong 2017). An example epistasis types can be found below (Figure 1.1). These cases can result when the fitness of a double mutant is different than what is expected from the single mutants alone, where the double mutant is more (positive) or less (negative) fit than expected. There is also the potential for sign epistasis, where the effect of a mutation varies depending on the genetic background of the host, such that the sign of one mutation changes, relative to the sign initially observed for the single mutant, once it follows another mutation (Ferretti et al. 2016; Wong 2017). Finally, reciprocal sign epistasis occurs when the two mutations together, be they both deleterious or beneficial, have the opposite effect than the two mutations alone (Wong 2017). 


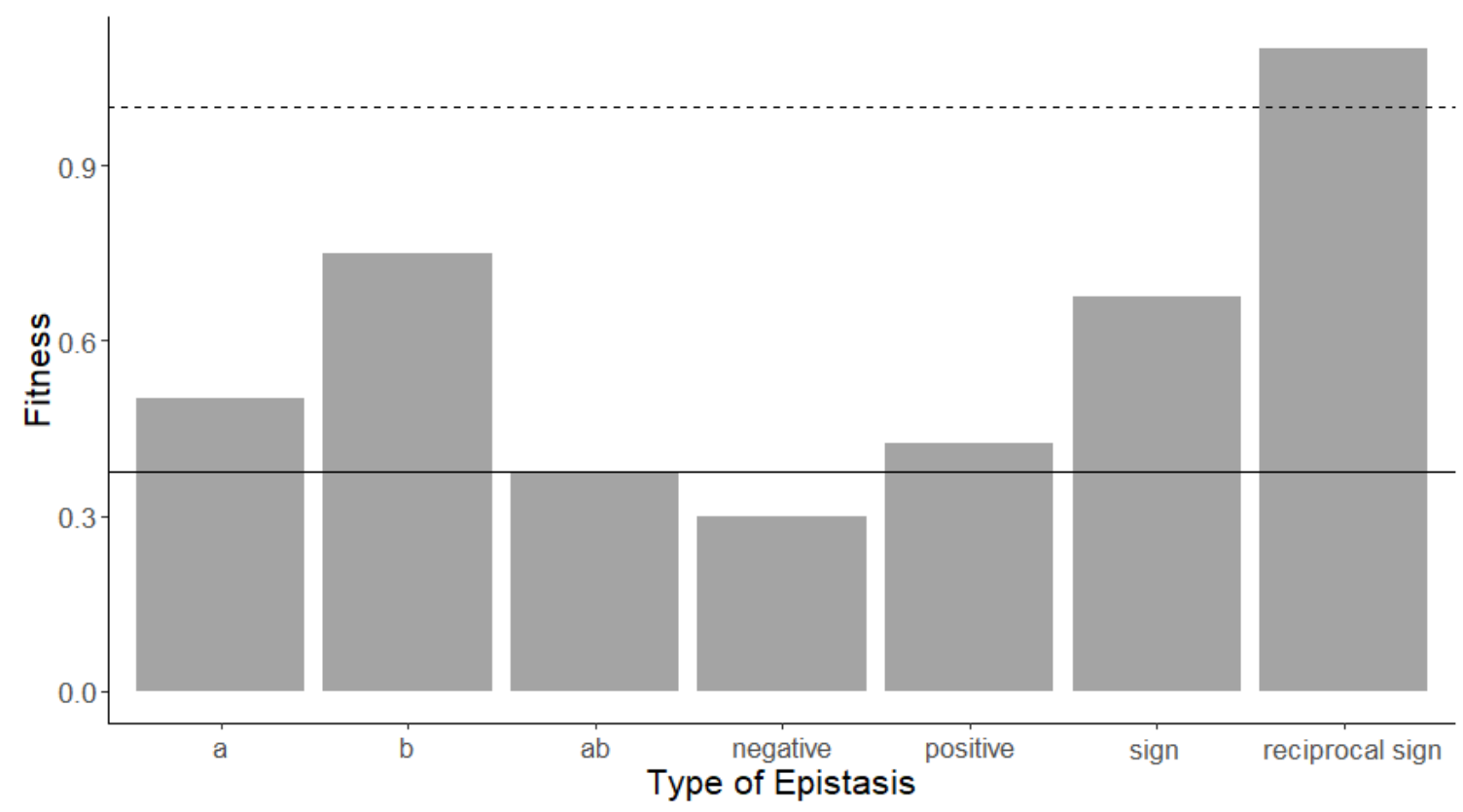

Figure 1.1: Types of epistasis relationships possible between two mutations, $a(\mathrm{w}=0.5)$ and $b(\mathrm{w}=0.75)$, when the fitness of both together is expected to be 0.375 (horizontal line). The combined fitness of $a$ and $b$ may be less (negative) or more (positive) than predicted by a multiplicative model, whereas the sign of one mutation may change depending on the presence of a previous mutation (sign) or the signs of both mutations might change (reciprocal sign). Fitness higher equal to 1 would indicate that the double mutant is more fit than the ancestral, mutation free background.

There is evidence of epistasis with host-plasmid combinations, as the genetic background of the host and the acquired plasmid may interact to produce different fitness outcome(s), so that the same plasmid might be costly in some genetic backgrounds, but might be beneficial or less costly in other backgrounds (Humphrey et al. 2012; Starikova et al. 2013; Di Luca et al. 2017). For example, Humphrey et al (2012) found that the plasmid RP1 had varying fitness costs in different strains of $E$. coli. Di Luca et al (2017) 
found that in E. coli and S. enterica, one plasmid (pG12-KPC-2) had varying costs, whereas another (pG06-VIM-1) did not vary between hosts. While a fair amount of the literature indicates that plasmid carriage is generally costly, some host-plasmid combinations can produce no cost relative to the wild-type or ancestral genotype, and some plasmids increase the fitness of the host (Enne et al. 2005; Cottell et al. 2012; Porse et al. 2016; Di Luca et al. 2017) . A meta-analysis by Vogwill and MacLean (2015) found that AMR plasmids were generally costly to their hosts, but not as costly as their chromosomal counterparts. While it is clear that epistasis plays some role in the fitness of a plasmid, some aspects are still unclear, and expanding on the types of plasmid, hosts, and types of analyses used to study these interactions will help to clarify certain aspects of plasmid biology.

A major question in the field of plasmid biology is that of plasmid persistence. The "plasmid paradox", a phrase coined by Harrison and Brockhurst (2012), has been used to describe how plasmids have managed to persist despite what challenges they might face and what evolutionary theory suggests (Harrison and Brockhurst 2012). That is, it is generally thought that plasmids will be lost over time whether they are costly or not, either because they are not necessary for survival and are lost, or because they are so useful that they become integrated into the host's chromosome (Bergstrom et al. 2000; Harrison and Brockhurst 2012; Carroll and Wong 2018). More specifically, a costly plasmid is expected to be lost due to purifying selection, such that we would expect total plasmid loss in a population following a long-term selection experiment. By contrast, a beneficial gene should theoretically integrate as a way to maintain its benefit, while reducing the burden of maintaining the rest of the plasmid in the cell (Bergstrom et al. 
2000; Harrison and Brockhurst 2012). Nonetheless, plasmids persist as plasmids for long periods of time, raising the question as to why. In Chapter 2 (published as Carroll and Wong 2018), I provide a systematic discussion of this issue.

Compensatory evolution can help to stabilize the presence of the plasmid while potentially reducing costs or other trade-offs that may otherwise have negatively affected the plasmid's ability to persist in its host (San Millan and MacLean 2017). There are many instances in the literature where costs are compensated to near wild type fitness following a selection experiment. For instance, Porse et al (2016) found that fitness improved following a period of coevolution from $6-12.5 \%$ decrease in growth rate to near wild type, and they report that this was due to the deletion of $\sim 25 \mathrm{~kb}$ of the plasmid. The genes lost from these deletions were involved in the main conjugation machinery, three resistance determinants, and a restriction-antirestriction system (Porse et al. 2016). Dahlberg and Chao (2003) found that there was up to a $70 \%$ increase in fitness of transconjugants following 1100 generations, both through changes to the plasmid and host, with decreased conjugation rates being observed following the selection period.

There is also evidence that conjugation can help maintain a plasmid in a population, even when costly (Lopatkin et al. 2017). That is, through both modelling and laboratory experiments, nine plasmids representing six incompatibility groups were able to persist via conjugation, as removing the ability of the plasmid to conjugate led to removal of the plasmid from the population over time. However, for conjugation to be a reliable method for maintenance, it is thought that conjugation rates may become so high that the plasmid will become parasitic (Hall et al. 2017a). In their review of the literature and use of modeling, Hall and colleagues highlight that lower plasmid conjugation rates 
are commonly found and that these rates are tightly controlled. This suggests that increased conjugation can become costly to the host, and that this process is highly regulated in this way to reduce the burden on the host when conjugation is not favourable and less likely to be successful. In these cases, increasing conjugation can be favourable when bacterial cell densities are high or conditions are otherwise ideal for successful transfer, but this is at best a good short-term way to maintain a plasmid; conjugation works best for shorter timescales, but host-plasmid coevolution becomes a more reliable long-term solution for plasmid persistence (Hall et al. 2017a). There is even evidence that there is a negative frequency dependent relationship with plasmid stability, such that the plasmid is stably maintained when at lower frequencies in the population (Dimitriu et al. 2019). As such, while conjugation might have a role to play in plasmid maintenance, there is conflicting evidence about the importance it plays in persistence on a broader scale.

Selection may also play an important role in plasmid persistence, such that continued exposure to antibiotics may make plasmid retention necessary, regardless of any cost that might be associated with it (Stalder et al. 2017). Yet, theory predicts that once the plasmid becomes beneficial, the plasmid is at risk of being lost through the integration of the gene(s) being selected, and the removal of the remaining part of the plasmid (Bergstrom et al. 2000; Harrison and Brockhurst 2012). One study by Modi et al (1992), found that there was integration of an ampicillin resistance gene, however, this was not a result of selection by ampicillin. Harrison et al (2015) found the integration of a transposon with a mercury resistance gene in response to mercury selection, however, these integrant clones did not frequently make up appreciable amounts of the population. 
Since loss through integration has not been widely reported in the literature, there are various hypotheses as to why this might be: compensatory evolution, conjugation, and selection. In fact, plasmids appear to be generally maintained over time across various conditions (ex. (De Gelder et al. 2007; Cottell et al. 2012; San Millan et al. 2014)). Selection can act to stabilize the plasmid so that compensatory evolution might have time to occur. Similarly, it can improve the fitness of the plasmid along the parasitismmutualism continuum, where the plasmid might be parasitic without selection, but could be mutualistic (i.e., beneficial) with increasing levels of selection (Harrison and Brockhurst 2012; Harrison et al. 2015). Selection of AMR plasmids over long periods of selection is not always required (for example, pKP33 in E. coli for 280 generations (Porse et al. 2016)), but is required for maintenance of pNUK73 in Pseudomonas aeruginosa PAO1 for 300 generations (San Millan et al. 2014b).

Notably, many laboratory studies of plasmid persistence are carried out for 300 generations or less. There are fewer studies observing persistence and changes in fitness on a longer scale without selection by antibiotics the plasmid encodes resistance for, such as work by Dahlberg and Chao (2003) who observed persistence for $\sim 1100$ generations, and one study observing the effect of one selection environment on fitness over 500 generations (Bouma and Lenski 1988). The outcome of long-term plasmid selection experiments with varying levels of selection does not appear to have been investigated beyond a single study where a host-plasmid combination was selected in varying concentrations of mercury for 450 generations (Harrison et al. 2015). However, they tracked only a single host-plasmid combination, which started out as very deleterious in the absence of antibiotic selection (Harrison et al. 2015). Note, that in terms of fitness, 
more costly host-plasmid combinations (with AMR gene(s)) will become less so as selection increases and as the resistance gene becomes more essential for survival, as the plasmid-free competitors, assuming they do not possess a chromosomal resistance gene, will be outcompeted.

The lack of these types of studies may be considered an oversight, as plasmidcontaining bacteria may not be exposed to antibiotics for such short lengths of time or might not always be exposed to lethal doses of the drug. Variation in both the length and level of exposure may have varying effects on plasmid persistence and fitness over time, particularly when the fitness of any plasmid could have variable fitness in its host and is therefore important to study. The impact that sub-inhibitory concentrations of antibiotic have on plasmid maintenance is of particular interest, since chromosomal AMR mutants can be selected for with as little as $1 / 1000^{\text {th }}$ the minimum inhibitory concentration (MIC) of some drugs (Gullberg et al. 2011). Recognizing that each of the three mechanisms discussed in depth above can act to stabilize a plasmid, the long-term effect of selection with or without drug when the plasmid has variable fitness in different genetic backgrounds should illuminate which mechanisms might be more likely to occur to maintain a plasmid within populations. I will address the impact of varied plasmid selection over 500 generations in Chapter 4, and I will attempt to identify the effect of host genetic background on this.

\subsection{Genotype $\mathbf{x}$ Environment Interactions in Plasmid Maintenance}

The importance of studies surveying the impact of various environments on bacteria with AMR mutations cannot be understated. However, many of the important 
studies which link AMR genes in whichever form they exist to being costly or beneficial are carried out in single or very few environments (Melnyk et al. 2015). Further, studies that have looked at AMR mutants in different environments have found variation in how those mutants will interact in the tested environments (Hall 2013a; Gifford et al. 2016). For example, Clarke et al found that the fitness of six AMR mutants of E. coli had marked gene-by-environment (GxE) interactions (Clarke et al. 2020). However, other studies have found that in Pseudomonas aeruginosa, four rifampicin resistant mutants retained similar patterns of fitness in various environments (Gifford et al, 2016).

Like GxG interactions, GxE interactions occur when a particular genotype has different fitness outcomes depending upon the environment it finds itself in (Hall 2013a). That is, a genotype broadly can be costly in one environment (such as in the laboratory in LB broth or some other rich growth medium) but could have no noticeable trade-off in fitness or even have increased fitness in another (soil, wastewater, etc.) and vice versa. These interactions would, as GxG interactions have shown, not allow for a simple conclusion in fitness outcomes of a genotype in alternate environments without fitness having been tested in those environments. Simply put, knowledge of fitness in one environment does not allow for the prediction of fitness in another. Indeed, a study by Kishony and Leibler (2003) suggested that deleterious mutations in E. coli could be rescued when grown in less optimal conditions, whereas other conditions would exacerbate deficiencies in growth for those strains, as was the case for acid stress (Kishony and Leibler 2003).

Intuitively, it may be evident that stressful environments can have negative impacts on species and strains of bacteria not well adapted to them. That is, the types of 
generally permissive environments used for laboratory studies, such as rich broths like Lysogeny Broth (LB) and others, may be mild for the organism(s) tested and an environment to which the organism(s) may already be well adapted to or that can be readily adapted to (Knöppel et al. 2018). Indeed, a recent article by Clarke et al (2020) showed that the fitness of several antibiotic-resistant mutants of $E$. coli varied significantly amongst multiple environments, concluding that measures taken in standard laboratory media may not accurately reflect actual fitness outcomes for strains studied in the lab across all environments.

While human antibiotic use certainly is a point of concern for contributing to AMR, these GxE interactions must also be considered, and thus the types of environments that could challenge them. It is understood that each species will have an optimal set of environmental conditions that are necessary for it to thrive. That is, there is an ideal temperature, $\mathrm{pH}$, level of oxygen, etc. that is necessary for the organism to function fully, though this is typically within a range of conditions that survival is certainly possible, but not optimal (Shehata and Marr 1971; Gadgil et al. 2005; CastaniéCornet et al. 2010; Von Wulffen et al. 2016). Beyond these boundaries of this range, the organism is not expected to survive. Further, there is evidence that lower quality environments, as measured by the yield of a strain within the environments tests, showed a positive trend with fitness, meaning fitness tended to be better in the lower yield environments (Clarke et al. 2020). This suggests that it is possible for a less-than-optimal environment to provide the right conditions for a plasmid-bearing strain to be more successful than its plasmid-free ancestor. 
The types of stressors that bacteria can encounter in various environments include changes in $\mathrm{pH}$, levels of oxygen, temperature, and nutrient levels, and these will be briefly summarized below. E. coli is a neutrophilic bacterium that prefers neutral $\mathrm{pH}$ levels. However, studies have shown that it is capable of growth in extremely acidic environments, with $\mathrm{pH}$ levels as low as 2.5 and below (Castanié-Cornet et al. 2010). Further, E. coli is a facultative aerobe, meaning that it can grow in the presence or absence of oxygen, however, it is noted that it is more energetically favourable to grow in the presence of oxygen (Von Wulffen et al. 2016). Growth for E. coli is optimal at $37^{\circ} \mathrm{C}$, though it is capable of growing above and below this optimum (Gadgil et al. 2005). Finally, varying levels of nutrients can also have an impact on overall growth and fitness of $E$. coli in different environments. For example, Shehata and Marr (1971) found that the growth rate of $E$. coli was independent of nutrient concentration when in excess, but dependent upon nutrient availability when levels were lower (Shehata and Marr 1971). However, bacteria are not necessarily just subject to these kinds of stressors; when infecting animals or humans, for example, bacteria may also be exposed to part of the host's innate immune system (Diamond et al. 2009). More specifically, part of this innate immune system includes antimicrobial peptides, which can have broad-spectrum activity against an invading pathogen (Diamond et al. 2009; Lei et al. 2019). This means that environment can have an increasing effect on measures such as fitness or resistance, such that these peptides can be effective against the pathogen in certain environments.

Environmental factors can also impact the degree of susceptibility that AMR bacteria can experience. There is evidence that aminoglycoside resistance, for example, will be increased in anoxic and low $\mathrm{pH}$ environments, whereas resistance to $\beta$-lactams 
shows the opposite trend, with resistance increasing with $\mathrm{pH}$ and in oxic environments (Schlessinger 1988; DeMars et al. 2016). While this study did not test these changes in resistance in animal or human models, they did test the resistance of isolates from cow feces, which can show the importance of environment on the resistance of bacteria which could, if food safety practices are lacking, infect humans who may consume these organisms. These trends are a result of mechanisms affecting the entry of the drug into the cell by impacting the electron transport chain, as well as by impacting the activity of the drug and its ability to interact with surface proteins of the cell for entry (Schlessinger 1988; Delcour 2009; Yang et al. 2014; DeMars et al. 2016). Further still, things like pH variation can impact the activation state of the drug, with increased or decreased $\mathrm{pH}$, and thus impact their ability to act on the cells (Blaser and Lüthy 1988; Atanasov et al. 2000; Lemaire et al. 2008; Delcour 2009). Taken together, the study of the effect that environment has on fitness and resistance, especially when factors are clearly defined and varied, is essential. This will not only show the importance of studying these interactions generally for AMR pathogens, but will also help pinpoint factors (such as glucoseavailability or $\mathrm{pH}$ ) which are particularly important for influencing these two measures.

\subsection{Parallel Evolution as a Tool for Understanding Host-Plasmid Co-Evolution}

Long-term evolution experiments, coupled with advances in whole-genome sequencing, have allowed microbiologists and evolutionary biologists to investigate the prevalence of parallel and convergent evolution in populations of bacteria. Parallel evolution occurs when a trait or some genetic change evolves independently in replicate populations of the same or closely related species (Futuyma et al. 1995; Schluter et al. 2004; Kassen 2014). This type of evolution is thought to be evidence of adaptation, since 
the same trait arising by chance in response to the same set of circumstances is thought to be unlikely (Bailey et al. 2015). In addition to selection, there are other factors which can lead to more frequent occurrences of certain mutations to certain genes (Bailey et al. 2015, 2017; Bailey and Bataillon 2016). For instance, the mutation rate across the genome can affect the likelihood of seeing mutations in some genes more often than in others, and any variation in selection for those mutations can impact which mutations are ultimately fixed in a population (Bailey and Bataillon 2016; Bailey et al. 2017).

Experiments investigating parallel evolution with plasmids are lacking; however, one study does show that parallel evolution to a global regulatory system occurs Pseudomonas fluorescens bearing pQBR103 across a variety of mercury selection levels, where the plasmid ranges from costly to beneficial in a single host (Harrison et al. 2015). This study appears to be one of very few studies on the prevalence of parallel evolution with respect to plasmids, and this certainly appears to be the only study utilizing varying selection conditions. Sota et al (2010) and Dimitriu et al (2020) found parallel evolution in plasmid replication genes as a response to selection. Sota et al found parallel evolution from selection in hosts where the plasmid is normally unstable, and Dimitriu et al found mutations which changed the rate of conjugation when plasmid-free immigrants are introduced to the plasmid-carrying population.

\subsection{Major Themes and Questions}

Plasmids have been studied since their discovery in the 1940s and much knowledge has been gained about what they do, how they are replicated, what types of genes they have, and how they might impact their hosts (Pinto et al. 2012; San Millan and 
MacLean 2017; Carroll and Wong 2018). However, there is still much to be learned about these mobile genetic elements, particularly in the face of the so-called 'plasmid paradox'. In my thesis, I aim to improve our understanding of the mechanisms of plasmid persistence, and the contribution of both $\mathrm{GxG}$ and GxE interactions on plasmid evolution.

\subsubsection{Chapter 3 Questions and Hypotheses}

The main questions in this chapter relate to the overarching patterns of epistasis that occur between the introduced plasmid and six E. coli hosts. More specifically, the questions I aimed to answer were as follows:

\section{Does host genetic background cause variation in the fitness effects and antibiotic resistance following the introduction of a single plasmid? Does starting fitness of the plasmid dictate how a plasmid will be maintained within a population?}

For the first question, I hypothesize that changes in fitness will be mediated by a genetic interaction between host and plasmid. That is, I expect that the fitness outcomes associated with a given plasmid will not be identical in each of the host genetic backgrounds. This expectation is based on previous, but limited, observations that fitness can vary depending on host genetic background (See Table 2.2).

For the second question, I predict that when a plasmid is costly, this cost will either be compensated to maintain the plasmid within the population, or the plasmid will be lost. As discussed above, the loss of initially costly plasmids is not necessarily observed as frequently as expected, as compensatory evolution, selection, or conjugation can act to stabilize the plasmid. I predict that compensation is the likely outcome for plasmid maintenance in initially costly individuals, rather than maintenance through 
conjugation or constant selection. This is because compensation is prevalent in the literature, and conjugation is still thought to be too costly to be the sole driver of plasmid maintenance.

\subsubsection{Chapter 4 Questions and Hypotheses}

Given that the impact of varied levels of antibiotic selection on plasmid-mediated resistance is not well studied, in chapter 4 I ask:

\section{What impact does varied levels of selection have on plasmid persistence, fitness, and parallel evolution?}

From this question, I can make several predictions. Firstly, I predict that populations evolved in with increased levels of plasmid selection will better maintain the plasmid than in populations where no selection was present. However, since the plasmid is expected to be less costly when selection for the plasmid is stronger, I predict that the types of mutations that might arise in the evolved populations will be different, as the need for compensation for any plasmid-borne costs might change. In terms of fitness, I expect that fitness will increase over the course of the selection experiment, but that the fitness of host-plasmid combinations evolved with increased levels of antibiotic selection for the plasmid will be more fit over time than the ones evolved in the absence of selection for the plasmid.

Evidence of parallel evolution might be less straightforward. I predict that I will observe parallel evolution in response to the same selection environment over time. However, I predict that parallel evolution will be more common in the host-plasmid combinations that began with a cost, and thus is further from a fitness optimum, as there 
would be more pressure for mutations to arise which help to alleviate costs when compared to the more neutral or beneficial host-plasmid combinations. Since I am using varied selection with varying levels of the drug $(0,0.1,10 \mu \mathrm{g} / \mathrm{ml}$ kanamycin), I predict that I will see variation in the types of mutations that arise in each of the selection conditions.

\subsubsection{Chapter 5 Questions and Hypotheses}

The main question to be addressed in this Chapter is this:

Are there gene-by-environment interactions for plasmids and do these extend to resistance?

I predict that there will be GxE interactions found for the host-plasmid combinations, and that I will see some of this effect not only in terms of fitness, but also with regards to resistance to the drugs that the plasmids carry resistance genes for. It is suggested that potential improvements in fitness in certain environments are a result of interactions which act to improve overall fitness when the right combination of genes and environment is met. Resistance may similarly react to changes in the environment. Experiments over the past few decades have investigated the impact of different conditions, namely the presence and absence of oxygen or varied $\mathrm{pH}$ and have found that drugs like aminoglycosides and $\beta$-lactams will have varying effectiveness depending on both of those conditions.

\subsubsection{Significance of Research}


I have highlighted areas which require further study to better understand plasmid fitness and persistence. Firstly, while there is evidence of plasmid fitness varying with genetic background, the long-term consequence of these interactions on the mechanisms responsible for plasmid persistence have not been studied extensively (addressed in Chapter 2 but studied in Chapters 3 and 4). Studying the long-term impact that fitness has on the strategies of plasmid maintenance will allow us to further understand why plasmids are able to continue existing beyond what theory might otherwise indicate. These results can help shape further studies that may identify situations where intervention might be useful. GxE interactions in plasmids, as studied in Chapter 5, on the other hand, have not, to the best of my knowledge, been presented in the literature. Since plasmids (and their hosts) can exist in many different types of environments, knowing if there are GxE interactions and identifying which environmental factors are important to fitness is essential. The results of this study may help us identify situations where fitness of a host-plasmid combination might be increased or decreased but could highlight ways to reduce plasmid persistence in those types of environments, and this can further have an impact on the treatment of bacterial infections. This study will also provide a foundation for further GxE studies with plasmids, which can be expanded to include different environmental factors, hosts, and plasmids. By expanding these kinds of studies, we can better understand both the spread and persistence of AMR plasmids in nature. Finally, in my long-term selection experiment, I expand upon the literature by investigating the effect of varied plasmid selection on fitness, persistence, and parallel evolution. Previous studies have investigated these effects but have failed to observe what happens when the same plasmid is present in different genotypes. We cannot be 
sure that the outcomes of fitness and parallel evolution following varied plasmid selection holds true for the same plasmid in more than the single genotype assayed and observing what happens between genotypes will expand our understanding of plasmid evolution and the factors which can influence it. Further, and perhaps most importantly, these results may lead to the design of effective novel treatments for AMR, which would have the potential to drastically improve outcomes for those with AMR infections.

\subsubsection{Applications}

In Canada, approximately $26 \%$ of bacterial infections were resistant to the drugs which would usually be used to first treat such infections in 2018, and left unchecked, this rate is expected to increase up to $40 \%$ by 2050 (The Council of the Canadian Academies 2019). At this rate, approximately 404 Canadians will die per year, as a result of an AMR infection (OECD 2018), and as many as 700,000 deaths per year were attributable to AMR infections worldwide (O’Neill 2016). Infections can occur in many body systems, including infections of the skin and soft tissue, blood, and the respiratory and urinary tracts, which highlights the need for studying the effect of environment on the fitness of bacterial pathogens in various environments (Jones et al. 2004). Further, approximately half of all natural bacterial isolates carry plasmids, meaning that understanding the biology of plasmids generally, and specifically AMR plasmids, will be essential to combatting the issue of AMR (Clark et al. 2018).

Plasmids frequently encode resistance to multiple drugs, thus making the treatment of infections which are caused by plasmid-carrying pathogens a major concern 
(Vogwill and MacLean 2015). While the purpose of this thesis was not to necessarily discover a way to combat these pathogens, insights gained through studying their biology during evolution experiments can shed light on what processes may be targeted to reduce AMR infections, as well as highlight if certain environments can be leveraged to reduce AMR pathogens prior to infecting a host. As discussed above, restriction protocols have shown some usefulness in reducing the prevalence of resistant pathogens (Tang et al. 2017). However, as my work shows that plasmid loss is not common and that costs can be reduced over time, the utility of such protocols may be minimal, and another approach would be necessary.

Ideally, if we can uncover the mechanisms which can increase the prevalence of the plasmid, novel antimicrobial treatments could be discovered to reduce those infections caused by plasmid-bearing E. coli. For example, since the conjugation of AMR plasmids can disseminate resistance within populations, as I show in Chapter 3, interfering with conjugation can be an attractive target for preventing resistance (Lopatkin et al. 2017). Similarly, if certain environments make AMR bacteria particularly vulnerable to treatment, or if certain points during the evolution of plasmid-host pairs similarly might favour the reduction of AMR, treatments might be designed to exploit these vulnerabilities and increase the tools which may be available to treat AMR infections.

More broadly, my findings might be applicable to studies in other systems. Hostplasmid interactions may mimic virus-host interactions, in that the plasmid or virus can "infect" its host, be it a bacterium in the case of the plasmid, or an animal or human for the virus. As such, the findings here may highlight broader evolutionary trends that could 
be applied to and used for viruses, such as changes to transfer rates or mechanisms to ensure survival in the host. 


\section{Chapter 2: Plasmid persistence: costs, benefits, and the plasmid}

\section{paradox}

\subsection{Introduction}

Bacterial plasmids are generally small (average $80 \mathrm{~kb}$ ) circular extrachromosomal DNA elements that are capable of semi-autonomous replication through the recruitment of host cell machinery (Sherratt 1974; Kado 2014; Shintani et al. 2015). Plasmids do not typically encode essential genes required by the host, but instead carry genes that may help the host adapt to novel environments, such as in the case of antibiotic exposure or alternative nutrient sources (Lili et al. 2007; Slater et al. 2008; Rankin et al. 2011). In addition, plasmids may carry genes encoding plasmid-specific functions, such as selfreplication, partitioning, and conjugative transfer (Bennett 2008). Despite the obvious benefit of plasmid-borne genes and traits under some circumstances, the reasons underlying continued plasmid persistence — as opposed to loss or integration — are incompletely understood; the puzzle posed by plasmid persistence has been coined "the plasmid paradox" by Harrison and Brockhurst (2012). Plasmid loss due to purifying selection would be expected when the costs of plasmid carriage outweigh the benefits. By contrast, integration would be expected for beneficial plasmid genes, minimizing random loss during cell division. In this review, we provide an overview of the benefits and costs of plasmid carriage and discuss the possible reasons for the continued persistence of plasmids. 


\subsection{Benefits of plasmid carriage}

The range of possible explanations for continued plasmid persistence has two extremes. At one end, plasmids exist entirely as selfish entities, only concerned in their continued survival (Bergstrom et al. 2000; Slater et al. 2008). At the other, plasmids can confer some unconditional benefit on their bacterial hosts. For the most part, neither extreme is likely to hold true - many plasmids are not unconditionally beneficial, insofar as they often confer costs on their hosts (see below). Similarly, transfer rates of most conjugative plasmids are likely not high enough for persistence as purely selfish elements, and of course nonconjugative plasmids are not readily transferred on their own (Bergstrom et al. 2000). Thus, we expect that most plasmids must be beneficial to their hosts - at least some of the time — to be maintained in populations.

Plasmid-encoded benefits are typically related to survival in novel environments and conditions, with many plasmids encoding genes for antibiotic resistance, virulence, tolerance to heavy metals, or for the metabolism of unique carbon sources, as well as nitrogen fixation, plant gall formation, and root nodulation (Masterson et al. 1982; Eberhard 1989; Nies 1999; Beceiro et al. 2013; Ramirez et al. 2014; Pal et al. 2015). Not only can plasmid encoded genes provide a potential benefit to their host, they may influence both the environment and neighbouring cells, for example, through production of helpful enzymes or nutrients or harmful chemicals (Rankin et al. 2011). Plasmids can thus be a source of genes that help the host adapt to novel environments (Eberhard 1989). Resistance to antibiotics is one of the better studied benefits conferred by plasmids, and one that is increasingly relevant in clinical settings. Plasmids carry resistance genes to most major classes of antibiotics, with genes for protection against aminoglycosides, $\beta$ - 
lactams, and tetracyclines most commonly found (Bennett 2008; Pal et al. 2015).

Plasmid-encoded resistance occurs via the same mechanisms as resistance caused by chromosomal mutations: by altering the permeability of the cell membrane, deactivation or modification of the antibiotic, and the modification of drug targets (Bennett 2008; Blair et al. 2015). Carbapenemases, for example, which include $\beta$-lactamases from classes $\mathrm{A}, \mathrm{B}$, and D, are now commonly found on plasmids, and these enzymes work by hydrolyzing and, thus, inactivating carbapenem antibiotics (Blair et al. 2015). Over and above issues with single resistance genes, multidrug-resistant (MDR) plasmids are increasingly common. For example, it was found that $40 \%$ of plasmids containing quinolone resistance genes that were isolated from strains obtained from dogs and cats with urinary tract infection confer MDR, with additional resistances to tetracycline, sulfonamides, or $\beta$-lactams frequently observed (Poirel et al. 2012; Liu et al. 2014). The rapid dissemination of MDR plasmids by horizontal gene transfer complicates both the treatment of infections and public health measures to control resistance.

\subsection{Deleterious effects of plasmid carriage}

While under particular conditions many plasmid-borne traits confer a benefit to their hosts, the wider fitness effects of plasmids are not always clear. For example, a plasmid may be beneficial when its bacterial host is exposed to a novel stressor, but detrimental once the stressor is removed (Vogwill and MacLean 2015). Under these conditions, it is expected that bacterial strains lacking the costly plasmid outcompete the plasmid bearing strain due to the fitness cost of plasmid carriage, and this cost may be a result of any one of a number of proposed mechanisms (Enne et al. 2004; San Millan and 
MacLean 2017). Thus, costly plasmids are expected to be lost from a population due to competition with plasmid-free cells. For instance, the non-transmissible plasmid pNUK73 in Pseudomonas aeruginosa is costly to its host compared with its plasmid-free ancestor and does not readily persist in the absence of selection (San Millan et al. 2014b).

Many studies have suggested that carriage of antimicrobial resistance (AMR) plasmids is generally costly to the host in the absence of antibiotic, although some evidence suggests this cost can be reduced over time (Dahlberg and Chao 2003; Dionisio et al. 2005b; Starikova et al. 2013; San Millan et al. 2014b; Johnson et al. 2015; Vogwill and MacLean 2015; Porse et al. 2016; Di Luca et al. 2017). This trend holds across plasmid types and bacterial species (Table 2.1), with fitness reductions ranging from $1 \%$ to $28 \%$. Non-AMR plasmids also often incur a cost (Hall et al. 2015). While plasmid carriage is typically costly, Vogwill and MacLean (2015) noted that the fitness costs associated with plasmids are generally less than the costs associated with chromosomal resistance mutations, suggesting that plasmid-borne resistance may sometimes be preferred to chromosomal resistance. However, plasmids by no means always confer fitness costs. For example, a pCT plasmid encoding an extended-spectrum- $\beta$-lactamase (ESBL) gene in Escherichia coli strains SW103 and J-53, as well as Salmonella enterica serovar Typhimurium SL1344, showed no measurable decrease in fitness, whereas a sulfonamide resistance-encoding plasmid p9123 increased the fitness of its E. coli host by $3.3 \% \pm 1.5 \%$ in the absence of selection (Enne et al. 2004; Cottell et al. 2012). 
Table 2.1: Fitness changes associated with plasmid carriage.

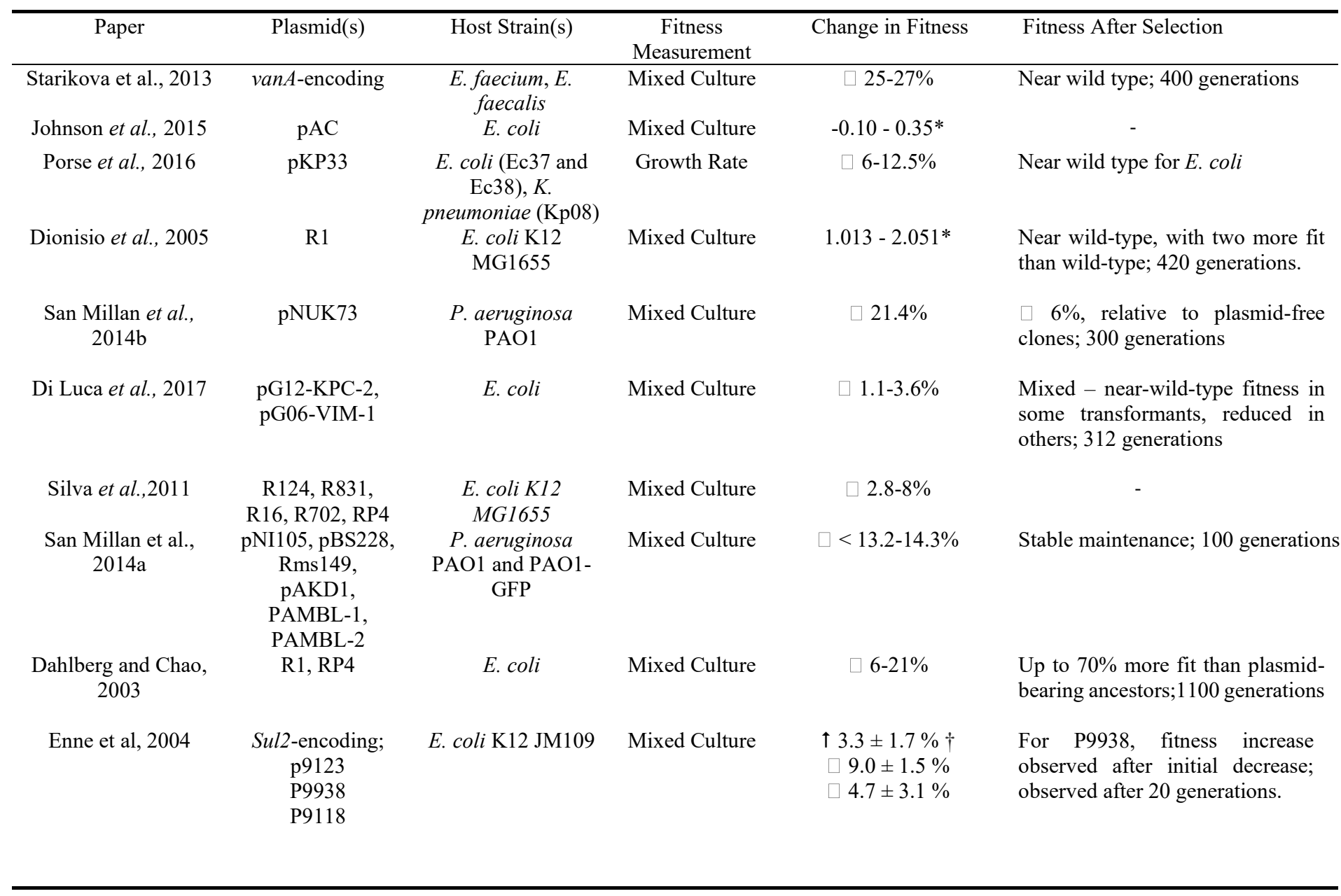

* Relative fitness

$\uparrow$ Indicates fitness increase 
Host genetic background may be an important determinant of plasmid fitness. That is, a single plasmid could decrease fitness in a strain with a certain genetic background, produce no noticeable fitness cost in another, and be beneficial in another strain (Table 2.2). For example, Humphrey et al. (2012) found that the plasmid RP1, when tested on five unique $E$. coli strains, had variable fitness effects, suggesting that the fitness cost of a plasmid is dependent on host genotype (Humphrey et al. 2012) (Figure 2.1). Indeed, Vogwill and MacLean (2015) indicate in a recent meta-analysis that both host genetic background and plasmid have some influence on the cost of carriage. Interestingly, such epistatic interactions do not appear to be limited to interactions between a plasmid and its host but can also include interactions between multiple plasmids in a single host strain (San Millan et al. 2014a). Here, two costly plasmids, a low-cost conjugative plasmid and a high-cost resistance plasmid, showed less severe fitness decreases when present together than were observed for each plasmid alone. 
Table 2.2: Host-dependent fitness costs of plasmid carriage.

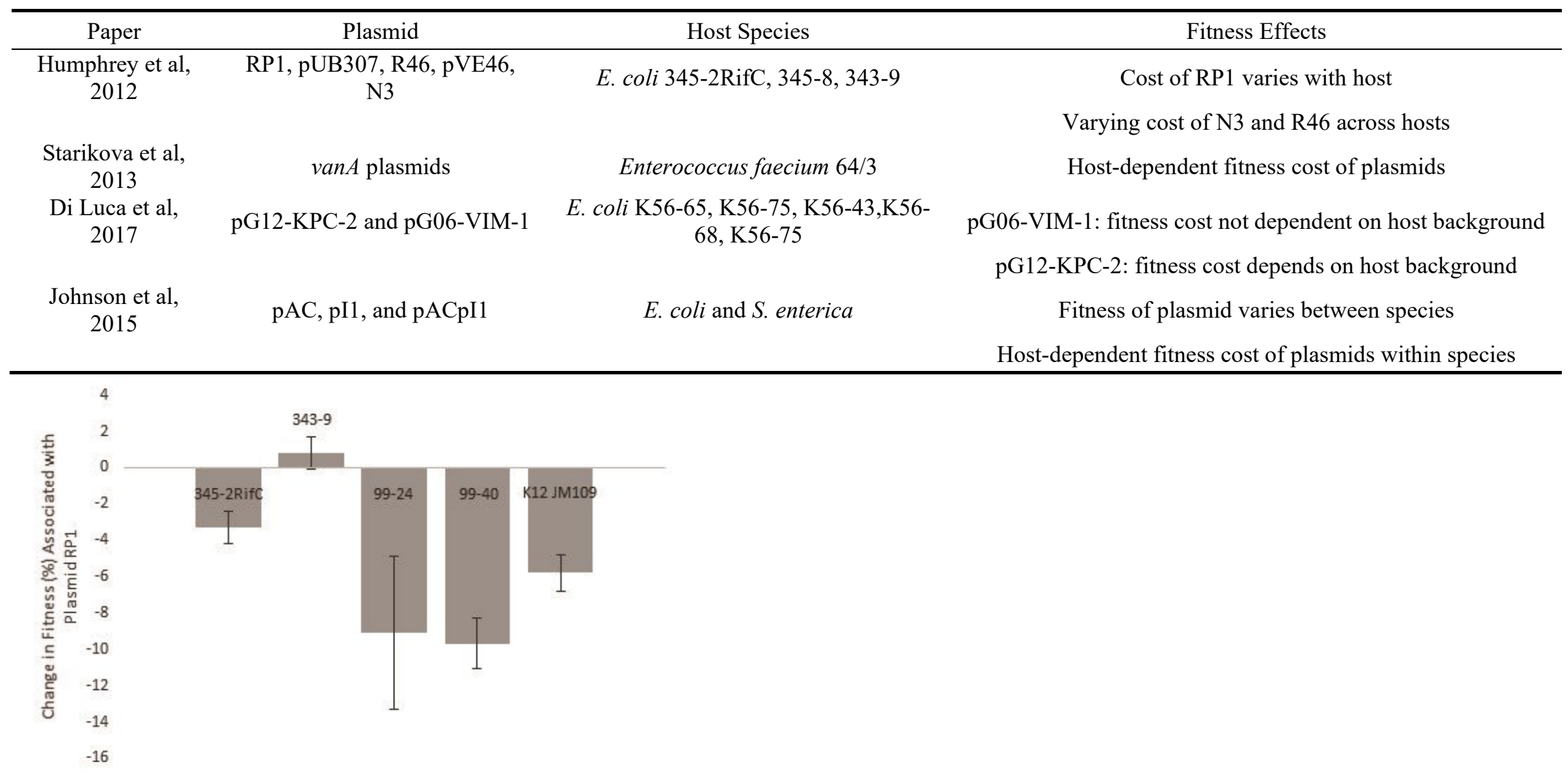

Figure 2.1: Effect of RP4 on fitness of E. coli. Change in fitness (\%) for plasmid RP1 across E. coli strains 345-2RifC, 343-9, 99-24, 99-40, and K12 JM109, relative to the wild-type strain. Figure produced by authors using data from Humphrey et al, 2012. 


\subsection{Mechanisms underlying the cost of plasmid carriage}

While the costs of plasmid carriage have been described widely, the mechanisms responsible for those costs are largely unclear. Several possible explanations have been proposed, ranging from the metabolic load introduced by plasmid replication and the expression of plasmid-borne genes to the disruption of essential host genes by the integration of plasmid genes, alteration in host gene expression, and other metabolic effects, such as the introduction of novel efflux pumps that may pump out important biomolecules (Pacheco et al. 2017; San Millan and MacLean 2017).

Here, a brief overview of each mechanism will be provided, but see San Millan and MacLean (2017) for an extensive review on the topic. The metabolic burden of plasmid carriage can come from the replication of plasmid genes. For example, replication of either the entire plasmid or specific plasmid genes diverts resources, such as biomolecules, machinery, or energy-rich compounds, away from the pool set aside for the replication of the host genome, which may stress the cell and reduce fitness (Baltrus 2013; San Millan and MacLean 2017). Consistent with this hypothesis, some studies have indicated that the deletion of large portions of a plasmid, which may also be associated with the loss of an antibiotic resistance marker, reduce fitness costs associated with those plasmids (Porse et al. 2016).

However, the expression of these same genes may also play a role in the resultant fitness cost associated with plasmid carriage and is thought to perhaps be the most likely candidate for plasmid-associated fitness costs (San Millan and MacLean 2017). Expression of plasmid borne genes can negatively impact fitness due to sequestration of ribosomes and issues stemming from codon usage and limited tRNA supplies. For 
example, a tetracycline resistance gene has been shown to induce fitness costs associated with the nonconjugative plasmid pBR322, and van genes have been identified as the main cause of fitness costs associated with some vancomycin resistance plasmids in Enterococcus (Lee and Edlin 1985; Valenzuela et al. 1996; Foucault et al. 2010). Similarly, it has been suggested that production of the conjugative machinery of the T4SS (type IV secretion system) system can contribute to fitness costs (Koraimann and Wagner 2014). Both the expression and the assembly of this system can induce stress responses in E. coli, which can in turn interfere with the host and impose a fitness cost (Zahrl et al. 2006). Nonetheless, it is important to note that nonconjugative plasmids, which lack transfer machinery, are also typically costly. Given that half of all plasmids are thought to be nontransmissible, but still are costly to their hosts, conjugation machinery cannot be the only contributor to fitness deficits (San Millan et al. 2014b).

Changes to the expression of host-encoded genes can also influence the fitness costs associated with plasmids. Indeed, there is good evidence that it is the misexpression of chromosomal genes that is responsible for fitness costs associated with pQBR103 in Pseudomonas fluorescens: about one fifth of chromosomal genes are mis-regulated following plasmid uptake, and compensation is accompanied by restoration of normal expression levels (Harrison et al. 2015). Here, mutations to gacA and (or) gacS, genes responsible for the global regulatory system, resulted in the decreased expression of about $17 \%$ of both plasmid and chromosomal genes. Inhibition of these genes by mutations to gacA and (or) gacS likely results in a decreased translational demand on the cell, reducing the cost of the plasmid, as the mutations result in the sustained inhibition of post-translational modification of RNA-binding proteins. 
Other more specific mechanisms have been proposed to explain the fitness costs associated with plasmid carriage. These include the integration of plasmids into the host chromosome, interference of plasmid genes and proteins with the host's normal functioning, as well as the efflux of important host proteins (Helling et al. 1981; Harrison and Brockhurst 2012; Hong et al. 2014; MacLean and San Millan 2015; San Millan et al. 2015). Integration can induce fitness costs as a result of the plasmid integrating into and interrupting important host genes (Jackson et al. 2011; Rankin et al. 2011; San Millan et al. 2015; Wu et al. 2015). Costs associated with plasmids may also result from the interference of plasmid-encoded elements with host structure and function; for example, plasmid-encoded molecules may interact negatively with the host's native proteins or be responsible for initiating processes like replication or protein expression, resulting in changes to host function and in fitness decreases (San Millan and MacLean 2017).

\subsection{Plasmid loss by segregational loss and integration}

Even in cases where plasmid carriage is not costly, plasmids are expected to slowly be lost from a population due to segregational loss, whereby a plasmid is lost by chance during cell division. Moreover, for plasmids carrying beneficial functions, a particular plasmid-borne gene can be integrated into the host chromosome and the rest of the element removed, or the entire element may be integrated, so that there is no segregational loss. Both of these processes can lead to population-wide plasmid loss over time.

Segregational instability is the term used to describe plasmid loss that occurs during cell division, whereby plasmids are not accurately segregated between daughter 
cells, resulting in plasmid-free individuals (Popov et al. 2009). This is of concern for lowcopy number plasmids, where fewer copies of the plasmids are present in the host. Since this loss may be problematic to the continued survival of the plasmid, specific mechanisms have evolved to ensure accurate plasmid distribution between cells, such as parI and parII, which are part of the active partitioning systems for some plasmids (Million-Weaver and Camps 2014). However, if these systems are inefficient and segregational loss does occur frequently enough, even in the face of such mechanisms, and this rate is higher than that of horizontal transfer, plasmid loss within the population is the expected outcome in the absence of plasmid selection (Hernández-Arriaga et al. 2014; San Millan et al. 2015). Estimates of the rate of segregational loss vary widely. For example, a segregational loss rate of $10^{-3}$ per cell per generation was estimated for an R1 plasmid derivative, which was much less frequent than a previous estimate of $1 \%-5 \%$ per cell per generation found in other studies (Lau et al. 2013). Higher rates were estimated for the pACY 184 plasmid in E. coli B, at approximately 0.02 per cell per generation without selection, and 0.01 per cell per generation with a certain level of selection (Lenski and Bouma 1987).

Given that plasmids are at risk for loss over time, mechanisms have evolved that reduce the frequency of plasmid-free bacteria in a population. One such example is that of the post-segregational killing (PSK) system, which kills individual cells that lack the plasmid (Hernández-Arriaga et al. 2014). This is especially important for plasmids maintained at low-copy numbers (Dmowski and Jagura-Burdzy 2013). PSK, which can also be referred to as the toxin-antitoxin (TA) system, works by either removing cells without plasmids or by increasing plasmid replication to ensure copy number 
maintenance (Hayes and Van Melderen 2011; Dmowski and Jagura-Burdzy 2013). These systems are typically arranged so that there is a chromosomally encoded toxin that is constantly produced by the cell under normal conditions, and the plasmid-encoded antitoxin is also expressed, interfering with or inactivating the toxin (Brendler et al. 2004; Hernández-Arriaga et al. 2014). However, if the plasmid is accidentally lost during a cell division event, that antitoxin is no longer produced, whereas the toxin is still expressed, which then can either severely inhibit growth of, or kill, the plasmid-free host. Nevertheless, plasmid loss is still observed in systems with an active PSK system (San Millan et al. 2014b). Additionally, observations of TA and PSK showed that plasmid levels remained steady regardless of whether the TA genes were encoded on the plasmid or on the host chromosome, suggesting that the TA system may have evolved to prevent colonization of other plasmids rather than to intrinsically improve plasmid stability (Hernández-Arriaga et al. 2014). Moreover, as Harrison and Brockhurst (2012) note, there are examples of plasmids persisting in the absence of these TA systems, so these systems must not be the sole reason for plasmids remaining in their hosts.

The importance of segregational loss may be in part determined by plasmid size and copy number. Small high-copy number plasmids (up to $100 \mathrm{~kb}$ in size, and $>10$ copies/cell) are generally thought to be randomly distributed between daughter cells during division, as they lack adequate partitioning and PSK mechanisms, though there is some evidence of plasmid localization at the poles of dividing cells to aid in partitioning (Million-Weaver and Camps 2014; Millan et al. 2016; Wang 2017). Because of their higher copy numbers, chances are low that either daughter cell will be plasmid-free. This mode of plasmid partitioning may facilitate adaptive evolution, as the genes on these 
plasmids also exist in high copy number in the cell. This increases the chance of gaining beneficial mutations and depending on the number of copies divided among the cells, selection may act more or less efficiently on the mutated copies (Millan et al. 2016).

Unlike small, high-copy number plasmids, larger, lower-copy number plasmids require specific mechanisms to ensure propagation of the plasmid, such as the tight regulation of replication and copy number control, and frequently encode toxin-antitoxin systems (Carattoli 2013; Tsang 2017). Carrying beneficial genes, such as antibiotic resistance genes, can provide a basis of plasmid selection, increasing maintenance of the plasmid and making it more likely that the plasmid may coevolve with the host over time (Stalder et al. 2017). The presence of these sometimes-beneficial genes increases the survivability of plasmids because they may be more readily expressed on a plasmid than on the chromosome, and having these benefits can increase the niche range of the host, mitigating fitness costs (Eberhard 1990; San Millan et al. 2014b). Beyond this, however, strategies employed by the plasmid can increase persistence. For example, large conjugative resistance plasmids are thought to have three distinct coevolutionary patterns that act to increase persistence, which include mutations to replication genes, mutations to the global transcriptional regulatory system of the host, as well as the acquisition of a toxin-antitoxin encoding transposon from another plasmid with an established system (Porse et al. 2016). Porse et al. (2016), for example, found that persistence was accompanied by changes in the plasmid backbone (i.e., deletions of costly portions of the plasmid), the acquisition of a toxin-antitoxin transposon, and changes to the pattern of transferability, to improve the vertical, rather than horizontal, inheritance of the element. 
Nontransmissible plasmids, as they generally lack the ability to persist through high transfer rates, are thought to increase stability through the compensation of plasmidassociated fitness costs and plasmid selection (San Millan et al. 2014b). For example, positive selection of the plasmid pNUK73, a costly nontransmissible plasmid in $P$. aeruginosa, is required to offset the potential loss of plasmid by segregational loss (San Millan et al. 2014b). However, there is some evidence that nontransmissible plasmids may very rarely accomplish horizontal gene transfer, which can help stabilize the plasmid in a population (Peña-Miller et al. 2015).

\subsection{The plasmid paradox}

We expect that plasmids, whether costly or beneficial to their hosts, to either be lost over time or integrated into the host chromosome, but we do not expect that they will persist. Yet, there is much evidence indicating that plasmids, whether costly or not costly, selected for or not, can continue to persist over long periods of time (Table 2.3; Harrison and Brockhurst 2012; MacLean and San Millan 2015). This long-term persistence has been coined the "plasmid paradox" (Harrison and Brockhurst 2012). In laboratory studies, where plasmid persistence is typically measured through serial transfers of the strain of interest in nonselective media over time, plasmids are often retained. While plasmid persistence is often observed, the level of selection required by the plasmid to persist varies, from no selection whatsoever to varying levels of antibiotic selection (Bergstrom et al. 2000; De Gelder et al. 2007; Heuer et al. 2007; Fischer et al. 2014; San Millan et al. 2014b). For example, the ESBL-encoding plasmid pCT persisted in E. coli for approximately 70 generations in the absence of any antibiotic selection (Cottell et al. 
2012). Consistent with the lack of requirement for selection, the removal of a resistance gene from $\mathrm{pCT}$ did not change the level of plasmid persistence at the end of a selection experiment (Cottell et al. 2012). Similarly, a MDR plasmid in E. coli was relatively stable over time, regardless of selection for that plasmid using antibiotics (Porse et al. 2016). However, other studies have indicated that varying levels of antibiotic selection are required for persistence, for example, antibiotic selection was needed for the maintenance of the nontransmissible plasmid pNUK73 in P. aeruginosa PAO1 (San Millan et al. 2014b; Johnson et al. 2015; Löhr et al. 2015). Over deeper evolutionary time, plasmids have persisted in louse symbionts for upwards of 35 million years (Boyd et al. 2017). In this case, it appears that essential genes encoding for vitamin B5 synthesis have moved to the plasmid, suggesting that this configuration may have some benefit for both the plasmid and organism (Boyd et al. 2017). 
Table 2.3: Persistence of plasmids during laboratory selection.

\begin{tabular}{|c|c|c|c|c|}
\hline Publication & Selection Conditions & Plasmid & Host Organism & $\begin{array}{c}\text { Duration of } \\
\text { persistence } \\
\text { (generations) }\end{array}$ \\
\hline San Millan et al, 2014b & Antibiotics required & pNUK73 & P. aeruginosa $\mathrm{PAO} 1$ & 300 \\
\hline Cottell et al, 2012 & Persists without selection & $\mathrm{pCT}$ and $\mathrm{pCT} 2$ & E. coli & 70 \\
\hline Fischer et al, 2014 & Persists without selection & IncI1 family & E. coli & $385-651$ \\
\hline De Gelder et al, 2007 & Variable; depends on host & pB10 & $\begin{array}{c}\text { Alpha-, Beta-, and } \\
\text { Gammaproteobacteria }\end{array}$ & 200 \\
\hline Porse et al, 2016 & $\begin{array}{l}\text { Persists without selection; } \\
\text { coevolution hypothesized }\end{array}$ & pKP33 & $\begin{array}{l}\text { E. coli } \mathrm{Ec} 37, \mathrm{Ec} 38, K . \\
\text { pneumoniae Kp08 }\end{array}$ & 280 \\
\hline
\end{tabular}


At least part of the plasmid paradox can be resolved by host-plasmid coevolution (Harrison and Brockhurst 2012; MacLean and San Millan 2015). The costs of plasmid carriage can be ameliorated by mutations to either the plasmid or the host; the accumulation of such mutations over time is referred to as compensatory evolution (Harrison and Brockhurst 2012). We note that the genetic background effects referred to above differ from compensatory evolution only in the timing by which compensatory mutations arise — prior to plasmid acquisition in the case of background effects, and afterwards in the case of compensatory evolution (Wong 2017). Compensatory evolution is easily achievable in the laboratory; studies tracking changes in fitness over time have generally found that under nonselective conditions, plasmids can remain in the population and the plasmid-related fitness costs may be reduced, returning to almost wild-type fitness (San Millan et al. 2014b; Porse et al. 2016). For example, Yano et al. (Yano et al. 2016) showed that plasmid persistence in the absence of selection may be a direct result of host-plasmid coadaptation. Here, mutations to TrfA1, a protein for replication initiation of the plasmid pMS0506, were associated with increased persistence. Additionally, compensatory evolution can occur quite rapidly when the mega-plasmid pQBR103 is transferred to $P$. fluorescens, rescuing the host from previously deleterious effects associated with this plasmid. Compensation occurs via mutations to the global regulatory genes gacA and gacS (Harrison et al. 2015). Importantly, the presence of compensatory mutations not only ameliorates the fitness cost of the plasmid, thus allowing it to be maintained even in the absence of selection, but may also provide a means by which plasmid loss would be detrimental to the cell (Schrag et al. 1997; Wong 2017). That is, the compensatory mutation(s) may only be beneficial so long as the 
element for which it compensates is present and are deleterious if the plasmid is lost. The fitness effects of compensatory mutations in the absence of plasmids, however, have not been thoroughly investigated.

Fewer studies have investigated the mechanisms and mutations contributing to compensation, but in one study a $25 \mathrm{~kb}$ deletion on a plasmid was typically found in strains that had reduced fitness costs but still maintained the plasmid (Porse et al. 2016). In the case of Dahlberg and Chao (2003), the absence of resistance markers was found occasionally after periods of coevolution, suggesting loss of the gene may be behind the reduced fitness cost for carriage of that plasmid in some individuals. Conversely, San Millan et al. (2014b) did not find any plasmid mutations from six clones containing a plasmid, but instead found single compensatory chromosomal mutations to either a helicase or a protein kinase gene. These results concur with work done by Helling et al. (1981) and Bouma and Lenski (1988), which demonstrated that compensatory mutations were located on the host chromosome rather than on the plasmid.

While host-plasmid coevolution helps to explain the persistence of plasmids in the face of costs and of segregational loss, it is worth emphasizing that beneficial plasmidencoded genes that may be on an otherwise costly plasmid may integrate into the host chromosome, creating chromosomal variants with the gene of interest. Nevertheless, host chromosome integration is rare in the laboratory (Table 2.3), and at least some plasmids persist as independent entities for millions of years (Boyd et al. 2017). The occurrence of coadaptation does not adequately explain why integration is an exception, rather than the rule, since strong selection should favour integration of beneficial genes (Bergstrom et al. 2000). 
There is some evidence for selection against integrants, which may help to explain some cases of long-term plasmid persistence. While integration is possible, it is frequently unstable in the instances when it does occur, and there is evidence that cells prefer the presence of separate replicons for each element, rather than a larger integrated one (Mavingui et al. 2002; Heap et al. 2012). The instability of integration, beyond the potential introduction of fitness costs, may be a result of disrupting the symmetry between the origin and termini of replication ( $\mathrm{oriC}$ and TER sites). This asymmetry may interfere with cellular activity and cause the cell to favour excision of the inserted DNA or to introduce inversions to restore balance (Alokam et al. 2002). For example, the insertion of TN10 into $r r n H / G$ and proB of S. enterica serovar Paratyphi A resulted in an inversion to compensate for the asymmetry between oriC and TER. However, it has been noted that inversions in certain locations in the genome are deleterious, and that inversions and other rearrangements may not survive in wildtype strains as they do in culture (Liu and Sanderson 1995; Alokam et al. 2002).

Alternative explanations for the persistence of plasmids as independent replicons argue that a plasmid's ability to move is precisely what keeps it from being integrated, and thus lost as an independent element. Under one model, plasmids "hitchhike" on increasingly fit genetic backgrounds, which allows them to outpace the fitter strains with chromosomally encoded plasmid genes (such as an antibiotic resistance gene, for example). This occurs in a cyclical manner, as described by Bergstrom et al. (2000). It is assumed that there is a focal gene, for example, an antibiotic resistance gene that can either be encoded by the plasmid or be integrated into the host chromosome; this may create chromosomal variants with the initially plasmid-encoded gene. This allows the 
host to retain the advantageous gene and it may gain a selective advantage, as it lacks the costly element itself, which is then at risk for loss in the population. If a new strain arrives within the area that has some novel beneficial characteristic, and it begins to outcompete the other strains, the plasmid at risk for loss can transfer to this strain as it sweeps through the population. The beneficial plasmid gene then has a potential to integrate into the chromosome, beginning the cycle again, where the plasmid faces removal from the population unless it can hitchhike along with another ascendant strain. A graphical representation based on this process described by Bergstrom et al. (2000) shows the changes in fitness for different variants carrying a focal gene originally found on a plasmid over time (Figure 2.2). However, while this model is attractive given that plasmid persistence appears to be common, it is only valid so long as the most fit, ascendant strain does not already encode the beneficial gene that may have been found on the plasmid in the first place. 


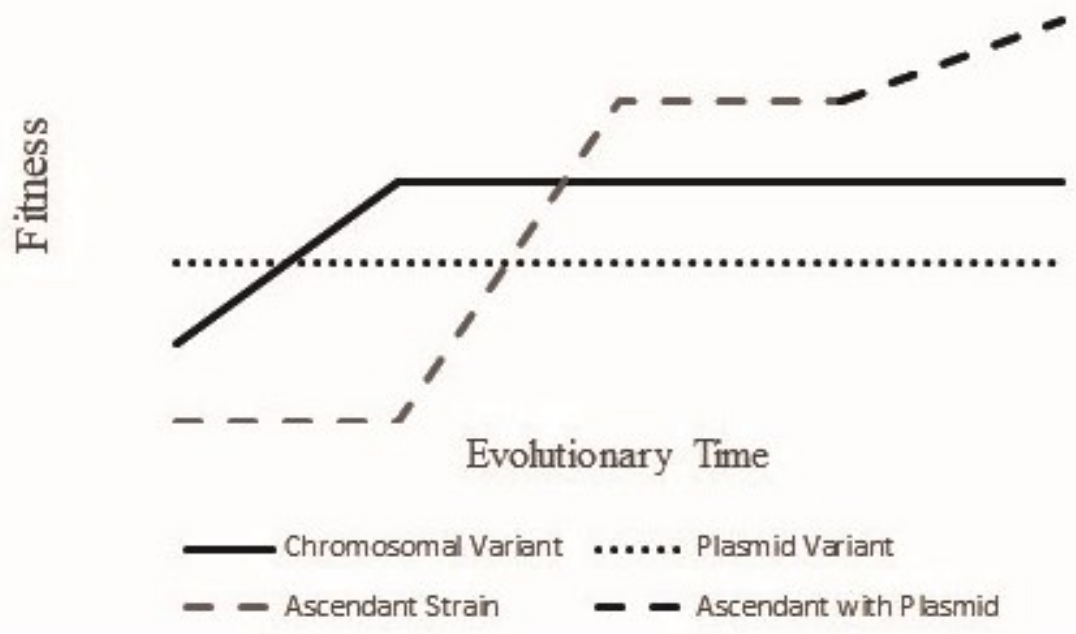

Figure 2.2: Selective sweeps of a plasmid to increasingly fit, ascendant strains. Initially a strain with a chromosomal variant of a focal plasmid gene (solid black line) becomes more fit than a strain with a plasmid-encoded gene (dotted black line). The ascendant strain (dashed grey line), which contains novel beneficial characteristics, enters the population with increased fitness over both the strains carrying either the plasmid or chromosomally-encoded focal gene. The plasmid can transfer to this ascendant strain, potentially increasing fitness (black dashed line), which has increased fitness relative to the original ascendant strain, and the process may begin again. Figure produced by authors using information from Bergstrom et al (2000).

The cross-ecotype model attempts to explain how plasmids persist beyond purifying events, such as a selective sweep (Bergstrom et al. 2000). Briefly, an ecotype is a distinct population inhabiting a given environment, which presumably diverged from the original population due to ecological speciation (Koeppel et al. 2013). Using again an example of a focal antibiotic resistance gene that can either be chromosomally or plasmid encoded, it can be assumed that chromosomal variants are preferred to the same gene on a plasmid. However, the chromosomal variant may be lost over evolutionary time due to 
mutation or deletion events (Bergstrom et al. 2000). While overall the chromosomal variant is preferred and will outcompete genotypes containing the plasmid-encoded variant, they are unable to transfer across ecotypes, unlike their plasmid-encoded counterparts. As such, recolonization of the plasmid and the focal gene is possible, even between ecotypes, if one assumes that the fitter chromosomal variant has been lost by chance over time. If, for example, that chromosomal variant is still present in the population, it is unlikely that the plasmid-encoded variant will successfully recolonize the population if it is still costly to maintain. This model thus provides one explanation for why certain types of genes, like an antibiotic resistance gene, are commonly found on plasmids - they are unlikely to be lost completely due to the ability to transfer across ecotypes, when compared with chromosomally encoded variants. Similarly, it has been argued that plasmids may exist as local adaptations, and that their ability to be readily transferred between bacteria can allow for the recipient to adapt to the environment if the plasmid provides a benefit in those conditions (Eberhard 1989).

The presence of high plasmid transfer rates has also been proposed as a possible solution to the plasmid paradox. Models suggest that plasmid persistence is likely so long as the plasmid is occasionally beneficial and horizontal transfer rates are sufficiently high to overcome plasmid fitness costs and segregational loss (Svara and Rankin 2011). In these models, when the rate of horizontal transfer for a plasmid is low (transfer rate $\beta=$ 0.01 cells $\cdot \mathrm{mL}-1 \cdot \min -1)$, chromosomal resistance genes are favoured under short and strong selection, whereas when rates are higher $\left(\beta=0.1\right.$ cells $\left.\cdot \mathrm{mL}^{-1} \cdot \mathrm{min}^{-1}\right)$, plasmid encoded resistance genes will be favoured (Svara and Rankin 2011). Notably, however, they do not consider integration as a possibility for plasmid loss (Slater et al. 2008). 
Gordon (1992) and Wan et al. (2011) have estimated that the plasmid transfer rate in nature is between $1.3 \times 10^{-15}$ and $3.0 \times 10^{-8}$ cells $\cdot \mathrm{mL}^{-1} \cdot \mathrm{h}^{-1}$, based on experimental data with various laboratory and environmental E. coli strains, but the exact rate is unknown. Moreover, transfer rates may be higher in biofilms than in planktonic cells (Ghigo 2001; Stalder and Top 2016). More broadly, high transfer rates do not provide a good explanation for the persistence of nonconjugative and nonmobilizable plasmids, as these plasmids are unable to transfer on their own (Harrison and Brockhurst 2012). Moreover, Hall et al. (2017) suggest that persistence through high transfer rates is a costly endeavour that might not always be successful and therefore is not the best long-term strategy (Hall et al. 2017).

Finally, there is some suggestion that the large size of some plasmids may help to explain their persistence. Chromids are very large plasmids (comparable in size to a bacterial chromosome) that are found in about $10 \%$ of bacterial genomes (Harrison et al. 2010). These elements share chromosomal and plasmid characteristics, in that they can carry essential housekeeping genes and share the codon usage properties of a chromosome, while carrying plasmid genes such as replication and partitioning genes, as well as other accessory genes (Harrison et al. 2010; Hülter et al. 2017). The replication of chromids is thought to be coordinated with that of the host chromosome (Hülter et al. 2017).

Essential housekeeping genes may remain on chromids for many reasons. This configuration may allow the host to maintain a larger genome overall, while allowing the chromosome to remain smaller. Under this configuration, genetic material can be more quickly replicated. Moreover, replicative stress on the cell may be reduced, since 
replication can start later during the cell cycle, when cell size and resource availability may be greater. Further, while less evidence supports the following possibility, it is suggested that this configuration allows for the coordinated regulation of different chromid genes when the host adapts to different niches, such that different environments can induce different groups of genes to be expressed or not, depending on the conditions to which the host is exposed. For example, the effect of the loss of a $1.35 \mathrm{Mb}$ megaplasmid and $1.68 \mathrm{Mb}$ chromid was measured in Sinorhizobium meliloti. While the host could survive in the absence of these elements, a limitation in the number of usable carbon sources was observed, which suggested that there may be a limited niche range in the absence of these elements (diCenzo et al. 2014). Further, the loss of the megaplasmid resulted in an increase in growth rate relative to the wild type. Nonetheless, it continues to persist in the face of this cost, suggesting that it must confer some advantage to the host that may not be observed in the laboratory.

\subsection{Conclusions}

Plasmids have clear benefits in a number of challenging environments, encoding traits such as antibiotic resistance, virulence, and metal tolerance. In the absence of selection for such traits, however, plasmids often impose a fitness cost. We have suggested that the mechanisms underlying these fitness costs are incompletely understood; resource use and interference with host processes certainly contribute to particular cases, but the relative importance of these mechanisms across plasmids and hosts is still unclear. Moreover, the evolutionary processes that maintain plasmids in the face of segregational loss, fitness costs, and integration require further investigation. 
Elucidation of the mechanisms underlying costs and of the processes contributing to persistence will both clarify fundamental plasmid biology and assist in the management of plasmid-encoded traits such as antibiotic resistance. 


\section{Chapter 3: Host-plasmid interactions in Escherichia coli and their effect on plasmid maintenance}

\subsection{Introduction}

Bacterial plasmids are generally small extrachromosomal DNA elements which can carry a variety of accessory genes, including an increasing number of antimicrobial (AMR) genes (Rankin et al. 2011; Shintani et al. 2015). As almost half of all plasmids have the ability to horizontally transfer to nearby cells and frequently carry AMR genes, they are a major concern in the fight against increasing AMR and its public health burden (Rozwandowicz et al. 2018). As such, it is essential to understand the spread and persistence of plasmids.

While plasmids carrying AMR genes confer a selective advantage to their hosts in the face of antibiotic selection, they are thought to be a burden to their hosts in the absence of selection, decreasing the plasmid-containing host's competitive ability in mixed populations (Vogwill and MacLean 2015; San Millan and MacLean 2017). Costs can be a result of the trade-offs the host experiences at times when the plasmid is not being selected for; that is, maintaining a costly plasmid might be favourable when the host is being subjected to treatment with an antibiotic to which plasmid confers resistance, but when there are no antibiotics present in the environment, this burden is not offset by the increased chance of survival by the host.

However, these costs are not guaranteed, with several documented instances where no fitness cost is conferred - indeed, plasmid carriage is sometimes beneficial in 
the absence of selection (Enne et al. 2004; Cottell et al. 2012; Humphrey et al. 2012). Moreover, costs can be inconsistent across genetic backgrounds (strains or clones belonging to the same species but differing by some, and possibly many, mutations), with variation in the cost of a given plasmid between hosts (Humphrey et al. 2012; Starikova et al. 2013; Di Luca et al. 2017; Valle et al. 2020). For example, Valle et al (2020) measured the fitness effects of a pOXA-48 plasmid into 50 isolates of Escherichia coli and Klebsiella pneumoniae and found that while on average there was a cost to plasmid carriage, there was a high degree of variability in fitness outcomes across the strains, with 14 genotypes experiencing a cost and 7 being more fit than the plasmid-free host. Further, the availability of hosts for which plasmid carriage is neutral or beneficial can contribute to the persistence of otherwise costly plasmids (e.g., Valle 2020).

This dependence on genetic background is an instance of epistasis, or gene-bygene $(\mathrm{GxG})$ interaction, in which the genotype of the host bacterium partially determines the fitness effects of plasmid carriage (Vogwill and MacLean 2015; Carroll and Wong 2018). Practically, epistasis implies that fitness estimates of a plasmid in one genotype will not necessarily be useful for predicting the cost of the same plasmid in a different host. Instead, the genetic background of the host can directly play a role in a plasmid's fitness and constrain its persistence in different lineages, meaning that treating hostplasmid combinations might differ depending on the combination (Chandler et al. 2013).

Broadly, there are three possible outcomes of plasmid acquisition on the host: plasmid carriage may be costly, neutral, or beneficial in a given environment. In cases where plasmids are costly (as is generally thought to be the case; Vogwill and MacLean 2015), we expect that they will either be lost due to purifying selection, maintained by 
compensatory evolution, or escape loss through conjugation (Harrison and Brockhurst 2012). Costly plasmids should be lost over time as plasmid-free segregants would have an advantage and outcompete plasmid-bearing cells (Silva et al. 2012; Million-Weaver and Camps 2014). However, initially costly plasmids often persist for hundreds of generations in the absence of selection (for example, (Cottell et al. 2012; Fischer et al. 2014; Porse et al. 2016)), suggesting that additional mechanisms contribute to their retention. There is plenty of evidence that compensatory mutations can allow plasmids to remain in the absence of selection (Starikova et al. 2013; Porse et al. 2016; Di Luca et al. 2017). For example, Porse et al (2016) found that selection in media containing antibiotics for $\sim 280$ generations was sufficient to compensate for the fitness effects of the pKP33 plasmid in E. coli in the absence of drug selection, even though pKP33 carries a blaTEM-CTX-15 variant and ten other resistance genes. Similarly, Enne et al (2004) found that E. coli JM109 carrying the p9938 plasmid was initially costly, but could evolve to outcompete its plasmid free ancestor after as few as 20 generations. Further, Hall et al (2020) found that 48 hours, the time it took a transconjugant colony to grow on solid medium, was enough to compensate the cost of an initially costly plasmid, pQBR55, in Pseudomonas fluorescens SBW25.

While there is substantial evidence that compensatory evolution contributes to plasmid maintenance, conjugative transfer rates are typically thought to be too low to account for plasmid maintenance, and when transfer rates are high enough to do so, it becomes so costly as to outweigh the benefit of transfer (Harrison and Brockhurst 2012; MacLean and San Millan 2015; Porse et al. 2016; Hall et al. 2017b). Nonetheless, there is little empirical data on the role of conjugation in the maintenance of costly resistance 
plasmids, although there certainly is evidence for the importance of conjugation in some cases (Lopatkin et al. 2017). For example, a conjugation-deficient mutant of the RP4 plasmid in Serratia marcescens, when subjected to protozoan predation, is unable to persist, suggesting the importance of conjugation in plasmid persistence, at least in some cases (Cairns et al. 2016). Lopatkin et al (2017) found that conjugation happened frequently enough to allow for the plasmids to persist and Stevenson et al (2017) found that with weak mercury selection of pQBR57 in Pseudomonas fluorescens favoured plasmid maintenance by conjugation, rather than through the vertical transfer observed under strong selection. At the community level, conjugation can have a role in plasmid maintenance, however, the host needs to be a proficient plasmid host and diversity is more likely when the plasmid is beneficial (Kottara et al. 2020).

When plasmid carriage is neutral, we expect plasmid frequency to depend on genetic drift, particularly for small plasmids lacking particular segregation systems that rely upon adequate distribution between daughter cells during cell division (MillionWeaver and Camps 2014). Finally, if the plasmid is beneficial, we expect it to sweep to fixation (Bergstrom et al. 2000). That is, because the strain bearing the plasmid is more fit and outcompetes strains without the beneficial genes (either on another plasmid or on the chromosome of the competitors), it increasingly makes up larger proportions of the population. However, if a competitor were to arise where some or all of the plasmid has integrated into the chromosome, this could have the added benefit of maintaining useful plasmid genes while avoiding the potential loss of the plasmid through segregation; if this integration did not occur in such a way that disrupts essential gene function, the cost of maintaining a separate element can be reduced. 
While plasmids are expected to be lost or integrated, depending on whether they are costly or beneficial, they often persist for long periods of time in both the lab and nature (Cottell et al. 2012; Fischer et al. 2014; Porse et al. 2016); this mismatch between expectation and observation has been coined the "plasmid paradox" (Harrison and Brockhurst 2012). Costly plasmids often persist during laboratory selection, as a result of compensatory mutations and increased plasmid control (Harrison and Brockhurst 2012; MacLean and San Millan 2015; Porse et al. 2016; San Millan and MacLean 2017). Compensatory mutations reduce the burden the plasmid presents to the cell, lessening the impact of purifying selection (Harrison and Brockhurst 2012). Integration, on the other hand, is expected in cases where genes residing on the plasmid are positively selected. However, integration is rarely observed in laboratory studies, and in one example where integration of an antibiotic resistance gene occurred, it was shown to not be due to selection by that drug (Modi et al. 1992; Harrison and Brockhurst 2012). Further, integration could result in other issues, such as integrating within or near important chromosomal genes and interfering with their expression, which can be detrimental to the host (San Millan and MacLean 2017). More specifically, when plasmids are able to integrate, some are more likely to integrate in specific locations, such as the Haemophilus plasmid p1056 typically inserting into tRNA genes, reducing the chance of integrating in a detrimental location, whereas others can insert more randomly, which can interrupt the gene(s) into which it is inserted (Dimopoulou et al. 2002; Roberts and Mullany 2009; Johnson and Grossman 2015). Finally, the presence of partitioning systems and postsegregational killing mechanisms (PSK), more typically found on large, low-copy plasmids, can reduce the likelihood of potentially more fit plasmid-free segregants, as 
PSK typically results in the death of plasmid-free segregants (Hernández-Arriaga et al. 2014; Million-Weaver and Camps 2014). Thus, the mechanisms whereby plasmids are retained in populations remain to be fully elucidated.

Here, we examined mechanisms of persistence for a conjugative multidrug resistant plasmid, pPB29, following acquisition by diverse hosts. Given previous studies, we expected that carriage would be generally costly, and that persistence would largely be attributable to compensatory evolution. We instead found that the fitness effects of this plasmid, pPB29, were strongly dependent on the host's genetic background - plasmid acquisition ranged from strongly deleterious to strongly beneficial in the absence of selective antibiotic. For costly host-plasmid combinations, we found limited evidence for compensatory evolution. Rather, contrary to expectations, conjugation was an important mechanism by which pPB29 persisted.

\subsection{Materials and Methods}

\subsubsection{Culture Conditions, Media, and Antibiotic Stocks}

We used six genotypes of $E$. coli, including one lab strain and five clinically derived strains (Table 3.1). All strains were streaked on Lysogeny Broth (LB; 1\% tryptone, $0.5 \%$ yeast extract, $1 \% \mathrm{NaCl}$ ) plates (LB with $1.5 \%$ agar). All plates were incubated inverted overnight ( $20-24$ hours) at $37^{\circ} \mathrm{C}$. Lac ${ }^{-}$derivatives were generated by deleting the Lac operon ( $\triangle l a c$; MG1655 and PB 4), or by inserting a yellow fluorescent protein (YFP) marker in place of the Lac operon ( $\Delta l a c-Y F P$; OLC 682, OLC 969, PB 1 and PB 15; Hinz et al. in preparation). Lac ${ }^{-}$strains were used as plasmid recipients for the 
transconjugants used below. The ancestral $\mathrm{Lac}^{+}$strains were used for competitions to utilize blue-white screening for colony counts. Deletion of the lac operon in MG1655 and PB 4 was completed through homologous recombination using a derivative of the allelic replacement vector pAH79 carrying a resistance gene for tetracycline and protocol modified for E. coli that utilizes WM3064, a donor strain auxotrophic for diaminopimelic acid (DAP) (Saltikov and Newman 2003; Bailey et al. 2014; Lebeuf-Taylor et al. 2019). The lac deletion vector included $\sim 700 \mathrm{bp}$ of sequences upstream and downstream from the lac operon for homologous recombination with E. coli recipients and excluded sequences spanning from the lac promoter to the lacA terminus. Following conjugation with the WM3064 donor, transconjugants were selected on LBA with tetracycline to select transconjugants and without DAP to exclude donor cells. Colonies were then counterselected on media with sucrose and X-Gal+IPTG to select for strains which had excised the vector, and which were Lac-. Strains PB1 $l a c$-YFP, PB15 $l a c-Y F P$, OLC682 $l a c$-YFP, and OLC969 $l a c$-YFP were produced via the same protocol modified for the addition of the YFP marker (Hinz et al. in preparation).

Blue-white screening was carried out on LBA with X-Gal+IPTG plates with XGal at a final concentration of $40 \mu \mathrm{g} / \mathrm{ml}$ and IPTG at $1 \mathrm{mM}$. All samples were diluted using sterile $1 \mathrm{X}$ M9 Salt Solution (prepared by diluting $5 \mathrm{X}$ stock with $\mathrm{dH}_{2} \mathrm{O}$ and autoclaving; $5 \mathrm{X}$ stock is $30 \mathrm{~g} / \mathrm{L} \mathrm{Na} \mathrm{HPO}_{4}, 15 \mathrm{~g} / \mathrm{L} \mathrm{KH}_{2} \mathrm{PO}_{4}, 5 \mathrm{~g} / \mathrm{L} \mathrm{NH} 4 \mathrm{Cl}$, and $2.5 \mathrm{~g} / \mathrm{L}$ $\mathrm{NaCl}$ ). Overnight cultures of $E$. coli were grown in LB for 24 hours at $37^{\circ} \mathrm{C}$, either with or without the presence of antibiotics as required. Kanamycin stocks $(50 \mathrm{mg} / \mathrm{ml}$, diluent $\mathrm{dH}_{2} \mathrm{O}$ ) were prepared, and filter sterilized as required and stored at $-20^{\circ} \mathrm{C}$. Antibiotics were diluted to working concentrations in liquid LB for MICs and culturing. 
Table 3.1: Serotype, infection source, and plasmid information for E. coli strains used in this experiment.

\begin{tabular}{ccccc}
\hline Strain & Serotype & $\begin{array}{c}\text { Infection } \\
\text { Source }\end{array}$ & Known Plasmids & Reference \\
\hline \hline MG1655 & - & - & None & (Bachmann 1972; \\
PB1 & ST-131 & UTI & IncFIB(AP001918),IncFII(pHN7A8) & (Basra et al. 2018) \\
PB4 & ST-1670 & UTI & None & (Basra et al. 2018) \\
PB15 & ST-648 & Respiratory & IncFII(pRSB107), & (Basra et al. 2018) \\
OLC682 & O111:NM & Food & IncFIA,IncFIB(AP001918) & (Markell 2017) \\
OLC969 & O103:H2 & Food & IncFIB (AP001918) & (Markell 2017) \\
& & & & \\
\hline
\end{tabular}

\subsubsection{Plasmid Conjugation}

We used a plasmid from a blood-infection isolate of E. coli ST-315 strain PB29, which will be referred to as pPB29 from now on (Basra et al. 2018). This plasmid belongs to the IncFII family of plasmids, and is known to carry the following resistance genes: aac(2)-IIa, tet(A), blaCTX-M-15, blaOXA-1. The pPB29 plasmid $\left(\mathrm{Amp}^{\mathrm{R}} \mathrm{Kn}^{\mathrm{R}}\right)$ was transferred to six diverse hosts by conjugation. Overnight cultures of donor and recipient were diluted 1:10, grown to early $\log$ phase, and then mixed in a 1:10 ratio of plasmid donor to recipient. The donor strain, $\mathrm{PB} 29$, was $\mathrm{Lac}^{+}$and the recipients were the $\mathrm{Lac}^{-}$versions of MG1655, PB1, PB4, PB15, OLC 682, and OLC 969, allowing for the use of blue-white screening for transconjugants. PB1, PB4, and PB15 are extraintestinal pathogenic E. coli (ExPEC) and OLC 682 and OLC 969 are enterohemorrhagic E. coli (EHEC). Mating mixtures were then either spotted on LB plates and incubated at $37^{\circ} \mathrm{C}$ overnight, or liquid mixtures were kept at $37^{\circ} \mathrm{C}$ overnight without shaking. The following day, a portion of the spots on LB plates were resuspended in $1 \mathrm{~mL}$ of $\mathrm{LB}$, diluted, and plated on selective plates containing $100 \mu \mathrm{g} / \mathrm{ml}$ kanamycin; liquid mating mixtures were diluted and plated on selective plates. Plates were checked the following day for 
transconjugants. Conjugation efficiency was calculated by determining the number of recipient (plasmid-free ancestor) cells in the overnight culture prior to starting conjugation. After 24 hours, the number of transconjugants were measured. Both measurements were done in triplicate. Conjugation efficiency was calculated as the number of transconjugants per recipient cell.

\subsubsection{Minimum Inhibitory Concentration (MIC) Determination}

MIC assays were performed using a two-fold dilution series in 96-well plates and growth noted the following day. The cultures used for these assays were diluted 1:100 from fresh overnight cultures in LB. Note that LB media was used throughout, to be consistent with the media used for the measurement of fitness. The MIC of each strain was measured in duplicate, and a one-way ANOVA was performed to determine if the genetic background of the host was a significant contributor to end-point resistance.

\subsubsection{Measurement of Relative Fitness}

Fitness was measured using head-to-head competitions in mixed culture for each of the six transformants. Overnight cultures of each $\mathrm{Lac}^{-}$transformant and its $\mathrm{Lac}^{+}$ plasmid-free ancestor were inoculated in 200 ul each LB media in triplicate in 96-well microwell plates. The following day, each plasmid-host combination was diluted to a final dilution of 1:100 in $200 \mathrm{ul}$ of LB media in new 96-well plates and mixed well. Aliquots of the cultures at the start $\left(\mathrm{T}_{0}\right)$ and end $\left(\mathrm{T}_{24}\right)$, following 24 hours of growth at $37^{\circ} \mathrm{C}$ with shaking, were frozen at $-80^{\circ} \mathrm{C}$ with glycerol at a final concentration of $25 \%$.

Relative counts for each plasmid-free ancestor and transformant pair for both time points 
were completed using a ten-fold dilution series made from the frozen competition plates in fresh 96-well plates with 1X M9. Twenty-five microliters of each dilution were spotted on X-Gal+IPTG plates which were incubated overnight and spots with countable colonies were counted for analysis. Ancestral colonies were blue on X-Gal+IPTG and transconjugant colonies were white. Relative fitness was calculated using the following equation derived by Dykhuizen and Hartl (1983):

$$
\text { Relative Fitness }=1+\frac{\ln \left(\frac{\text { T24transconjugant }}{\text { T0transconjugant }}\right)-\ln \left(\frac{\text { T24ancestral }}{\text { T0ancestral }}\right)}{\text { number of generations }}
$$

Fitness values were scaled by dividing the relative fitness of the transconjugant to the Lac + plasmid-free ancestor by the relative fitness of the plasmid-free marked strain (Lac-) to the same Lac + plasmid-free ancestor.

\subsubsection{Selection Experiment and Plasmid Loss Enumeration}

Liquid cultures for each of the transconjugants were inoculated in LB from a single colony and incubated at $37^{\circ} \mathrm{C}$ overnight with shaking. The following day, transconjugants were diluted 1:100 in fresh LB in triplicate in a 96-well plate with 198ul of broth in each well. Each replicate was spaced such that there was a control well with only LB in between each replicate as a check for inter-well contamination. The plate was incubated at $37^{\circ} \mathrm{C}$ overnight with $150 \mathrm{RPM}$ shaking, and the following morning was diluted as above for 15 transfers ( $\sim 100$ generations).

After 15 transfers, a sample of each population was streaked for single colonies, and a single colony of each used for competitive fitness assays, as described above. Plasmid loss was estimated by diluting a sample of the frozen stock and plating these 
dilutions on LBA. Plates were grown overnight and the following day, twenty random colonies from each replicate population for each of the six strains were chosen from plates with well-spread colonies. These twenty randomly chosen colonies were patched onto LBA supplemented with X-Gal and IPTG, as well as LBA+X-Gal+IPTG supplemented with either $200 \mu \mathrm{g} / \mathrm{ml}$ ampicillin or $100 \mu \mathrm{g} / \mathrm{ml}$ kanamycin. Growth for each colony on antibiotic-supplemented media was determined the following day. Strains that grew on the antibiotic were presumed to retain the plasmid and those unable to grow or which exhibited extremely impaired growth were presumed to lack the plasmid.

\subsubsection{Mixed-Culture Selection in the Absence of Antibiotic}

In order to assess the possibility of plasmid persistence through conjugative transfer, mixed-culture selection experiments were carried out as for the single-strain selection experiments, except that a mixed culture of $\mathrm{Lac}^{+}$ancestor and $\mathrm{Lac}^{-}$transformant were used to found each population. These strains were diluted 1:200 in each well containing $200 \mu$ of LB in triplicate, so the final concentration was 1:100. A sample of each overnight culture was diluted 1:100 in fresh LB each day for fifteen days, with glycerol stocks made at the start, as well as after seven transfers ( $\sim 50$ generations) and fifteen transfers ( $\sim 100$ generations). Cultures were serially diluted in 1X M9 salts and $20 \mu 1$ spots of $10^{-3}$ and $10^{-4}$ dilutions for the initial mixture and $10^{-5}$ and $10^{-6}$ dilutions of the 50 and 100 generation stocks were plated on LBA supplemented with X-Gal and IPTG. Blue and white colonies were enumerated and then replica plated onto LBA+XGal+IPTG with $100 \mu \mathrm{g} / \mathrm{ml}$ kanamycin for counts of resistant blue and white colonies. To evaluate the proportion of each cell type (susceptible and resistant plasmid-free host (blue 
cells) and susceptible and resistant plasmid donor (white cells)), the number of each cell type was divided over the total number of cells, as counted on the LBA+X-Gal+IPTG plates. This was done for each replicate at all three time points.

\subsubsection{Statistical Analysis}

Statistical analyses were carried out using $R$ (R Core Team, 2019) and RStudio (RStudio Team, 2018). Figures were produced using R package ggplot2 (Wickham, 2016). Muller plots were produced using R package ggMuller (Noble, 2019). One-way ANOVAs were carried out with "host" as the independent variable and measure (Fitness, Fold Change, etc.) as the dependent variable. Tukey's tests were completed to determine pairs which were significantly different from each other, using $\mathrm{p}=0.05$ as the cut-off for significance. Changes in fitness of initial transconjugants were measured using a onesample Student's $t$-test, with $m u$ set to 1 (indicating equal fitness to the ancestor). The significance of change in resistance and fitness following 100 generations of selection was determined by a one-way ANOVA and Tukey's test to evaluate which evolved populations, if any, were significantly different in fitness than the unevolved transconjugant, again, using $\mathrm{p}=0.05$ as the cut-off for significance.

\subsection{Results}

\subsubsection{Genetic Background Effects on Plasmid Carriage}

We introduced a single plasmid into six genetically diverse genotypes of E. coli to determine the extent to which genetic background influences plasmid effects on fitness 
and resistance. pPB29 was introduced by conjugation into a $\mathrm{Lac}^{-}$derivative of each genotype, and we measured effects on kanamycin resistance, an antibiotic towards which pPB29 confers resistance, using MIC assays. Resistance to kanamycin increased 32-fold to 128 -fold, with four strains increasing 32 -fold in resistance, and PB4 and the enterohaemorrhagic strain OLC 969 having a 128-fold increase (Figure 3.1A; one-way ANOVA $F(5,6)=5.76 \mathrm{E}+31, \mathrm{p}=<2 \mathrm{e}-16$ ). A negative relationship (albeit not significantly so) between change in MIC and starting MIC (Figure A1) is suggestive of diminishing returns epistasis, where strains with higher resistance tended to have smaller increases in resistance when compared to strains with lower starting resistance.

To assess the fitness effects of plasmid carriage in the absence of antibiotic, each transformant was competed against its $\mathrm{Lac}^{+}$isogenic plasmid-free ancestor (Figure 3.1B). Since the Lac deletion was itself costly in some cases, each transconjugant's fitness was scaled against the fitness of its plasmid-free Lac- ancestor in competition against the plasmid-free unmarked $\left(\mathrm{Lac}^{+}\right.$) strain (Figure A2). Transconjugants with a fitness value of 1 are equal in fitness to the plasmid-free $\mathrm{Lac}+$ competitor, whereas values above or below one corresponds to the transconjugant being more or less fit than the competitor, respectively. On average, carriage of pPB29 was costly, with an average relative fitness across all backgrounds of $0.907 \pm 0.051$. Notably, there was significant variation amongst genetic backgrounds, with plasmid carriage leading to fitness reductions on some backgrounds, no effects on others, and an increase in fitness on others (Figure 3.1B; oneway ANOVA $F(5,12)=7.414, p=0.0022)$. The most drastic decrease in fitness was for the laboratory strain MG1655, with a fitness of $0.53 \pm 0.08(t(2)=-5.77, p=.028)$. The EHEC strain O111:NM (OLC 682) had the next lowest fitness of $0.85 \pm 0.03(t(2)=-5.16$, 
$p=.035)$. $\mathrm{PB} 1$ and $\mathrm{PB} 15$ were not significantly different from their plasmid-free ancestors. For the other EHEC strain, O103:H2 (OLC969), as well as one of the ExPEC strains (PB4), plasmid carriage conferred a benefit, with transformant fitnesses of $1.06 \pm 0.01$ $(t(2)=7.31, p=.018)$ and $1.06 \pm 0.02(t(2)=4.0522, p=.05585)$, respectively.
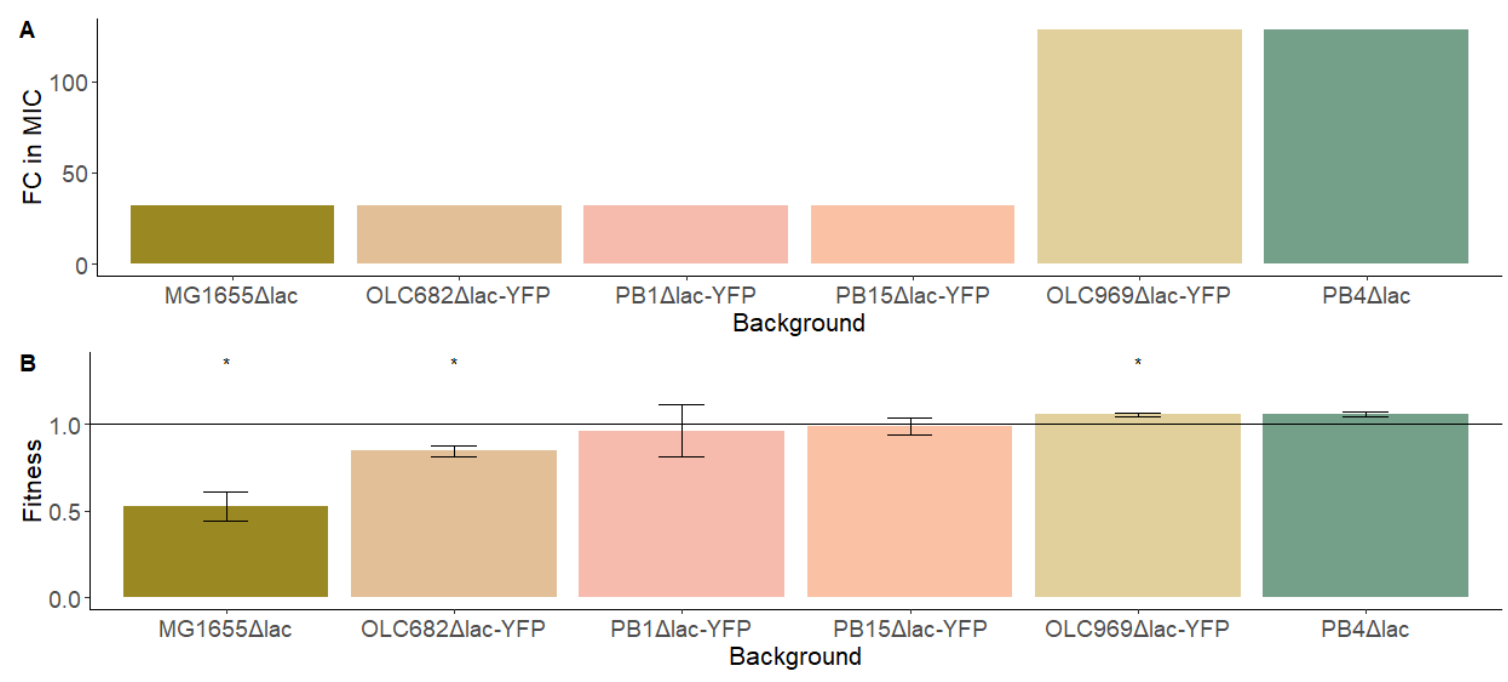

Figure 3.1: Effect of genetic background on the impact of plasmid carriage. A. Fold change in the minimum inhibitory concentration (MIC) of transconjugants towards $\mathrm{Km}$ following acquisition of pPB29 in six genetically distinct E. coli host strains

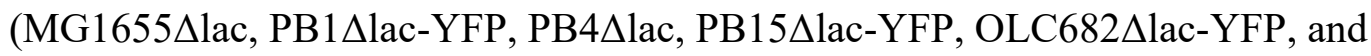
OLC969 4 lac-YFP). B. Relative fitness values due to pPB29 acquisition. Results obtained from mixed culture competition assay of transformants against their plasmid-free ancestors and scaled to competitions between the plasmid-free marked and unmarked strain. Asterisk in (B) denotes genotypes where the fitness is significantly different than 1 (i.e., equally fit to plasmid-free ancestor) as found with a one-sample $t$-test.

Because we assessed ancestor and transformant counts through the use of a chromosomal marker (presence or absence of the lac operon), our fitness estimates could be inaccurate if conjugative transfer occurred over the 24-hour competition period. As such, we determined the conjugation efficiency of each host-plasmid combination against 
their $\mathrm{Lac}^{+}$plasmid-free competitor and found that two strains, four of the strains had conjugation efficiencies below $10^{-3}$, whereas PB1 and PB15 had higher transfer efficiencies (Figure 3.2). Both of these strains were also as fit or more fit than their plasmid-free competitor, so the effect of conjugation is unclear.

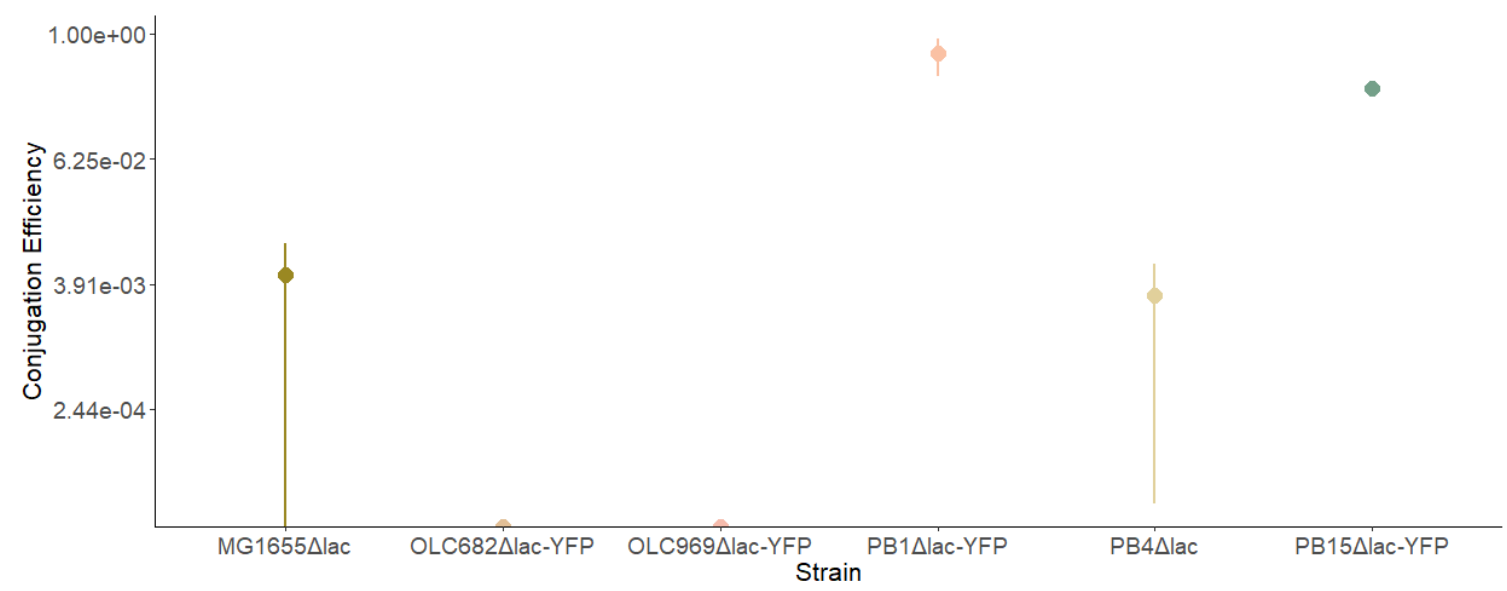

Figure 3.2: Conjugation efficiency of transconjugants. Conjugation efficiency of each transconjugant strain (donor) into their Lac+ plasmid-free ancestor (recipient). Conjugation efficiency measured as the ratio of transconjugants to recipient cells. Error bars indicate standard error of the mean for three replicates.

\subsubsection{Changes in Fitness and Resistance Following Selection in the Absence of}

\section{Antibiotic}

To evaluate the capacity for compensatory evolution to overcome the costs of plasmid carriage (where present), we evolved populations in the absence of antibiotic for approximately 100 generations and measured fitness and resistance following this period (Figure 3.3). Resistance did change in this period for some of the evolved populations, usually by two-fold (Figure 3.3A). Some of these changes resulted in a decrease in resistance below that of the ancestral transconjugant strain. In two populations on the MG1655 $\Delta$ lacZ background, resistance fell two-fold, as did a single population of 
PB4 $l a c+p P B 29$, and two of OLC682 $l a c-Y F P($ One-Way ANOVA, $p<0.05$ ). Of note, these two evolved OLC682 $\triangle l a c-\mathrm{YFP}+\mathrm{pPB} 29$ populations also had a significant increase in fitness over the same 100 generation period (see below).

Overall, 100 generations of selection had little effect on fitness in the absence of antibiotic (Figure 3.3B). For five genetic backgrounds (MG1655, OLC969, PB1, PB4, and PB15), none of three evolved populations experienced a significant change in fitness compared to the ancestral transconjugant. Only the three evolved populations of OLC682 $l a c-Y F P+p P B 29$ are significantly more fit than their ancestral transconjugants. That is, only three out of eighteen evolved populations showed a significant change in fitness. We found no correlation between change in resistance and change in fitness (Figure A3). 


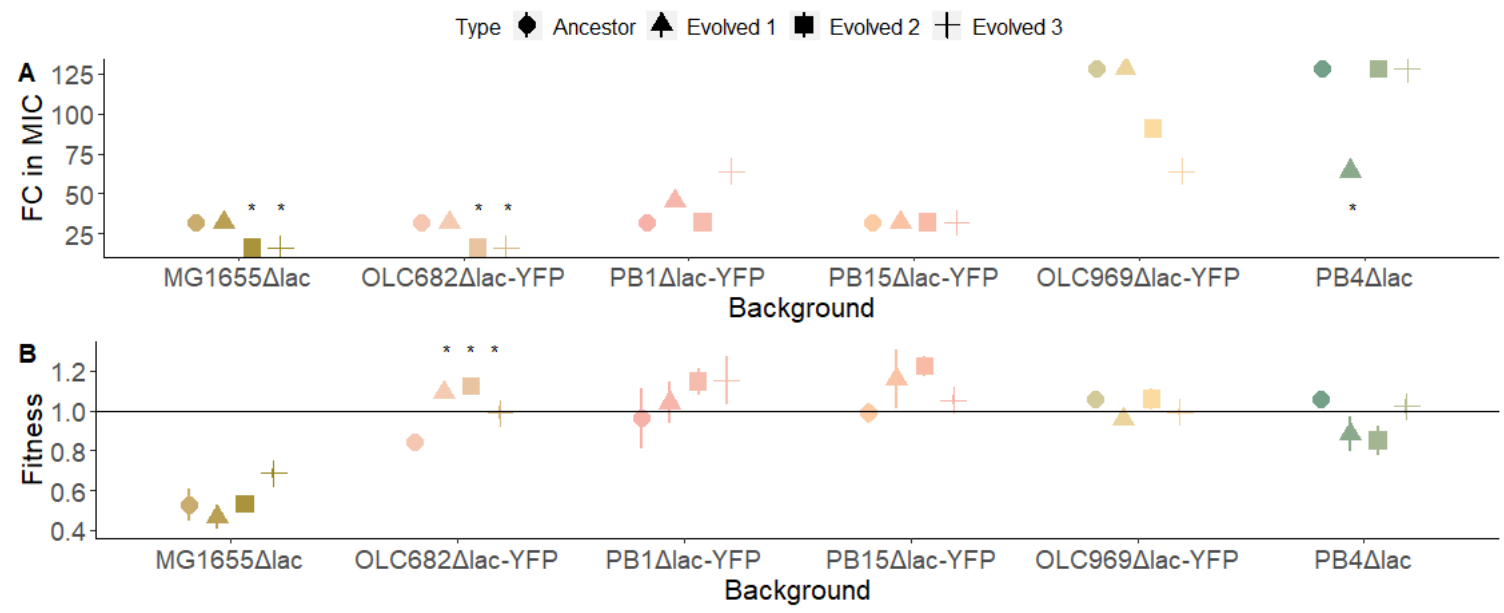

Figure 3.3: Changes in fitness and resistance (minimum inhibitory concentration (MIC)) of three evolved transconjugants following 100 generations compared to the ancestral transconjugant. Changes in resistance and fitness of three replicate populations of each host-plasmid pair following 100 generations of selection in LB. A. fold change in MIC relative to the plasmid-free ancestor of three evolved populations and their unevolved ancestor as measured in LB for all six strains (MG1655 lac, PB1 $l a c-Y F P, P B 4 \Delta l a c$, PB15 $l a c$-YFP, OLC682 $l a c$-YFP, OLC969 $l a c$-YFP) containing the pPB29 plasmid. B. Average relative fitness of three evolved populations and their unevolved ancestor as measured in LB for all six strains containing the pPB29 plasmid.

\subsubsection{Plasmid loss following selection}

To test the prediction that costly plasmids should be lost in the absence of selection, we estimated plasmid prevalence in the evolved populations. The growth of twenty colonies from each evolved population was assessed by replica plating on agar plates supplemented with either ampicillin or kanamycin, drugs for which the pPB29 plasmid carries resistance genes (Table 3.2). This was to confirm loss of resistance to both drugs to which the plasmid confers resistance, suggesting loss of plasmid, rather than just loss of resistance to just one drug, which might suggest the loss of part of the plasmid. Apparent plasmid loss was observed infrequently, as very few colonies lost 
resistance to both drugs. There was no clear pattern in this loss; loss typically happened in a single replicate population of a given genotype, rather than being common in any genotype. A plasmid extraction for each of the colonies that presented less or no growth than from the control plates showed that there was a decrease in plasmid DNA for these isolates when compared to an evolved plasmid-containing strain (Figure A4). Plasmid loss was not more common on genetic backgrounds with a high cost of plasmid carriage (MG1655 and OLC682).

Table 3.2: Plasmid prevalence after 100 generations. Average number of plasmid-bearing colonies from three evolved populations for each of the six transconjugants

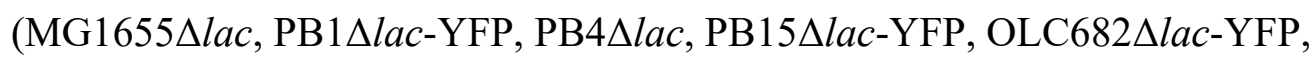
OLC969 $l a c$-YFP with pPB29) following 100 generations of experimental evolution.

\begin{tabular}{|c|c|}
\hline Strain & Plasmid Prevalence (Average out of $20 \pm$ SE) \\
\hline MG1655 $l a c+p P B 29$ & $19.67 \pm 0.33$ \\
\hline $\mathrm{PB} 1 \Delta l a c-Y F P+p P B 29$ & $20.00 \pm 0.00$ \\
\hline $\mathrm{PB} 4 \Delta l a c+\mathrm{pPB} 29$ & $19.67 \pm 0.33$ \\
\hline $\mathrm{PB} 15 \Delta l a c-\mathrm{YFP}+\mathrm{pPB} 29$ & $20.00 \pm 0.00$ \\
\hline OLC682 $2 l a c-Y F P+p P B 29$ & $20.00 \pm 0.00$ \\
\hline OLC969 $l a c-Y F P+p P B 29$ & $19.00 \pm 0.57$ \\
\hline
\end{tabular}

\subsubsection{Plasmid dynamics following mixed-culture selection}

Finally, we examined the dynamics of plasmid loss and transfer during 100 generations of selection in mixed culture. This allowed us to assess the impact that conjugation had on plasmid maintenance, and to see if starting fitness of the transconjugant impacted the importance of conjugation in plasmid maintenance. That is, we could assess whether costly plasmids are frequently lost or transferred to be maintained in the population, or if the plasmid can sweep to fixation when beneficial. We mixed a roughly equal number of the $\mathrm{Kn}^{\mathrm{R}} \mathrm{Lac}^{-}$transconjugant, which is white on $\mathrm{X}$ Gal+IPTG and to which we will refer as the "donor", and the $\mathrm{Kan}^{\mathrm{S}} \mathrm{Lac}^{+}$plasmid-free 
ancestor, denoted the "recipient" and which is blue on X-Gal+IPTG, and passaged mixed populations in LB for 100 generations. Emergence of resistant recipients $\left(\mathrm{Kn}^{\mathrm{R}} \mathrm{Lac}^{+}\right)$ indicates conjugative transfer of pPB29 into the plasmid free ancestor, while susceptible donors $\left(\mathrm{Kn}^{\mathrm{S}} \mathrm{Lac}^{-}\right)$are indicative of plasmid loss. At the start (zero generations), middle (50 generations), and end (100 generations) of the experiment, we estimated the proportion of each of the four types.

Following 100 generations of selection in mixed culture, we see evidence of both plasmid loss from the donor strain, and of transfer to the plasmid-free competitor (Figure 3.4). Fitness of the initial transconjugant predicts, at least in part, the fate of the plasmid. In cases where plasmid carriage provided a benefit (i.e., increased fitness), we predicted that the plasmid would sweep to fixation. Both PB4 and OLC969 transconjugant strains were more fit than their ancestor, OLC969 significantly so (Figure 3.1). For PB4, the resistant donor made up the largest proportion of the population after 100 generations. Some plasmid loss was observed at generation 50 but did not make up a large proportion of the population by the end of the 100 generations (Figure 3.4F). Contrary to expectations, OLC969+pPB29, which was significantly more fit than the plasmid-free ancestor, was rapidly outpaced by both the susceptible and resistant recipient strains, such that the original plasmid donor was not present in the final population in two of the three populations (Figure 3.3E). In cases where the plasmid was initially costly, we expected to see the loss of the resistant donor over time. Plasmid carriage was most costly on the MG1655 and OLC682 backgrounds, and indeed, we observed the loss of the resistant donor quite rapidly; in most cases, the resistant donor was completely lost by 100 generations (Figure 3.4A, Figure 3.4B). Surprisingly, in every case, we saw that the 
plasmid was transferred to the recipient and thereby maintained; in four of the genotypes, the plasmid was only present in the recipient at generation 100, at least in two of the three replicates. However, plasmid-bearing recipients did not necessarily outcompete the susceptible recipients, as they were both frequently found by the end of the 100generation period. Lastly, for neutral host-plasmid combinations we expected the outcome to be a result of drift. On the PB1 background, a mixture of resistant and susceptible clones was observed at generation 100, although the donor genotype remained at very low frequencies (Figure 3.4C). In PB15, where the plasmid was neutral, we see a pattern similar to that of PB4, where the initial plasmid-bearing host makes up the largest proportion of the population by the end of the experiment (Figure 3.4D). Again, there was a clear indication of transfer of the plasmid to the recipient. 

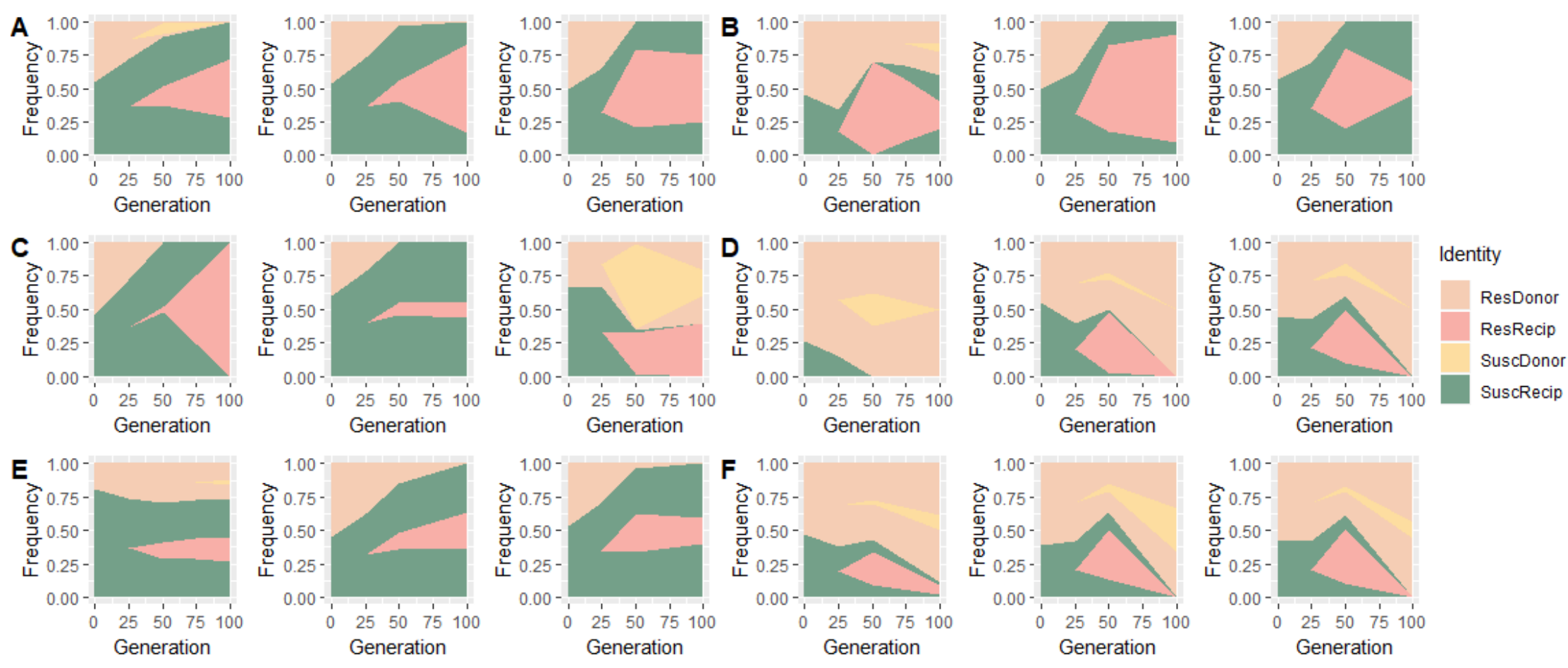

Figure 3.4: Assessment of plasmid dynamics in mixed culture after 100 generations. Changes in resistant and non-resistant ancestral and transconjugant cells over 100 generations of mixed-culture selection for (A) MG1655, (B) OLC682, (C) PB1, (D) PB15, (E) OLC969, (F) PB4. The proportion of each type of cell was measured at start (time=0), 50 generations, and 100 generations of selection when mixed. Each population was started with a mix of resistant donors ("ResDonor") and susceptible recipients (“SuscRecip”). 


\subsection{Discussion}

We aimed to investigate mechanisms of plasmid maintenance following acquisition via conjugation. We assessed the importance of segregating or de novo compensatory mutations, as well as the frequency of both plasmid loss and plasmid transfer. We began our investigation by determining the fitness effect of pPB29, an MDR plasmid of clinical origin, in six genetically distinct isolates of $E$. coli. Following initial measures of fitness and resistance, we evolved isogenic or mixed populations of the transconjugants for 100 generations to assess plasmid dynamics over time. We thus comprehensively tested predictions for plasmid persistence, whereby plasmids are expected to be lost, compensated, or transferred when costly, be subject to drift when neutral, and sweep to fixation when beneficial.

Conjugation rates are thought to be either too low to maintain a costly plasmid, with high transfer rates thought to be too costly because of energetic demands (Bergstrom et al. 2000; Hall et al. 2017b). In contrast to these expectations, we found that conjugation was a common mechanism of persistence when plasmid carriage was costly: in mixed culture experiments, transfer was common, and indeed rescued the plasmid from extinction in several populations (Figure 3.4). Cases where the plasmid was maintained on the recipient background may be the result of hitchhiking (described by Bergstrom et al (2000), and discussed in Chapter 2) with beneficial mutations on the initially plasmid-free background (Bergstrom et al. 2000), although further work would be required to test this hypothesis. Our findings thus contribute to a growing body of evidence suggesting that conjugation can maintain plasmids, both within a lineage and in communities (Lopatkin et al. 2017; Kottara et al. 2020). 
Although plasmid loss is a possible outcome, particularly for costly plasmids, (Bergstrom et al. 2000; Million-Weaver and Camps 2014; Harrison et al. 2016) plasmid loss was infrequent in our monoculture experiments (Table 3.2). Notably, loss was not observed frequently in any strain, and no more frequently in strains for which carriage was costly (Table 3.2). Some loss was noted in our mixed culture experiments (susceptible donors in Figure 3.4), but typically at low frequencies. We note that pPB29 encodes the pemK and pemI toxin and antitoxin genes. Such genes are a part of a plasmid post-segregational killing system, in which the plasmid encodes an unstable antitoxin and a stable toxin; loss of the plasmid results in the loss of the antitoxin, and plasmid-free segregants are killed or have their growth impaired by the toxin (Hernández-Arriaga et al. 2014). As such, loss of plasmid is not expected to be common, due to the removal or reduction of plasmid-free segregants by the toxin when the antitoxin is absent (Hernández-Arriaga et al. 2014). The pemK/I system may therefore explain the infrequent loss of pPB29, even when costly. There is some evidence that the components of these systems can be secreted into the medium, for example, the antitoxin MqsR in $X$. fastidiosa, which may explain how some plasmid-free cells could be viable, though further research would be needed (Shidore and Triplett 2017). Further, because all but MG1655 carry one or more plasmids, it is possible that a similar antitoxin system in another plasmid may act against a toxin until it is degraded, or the toxin only impairs growth, rather than killing the cells, until the toxin is removed.

Our findings also suggest a role for segregating genetic variation in plasmid maintenance. We find that $\mathrm{pPB} 29$ tends to be costly on average to the host: mean fitness relative to the $\mathrm{pPB} 29$-free ancestor was $0.91( \pm 0.08)$, similar to the fitness costs typical of 
AMR plasmids (0.91 0.024; Vogwill and MacLean 2015). Nonetheless, pPB29 was not always costly, and was in fact beneficial on some genetic backgrounds (Figure 3.1). Previous studies have also indicated that the fitness of a plasmid varies depending on host genetic background, including RP1 in E. coli (Humphrey et al. 2012), vanA plasmids in E. faecium (Starikova et al. 2013), pG12-KPC-2 in E. coli (Di Luca et al. 2017), and pOXA-48 in E. coli and K. pneumoniae (Valle et al. 2020). Overall, this variability indicates that we likely would not be able to predict the fitness outcome for any other host-pPB29 combination and would need to test this each time.

Host variation in plasmid fitness effects indicates that some genetic backgrounds already possess mutations making plasmid carriage less deleterious. Costs associated with plasmid carriage are thought to be primarily a result of increased metabolic burden and immediate lack of control of plasmid gene expression. Cost reductions may then result from increased regulation (Vogwill and MacLean 2015; San Millan and MacLean 2017). Similarly, costs imposed by widespread gene misregulation in the well characterized pBQR103 system (Harrison et al. 2013) are ameliorated by mutations that restore normal patterns of transcription. Therefore, it is possible that the clinical isolates, particularly the PB hosts which already possess at least one other plasmid (Basra et al. 2018), may have encountered similar plasmids in their past, and thus already possess mutations which ameliorate the costs of related plasmids. Similarly, it has been found that the cost of carrying multiple plasmids can be reduced through positive epistasis (San Millan et al. 2014a). Consistent with this hypothesis, the laboratory strain MG1655 does not carry any other plasmids and experienced the greatest decline in fitness; perhaps its 
naivety and lack of other plasmids both contribute to the steep decline in fitness, when compared to the other strains.

Compensatory variation can also arise de novo, and as such we asked if 100 generations of selection in LB would result in compensation in costly host-plasmid pairs (Figure 3.3B). Here, we found evidence for compensatory evolution only in populations OLC682 $\Delta l a c+Y F P$ strain; fitness increases were accompanied by reduced kanamycin resistance in two populations (Figure 3.3A). Loss of AMR genes have been reported as a mechanism of cost amelioration following coevolution, though since there is only a decrease in resistance in this case, rather than complete loss, regulation of the plasmid might be the overall mechanism of cost reduction (Dahlberg and Chao 2003). Unexpectedly, we did not see fitness improvements in MG1655, even though initial costs of pPB29 carriage were higher in this host. Increased mutation rates in OLC682 (McCarthy et al., in preparation) may explain its higher rate of adaptation.

The "plasmid paradox" articulated by Harrison and Brockhurst (2012) suggests that when costly, plasmids should be lost, or if indispensable, be integrated into the chromosome. Compensatory evolution of initial costs of plasmid carriage has been observed repeatedly in the laboratory, and may well contribute to plasmid persistence (Enne et al. 2005; Starikova et al. 2013; San Millan et al. 2014a,b; Harrison et al. 2015; Porse et al. 2016; Di Luca et al. 2017). We note that the results of compensatory evolution studies are mixed, in that over periods of 100-300 generations of selection, evolved populations can become nearly as fit as the wild-type (Starikova et al. 2013), become slightly worse (San Millan et al. 2014), or even more fit than the wild-type (Enne et al. 2004). Over longer time scales, (400-1100 generations), fitness can be almost fully 
restored, and can even exceed ancestral fitness by up to 70\% (Dahlberg and Chao 2003; Dionisio et al. 2005a; Starikova et al. 2013; Di Luca et al. 2017).

In our system, 100 generations of selection may have been insufficient to observe substantial changes in fitness for most genotypes when evolved in monoculture; many of the evolved populations showed small, albeit non-significant, increases in fitness. Moreover, since several of our ancestral transconjugants were already nearly as fit as or more fit than their ancestors, selection for compensatory mutations would be weak or non-existent. This is not something that was accounted for at the start of the experiment; many studies that show compensatory evolution for host-plasmid pairs show this compensation can occur rapidly, although some of the examples discussed in Chapter 2 show compensation following 300 generations. Perhaps this shows that it is more likely that compensatory evolution will be observed after this period, and that changes in fitness might actually be slower for some genotypes and higher in others when it does occur. The monoculture selection experiment could have been run for longer, however, the results from the mixed-culture experiment do show that 100 generations was enough to observe the changes in frequencies of donors and recipients, so this period of selection can still have utility.

Diminishing returns epistasis, whereby adaptation is slower in already welladapted genotypes, is a frequent observation in laboratory selection experiments (Orr 2005; Weinreich et al. 2006; Chou et al. 2011). Thus, we expected that genotypes with higher levels of antibiotic resistance would show smaller gains in resistance upon plasmid gain when compared to those with lower resistance (Wünsche et al. 2017). We saw a slight, though insignificant, trend of strains with higher starting resistance having smaller 
gains in resistance and vice versa (Appendix A; Figure A1). While this relationship was not significant due to our small sample size, it does suggest that being highly adapted (i.e., highly resistant) can limit the benefit that the acquisition of pPB29 might confer, when compared to less adapted (i.e., less resistant) strains.

From this work, we find that both compensatory mutations and conjugation are important mechanisms for plasmid maintenance within populations. Compensatory variation, both segregating and de novo, are important mechanisms for mediating fitness costs (Harrison and Brockhurst 2012; MacLean and San Millan 2015; Wong 2017). That is, mutations which already have occurred can be responsible for the initial variation in fitness of an initial host-plasmid combination (Figure 3.1B), and ones that occur after can help compensate for new costs associated with plasmid carriage as observed with the significantly more fit evolved populations of OLC 682 following 100 generations of evolution (Figure 3.3B) (Wong 2017). Conjugation was also an important mechanism for plasmid maintenance, and despite arguments that plasmid transfer can become uncontrollable and thus parasitic, there is some evidence to suggest that a costly plasmid can be maintained by conjugation. Conjugative transfer can also act more broadly in communities to preserve useful plasmids or to provide a reservoir for the plasmid when it cannot be maintained in the initial host (Bergstrom et al. 2000; Hall et al. 2016; Lopatkin et al. 2017; Carroll and Wong 2018; Kottara et al. 2020). Notably, the combination of host genetic variation and conjugative transfer increases the likelihood of plasmid persistence; a plasmid that finds itself in a costly host may survive not only by compensatory evolution, but by transfer to more favourable hosts. Further studies will be necessary to determine if these patterns and mechanisms of compensation and transfer are 
evident in a broader range of host-plasmid combinations, as well as within various relevant environments, such as those similar to where the strains were isolated from. Further, the longer-term implications of transfer dynamics and fitness should be monitored, as it will not only be interesting and of use to help resolve the plasmid paradox, but it will aid in discovering solutions to plasmid-mediated AMR. 


\section{Chapter 4: Effect of Variable Long-Term Selection on Plasmid Maintenance and Parallel Evolution in Escherichia coli}

\subsection{Introduction}

Plasmids, particularly conjugative or mobilizable antimicrobial resistance (AMR) plasmids, are a major concern in part because they can be rapidly disseminated within populations, and may persist for long periods of time (Bergstrom et al. 2000; Dahlberg and Chao 2003; Fischer et al. 2014). It is essential to understand the conditions under which plasmids will continue to be maintained, and for how long, since AMR plasmids can give their host an advantage in the presence of drugs for which the plasmid encodes resistance.

These plasmids frequently, but by no means always, incur a fitness cost in their hosts, and the fate of these costly plasmids can depend on selection, as well as compensatory evolution, and conjugation (Bergstrom et al. 2000; Harrison and Brockhurst 2012; Vogwill and MacLean 2015). The "Plasmid Paradox" has been introduced to highlight how initially costly plasmids tend to persist beyond expectation through these mechanisms despite theory predicting plasmid loss through purifying selection or integration of important genes (Harrison and Brockhurst 2012; but see Chapter 2 for a more thorough discussion).

Given the costliness associated with many plasmids, selection may be required for long-term plasmid persistence. Selection experiments, in which hosts bearing an AMR plasmid are passaged for several generations with or without antibiotic, provide mixed messages regarding this expectation. For instance, in shorter-term selection experiments, pCT and pCT2 in E. coli (Cottell et al. 2012), pKP33 in E. coli and K. pneumoniae (Porse 
et al. 2016), pB10 in some of the Alpha-, Beta-, and Gammaproteobacteria hosts (De Gelder et al. 2007), and pG12-KPC-2 and pG06-VIM-1 in E. coli (2017) were able to persist for 70, 200, 280, and 312 generations, respectively, without selection by antibiotics. However, antibiotic selection was required to maintain some plasmids over time, such as pNUK73, which was able to persist in Pseudomonas aeruginosa PAO1 for 300 generations only when antibiotic selection was present (San Millan et al. 2014b). Fewer studies have investigated plasmid persistence on longer time scales, although selection is not always required in these cases either (Dahlberg and Chao 2003; Dionisio et al. 2005; Starikova et al. 2013; Di Luca et al. 2017). For example, Dionisio et al (2005), Starikova et al (2013), and Harrison et al (2015) measured plasmid persistence for around 420, 400, and 450 generations, respectively, and Dahlberg and Chao (2003) measured plasmid stability over 1100 generations. In the last example, the plasmid was found to be stable over this period, as no plasmid-free clones were detected at the end of the period.

Parallel evolution occurs when the same trait is evolved independently in replicate populations as a response to the same conditions or environment (Futuyma et al. 1995; Schluter et al. 2004). Parallel evolution has been take as evidence of adaptation when the evolved trait appears in multiple independently evolved lineages (Schluter et al. 2004; Woods et al. 2006; Crozat et al. 2010; Bailey et al. 2015, 2017). Parallel evolution has been measured as both a response of the organism to certain environments, as well as in response to plasmid carriage in various selection environments. For instance, there is evidence that parallelism is more likely in lineages of Pseudomonas fluorescens selected in the same, rather than different, environments (Bailey et al. 2015). However, in a case 
where parallel evolution was tested using Pseudomonas fluorescens carrying plasmid pQBR103, Harrison et al (2015) found that varying levels of mercury (Hg) selection resulted in a high degree of parallelism, despite this parallelism occurring between, rather than within, selection conditions. In the latter case, this parallelism might be attributable to varying degrees of the same kind of selection, versus discrete, varied environments with different resources or varied arrangement of resources. In the example with pQBR103, the level of selection in this experiment was ultimately used to change the fitness of the plasmid, as costs are diminished as mercury concentrations are increased. However, starting fitness does not necessarily influence the probability of observing parallel evolution (Bailey et al. 2015). That is, in cases where the plasmid became more fit, this does not necessarily mean that the probability of observing parallel evolution will change.

The types of mutations observed following selection experiments with plasmids can also help point to the source of plasmid-related costs, as the mechanism of compensation may occur in a gene or set of genes that are otherwise impacted by the presence of the plasmid. For example, Yano et al (2016) found that a mutation to the protein $\operatorname{TrfA} 1$, which is a protein responsible for helping with replication of the plasmid, increased persistence of the plasmid. Plasmid replication has been suggested as one possible source for plasmid fitness costs via the sequestration of host-cell machinery for the replication and expression of plasmid genes (San Millan and MacLean 2017). An example of mutations to genes which control the expression of genes as a response to a costly plasmid will be discussed below. Nonetheless, these mutations not only serve to inform us about the potential reason(s) for costliness, in that the observed mutations can 
be related to processes that may be impacted by plasmid acquisition, and thus arose to combat this impact, but these mutations can also be used to investigate the degree of parallel evolution within and between selection conditions.

Mutations which will occur frequently in multiple lineages will be especially interesting, as these mutations might indicate particularly adaptive solutions to costly plasmid carriage. Molecular techniques, such as whole genome sequencing, have allowed for a direct assessment of both compensatory and parallel evolution. One study observed the potential for parallel evolution with respect to a plasmid-bearing strain of Pseudomonas fluorescens (Harrison et al. 2015). They measured the fitness of six replicate populations of $P$. fluorescens SBW25 with pQBR103 in various selection conditions $(0-40 \mu \mathrm{M}$ mercury) for $\sim 450$ generations and found that fitness both improved and that persistence was common when mercury was $8 \mu \mathrm{M}$ or higher. They report changes to gacA/gacS, a global regulatory system, across conditions and replicates, which led to the gene expression of many chromosomal and plasmid genes being downregulated. Such changes are not surprising, as gene mis-regulation upon acquisition has been suggested as a mechanism for plasmid costs, so increased regulation within the cell may improve fitness overall (San Millan and MacLean 2017). However, with respect to the work done by Harrison et al (2015) the effect of genetic background on these trends was not assessed, particularly where the plasmid has variable costs in different hosts; this is important, as this can highlight if the cost associated with the plasmid (when there is a cost) is the same between genotypes, and if similar strategies of compensatory evolution are more likely because of this. 
To determine the impact of genotypic and environmental variation on the probability of parallel evolution, I evolved three strains of Escherichia coli carrying the multidrug resistant plasmid pPB29 for 500 generations in three selection conditions, with no drug, $0.1 \mu \mathrm{g} / \mathrm{ml}$, and $10 \mu \mathrm{g} / \mathrm{ml}$ kanamycin. Following experimental evolution, fitness in antibiotic-free and antibiotic-containing media was measured, as were plasmid stability and parallel evolution. Based on the results from Chapter 3, I predicted that fitness would generally increase over time. Further, I predicted that populations selected in LB with kanamycin for 500 generations will be more fit than those selected in LB when fitness is measured in higher concentrations of kanamycin. I predicted that plasmid loss would not be observed frequently, particularly not when the plasmid is selected for by kanamycin, such that I would expect more loss when the plasmid is costly and there is no benefit of the plasmid by providing resistance to the drug. End-point populations and their unevolved ancestors were sequenced to identify any instances of parallel evolution, as well as any genes which may be responsible for increased fitness following 500 generations. I predicted that I would see some level of parallel evolution, particularly on broader measures, such as fitness and the types of genes where mutations occurred, such that there may be mutations related to the same processes, and that similar strategies for improving fitness would be observed across genetic backgrounds in response to pPB29. 


\subsection{Materials and Methods}

\subsubsection{Culture Conditions, Media, and Antibiotic Stocks}

I used three genotypes of $E$. coli, including a lab strain (K-12 derivative MG1655), one clinically derived from a UTI (PB1), and one from a food-borne infection (OLC 969) (Table 4.1). All strains were streaked on Lysogeny Broth (LB; 1\% tryptone, $0.5 \%$ yeast extract, $1 \% \mathrm{NaCl}$ ) plates (LB with $1.5 \%$ agar). All plates were incubated inverted overnight ( $20-24$ hours) at $37^{\circ} \mathrm{C}$. $\mathrm{Lac}^{-}$derivatives were generated by deleting the Lac operon ( $\Delta l a c$; MG1655), or by inserting a yellow fluorescent protein (YFP) marker in place of the Lac operon ( $\Delta l a c-Y F P$; PB 1 and OLC 682). Lac ${ }^{-}$strains were used as plasmid recipients for the transconjugants used in these experiments. The ancestral $\mathrm{Lac}^{+}$strains were used for competitions to utilize blue-white screening for colony counts when competitions were done in LB. For competitions carried out in kanamycin, $\mathrm{Lac}^{+} \mathrm{MG} 1655$ was transformed with the pET-28 vector, which confers resistance to kanamycin. The deletions of the lac operons for the host strains (MG1655, PB1, and OLC969) were carried out as described in Chapter 3.

Blue-white screening was carried out on LBA with X-Gal+IPTG plates with XGal at a final concentration of $40 \mu \mathrm{g} / \mathrm{ml}$ and IPTG at $1 \mathrm{mM}$. All samples were diluted using sterile $1 \mathrm{X}$ M9 Salt Solution (prepared by diluting $5 \mathrm{X}$ stock with $\mathrm{dH}_{2} \mathrm{O}$ and autoclaving; $5 \mathrm{X}$ stock is $30 \mathrm{~g} / \mathrm{L} \mathrm{Na}_{2} \mathrm{HPO}_{4}, 15 \mathrm{~g} / \mathrm{L} \mathrm{KH}_{2} \mathrm{PO}_{4}, 5 \mathrm{~g} / \mathrm{L} \mathrm{NH} 4 \mathrm{Cl}$, and $2.5 \mathrm{~g} / \mathrm{L}$ $\mathrm{NaCl}$ ). Overnight cultures of $E$. coli were grown in LB for 24 hours at $37^{\circ} \mathrm{C}$, either with or without the presence of antibiotics as required. Kanamycin stocks $(50 \mathrm{mg} / \mathrm{ml}$, diluent 
$\mathrm{dH}_{2} \mathrm{O}$ ) were prepared, and filter sterilized as required and stored at $-20^{\circ} \mathrm{C}$. Antibiotics were diluted to working concentrations in liquid LB for MICs and culturing.

Table 4: Serotype, infection source, and plasmid information for strains of E. coli used.

\begin{tabular}{ccccc}
\hline Strain & Serotype & $\begin{array}{c}\text { Infection } \\
\text { Source }\end{array}$ & Known Plasmids & Reference \\
\hline \hline MG1655 & - & - & None & (Bachmann 1972; Guyer \\
et al. 1981) \\
PB1 & ST-131 & UTI & IncFIB(AP001918),IncFII(pHN7A8) & Basra et al. 2018 \\
OLC969 & O103:H2 & Food & IncFIB & (Markell 2017)
\end{tabular}

\subsubsection{Plasmid Conjugation}

The strains used in these experiments were initially constructed and used for experiments in Chapter 3. The plasmid was from the donor PB29, and this plasmid will be referred to as pPB29 from now on. This plasmid belongs to the IncFII incompatibility group, and carries genes conferring resistance to aminoglycosides (aac(2)-IIa), $\beta$-lactams (blaCTX-M-15 and blaOXA-1), and tetracycline (tetA). pPB29 was conjugated into the three host strains by taking overnight cultures of the donor and recipients, diluting these 1:10 in LB, and growing to early log-phase. The plasmid donor and recipient were then mixed in a 1:10 ratio. These mating mixtures were either spotted on LB plates or left in liquid culture without shaking overnight at $37^{\circ} \mathrm{C}$. The following day, spots on LB plates were resuspended in LB, diluted, and plated on selective plates with $100 \mu \mathrm{g} / \mathrm{ml}$ kanamycin; liquid mating mixtures were similarly diluted and plated. These selective plates were assessed the following day for transconjugants. 


\subsubsection{Selection Experiment and Plasmid Loss Enumeration}

Liquid cultures for each of the transconjugants were inoculated in LB from a single colony and incubated at $37^{\circ} \mathrm{C}$ overnight with shaking. The following day, transconjugants were diluted 1:100 in fresh LB, either on its own or supplemented with $0.1 \mathrm{ug} / \mathrm{ml}$ or $10 \mathrm{ug} / \mathrm{ml}$ kanamycin, in triplicate in a $96-$ well plate with $198 \mathrm{ul}$ of broth in each well. Each replicate was spaced such that there was a control well with only LB in between each replicate as a check for inter-well contamination. The plate was incubated at $37^{\circ} \mathrm{C}$ overnight with $150 \mathrm{RPM}$ shaking, and the following day, each sample was diluted 1:100 as above for 76 transfers ( $\sim 500$ generations). A sample of each population was streaked onto LBA supplemented with X-Gal and IPTG every 50 generations to ensure that populations were not contaminated with $\mathrm{Lac}+E$. coli, or any other observable contaminants which had different morphologies or colours.

After 76 transfers, a sample of each population was streaked for single colonies, and a single colony of each was used for competitive fitness assays. Plasmid loss was estimated by plating dilutions of the frozen stocks on LBA and then picking sixteen wellisolated colonies to streak on LBA supplemented with X-Gal and IPTG, as well as LBA+X-Gal+IPTG supplemented with $100 \mu \mathrm{g} / \mathrm{ml}$ kanamycin or $150 \mu \mathrm{g} / \mathrm{ml}$ ampicillin. Growth for each colony on antibiotic-supplemented media was determined the following day. Strains that grew on plates with both antibiotics were presumed to retain the plasmid and those unable to grow or which exhibited extremely impaired growth were presumed to lack the plasmid. 


\subsubsection{Measurement of Relative Fitness}

Fitness was measured using head-to-head competitions in mixed culture for each of the representatives from each evolved and unevolved population. Overnight cultures of each $\mathrm{Lac}^{-}$transformant and its $\mathrm{Lac}^{+}$plasmid-free ancestor was inoculated separately in $200 \mathrm{ul}$ each LB media in triplicate in 96-well microwell plates. For competitions carried out in LB with kanamycin, overnight cultures of each $\mathrm{Lac}^{-}$transformant and the common competitor MG1655+pET-28 were inoculated in the appropriate medium. The following day, each ancestor-transconjugant combination was diluted to a final dilution of 1:100 in $200 \mathrm{ul}$ of LB media in new 96-well plates and mixed well. Note that depending on the competition environment, either LB without antibiotics, LB with $10 \mu \mathrm{g} / \mathrm{ml}$ kanamycin (1X MIC; concentration is $1 \mathrm{X}$ the ancestral MIC), and LB with $100 \mu \mathrm{g} / \mathrm{ml}$ kanamycin (10X MIC; concentration is $10 \mathrm{X}$ the ancestral MIC), was used. Aliquots of the cultures at the start $\left(\mathrm{T}_{0}\right)$ and end $\left(\mathrm{T}_{24}\right)$, following 24 hours of growth at $37^{\circ} \mathrm{C}$ with shaking, were frozen at $-80^{\circ} \mathrm{C}$ with glycerol at a final concentration of $25 \%$. Relative counts for each plasmid-free ancestor and transformant pair for both time points were completed using a ten-fold dilution series made from the frozen competition plates in fresh 96-well plates with 1X M9. Twenty-five microliters of each dilution were spotted on X-Gal+IPTG plates which were incubated overnight and spots with countable colonies were counted for analysis. Ancestral colonies were blue on X-Gal+IPTG and transconjugant colonies were white. Relative fitness was calculated using the following equation derived by Dykhuizen and Hartl (1983):

$$
\text { Relative Fitness }=1+\frac{\ln \left(\frac{\text { T24transconjugant }}{\text { T0transconjugant }}\right)-\ln \left(\frac{\text { T24ancestral }}{\text { T0ancestral }}\right)}{\text { number of generations }}
$$


Fitness values were scaled by dividing the relative fitness of the transconjugant to the Lac+ plasmid-free ancestor by the relative fitness of the plasmid-free marked strain (Lac-) to the same Lac + plasmid-free ancestor.

Statistical analyses were completed on fitness to assess significant differences within and between replicates and conditions. Changes between replicates or between selection and competition conditions were assessed using an analysis of variance (ANOVA) and a Tukey's post-hoc test to determine which replicates or conditions were significantly different from each other. Linear regression models were utilized to determine if fitness changed over time for each genotype separately, using the following equation: Fitness $\sim$ Time + Replicate + Time*Replicate.

\subsubsection{Sequencing}

A single colony was grown in LB overnight for each of the evolved populations (500 generations) and the ancestral transconjugants, and a genomic extraction was performed for each using the One-4-All Genomic DNA Miniprep Kit (BioBasic BS88503). Samples were then sent to the Microbial Genome Sequencing Center (MiGS) in Pittsburgh, USA for paired-end sequencing (2x150bp) using the Illumina NextSeq 550 sequencing platform. Bioinformatic analysis was completed using several publicly available programs. The quality of reads was determined with FastQC (Andrews 2010) and suboptimal reads were removed using Trimmomatic to improve the accuracy in further steps (Bolger et al. 2014). Reads were trimmed using the following parameters: LEADING:3, TRAILING:3, SLIDINGWINDOW:4:30, and MINLEN:36. A draft genome assembly of each ancestral genotype was assembled using the de novo assembler 
SPAdes with default settings (Bankevich et al. 2012) and annotated using RAST (Aziz et al. 2008). Genetic changes in the evolved populations were found using the Snippy tool (Seemann 2015) using the Genbank formatted annotated reference genome and the forward and reverse fastq read files obtained from MiGS. Manual analysis of the resulting variants was conducted to assess mutations which occurred repeatedly between replicates, selection conditions, and genotypes. Jaccard's Index $(\mathrm{J})$, a measure which can be used to indicate the overlap of mutations between two or more categories, was also calculated using the following equation:

$$
J(X, Y)=\left(\frac{|X \cap Y|}{|X \cup Y|}\right) \times 100
$$

\subsection{Results}

\subsubsection{Fitness of Evolved Populations in the Absence of Antibiotic Selection}

Following 500 generations of selection either with or without sub-MIC levels of kanamycin, I measured changes in fitness in the absence of drug. Fitness for MG1655 $l a c+\mathrm{pPB} 29$ and PB1 $l a c-\mathrm{YFP}+\mathrm{pPB} 29$ evolved in the three selection environments significantly increased over the 500 generations measured (Figure 4.1). Using a repeated measured ANOVA for each genotype (Fitness $\sim$ Generation*Selection Condition +Error(Replicate/(Generation*Selection Condition)) ), I found that fitness significantly increased over 500 generations, regardless of selection condition for both of these genotypes ( $p$-value $<0.05$ ). However, despite OLC969 not appearing to change significantly over time, using the above model, there was a significant difference in fitness following 500 generations ( $\mathrm{p}$-value $<0.05$ ). Most, but not all, of the replicate 
populations within each selection environment reached similar endpoint fitness. All endpoint populations of MG1655, except for Kn0.1-selected R2 compared to R1 and R3 in the same condition, were not significantly different than each other (One-way ANOVA; Fitness $\sim$ Replicate for each selection condition-genotype pair, $p<0.05$ ). However, PB1 showed that there was no significant difference in end-point fitness between replicates in each condition, meaning that all replicates reached the same fitness (One-way ANOVA; Fitness $\sim$ Replicate for each selection condition-genotype pair, $\mathrm{p}>0.05)$. Despite there being no significant difference in fitness between 0 and 500 generations for OLC969 (Figure 4.1C), it is evident that most replicates showed an increase and then levelling off for fitness around 200 generations. Most replicates reached a similar fitness, except for Kn10-selected OLC969, R1 was found to be significantly more fit than R2 and R3 (Oneway ANOVA, Tukey's post-hoc test, p-adj $=0.046(\mathrm{R} 2), 0.029(\mathrm{R} 3))$.

Notably, I did not find a significant difference in end-point fitness, as measured in LB, between the three selection conditions for any of the three genotypes I tested (Figure 4.1). This means that selection in the presence or absence of antibiotic, at least for the sub-MIC levels tested here, do not introduce a significant difference in fitness between the evolved populations when measured in LB. Note that these competitions in LB were between a clone from each evolved population and their respective lac + ancestor, which will vary from the competitions presented in the next section, where a lac+ common competitor (MG1655+pET28) was used for all competitions. 

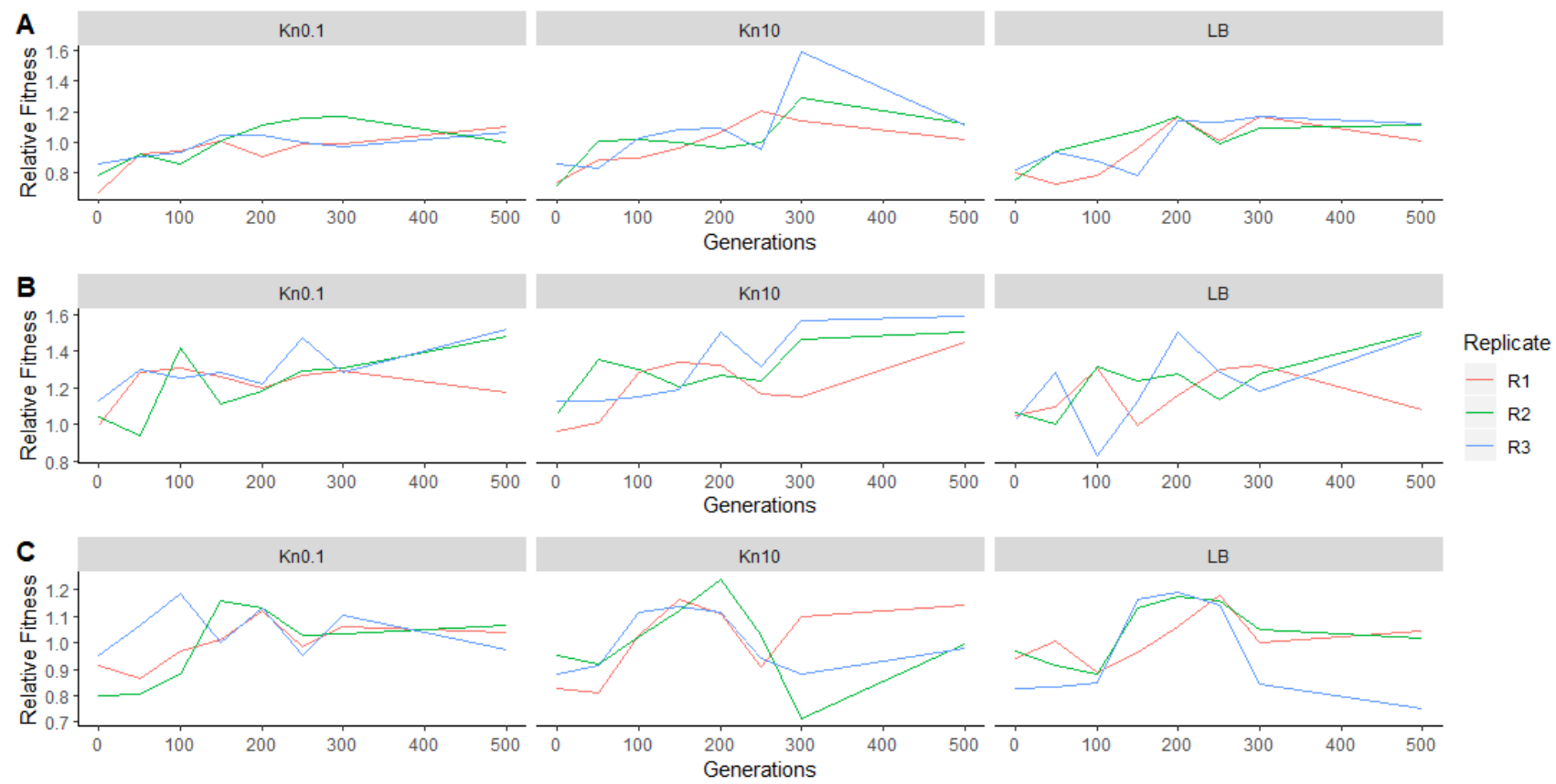

Figure 4.1: The relative fitness in LB of three host-plasmid combinations following evolution with and without kanamycin selection for the plasmid over 500 generations.

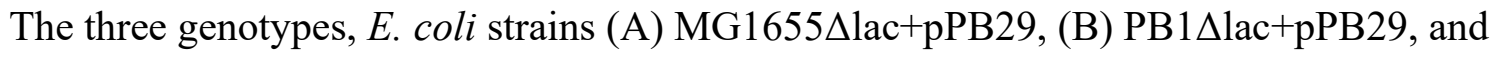
(C) OLC969 $\mathrm{lac}+\mathrm{pPB} 29$, were evolved in LB or LB with 0.1 or $10 \mu \mathrm{g} / \mathrm{ml}$ kanamycin for 500 generations. Fitness measured in head-to-head competition assays in LB without antibiotic using a clone from each population every $\sim 50$ generations, which was competed against its relevant plasmid-free ancestor. A two-way repeated measures ANOVA was used to assess the significance of the number of generations and selection condition on fitness; fitness changed significantly for each genotype over 500 generations $(p<0.05)$, but selection condition was not significant $(p>0.05)$.

\subsubsection{Fitness of Evolved Populations in Three Competition Environments}

Competitions for all evolved populations were undertaken in LB media with either no kanamycin (LB), or kanamycin at $1 \mathrm{X}$ ancestral MIC $(10 \mu \mathrm{g} / \mathrm{ml}$ kanamycin; referred to as $1 \mathrm{X}$ MIC from now on) and 10X ancestral MIC (100 $\mu \mathrm{g} / \mathrm{ml}$ kanamycin; referred to as 10X MIC from now on). Here, I used a common competitor, 
MG1655+pET28, which was resistant to kanamycin, since the isogenic $\mathrm{Kn}^{\mathrm{S}}$ competitors used in 4.3.1 performed poorly in $1 \mathrm{X}$ and $10 \mathrm{X} \mathrm{Kn}$. I did these competitions to observe whether selection environment had an impact on the trajectory of fitness over time, and to see if different selection conditions would affect the fitness in varied competition environments. More specifically, would fitness be higher at $100 \mathrm{ug} / \mathrm{mL} \mathrm{Kn}$ for populations selected at higher, but still sub-MIC, concentrations of drug? I predicted that fitness would be increased when I evolved the populations in kanamycin rather than in LB without the antibiotic.

For competitions carried out in LB without antibiotic in this set of competitions, fitness increased for MG1655 following 500 generations of evolution in the three selection environments (Figure 4.2A; Two-way repeated measures ANOVA; Fitness Generation*Selection Condition +Error(Replicate/(Generation*Selection Condition)), $\mathrm{p}<0.05$ ). However, fitness for PB1 (Figure 4.2B) OLC969 (Figure 4.2C) does not change significantly following 500 generations (Two-way repeated measures ANOVA; Fitness Generation*Selection Condition +Error(Replicate/(Generation*Selection Condition)), pvalue $>0.05$ ). A pairwise t-test with Bonferroni correction comparing genotype to fitness confirmed that MG1655 was significantly different that PB1 and OLC969 in the LB competition environment ( $\mathrm{p}$-adj. $<0.05$ ). Fitness between populations selected in LB, $\mathrm{Kn} 0.1$, or $\mathrm{Kn} 10$ in the LB competitions was not significantly different, except for Kn0.1 and Kn10-selected OLC969 (Pairwise t-test with Bonferroni correction, p-adj<0.05). 

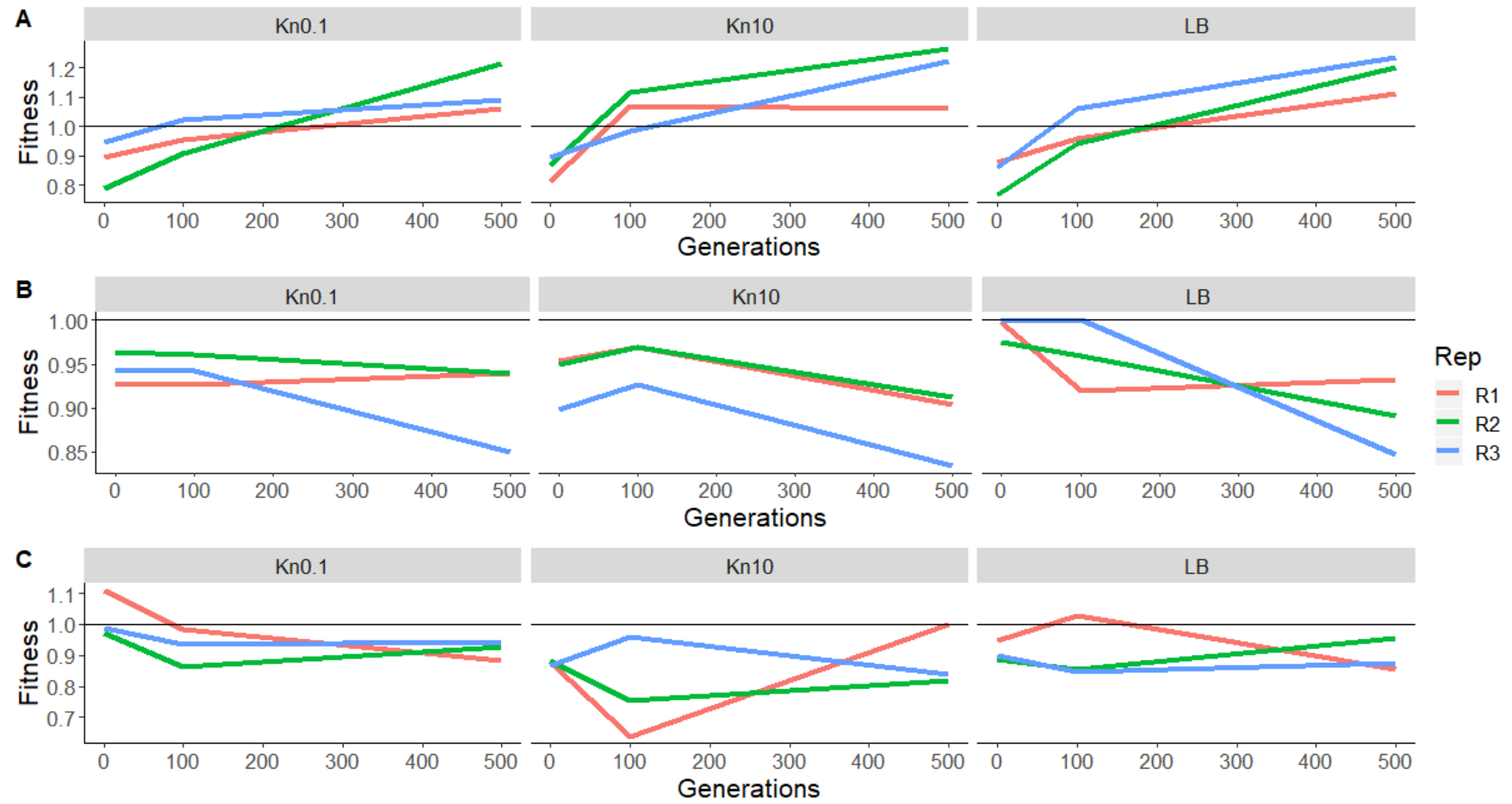

Figure 4.2: The relative fitness in LB of three host-plasmid combinations compared to the common competitor MG1655+pET28 following evolution with and without kanamycin selection for the plasmid over 500 generations. The three genotypes, E. coli strains (A)

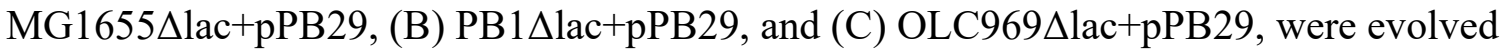
in LB or LB with 0.1 or $10 \mu \mathrm{g} / \mathrm{ml}$ kanamycin for 500 generations. Fitness measured in head-to-head competition assays in LB without antibiotic using a clone from each population every $\sim 50$ generations, which was competed against MG1655+pET28. A twoway repeated measures ANOVA was used to assess the significance of the number of generations and selection condition on fitness; fitness changed significantly for MG1655 over 500 generations, and the only difference in fitness for the three selection conditions was for OLC969 in the Kn0.1 and Kn10 selection environments.

Similar to competitions in LB-only, fitness in 1X ancestral MIC kanamycin increased for MG1655, remained the same for OLC969, and decreased in PB1 (Figure 4.3; Two-way repeated measures ANOVA; Fitness $\sim$ Generation*Selection Condition 
+Error(Replicate/(Generation*Selection Condition)), p-value for MG1655 and PB1 < 0.05 , p-value for OLC969>0.05). There was no significant different in fitness between the three selection conditions for any of the three genotypes assessed (Pairwise t-test with Bonferroni correction, p-adj. > 0.05). Of the genotypes, the fitness of MG1655 and OLC969 was significantly different from each other following the selection experiment (Pairwise t-test with Bonferroni correction, $\mathrm{p}$-adj.<0.05). Note that fitness for PB1 generally decreased over 500 generations, regardless of selection condition or competition condition (Figures 4.2B, 4.3B, and 4.4B). This was not expected, as fitness for PB1 increased significantly following 500 generations in the initial competitions done in LB and is contrary to my expectations that fitness should increase over time (Figure 4.1). 

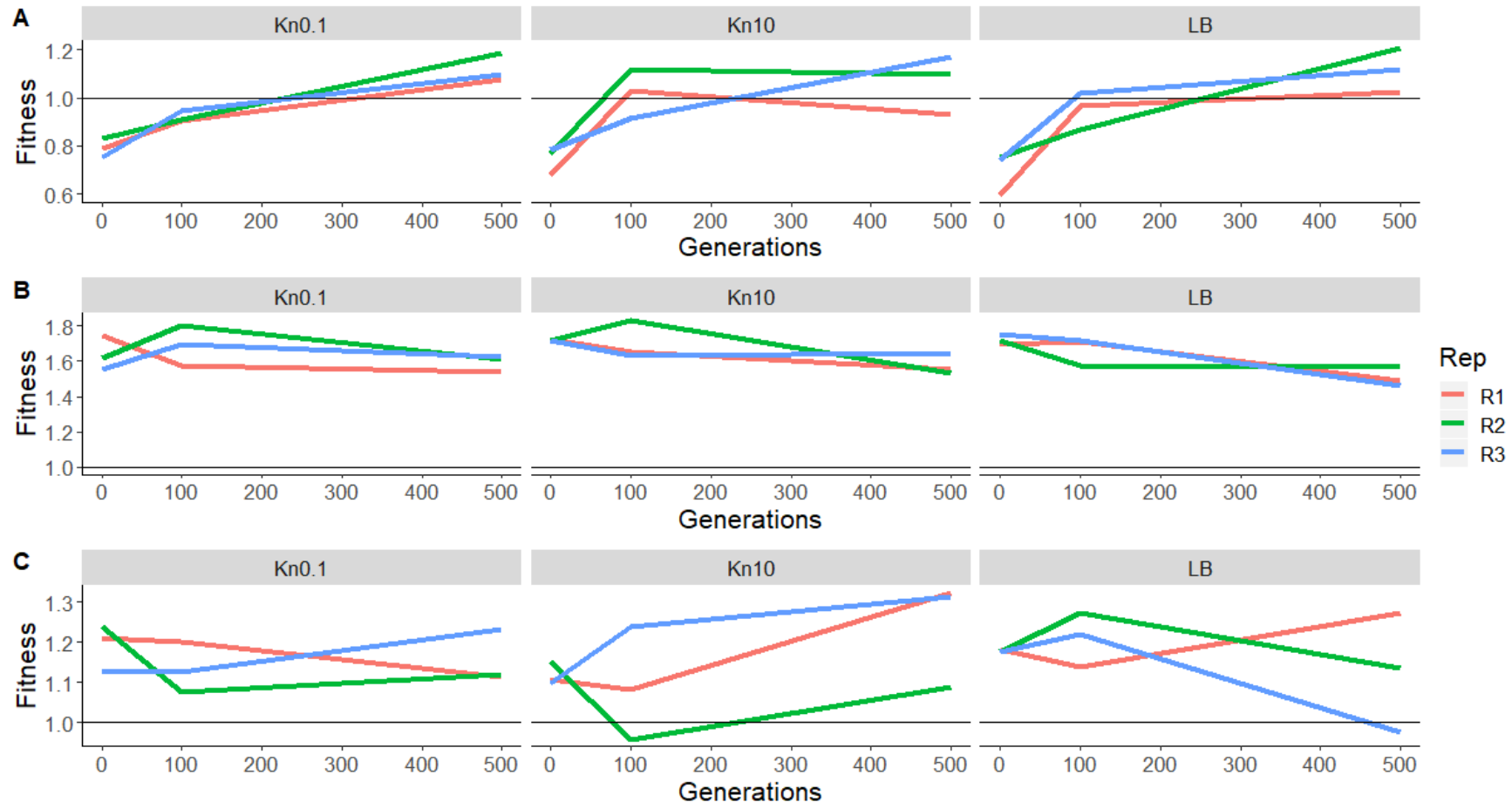

Figure 4.3: The relative fitness in LB with $10 \mu \mathrm{g} / \mathrm{ml}$ kanamycin for three host-plasmid combinations compared to the common competitor MG1655+pET28 following evolution with and without kanamycin selection for the plasmid over 500 generations. The three genotypes, E. coli strains (A) MG1655 $1 \mathrm{lac}+\mathrm{pPB} 29$, (B) PB1 $\Delta \mathrm{lac}+\mathrm{pPB} 29$, and (C) OLC969 $\triangle \mathrm{lac}+\mathrm{pPB} 29$, were evolved in LB or LB with 0.1 or $10 \mu \mathrm{g} / \mathrm{ml}$ kanamycin for 500 generations. Fitness measured in head-to-head competition assays in LB with antibiotic $(10 \mu \mathrm{g} / \mathrm{ml})$ using a clone from each population every $\sim 50$ generations, which was competed against MG1655+pET28. A two-way repeated measures ANOVA was used to assess the significance of the number of generations and selection condition on fitness; fitness changed significantly for MG1655 and PB1 over 500 generations, with fitness significantly increasing and decreasing, respectively.

Finally, for competitions carried out in 10X ancestral MIC kanamycin (100 $\mu \mathrm{g} / \mathrm{ml})$, there was no general trend where fitness apparently increased following 500 generations of evolution (Figure 4.4). The number of generations was not found to change fitness significantly over the period measured here, confirming the graphical data 
(Two-way repeated measures ANOVA, Fitness $\sim$ Generation*Selection Condition +Error(Replicate/(Generation*Selection Condition)), $\mathrm{p}>0.05)$. Fitness did not vary significantly depending on the selection environment for PB1 or OLC969, though the fitness of MG1655 was significantly different between the populations evolved in LB and Kn0.1 (Pairwise t-test with Bonferroni correction, $\mathrm{p}$-adj. $<0.05$ ). In this case, the fitness of all three genotypes was found to be significantly different from each other (Pairwise t-test with Bonferroni correction, $\mathrm{p}$-adj. $<0.05)$. 

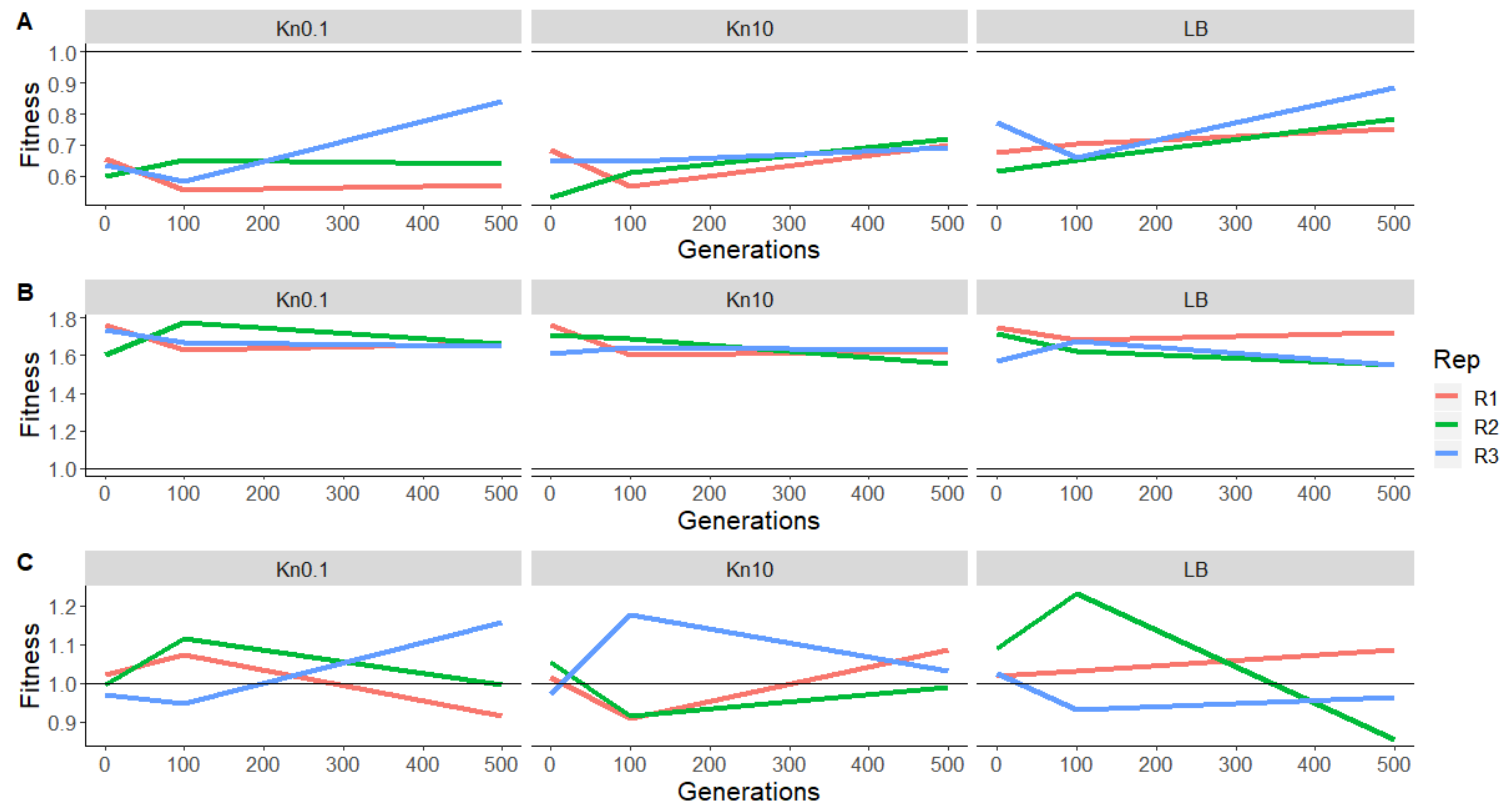

Figure 4.4: The relative fitness in LB with $100 \mu \mathrm{g} / \mathrm{ml}$ kanamycin for three host-plasmid combinations compared to the common competitor MG1655+pET28 following evolution with and without kanamycin selection for the plasmid over 500 generations. The three genotypes, E. coli strains (A) MG1655 $\Delta \mathrm{lac}+\mathrm{pPB} 29$, (B) PB1 $\Delta \mathrm{lac}+\mathrm{pPB} 29$, and (C) OLC969 4 lac +pPB29, were evolved in LB or LB with 0.1 or $10 \mu \mathrm{g} / \mathrm{ml}$ kanamycin for 500 generations. Fitness measured in head-to-head competition assays in LB with antibiotic $(100 \mu \mathrm{g} / \mathrm{ml})$ using a clone from each population every $\sim 50$ generations, which was competed against MG1655+pET28. A two-way repeated measures ANOVA was used to assess the significance of the number of generations and selection condition on fitness; fitness did not change significantly for any genotype following 500 generations of evolution, and the only difference in fitness for the three selection conditions was for MG1655 in the LB and Kn0.1 selection environment. 


\subsubsection{Plasmid Loss Following Experimental Evolution}

I was interested in finding if pPB29 persisted over the course of the selection experiment, and to observe if antibiotic selection would improve plasmid stability over time. Plasmid loss was not found to occur frequently and did not appear to follow any pattern (Table 4.2; three-way ANOVA of Generation x Condition x Replicate for plasmid-carrying colonies, $\mathrm{p}>0.05$ ). That is, plasmid loss was not any more common under conditions lacking selection for plasmid-encoded AMR (LB only) than Kncontaining environments. Plasmid loss was not observed in any Kn-containing condition for $\mathrm{PB} 1 \Delta l a c-\mathrm{YFP}+\mathrm{pPB} 29$ across the four time points assayed, indicating that the plasmid is persisting well in this genotype. However, I saw a slight decrease in plasmid prevalence for MG1655 $\mathrm{Mlac}+\mathrm{pPB} 29$ in the LB and Kn10 selected populations.

OLC969 $l a c-Y F P+p P B 29$ readily maintained the plasmid, however there was some loss in $\mathrm{LB}$ at 100 and 300 generations and at 100 generations in $10 \mathrm{ug} / \mathrm{ml}$ kanamycin. I expected that if plasmid loss were to occur, it would be most common in the LB-selected populations, followed by those selected in Kn0.1, with the Kn10 selected populations having the fewest instances of plasmid loss. Apart from LB-selected MG1655 $l a c+p P B 29$, all populations maintained the plasmid at 500 generations, suggesting an overall pattern of stability of the plasmid over longer periods of experimental evolution regardless of selective conditions. Further, this shows that while plasmid-free variants can arise over time, they do not overtake the plasmid-bearing cells in these cases, especially as fitness of the evolved clones increases relative to the plasmid-free ancestor. 
Integration of plasmid genes was not detected following 500 generations either; since resistance to both antibiotics was either maintained or lost together for all clones, I inferred that kanamycin selection did not result in the integration of that resistance determinant over time. In these cases, I would have expected resistance to kanamycin but not to ampicillin, but all clones which maintained kanamycin resistance were also resistant to ampicillin and if one was lost, so was the other. This conclusion was strengthened from the sequencing data (discussed below), where no large-scale insertions of plasmid genes were detected in a single clone of the populations selected in the three conditions for each genotype. While this only assesses a single clone instead of sixteen, it provides some confirmation for the phenotypic results presented here.

Table 4.2: Prevalence of plasmid loss for MG1655 $l a c+p P B 29$, PB1 $l a c-Y F P+p P B 29$, and OLC969 $l a c-Y F P+$ pPB29 selected in LB, LB with $0.1 \mathrm{ug} / \mathrm{ml}$ kanamycin, and LB with $10 \mathrm{ug} / \mathrm{ml}$ kanamycin from samples frozen at $0,100,300$, and 500 generations. Values are the average of eight colonies streaked on selective and non-selective media and the error represented by the standard error of the mean (SE).

\begin{tabular}{|c|c|c|c|c|}
\hline \multirow{3}{*}{ Strain } & \multirow{3}{*}{ Generations } & \multicolumn{3}{|c|}{ Plasmid Prevalence (Average out of $16 \pm \mathrm{SE}$ ) } \\
\hline & & \multicolumn{3}{|c|}{ Selective Condition } \\
\hline & & LB & $0.1 \mathrm{ug} / \mathrm{ml} \mathrm{kan}$ & $10 \mathrm{ug} / \mathrm{ml} \mathrm{kan}$ \\
\hline \multirow[t]{4}{*}{ MG1655 $l a c+\mathrm{pPB} 29$} & 0 & $16.00 \pm 0.00$ & $16.00 \pm 0.00$ & $15.67 \pm 0.33$ \\
\hline & 100 & $16.00 \pm 0.00$ & $16.00 \pm 0.00$ & $15.67 \pm 0.33$ \\
\hline & 300 & $16.00 \pm 0.00$ & $16.00 \pm 0.00$ & $16.00 \pm 0.00$ \\
\hline & 500 & $15.33 \pm 0.67$ & $16.00 \pm 0.00$ & $15.67 \pm 0.33$ \\
\hline \multirow[t]{4}{*}{$\begin{array}{c}\text { PB1 } 1 \text { lac- } \\
\text { YFP+pPB29 }\end{array}$} & 0 & $16.00 \pm 0.00$ & $16.00 \pm 0.00$ & $16.00 \pm 0.00$ \\
\hline & 100 & $16.00 \pm 0.00$ & $15.33 \pm 0.67$ & $16.00 \pm 0.00$ \\
\hline & 300 & $16.00 \pm 0.00$ & $16.00 \pm 0.00$ & $16.00 \pm 0.00$ \\
\hline & 500 & $16.00 \pm 0.00$ & $16.00 \pm 0.00$ & $16.00 \pm 0.00$ \\
\hline \multirow[t]{4}{*}{$\begin{array}{l}\text { OLC969 } 1 \text { lac- } \\
\text { YFP+pPB29 }\end{array}$} & 0 & $16.00 \pm 0.00$ & $16.00 \pm 0.00$ & $16.00 \pm 0.00$ \\
\hline & 100 & $15.67 \pm 0.33$ & $16.00 \pm 0.00$ & $15.67 \pm 0.33$ \\
\hline & 300 & $16.00 \pm 0.00$ & $16.00 \pm 0.00$ & $16.00 \pm 0.00$ \\
\hline & 500 & $16.00 \pm 0.00$ & $16.00 \pm 0.00$ & $16.00 \pm 0.00$ \\
\hline
\end{tabular}




\subsubsection{Evidence of Parallel and Compensatory Evolution}

Following 500 generations of experimental evolution in all conditions (LB, 0.1 $\mu \mathrm{g} / \mathrm{ml}$ kanamycin, and $10 \mu \mathrm{g} / \mathrm{ml}$ kanamycin), a colony from each end-point population was sequenced, and the mutations in these populations were found by comparing the sequence of the evolved transconjugant to the ancestral one.

There were more mutations found in PB1 than in either MG1655 or OLC969 (Figure 4.5; two-way ANOVA, Number of Mutations Genotype+Selection Condition with Tukey's posthoc test, $\mathrm{p}$-adj<0.05). However, there was no significant difference between the number of mutations found in the different genotypes, nor in the different selection conditions (Two-way ANOVA, Genotype x Selection Condition; adj. p-value > 0.05). In a comparison of the number of mutations found between replicate populations, I found that there were more mutations for R3 of PB1 than there were in R2 (two-way ANOVA, Number of Mutations $\sim$ Genotype + Selection Condition, $p$-adj $<0.05$ ). While not significant, populations evolved in LB had more mutations than those evolved in $\mathrm{Kn} 0.1$ and Kn10. Prior to conducting these experiments, I expected that a genotype which started out less fit would have the greatest number of mutations. However, this does not seem to be the case here, as plasmid carriage was costly for MG1655 prior to the selection experiment (Figures 4.1 and 4.4), but fewer mutations were observed than for PB1.

Five types of mutations were found (Figure 4.6). Missense mutations were frequently observed across genotype and selection conditions, but synonymous mutations were common in PB1 and LB-selected OLC 969. However, there were no significant differences in the number or types of mutations observed across the various selection 
conditions and genotypes (Two-way ANOVA, Genotype x Selection Condition; adj. pvalue $>0.05)$

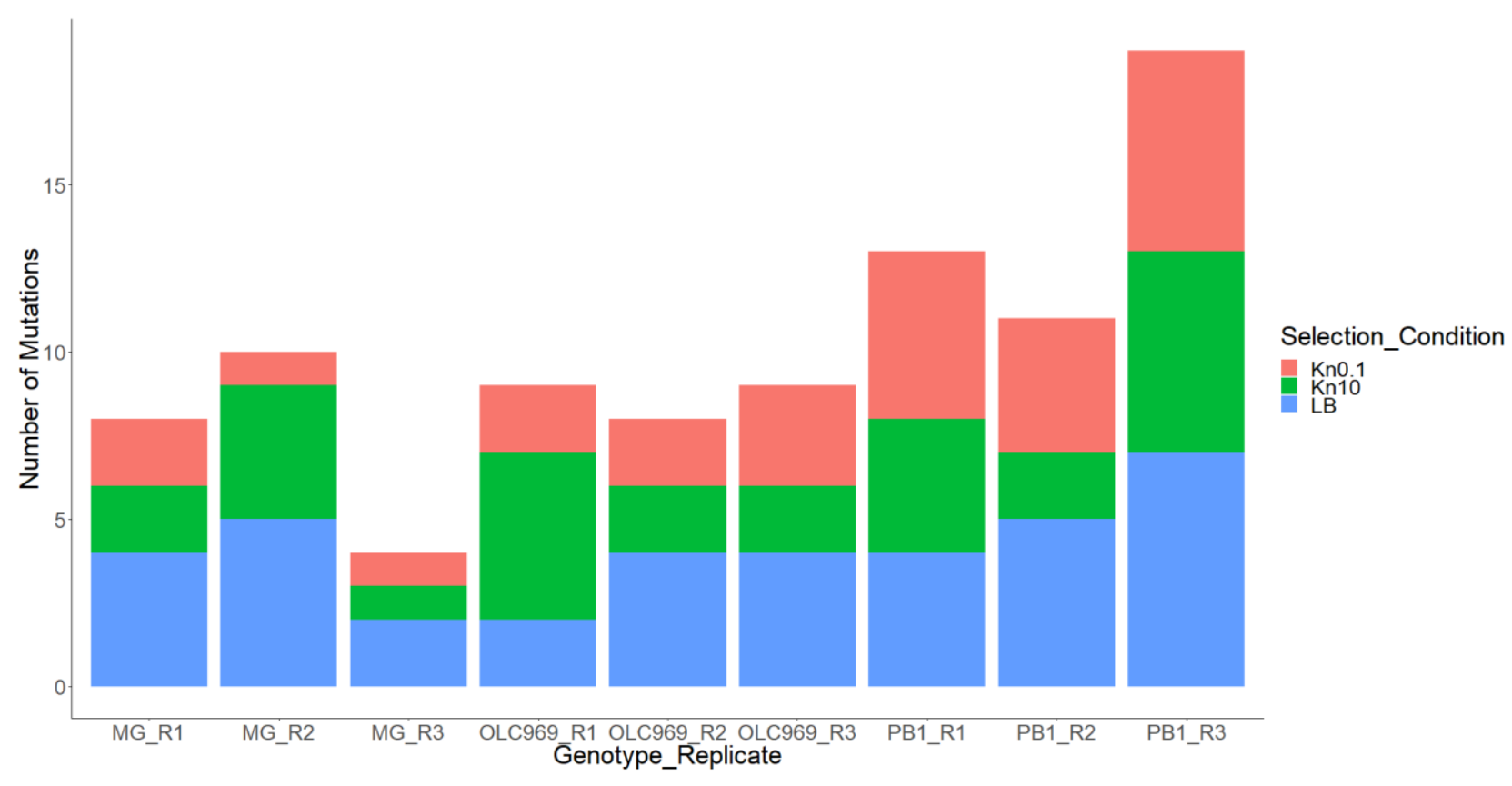

Figure 4.5: The number of mutations found for each clone in each genotype (MG1655, PB1, and OLC969), separated by the selection condition they were found in ( $\mathrm{Kn} 0.1$, Kn10, and LB). PB1 had significantly more mutations than MG1655 or OLC969 (Oneway ANOVA; Number of Mutations $\sim$ Genotype, $\mathrm{p}<0.05$ ), but there was no significant difference in the number of mutations between selection conditions (Two-way ANOVA; Number of mutations $\sim$ Genotype+Selection Condition, $p$-value $>0.05$ ). There were no significant differences between replicate populations of the same genotype, except for PB1 R3 having a significantly higher number of mutations relative to PB1 R2 (Two-way ANOVA; Number of mutations $\sim$ Genotype+Replicate, Tukey's posthoc $p$-adj $<0.05$ ). 


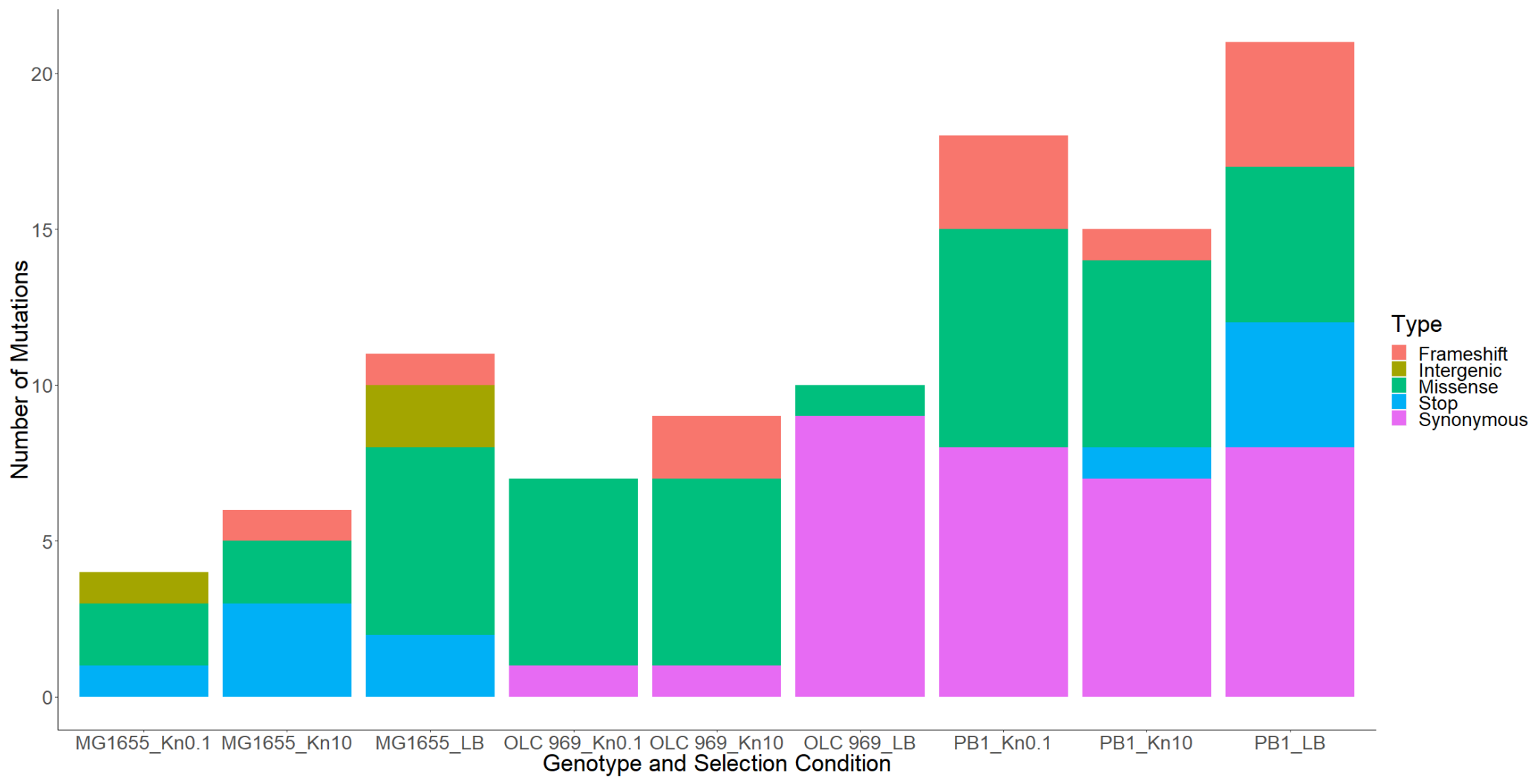

Figure 4.6: The number of mutations per type (frameshift, intergenic, missense, stop, and synonymous) found in each of the genotype and selection condition Following analysis of sequencing data for one clone per replicate population in each of the three selection conditions ( $\mathrm{LB}, \mathrm{Kn} 0.1$, and $\mathrm{Kn} 10$ ) for each genotype, the number of mutations belonging to each type of mutation, was assessed. MG1655 shows a high frequency of missense, followed by stop mutations, whereas more missense and synonymous mutations were observed for PB1 and OLC969. There was no significant difference in the number or types of mutations across conditions or genotypes (One-way ANOVA, $\mathrm{p}>0.05$ ). 
Adaptive mutations can be a result of general adaptation to the culture medium (LB with or without drugs in this case), or mutations which compensate for costs associated with plasmid carriage. Therefore, it is important to distinguish between these types of mutations to be able to discuss what might be a mutation responsible for compensation, rather than a response to growth in LB. Changes to genes related to transcription were found most often (Figure 4.7). Since PB1 started out as approximately neutral, it is likely that most of the mutations which accumulated over time were a response to the culture medium, and not necessarily a response to compensating plasmid costs. In this case, I saw mutations to 3',5'-cyclic-nucleotide phosphodiesterase (EC 3.1.4.17), $\operatorname{lrh} A$, and $\operatorname{traD}$ (Figure 4.7). These genes are responsible for nucleic acid reactions, transcription regulation, and an IncF conjugative transfer gene, respectively. However, MG1655 with pPB29 started out with a cost, and mutations to tRNA-Gln-CTG (LB and $\mathrm{Kn} 0.1), d p p C$ and $r p o B$ (LB and $\mathrm{Kn} 10)$, and $r p o S(\mathrm{Kn} 0.1$ and $\mathrm{Kn} 10)$ were observed. $d p p C$ is a gene involved in substrate transportation, but $r p o B$ and $r p o S$ are both involved in transcription, with $r p o S$ specifically involved transcription initiation. OLC969 also appeared to have an initial cost, though not necessarily as costly as in MG1655 (Figure 4.4). Nonetheless, mutations to $r b s R$ and $a r a A$, involved in transcription regulation and carbohydrate degradation, respectively, were found in all selection environments. However, the fact that these mutations were observed across conditions could indicate some level of general adaptation, or a strong advantage of those mutations for compensating the cost of pPB29 in this background. Mutations to rpoS were found in both MG1655 (Kn0.1 and Kn10) and OLC969 (Kn10). These may contribute to compensatory evolution for costs of plasmid carriage, given that both of these genotypes 
experienced some cost at the start of the experiment. Finally, PB1 and OLC969 shared missense mutations to undecaprenyl-phosphate alpha-N-acetylglucosaminyl 1-phosphate transferase (EC 2.7.8.33), which is involved in LPS O-antigen biosynthesis. 


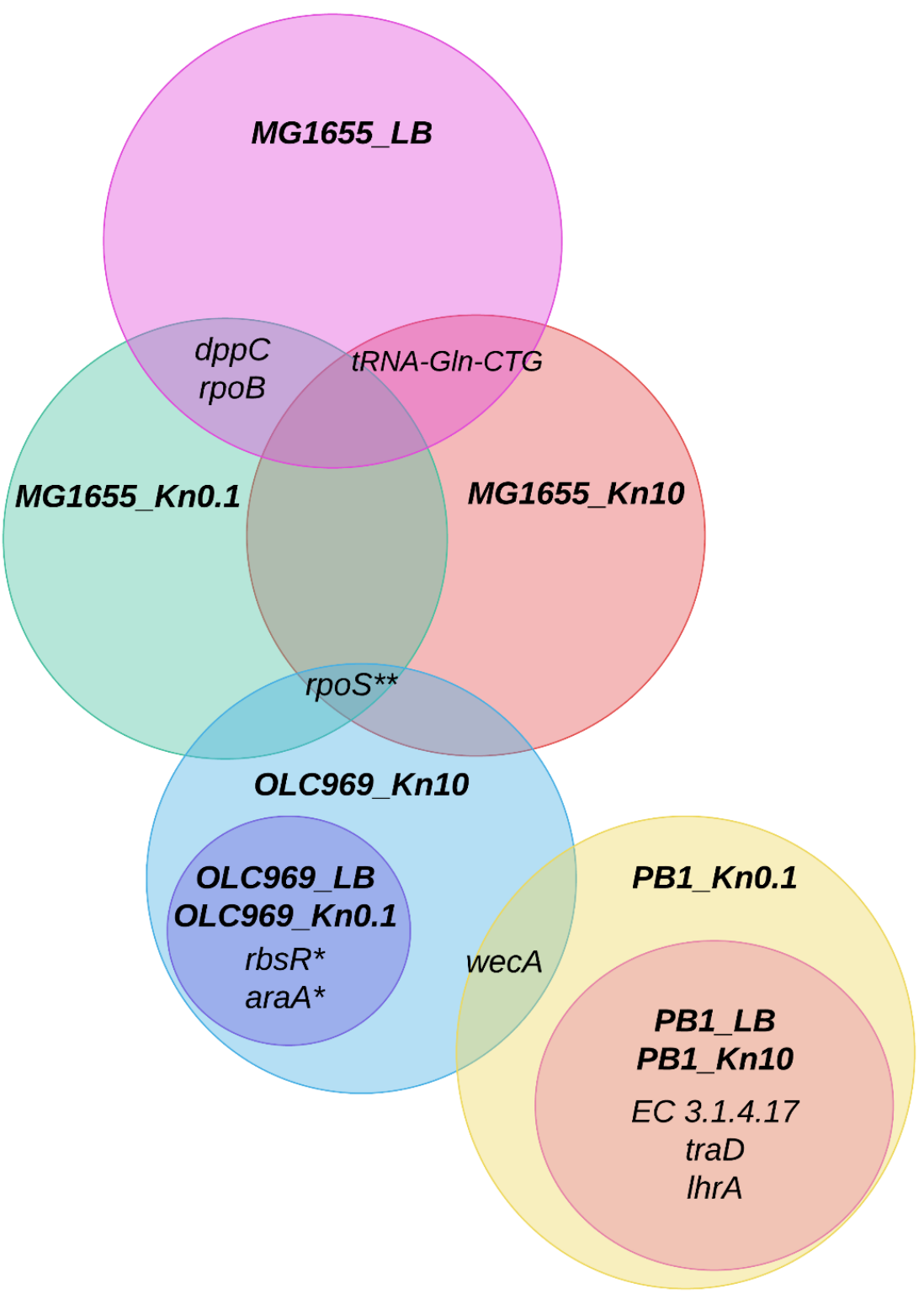

Figure 4.7: Mutations observed in multiple selection conditions (LB, Kn0.1 or Kn10) and genotypes (MG1655, PB1, and OLC969) following 500 generations of experimental evolution. Genes with $(*)$ represent mutations identical at the gene level; (**) signifies that the same mutation at the nucleotide level was observed within MG1655 but a different one was observed in OLC969. For the remaining mutations, distinct mutations were found in the same gene. 
Finally, I calculated the Jaccard index $(\mathrm{J})$ to quantify parallel evolution at the gene level between replicates in the three selection conditions for each genotype (Figure 4.8). This index relates the number of shared mutations between two points (such as between replicates, conditions, or genotypes) to the total number of mutations observed in both of those cases and can span from 0 (no overlap) to 1 (complete overlap). When comparing the selection conditions within each genotype, there is overlap between the genes observed in the three selection environments, however, there is more overlap for PB1 and OLC969, as denoted by the lighter grey in the heat map. With the exception of a few cases, namely OLC969 evolved in Kn10 compared to MG1655 evolved in Kn0.1 and Kn10 and PB1 evolved in Kn0.1 compared to OLC969 evolved in Kn10, there is no overlap in the genes observed across genotypes (Figure 4.8). This indicates that there is evidence of parallel evolution between the populations of each genotype evolved in the three conditions, but maintenance of the divergent evolution between strains was evident because the lack of convergence in mutations. 


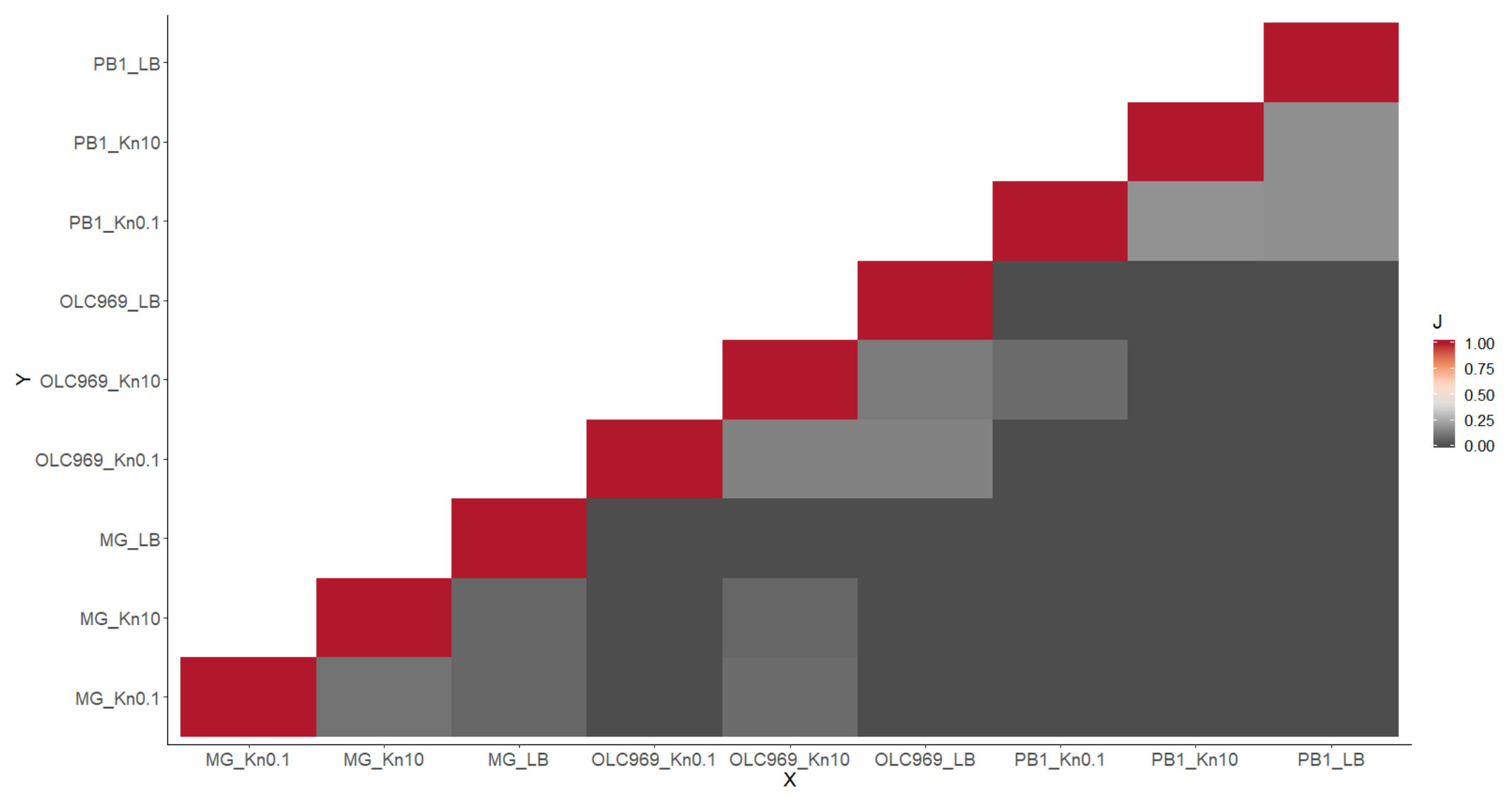

Figure 4.8: Jaccard Index ( $\mathrm{J}$ ) for gene-level overlap between selection conditions for each genotype. $\mathrm{J}$ was calculated by averaging the number of mutations over the three replicate populations, and then dividing the number of overlapping genes over the total number of mutations between those two conditions. Dark grey squares represent no overlap $(\mathrm{J}=0)$, where lighter grey squares represent increasing gene overlap. There was more overlap between the three selection conditions within a genotype, but few instances of overlap between genotypes. 


\subsection{Discussion}

Given the importance of plasmids in the dissemination of AMR, understanding all aspects of their maintenance over time is essential. Here, I have evolved three strains of E. coli with the MDR-plasmid pPB29 over 500 generations in three selection conditions. I measured changes in fitness and investigated the effect of selection on the prevalence of parallel genomic evolution.

I have attempted to address three major questions with these experiments: 1) how long will a plasmid persist, 2) does the level of selection help to stabilize the plasmid and 3) what is the likelihood of parallel evolution and how does the level of selection for the plasmid influence parallel evolution? To answer the first question, I had picked sixteen random colonies per replicate from each evolved population and streaked these on agar plates containing either kanamycin or ampicillin (Table 4.2). Loss was not common over the time course I measured, which indicates that pPB29 can persist for at least 500 generations. The level of plasmid selection by using various amounts of kanamycin did not seem to impact the overall outcome either; plasmid loss was slightly more common in the LB and Kn10 selection environments, but ultimately, the lack of kanamycin selection (i.e., LB only) did not result in more plasmid loss than those conditions with kanamycin. In terms of parallel evolution, I found many overlaps in the genes that had mutations following 500 generations, as there were several non-zero Jaccard Index scores when comparing within selection conditions, as well as between selection conditions for each genotype (Figures 4.8 and 4.9). However, this overlap was only prevalent when comparing selection conditions within a genotype, and overlap was not common between genotypes. Between MG1655, PB1, and OLC969 there was also some degree of 
parallelism, as there was overlap between the mutations observed between genotypes, but the $\mathrm{J}$ scores were low in most instances (Figure 4.9D).

In these experiments, I found that fitness generally increased over 500 generations, and that both the selection and competition environments can influence the fitness outcome to some degree (Figures 4.1-4.4). I found that the fitness of the evolved populations in the LB-only competitions (Figure 4.1) were generally positive and did not vary between selection condition. Fitness generally increased from 0 to 500 generations for competitions carried out in the LB and $1 \mathrm{X}$ ancestral MIC kanamycin conditions but did not change significantly over time for the $10 \mathrm{X}$ ancestral MIC competitions (Figures 4.2, 4.3, and 4.4). Selection condition, that is, whether the populations were passaged in $\mathrm{LB}, 0.1 \mu \mathrm{g} / \mathrm{ml}$, or $10 \mu \mathrm{g} / \mathrm{ml}$ kanamycin, did not contribute significantly to fitness in the different competitions overall. However, fitness was significantly different between populations selected in Kn0.1 and Kn10 for OLC969 in the LB competitions, and fitness between LB and Kn0.1 selected MG1655 were significantly different in 10X ancestral MIC competitions (Figure 4.4). This means that the strength of antibiotic selection (0-10 $\mu \mathrm{g} / \mathrm{ml}$ kanamycin) over 500 generations does have some impact on fitness when measured in varied concentrations of antibiotic following the evolution experiment.

As I mention above, there may be some discrepancies in fitness values reported for Figure 4.2, 4.3, and 4.4, as I used a different competitor for those experiments than I did in the LB-only competitions summarized in Figure 4.1. Additionally, PB1 showed a strange decrease in fitness across competition conditions (Figures 4.2B, 4.3B, and 4.4B), but an increase in Figure 4.1B; these LB competitions surprisingly contradict each other, despite the competition conditions being the same except for the use of a different 
competitor. Even if fitness changed as a result of using the different competitor, the general trend should have stayed the same, as I observed for MG1655 in all cases. Nonetheless, I also found that there was some level of parallel evolution which occurred, both within and between selection environments, as well as some level of parallelism between genotypes (Figures 4.8 and 4.9).

Harrison et al (2015), studying pQBR103 in P. fluorescens after 450 generations of selection in varied concentrations of mercury, found that fitness increased, and parallel evolution was common regardless of the selection condition. This was similar to my results, in that fitness did generally increase, at least for competitions in LB and $1 \mathrm{X}$ ancestral MIC kanamycin (Figures 4.1, 4.2, and 4.3), and most populations reached similar fitness. However, Harrison and colleagues only used a single genetic background in their study. My work here extends our understanding of plasmid-host coevolution by exploring the impact that different selection environments and time have on coevolution when the same plasmid evolves in different genetic backgrounds. This is important to consider, as I show that fitness associated with the same plasmid can follow different trajectories when in different hosts.

I found that plasmid maintenance is possible for extended periods of time and does not always require selection. It is evident that the plasmid was readily maintained for 500 generations, regardless of the initial costs of plasmid carriage. Persistence occurred regardless of the selection environment that the populations were evolved in (Table 4.2). Again, Dahlberg and Chao (2003) have found that a plasmid could persist for up to 1100 generations. However, theory suggests that costly plasmids will be lost over time due to purifying selection, so in the absence of other mechanisms, I would have 
expected pPB29 to have at least been lost in MG1655, where the initial cost of the plasmid is highest (Bergstrom et al. 2000; Harrison and Brockhurst 2012). Indeed, for cases when the plasmid began as a costly entity (i.e., MG1655), compensatory evolution appears to be a way for the plasmid to be maintained, as is likely to be the case here.

Selection can also have an impact on maintenance, stabilizing the plasmid and allowing for more time for compensatory evolution to occur, so loss in the selective environments was not anticipated to be as common as it might have been in LB-only selection, if it occurred at all (Harrison and Brockhurst 2012). However, as I reported in Chapter 3, conjugation can play an important role in plasmid maintenance within populations when there are competitors who are more fit than the plasmid-bearer. Again, this aligns with theory, as costly plasmids can be maintained by "hitchhiking" to fitter strains in the population, as described by Bergstrom and colleagues (Bergstrom et al. 2000). It might be useful to repeat such an experiment, where the plasmid-bearer is evolved along with a fitter competitor, for longer than 100 generations, to see if conjugation will again be the solution to maintaining the plasmid versus compensatory evolution. However, a similar result would be expected in this case, given that the same strains were used here as were used in those previous experiments.

Beyond the broader systems (selection, compensatory evolution, and conjugation) in place to explain and improve plasmid maintenance, there are several other mechanisms which may help improve plasmid stability within populations. Again, costly plasmids are expected to be lost due to removal by purifying selection, so loss in the absence of any of these mechanisms would be expected (Bergstrom et al. 2000; Harrison and Brockhurst 2012). Here, I used plasmid pPB 29 , which is $\sim 85 \mathrm{kbp}$, conjugative, and has genes related 
to stability, partitioning, a toxin-antitoxin system, and several AMR determinants. Based on read depth for reads associated with the plasmid relative to the reads associated with chromosomal DNA, pPB29 exists at a low-copy number (between $\sim 1-4$ copies) in these three genotypes. Many of these features can help stabilize a plasmid within a cell. Copy number, which frequently correlates with plasmid size, is important for maintenance in the face of segregational loss. For instance, a smaller, high copy number plasmid is expected to segregate between daughter cells during cell division simply through chance, as at least one of the many copies will end up in either daughter cell (Nordstrom and Austin 1989; Million-Weaver and Camps 2014). Larger plasmids with partitioning systems have a chromosome-like partitioning system, which also helps with dimer resolution following plasmid replication, to ensure the maintenance of the plasmid in progeny during cell division. This should decrease the chance of loss by accident and the subsequent competition in the population with the plasmid-free segregant which would presumably be more fit (Crozat et al. 2010; Dmowski and Jagura-Burdzy 2013). Postsegregational killing systems are also useful systems to maintain plasmids; a stable toxin and unstable antitoxin is encoded from the plasmid - the toxin can also be chromosomally encoded at times - and if the plasmid is lost, the toxin is able to remain for longer and kill plasmid-free cells when the unstable antitoxin is lost (Dmowski and Jagura-Burdzy 2013; Hernández-Arriaga et al. 2014). Additionally, resistance to antibiotics, such as kanamycin, indicates that plasmid loss should be quite rare, particularly when drug selection is used. This correlates with my data, as plasmid loss was infrequent in the absence of drug. These systems, usually in concert, will help to maintain the larger, low-copy plasmids, such as the one studied here. 
As discussed above, parallel evolution occurs when the same trait arises independently in multiple lineages, and parallel evolution can be observed on a hierarchy ranging from phenotypic parallel evolution to nucleotide-level parallel evolution (Schluter et al. 2004; Woods et al. 2006; Crozat et al. 2010; Bailey et al. 2015). Parallel evolution is thought to be indicative of adaptation, although there are other potential explanations, such as mutation rate variation that leads to observing mutations in certain regions more often, and mutation supply, rather than starting fitness, is an important driver of parallel evolution (Bailey et al. 2015). Theory indicates that parallel evolution is more likely when there is strong selection and much genetic variation. Further, parallel evolution increases as a population gets closer to fitness optimum, as fewer options for adaptation remain for the organism as it gets closer to a fitness peak. Some of the factors thought to influence parallel evolution, such as selection environment, have been assessed with respect to systems with and without plasmids (Bailey et al. 2015; Harrison et al. 2015).

Here, I found parallel evolution on several levels. Firstly, at the top of the parallel evolution hierarchy, is fitness. Here, fitness was not found to be significantly different between selection conditions overall, and many replicates reached the same fitness following 500 generations, however, there were differences between genotype (Figure 4.1). I note, however, that MG1655 and PB1 were, with a few exceptions, significantly more fit than their T0 ancestors. By contrast, for OLC 969 most end-point populations were not significantly more or less fit than their $\mathrm{T} 0$ counterparts, but there is parallelism within genotype in terms of fitness (Figure 4.1). 
At the next level observable from my data, the process level, there were several instances of parallelism. Transcription regulation was a common theme amongst mutations detected in the genomic data. Plasmid expression dysregulation is one possible source of plasmid costliness, so rapidly finding ways to compensate for this dysregulation, such as through the alteration of transcription, may be necessary for reducing costs (San Millan and MacLean 2017). Finally, at the gene level, I saw some evidence of parallelism (Figure 4.7). Six of the nine genes found to have overlap between replicates and conditions showed different mutations to the same genes. These include $d p p C, \operatorname{rpoB}$, tRNA-Gln-CTG, EC 3.1.4.17, $\operatorname{traD}$, and $\operatorname{lhr} A$; in cases where these genes appeared in different replicates or selection conditions, the site(s) of the mutations were different. As demonstrated in Figures 4.8 and 4.9, there were several non-zero Jaccard Index scores, indicating there was overlap in the genes which had mutations within and between selection conditions. The remaining three genes, $r b s R$, araA, and $r p o S$, had nucleotide-level parallelism, where the same change was present at the same position, indicating that I have found instances of parallel evolution across all levels that I was able to assess.

Mutation rate can vary across a genome, and when this occurs, it makes it more likely that certain mutations may arise repeatedly (Bailey et al. 2015). Presumably, genes that are mutated as a general response to the medium should occur more frequently and across all conditions, and there were not many instances of this. While I might not be able to directly assess the mutations which are a result of general adaptation to the LB environment in my experiment, I can compare the mutations that I found to those found by Wong and Seguin (2015). They selected for ciprofloxacin resistance in MG1655 with 
no plasmid in LB, and show which mutations resulted over the course of the experiment which might be associated with adaptation to the medium; none of these mutations overlap with those I found. This lack of overlap indicates that most of the mutations for MG1655 evolved with pPB29 might be in response to the plasmid, and not just as a response to evolving in the medium. Had there been a lot of overlap between the genes presented in Wong and Seguin (2015) with the mutations presented here, this would suggest that those mutations were likely not related to plasmid-carriage and would not necessarily warrant further investigation into their potential role in ameliorating the fitness costs of pPB29. However, while these results may be useful for assessing the mutations observed in MG1655, they do not necessarily represent what may have occurred in PB1 and OLC969, but it can inform the kinds of mutations which may have arisen as a response to growth for longer periods of time in LB.

Despite being able to conclusively state whether the mutations observed here are solely a response to evolving with a plasmid, my findings here are strengthened by the results from a similar study by Harrison et al (2015). They found that there were repeated mutations to a global regulatory system in response to the plasmid pQBR103 over time, which resulted in decreased transcription in the host. I found mutations to the genes encoding different parts of RNA polymerase $(r p o S, r p o B$, and $l h r A$ (a regulator of $r p o S)$ ), which may have a similar global effect, and help to regulate the costs of a plasmid, particularly when the cost results from the demand of maintaining the plasmid on the host. It has been found that there are certain pathways, like those related to metabolic and regulatory paths, in which mutations have been frequently observed in long-term evolution experiments with both bacteria and yeast (Crozat et al. 2010). Again, this lends 
support that the types of mutations observed here are an adaptive response, since genes related to transcription regulation were repeatedly found.

Bailey et al (2015) and Harrison et al (2015) found that parallel evolution was more common within replicates selected in the same environment, or even across selection conditions with varying levels of mercury, respectively. My results more closely follow those found by Harrison and colleagues, where I noticed that parallel evolution was more common between selection conditions though the various selection environments only varied by amount of drug (none to $10 \mu \mathrm{g} / \mathrm{ml}$ kanamycin), rather than completely different environments. I calculated the Jaccard Index for the mutations I observed across selection condition and genotype and found that there was a similar index amongst selection conditions, with smaller values calculated between genotypes (Figures 4.8 and 4.9). Nonetheless, parallel evolution did not occur to the same degree as they found, where mutations to the same sets of genes occurred across selection environments.

In conclusion, I find that pPB29 was retained over 500 generations, regardless of the level of selection. Fitness for MG1655 and PB1 generally increased significantly over 500 generations, but not for OLC969 in my initial LB experiments. However, in repeated LB competitions run alongside the $1 \mathrm{X}$ ancestral MIC and 10X ancestral MIC kanamycin competitions, fitness generally increased over 500 generations in the LB and $1 \mathrm{X}$ ancestral MIC competitions but stayed the same in the 10X ancestral MIC competitions. There were no significant differences in fitness overall between the selection conditions for each host-plasmid combination, and fitness deficits associated with plasmids can be overcome by evolution. Parallel evolution at the gene and nucleotide levels was observed 
and was frequently related to transcriptional genes, not only suggesting the adaptive role that these mutations might have, but also helping to point to the reason why the plasmid might exert a cost on its host in the first place. These results show us that plasmids can be maintained for a long time, and that similar strategies can be utilized by different hosts in response to the same plasmid. Lastly, it shows that different genotypes, despite starting fitness or selection condition, resulted in similar strategies for possible compensation, indicating that plasmid maintenance in populations might not be as simple as reducing selection or hoping that these strains will be outcompeted by more-fit, less-resistant competitors. These findings highlight the importance of developing strategies to stop plasmid transfer initially to reduce them in the population in the first place.

More specifically, I show that the fitness of the conjugative plasmid pPB29, while it may start out costly in its host, can evolve to be less of a burden on the host. However, despite selecting these host-plasmid combinations in three different selection conditions (LB, Kn0.1, and $\mathrm{Kn} 10$ ), providing selection pressure for the plasmid did not change the trajectory of fitness changes over 500 generations. This again highlights the importance that this plasmid can be maintained for at least 500 generations, which may not be useful for interventions such as restriction protocols. While many of the evolved populations for each genotype might have gained mutations related to transcriptional regulation, the genes themselves varied, showing that there are several ways that a plasmid might be maintained, regardless of the host it resides in. 


\section{Chapter 5: Gene $x$ Environment interactions in plasmid-carrying $E$. coli}

\subsection{Introduction}

Plasmid-mediated antimicrobial resistance (AMR) is a concerning problem. While AMR is certainly a threat to many areas of life, be it agriculture, environmental, or health, the threat to human health is extremely concerning. It has been estimated that upwards of 13700 Canadians could die per year as a direct result of AMR infections by 2050 if the prevalence of resistance is not reduced below the projected $40 \%$ (The Council of the Canadian Academies 2019). This threat to public health is increasingly worrisome, as many of our drugs of last resort are becoming ineffective against microbes harbouring resistance. Resistance to carbapenem antibiotics, a class of $\beta$-lactam drugs, is of particular concern, as the number of cases of carbapenemase-producing organisms (CPO) has increased five-fold between 2014 and 2017 in Canada (Public Health Agency of Canada 2018). Since genes encoding resistance to carbapenems are increasingly found on plasmids, and both mobilizable and conjugative plasmids can easily transfer within bacterial populations, understanding the costs of these plasmids and the factors that can serve to mediate plasmid spread and persistence is imperative (Bennett 2008; Naas et al. 2008; Pal et al. 2015; Conlan et al. 2019). Further research into these factors might help to adequately resolve questions about plasmid biology generally, and aid in the reduction of AMR more specifically.

Plasmids frequently impose a fitness deficit on their hosts in the absence of selection. This is thought to be due to any number of causes, but particularly from the energetic costs of maintaining the plasmid (Vogwill and MacLean 2015; San Millan and 
MacLean 2017). These plasmid-mediated costs can depend on gene-by-gene interactions between host and plasmid, whereby standing genetic variation can impact the fitness of the host-plasmid combination following plasmid acquisition (see Chapter 2 and Chapter 3 for a more rigorous explanation) (Vogwill and MacLean 2015). However, these are by no means the only types of interactions possible; indeed, gene-by-environment (GxE) interactions have also been shown to mediate variation in fitness, where the rank order in fitness for a collection of strains or mutants is not the same across various environments (Via and Lande 1985; Martin and Lenormand 2006; Hall 2013b). That is, a mutant might do rather poorly relative to other genotypes in one environment, but well in another. For example, Gifford et al (2016) found that for three environments, there was no significant change in the rank order of fitness of three rifampicin resistant mutants in Pseudomonas aeruginosa. Thus, while absolute fitness could vary in each environment, the fitness of strains relative to each other was preserved. However, a study by Hall (2013) showed that after adaptation of rifampicin-resistant E. coli in LB broth for 200 generations, gene-by-environment interactions are evident when strains are grown in other media. More recently, Clarke et al (2020) found that the fitness of six chromosomally-encoded drug resistant $E$. coli had variable fitness in ten environments, with rank order of fitness varying between environments. Taken together, this shows that there is evidence for environment having a potentially significant influence on the fitness of chromosomal AMR mutations (Hall 2013b; Clarke et al. 2020).

However, to date, it does not appear GxE has been systematically assessed for plasmids, and investigating such interactions might be useful for informing interventions which can help combat plasmid-mediated AMR, given the propensity for various 
mechanisms to preserve plasmids in populations (see Chapter 2 and Chapter $\mathbf{3}$ for a more in-depth analysis) (Bergstrom et al. 2000). That is, a broader understanding of GxE in plasmids, as well as finding out which environmental factors might make an organism less fit, may have several implications. Not only could this understanding allow us to better predict fitness outcomes generally, but it might be useful for designing interventions in wastewater treatment, for example, to reduce the further spread of AMR pathogens.

Determining if certain conditions can mediate plasmid fitness, particularly those conditions that are not considered "optimal", might allow a better understanding for why certain environments act as reservoirs for AMR bacteria, and of how plasmids persist in certain systems when they are otherwise considered less fit. Since bacteria can be found in virtually any environment, they may encounter a variety of growth conditions, which may or may not influence fitness. This in turn can impact the likelihood that the bacterium might survive and potentially lead to an AMR infection in an animal or human if it is able to move from one environment and to another. Because of this, and since animals and humans interact with each other and the wider environment, GxE may be an important factor in understanding the spread and maintenance of AMR pathogens, particularly when such pathogens can then cause disease.

All organisms have an ideal set of conditions in which they thrive, and variations to these conditions can impair the ability of that organism to survive. Escherichia coli, for instance, grows best at $37^{\circ} \mathrm{C}$, prefers neutral $\mathrm{pH}$, is able to use a variety of nutrient sources, and can grow in the presence or absence of oxygen, as it is a facultative anaerobe (Reinikainen et al. 1985; Unden and Trageser 1991). E. coli uses fermentation rather than 
respiration to produce ATP in the absence of oxygen without an alternative electron acceptor (such as nitrate), which produces much less energy than respiration (Unden and Trageser 1991; Gunsalus and Park 1994; Finn et al. 2017). Further, resource depletion can impair growth rates in E. coli, suggesting lower glucose levels, for example, can further impair the growth of already struggling competitors (Shehata and Marr 1971). Carbon sources, such as glucose, are important for metabolism, as these sugars are essential electron donors which are used for metabolism, and lower levels of glucose can impair growth or induce the use of alternate carbohydrate uptake and other metabolic pathways (Manché et al. 1999; Olvera et al. 2009; Martínez-Gómez et al. 2012). Finally, variation in $\mathrm{pH}$ from neutral can have numerous effects, from changing ionization states of amino acids and impacting protein structure and function, as well as interfering with proton gradients, such as those required for ATP production (Jin and Kirk 2018). Nonetheless, while the conditions mentioned above are the optimal conditions, there is frequently a range within these sets of conditions where an organism can still grow and survive.

Environment not only impacts the fitness of bacteria, but some factors, particularly $\mathrm{pH}$ and oxic vs anoxic growth, can have a direct effect on the level of resistance granted by resistance mutations or genes. For example, there is evidence that resistance to aminoglycoside antibiotics is increased at lower $\mathrm{pH}$, as well as in anoxic environments (Schlessinger 1988; DeMars et al. 2016). Such patterns of resistance are thought to result from the impact of $\mathrm{pH}$ and aerobic respiration on the electron transport chain (ETC), where alterations to these factors change the ETC, which in turn can directly influence the ability for drug to be imported into the cell (Schlessinger 1988; 
DeMars et al. 2016). Resistance to $\beta$-lactam drugs via $\beta$-lactamases can also be mediated by the environment, such that activity is decreased in lower $\mathrm{pH}$ and with anoxic growth; that is, bacteria with $\beta$-lactamase resistance, such as carbapenemase-producing organisms, tend to be more resistant at higher $\mathrm{pH}$ and during oxic growth (DeMars et al. 2016).

However, not only does environment affect drug entry into the cell, but it can also impact drug chemistry. Aminoglycoside drugs, for instance, have a net-positive charge, so variation in $\mathrm{pH}$ can interfere with drug association with the membrane and the ability to utilize the electrical potential difference to cross the membrane, while $\beta$-lactam drugs benefit from protonation to activate the drug (Blaser and Lüthy 1988; Delcour 2009). While such trends are likely to be observed for plasmid-mediated resistance in the same way as their chromosomal counterparts due to shared mechanisms, explicitly testing these relationships will provide confirmation (Bennett 2008; Blair et al. 2015). Particularly with strains carrying carbapenemase-encoding plasmids, determining which environments can increase drug susceptibility would be enticing for further investigating ways to combat these strains.

As such, in this study I assess the impact of varying $\mathrm{pH}$, oxygen level, and nutrient availability on the fitness of six plasmid-bearing strains of $E$. coli. Three of the plasmids investigated here confer resistance to carbapenem antibiotics, meaning that investigating potential GxE interactions with these plasmids can be extremely useful, not only for understanding GxE in plasmids generally, but the costs and GxE interactions involved in carbapenem-resistance plasmids specifically. I hypothesize that I will observe GxE interactions, and that these will be significant contributors to fitness. More 
specifically, I predict that certain factors, like reduced glucose availability, non-neutral $\mathrm{pH}$, or the absence of oxygen will exert increased effects on fitness, as the transconjugants will not only experience the cost related to the plasmid but will also then be faced with further stresses. Head-to-head competitions will be used to measure fitness between each strain as compared to their plasmid-free ancestor in twelve environmental combinations differing by the presence or absence of oxygen, regular carbon or low carbon availability, and $\mathrm{pH} 6,7$, and 8 . Additionally, I will assess changes in resistance across these environments, and investigate potential mechanisms for costs.

\subsection{Materials and Methods}

\subsubsection{Strain and General Culturing Information}

Escherichia coli (E. coli, MG1655, ST-37 (PB4), and ST-315(PB29)) (Basra et al. 2018) and Klebsiella oxytoca (Cooper et al. n.d.) were streaked on Lysogeny Broth (LB; $1 \%$ tryptone, $0.5 \%$ yeast extract, $1 \% \mathrm{NaCl}$ ) plates (LB with $1.5 \%$ agar) and were incubated inverted overnight $(\sim 20-24$ hours $)$ at $37^{\circ} \mathrm{C}$. PB29 and the Klebsiella isolates were used as the plasmid donors. Species and source information, as well as other pertinent strain information is also summarized (Table 5.1). Deletion of the lac operon was done using homologous recombination using a plasmid to make MG1655 $\mathrm{lac}$ and PB4Alac, as described in Chapter 3. Transconjugant strains were selected for on LB with $15 \%$ agar with the presence of the selective antibiotic (either $100 \mu \mathrm{g} / \mathrm{ml}$ kanamycin for pPB29 or $1.5 \mu \mathrm{g} / \mathrm{ml}$ meropenem for KPC plasmids). Dilutions were carried out using $1 \mathrm{X}$ M9 Salt Solution diluted from 10X M9 $\left(\mathrm{Na}_{2} \mathrm{HPO}_{4}-2 \mathrm{H}_{2} \mathrm{O}\right.$ at $37.6 \mathrm{~g} / \mathrm{L}, \mathrm{KH}_{2} \mathrm{PO}_{4}$ at $15 \mathrm{~g} / \mathrm{L}$, 
and $\mathrm{NaCl}_{2}$ and $\mathrm{NH}_{4} \mathrm{Cl}$ at $2.5 \mathrm{~g} / \mathrm{L}$ each and autoclaved for 15 minutes at $121^{\circ} \mathrm{C}$ ). All plates were incubated inverted overnight at $37^{\circ} \mathrm{C}$ wrapped in Parafilm. Overnight cultures of $E$. coli were grown in LB broth for 24 hours at $37^{\circ} \mathrm{C}$, either with or without the presence of antibiotics as required, unless otherwise stated. Kanamycin $\left(50 \mathrm{mg} / \mathrm{ml}\right.$, diluent $\left.\mathrm{dH}_{2} \mathrm{O}\right)$ and meropenem $\left(10 \mathrm{mg} / \mathrm{ml}\right.$, diluent $\left.\mathrm{dH}_{2} \mathrm{O}\right)$ stocks were prepared, and filter sterilized as required and stored at $-20^{\circ} \mathrm{C}$.

Table 5.1: Serotype, source of isolate, and plasmid information for the E. coli and $K$. oxytoca strains used in this experiment. Recipients are the hosts used for assessing GxE, whereas the ETC mutants were used for assessing the impact that the ETC has on patterns in resistance to the aminoglycoside drug kanamycin. Donor strains were used for transferring plasmids into the two plasmid recipients.

\begin{tabular}{|c|c|c|c|c|c|c|}
\hline Strain & Species & Serotype & Source & $\begin{array}{l}\text { Known } \\
\text { Plasmids }\end{array}$ & $\begin{array}{c}\text { ETC } \\
\text { Mutation }\end{array}$ & Reference \\
\hline \multicolumn{7}{|l|}{ Recipients } \\
\hline MG1655 $\Delta l a c$ & $\begin{array}{l}\text { Escherichia } \\
\text { coli }\end{array}$ & - & - & None & & $\begin{array}{c}\text { (Bachmann 1972; Guyer et } \\
\text { al. 1981) }\end{array}$ \\
\hline PB4 $4 l a c$ & $\begin{array}{l}\text { Escherichia } \\
\text { coli }\end{array}$ & ST-1670 & UTI & None & & Basra et al. 2018 \\
\hline \multicolumn{7}{|l|}{ ETC Mutants } \\
\hline Kn14 & $\begin{array}{l}\text { Escherichia } \\
\quad \text { coli }\end{array}$ & - & - & None & $\begin{array}{l}\text { cyoA } \\
\text { W82* }\end{array}$ & \\
\hline $\mathrm{Kn} 15$ & $\begin{array}{l}\text { Escherichia } \\
\text { coli }\end{array}$ & - & - & None & $\begin{array}{c}u b i F \\
\text { Q120* }\end{array}$ & \\
\hline Gn12 & $\begin{array}{l}\text { Escherichia } \\
\text { coli }\end{array}$ & - & - & None & $\begin{array}{l}\text { cyoA } \mathrm{I} 127 \\
\text { frameshift }\end{array}$ & \\
\hline Gn14 & $\begin{array}{l}\text { Escherichia } \\
\text { coli }\end{array}$ & - & - & None & $\begin{array}{c}u b i F \\
\mathrm{D} 342 \mathrm{H}\end{array}$ & \\
\hline \multicolumn{7}{|l|}{ Donors } \\
\hline PB29 & $\begin{array}{l}\text { Escherichia } \\
\text { coli }\end{array}$ & ST-315 & $\begin{array}{l}\text { Blood } \\
\text { infection }\end{array}$ & IncFII & & Basra et al. 2018 \\
\hline $\mathrm{C} 12$ & $\begin{array}{l}\text { Klebsiella } \\
\text { oxytoca }\end{array}$ & - & Wastewater & $\operatorname{IncFIB}(\mathrm{K})$ & & (Cooper et al. n.d.) \\
\hline $\mathrm{C} 16$ & $\begin{array}{l}\text { Klebsiella } \\
\text { oxytoca }\end{array}$ & - & Wastewater & $\operatorname{IncFIB}(\mathrm{K})$ & & (Cooper et al. n.d.) \\
\hline $\mathrm{C} 33$ & $\begin{array}{c}\text { Klebsiella } \\
\text { oxytoca }\end{array}$ & - & Wastewater & $\begin{array}{l}\operatorname{IncFIB(K),} \\
\operatorname{IncFIA(HI1)}\end{array}$ & & (Cooper et al. n.d.) \\
\hline
\end{tabular}




\subsubsection{Conjugation Protocol}

As described in Chapter 3, I used a plasmid from a blood-infection isolate of $E$. coli ST-315 strain PB29, which I will continue to refer to as pPB29 (Basra et al. 2018). pPB29 belongs to the IncFII family of plasmids, is conjugative, and kept at a low copy number ( $\sim 4$ copies per cell). This plasmid is known to carry the following resistance genes: $\operatorname{aac}(2)-\mathrm{IIa}, \operatorname{tet}(\mathrm{A}), b_{\mathrm{CTX}-\mathrm{M}-15}, b_{a_{\mathrm{OXA}-1}}$. I also used plasmids from three strains of Klebsiella oxytoca, C12, C16, and C33; I will refer to the plasmid from each of these isolates as pC12, pC16, and pC33, respectively. C12 and C16 both had IncFIB(K) plasmids, which carry the following resistance genes: bla KPC-2, bla $_{\mathrm{OXY}-6-2,}$ sul1, aadA11, aac(6')-Ib3, and $a a c\left(6^{\prime}\right)-\mathrm{Ib}-\mathrm{cr}$. C33 also carried an $\operatorname{IncFIB}(\mathrm{K})$ plasmid with the same genes, and an IncFIA(HI1) plasmid with bla

Plasmids were transferred to their hosts by conjugation. Overnight cultures were diluted 1:10 the following day, grown to early log phase, and then mixed in a 1:10 ratio of plasmid donor to recipient. Mating mixtures were then either spotted on LB plates and incubated at $37^{\circ} \mathrm{C}$ overnight, or liquid mixtures were kept at $37^{\circ} \mathrm{C}$ overnight without shaking. The following day, a portion of the spots on LB plates were resuspended in 1 $\mathrm{mL}$ of LB, diluted, and plated on selective media; liquid mating mixtures were diluted to $10^{-4}$ and plated. Plates were checked the following day for transconjugants, with donor cells appearing blue on the X-gal+IPTG plates, and the Lac ${ }^{-}$recipient appearing white and resistant to the drug. Transconjugants from matings between the host strains and the donor PB29 were selected on LB plates supplemented with X-gal, IPTG, and kanamycin and transconjugants from matings with $K$. oxytoca $\mathrm{C} 12, \mathrm{C} 16$, and $\mathrm{C} 33$ were selected on LB plates with X-gal, IPTG, and meropenem. 


\subsubsection{Media and Environment Set-Up}

pH and Glucose Conditions

Minimal medium at $\mathrm{pH} 6,7$, and 8 were made using a modified M9+glucose medium with phosphate buffer. A volume of the appropriate ratio of $0.2 \mathrm{M}$ dibasic sodium phosphate and $0.2 \mathrm{M}$ monobasic sodium phosphate were mixed to get the appropriate $\mathrm{pH}$ (Table 2). This volume was mixed 1:1 with the appropriate volume of water and 5X M9 salts to obtain a 1X M9 salts concentration. The mixture was autoclaved for 20 minutes at $121^{\circ} \mathrm{C}$, and once cooled, sterile $1 \mathrm{M} \mathrm{MgSO}_{4}$ and $1 \mathrm{M} \mathrm{CaCl}_{2}$ were added to a final concentration of $2 \mathrm{mM}$ and $0.1 \mathrm{mM}$, respectively. Following this, $1.1 \mathrm{M}$ glucose was added to the "regular" and "low" glucose condition medium for a final concentration of $22.2 \mathrm{mM}$ and $11.1 \mathrm{mM}$, respectively.

Table 5.2: Preparation of $50 \mathrm{ml}$ of $0.1 \mathrm{M}$ phosphate buffer to be mixed with $50 \mathrm{ml}$ of appropriate volume of 5X M9 salts and water.

\begin{tabular}{ccc}
\hline $\mathrm{pH}\left(25^{\circ} \mathrm{C}\right)$ & $\begin{array}{c}\text { Volume } 0.2 \mathrm{M} \text { dibasic sodium } \\
\text { phosphate }(\mathrm{mL})\end{array}$ & $\begin{array}{c}\text { Volume } 0.2 \mathrm{M} \text { monobasic sodium } \\
\text { phosphate }(\mathrm{mL})\end{array}$ \\
\hline \hline 6.0 & 6.25 & 43.75 \\
7.0 & 30.5 & 19.5 \\
8.0 & 47.35 & 2.65 \\
\hline
\end{tabular}

\section{Oxic and Anoxic Conditions}

96-well plates in the oxic condition were grown at $37^{\circ} \mathrm{C}$ with shaking in a tabletop shaker at 150RPM. Plates were sealed around the edges with Parafilm. Plates grown in anoxic conditions were grown in a COY Vinyl Anaerobic Chamber at $0 \mathrm{PPM} \mathrm{O}_{2}$ 
and 2.0PPM $\mathrm{H}_{2}$ with nitrogen as the background gas. Anoxic plates were grown at $37^{\circ} \mathrm{C}$ with the absence of shaking.

\subsubsection{Measurement of Relative Fitness}

Fitness was measured using head-to-head competition assays in mixed culture for the six transformant strains obtained. Overnight cultures of each transformant and plasmid-free ancestor were inoculated in $200 \mathrm{ul}$ each of the appropriate medium in quadruplicate in 96-well microwell plates. The following day, each plasmid-host combination, and their respective $\mathrm{Lac}^{+}$ancestor, were diluted 1:200 in $200 \mathrm{ul}$ of the appropriate medium in new 96-well plates, for a total dilution of 1:100, representing a 1:1 ratio of transconjugant to ancestor. For $\mathrm{PB} 4 \Delta l a c+\mathrm{pC} 12$ host/plasmid combination, a ratio of 3:1 was used for the transconjugant to ancestor, as the density of PB4 $\Delta l a c+\mathrm{pC} 12$ was so low as to produce very few colonies at the start of a competition when mixed in equal volumes with its ancestor and were not observed after 24 hours in many cases. Initial (T0) cultures were mixed and frozen with a final concentration of $25 \% \mathrm{v} / \mathrm{v}$ glycerol and stored at $-80^{\circ} \mathrm{C}$. Competition plates were sealed with Parafilm and incubated at $37^{\circ} \mathrm{C}$ in the presence or absence of oxygen for 24 hours. Following this period, a glycerol stock of the T24 cultures were made as above and frozen at $-80^{\circ} \mathrm{C}$. Relative counts for each plasmid-free ancestor and transformant pair was completed using a ten-fold dilution series from the frozen stocks in 1X M9 at both time points and plating 25ul of the appropriate dilutions on $\mathrm{LBA}+\mathrm{X}-\mathrm{Gal}+\mathrm{IPTG}$ plates. Following overnight growth at $37^{\circ} \mathrm{C}$, the number of transformant and plasmid-free ancestors were counted, and these counts 
were used to calculate relative fitness using the following calculation derived by

Dykhuizen and Hartl (1983):

$$
\text { Relative Fitness }=1+\frac{\ln \ln \left(\frac{\text { T24transconjugant }}{\text { T0transconjugant }}\right)-\ln \ln \left(\frac{T 24 \text { ancestral }}{\text { T0ancestral }}\right)}{\text { number of generations }}
$$

\subsubsection{Nitrate-supplemented competitions}

A set of competitions was completed as above with the addition of $10 \mathrm{mM}$ potassium nitrate to the $\mathrm{pH} 6.0$ with low glucose medium for $\mathrm{PB} 4 \Delta \mathrm{lac}+\mathrm{pC} 33$ and $\mathrm{pH} 8.0$ with low glucose medium for MG1655 $\mathrm{lac}+\mathrm{pC} 12.10 \mathrm{mM}$ was chosen as a previous report suggested that concentrations $5 \mathrm{mM}$ and above was a sufficient amount of nitrate for E. coli to use for reduction in anoxic conditions (Tiso and Schechter 2015). Both the transconjugant and ancestor were acclimated to the medium with and without nitrate for 24 hours prior to beginning competitions. Competitions were run in triplicate, with competitions run in the presence and absence of oxygen, with and without the nitrate supplement.

\subsubsection{Measurement of Susceptibility}

All minimum inhibitory concentration (MIC) assays were done by serially diluting the antibiotic two-fold in the appropriate medium in a 96-well plate. Overnight cultures of the strains were diluted to 1:100 in the fresh medium with the antibiotic dilution series and incubated overnight. Plates were read at OD600 using a plate reader the following day. 


\subsubsection{Statistical Analysis}

Statistical analyses carried out using $R$ package (R Core Team, 2019) and RStudio (RStudio Team, 2018). Figures were produced using R package ggplot2 (Wickham, 2016). Interactions were analysed using a multi-factorial ANOVA with Fitness as the dependent variable and environments ( $\mathrm{pH}$, glucose, oxygen), plasmid, and host as the independent variables. Assumptions for homogeneity of variances were tested using Levene's test and comparisons for significant factors and interaction terms were analysed using a Tukey's post-hoc test.

\subsection{Results}

\subsubsection{Effect of Genetic Background}

Overall, the carbapenemase plasmids resulted in the highest costs, when compared to pPB29 (Figure 5.1). These values are the averaged fitness measures across all environments, so they consider the change in the face of each type of environment. $\mathrm{PB} 4 \Delta l a c+\mathrm{pPB} 29$ is equally as fit as its plasmid-free ancestor $(\mathrm{t}(47)=0.65254, \mathrm{p}=0.5172)$, while the remaining strains MG1655 $l a c+p P B 29(\mathrm{t}(47)=-2.2975, \mathrm{p}=0.026)$, MG1655 $l a c+\mathrm{pC} 12(\mathrm{t}(47)=-13.071, \mathrm{p}<0.01), \mathrm{PB} 4 \Delta l a c+\mathrm{pC} 16(\mathrm{t}(47)=-17.097, \mathrm{p}<0.01)$ and $\mathrm{PB} 4 \Delta l a c+\mathrm{pC} 33(\mathrm{t}(47)=-11.582, \mathrm{p}<0.01)$, were significantly less fit than their ancestors overall. However, a one-way ANOVA and subsequent Tukey's HSD post-hoc test analysing the interaction between the strain (host-plasmid combination in this case) 


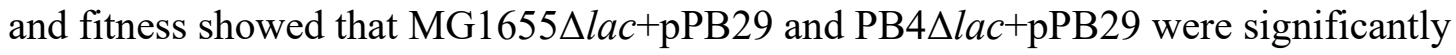
more fit than the remaining strains $[\mathrm{F}(5,282)=56.62, p=<0.001 ; \mathrm{p}$-adj $<0.001]$.

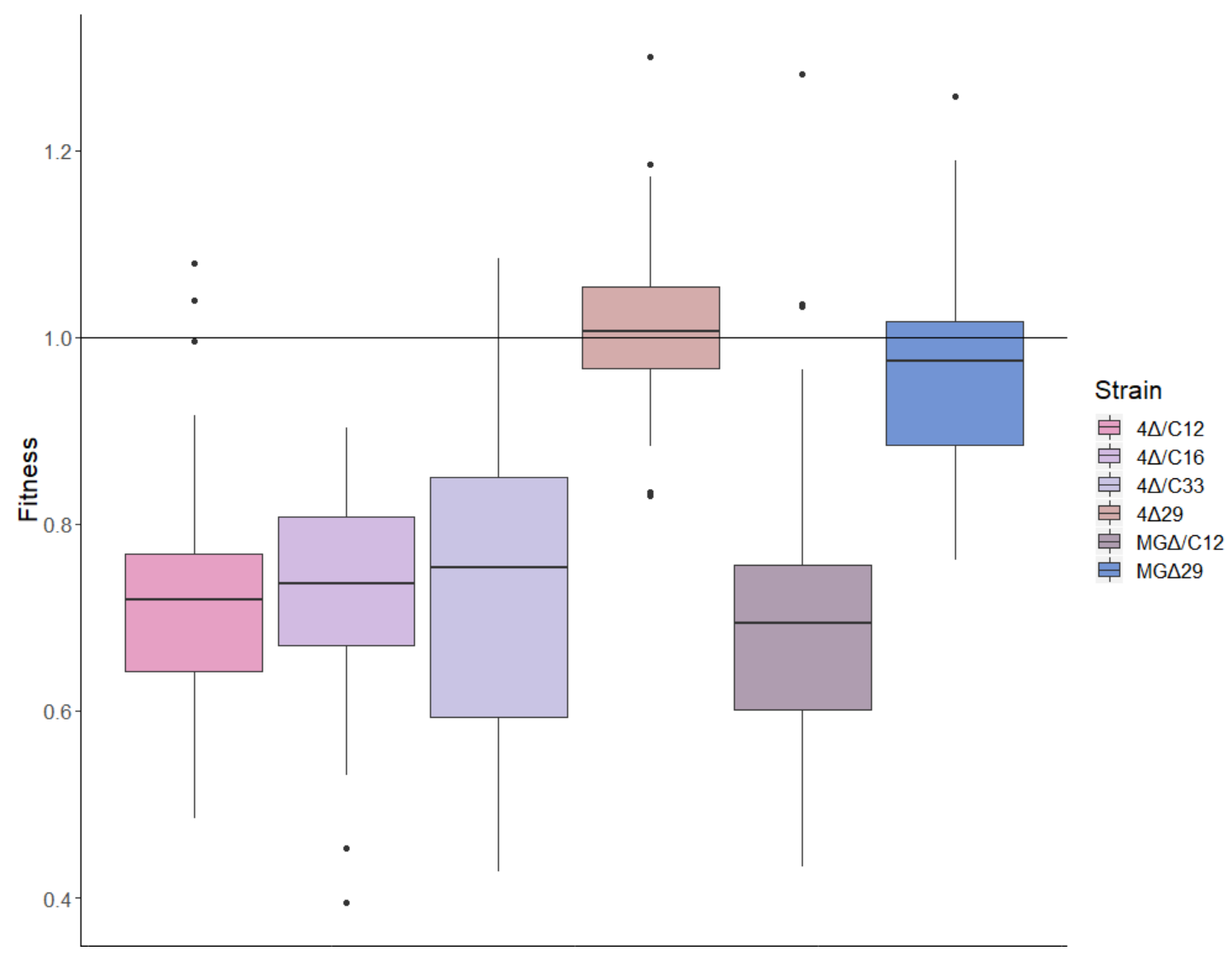

Figure 5.1: The fitness of six host-plasmid combinations averaged across twelve environments. Average relative fitness of MG1655 $l a c+\mathrm{pC} 16, \mathrm{MG1655 \Delta lac}+\mathrm{pPB} 29$, $\mathrm{PB} 4 \Delta l a c+\mathrm{pC} 12, \mathrm{~PB} 4 \Delta l a c+\mathrm{pC} 16, \mathrm{~PB} 4 \Delta l a c+\mathrm{pC} 33$, and PB4 $\Delta l a c+\mathrm{pPB} 29$ was measured against their respective plasmid-free ancestor in a head-to-head competition assay in modified M9+glucose medium with phosphate buffer. Fitness of MG1655 $l a c+p C 16$,

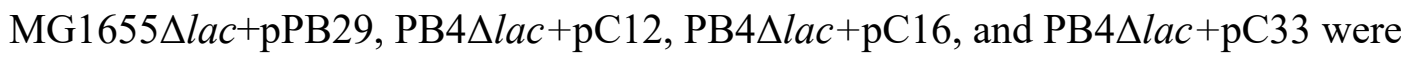
significantly less fit than their plasmid-free ancestors (t-test, $p>0.05$ ). Fitness of the pPB29 carriers were significantly more fit than those carrying pC12, pC16, and $\mathrm{pC} 33$ (One-way ANOVA with Tukey's post-hoc test, p-adj <0.05). 


\subsubsection{Effect of environmental factors on fitness}

\section{Effect of environmental factors on fitness}

Next, the effects of the environmental factors were assessed. Changes in $\mathrm{pH}$ were not found to have an overall effect on the fitness when considering all strains (Table 5.7). This is also apparent in Figure 5.2, as the average fitness for each strain at each $\mathrm{pH}$ is not much different than the others. However, when broken down by genotype, $\mathrm{pH}$ was a

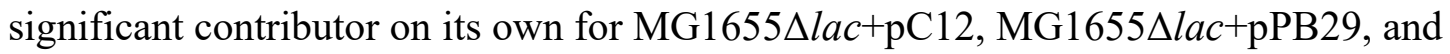
PB4 $l a c+\mathrm{pC} 33$ (Tables 5.3, 5.4, and 5.5). This can be observed more clearly in Figure 5.2, where fitness is higher at $\mathrm{pH} 6$ for MG1655 $l a c+\mathrm{pC} 12$ and MG1655 $l a c+\mathrm{pPB} 29$, and fitness is higher at $\mathrm{pH} 7$, but lower at $\mathrm{pH} 6$ and 8 for $\mathrm{PB} 4 \Delta l a c+\mathrm{pC} 33$. 


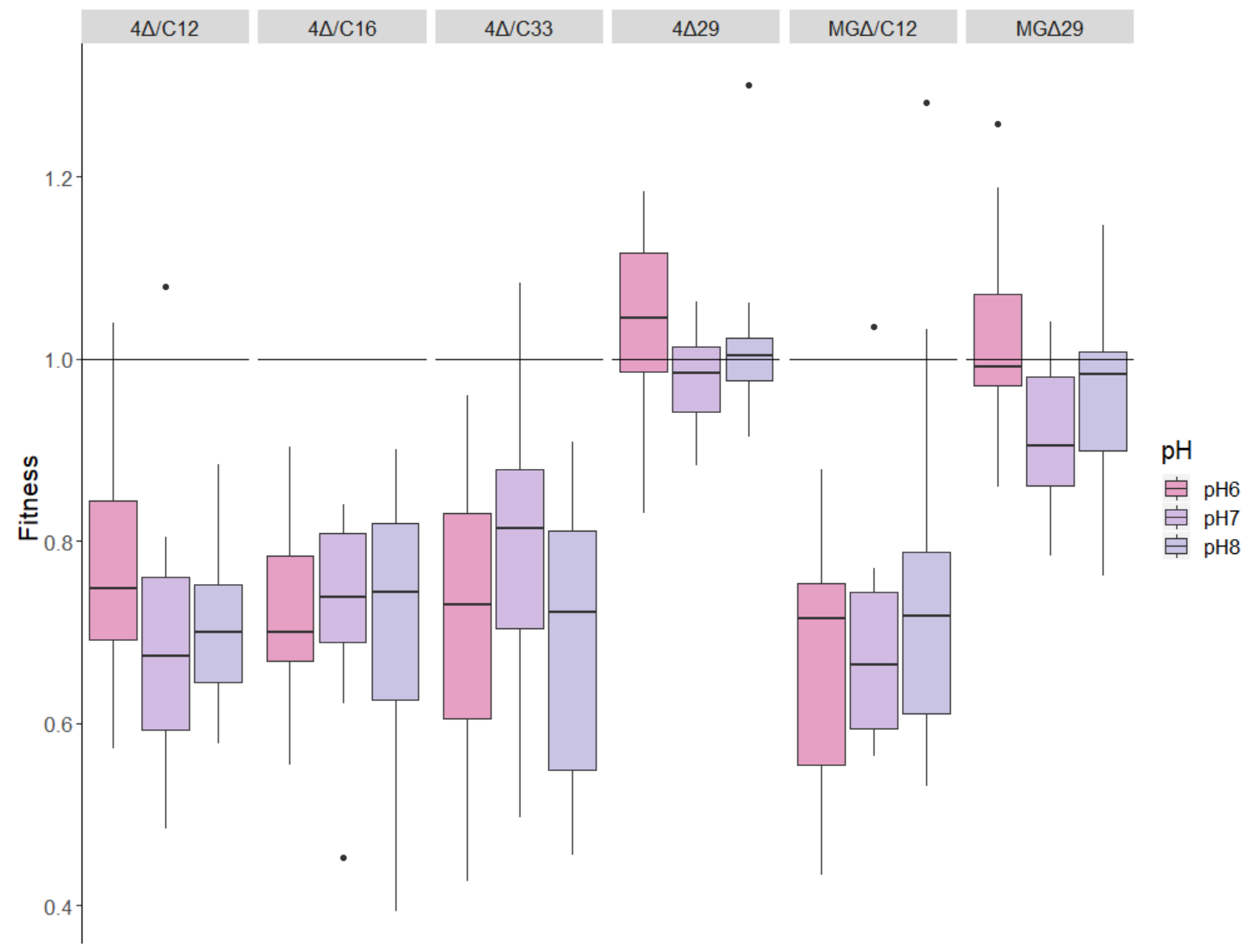

Figure 5.2: The average fitness of six host-plasmid combinations in media with $\mathrm{pH}$ 6.0, 7.0, and 8.0. Average relative fitness of MG1655 lac $+\mathrm{pC} 16, \mathrm{MG} 1655 \Delta l a c+\mathrm{pPB} 29$, $\mathrm{PB} 4 \Delta l a c+\mathrm{pC} 12, \mathrm{~PB} 4 \Delta l a c+\mathrm{pC} 16, \mathrm{~PB} 4 \Delta l a c+\mathrm{pC} 33$, and PB4 $l a c+\mathrm{pPB} 29$ was measured against their respective plasmid-free ancestor in a head-to-head competition assay in modified M9+glucose medium with phosphate buffer. $\mathrm{pH}$ was not a significant factor overall (5-way ANOVA of Fitness $\sim$ Host*Plasmid*Glucose*pH*Oxygen, $\mathrm{p}>0.05$ ), but

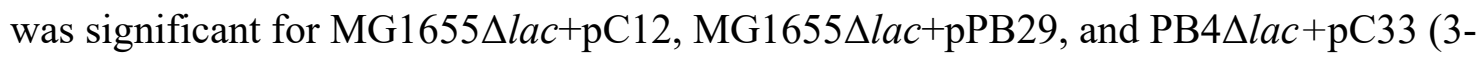
way ANOVA of Fitness $\sim$ Glucose*pH*Oxygen, $p<0.05$ ). 
Table 5.3: 3-way ANOVA for the effect of three environmental factors on fitness for MG1655 $l a c+\mathrm{pC} 12$.

\begin{tabular}{ccccccc}
\hline & Df & $\begin{array}{c}\text { Sum } \\
\text { Sq }\end{array}$ & $\begin{array}{c}\text { Mean } \\
\text { Sq }\end{array}$ & F value & $\operatorname{Pr}(>\mathrm{F})$ & Significance \\
\hline \hline Oxygen & 1 & 0.0039 & 0.00386 & 0.284 & 0.597428 & \\
pH & 2 & 0.2093 & 0.10464 & 7.706 & 0.001637 & $* *$ \\
Glucose & 1 & 0.2487 & 0.24871 & 18.316 & 0.000132 & $* * *$ \\
Oxygen:pH & 2 & 0.0167 & 0.00837 & 0.616 & 0.545596 & \\
Oxygen:Glucose & 1 & 0.0224 & 0.02244 & 1.652 & 0.206836 & \\
pH:Glucose & 2 & 0.2592 & 0.12959 & 9.544 & 0.000473 & $* * *$ \\
Oxygen:pH:Glucose & 2 & 0.1172 & 0.05859 & 4.315 & 0.020906 & $*$ \\
Residuals & 36 & 0.4888 & 0.01358 & & & \\
\hline
\end{tabular}

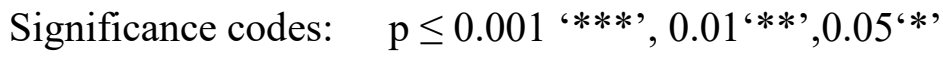

Table 5.4: 3-way ANOVA for the effect of three environmental factors on fitness for $\mathrm{PB} 4 \Delta l a c+\mathrm{pPB} 29$.

\begin{tabular}{ccccccc}
\hline & Df & Sum Sq & $\begin{array}{c}\text { Mean } \\
\text { Sq }\end{array}$ & F value & $\operatorname{Pr}(>F)$ & Significance \\
\hline \hline Oxygen & 1 & 0.00963 & 0.00963 & 2.133 & 0.1528 & \\
pH & 2 & 0.02848 & 0.01424 & 3.153 & 0.0547 & \\
Glucose & 1 & 0.00425 & 0.00425 & 0.942 & 0.3382 & \\
Oxygen:pH & 2 & 0.01768 & 0.00884 & 1.958 & 0.1559 & \\
Oxygen:Glucose & 1 & 0.00383 & 0.00383 & 0.848 & 0.3633 & \\
pH:Glucose & 2 & 0.02168 & 0.01084 & 2.399 & 0.1051 & \\
Oxygen:pH:Glucose & 2 & 0.12449 & 0.06225 & 13.782 & $3.60 \mathrm{E}-$ & $* * *$ \\
Residuals & 36 & 0.1626 & 0.00452 & & & \\
\hline
\end{tabular}

Significance codes: $\quad \mathrm{p} \leq 0.001^{\text {' } * * *}, 0.01^{\text {'**', }}, 0.05^{\text {‘* }}$, 
Table 5.5: 3-way ANOVA for the effect of three environmental factors on fitness for $\mathrm{PB} 4 \Delta l a c+\mathrm{pC} 33$.

\begin{tabular}{ccccccc}
\hline & Df & $\begin{array}{c}\text { Sum } \\
\text { Sq }\end{array}$ & $\begin{array}{c}\text { Mean } \\
\text { Sq }\end{array}$ & F value & $\operatorname{Pr}(>\mathrm{F})$ & Significance \\
\hline \hline Oxygen & 1 & 0 & 0.00003 & 0.003 & 0.953474 & \\
pH & 2 & 0.0965 & 0.04826 & 5.388 & 0.008978 & $* *$ \\
Glucose & 1 & 0.0083 & 0.00829 & 0.925 & 0.342547 & $* *$ \\
Oxygen:pH & 2 & 0.4627 & 0.23135 & 25.827 & $1.11 \mathrm{E}-07$ & $* * *$ \\
Oxygen:Glucose & 1 & 0.0063 & 0.00629 & 0.703 & 0.407433 & $* *$ \\
pH:Glucose & 2 & 0.1814 & 0.0907 & 10.125 & 0.000325 & $* * *$ \\
Oxygen:pH:Glucose & 2 & 0.1047 & 0.05236 & 5.845 & 0.006335 & $* *$ \\
Residuals & 36 & 0.3225 & 0.00896 & & & \\
\hline
\end{tabular}

Significance codes: $\quad \mathrm{p} \leq 0.001^{\text {'***', }}, 0.01^{\text {'**', }}, 0.05^{\text {‘* }}$ '

The impact of glucose concentration $(22.2 \mathrm{mM}$ vs. $11.1 \mathrm{mM})$ on fitness was assessed and not found to be a significant factor for fitness on its own across all genotypes (Table 5.7). However, glucose level was significant for MG1655 $l a c+\mathrm{pC} 12$, where I saw that fitness, on average, was higher in the low-glucose condition when compared to the regular glucose condition (Figure 5.3). At the beginning of these experiments, I predicted that limiting glucose availability might negatively impact fitness, as there could be further resource competition, and limiting glucose can have an impact on metabolism by providing a smaller pool of reducing power for production of ATP. Regardless of the overall impact of glucose on its own, it is evident that it becomes important more frequently when in combination with other factors (Table 5.7). 


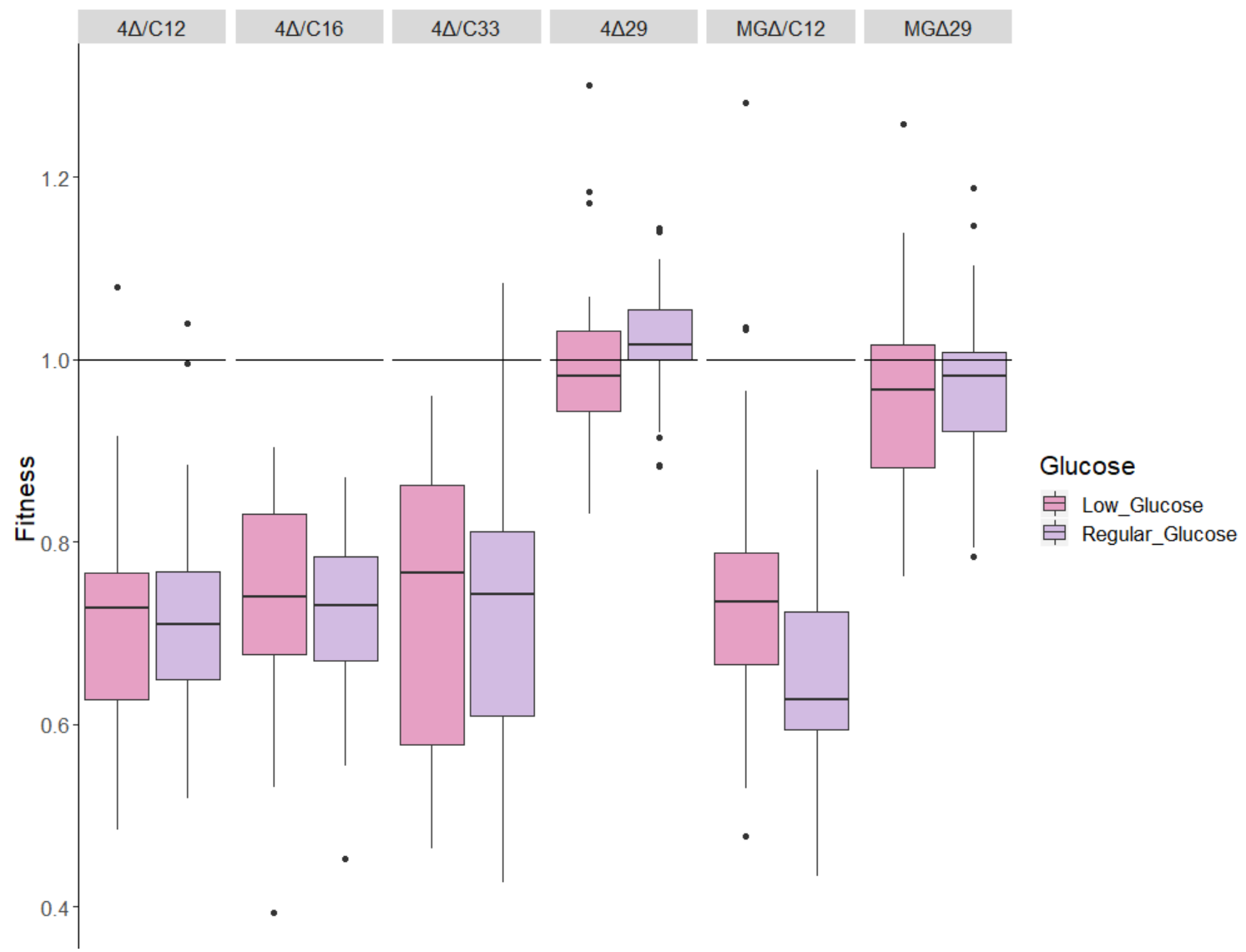

Figure 5.3: The average fitness of six host-plasmid combinations in media with low $(11.1 \mathrm{mM})$ or regular $(22.2 \mathrm{mM})$ glucose. Average relative fitness of MG1655 $\mathrm{lac}+\mathrm{pC} 16$, MG1655 $l a c+\mathrm{pPB} 29, \mathrm{~PB} 4 \Delta l a c+\mathrm{pC} 12, \mathrm{~PB} 4 \Delta l a c+\mathrm{pC} 16, \mathrm{~PB} 4 \Delta l a c+\mathrm{pC} 33$, and PB4 $l a c+$ pPB29 was measured against their respective plasmid-free ancestor in a headto-head competition assay in modified M9+glucose medium with phosphate buffer. Glucose was not a significant factor overall (5-way ANOVA of Fitness $\sim$ Host*Plasmid*Glucose* $\mathrm{pH}^{*}$ Oxygen, $\mathrm{p}>0.05$ ), but was significant for MG1655 $l a c+$ pC12 (3-way ANOVA of Fitness $\sim$ Glucose*pH*Oxygen, $\mathrm{p}<0.05$ ).

Finally, the impact of oxygen on the fitness of the strains was assessed (Figure 5.4). Like the other factors, fitness was not significantly impacted by the presence or absence of oxygen on its own when assessed for all genotypes (Table 5.7). Again, in that analysis, only plasmid identity and host, both of which are genetic factors, were the only 
individual factors found to be significant on their own. In combination with the other factors, the presence/absence of oxygen is a significant contributor to fitness in many, but not all, combinations of the other factors (Table 5.7). As I showed above, I broke down the significance of each factor for each genotype, and only found the presence/absence of oxygen to be significant for PB4 $l a c+p C 12$ (Table 5.6). In this host-plasmid combination, it is apparent that the average fitness for PB4 $l a c+p C 12$ is higher in the oxic vs anoxic condition, but such differences are less obvious in the remaining genotypes (Figure 5.3). While there were instances where fitness was lower in the anoxic environments, such as all strains but $\mathrm{PB} 4 \Delta l a c+\mathrm{pC} 33$ in the $\mathrm{pH} 8$, low-glucose medium (Table 5.7), this was by no means the rule. For example, MG1655 $l a c+p C 12$ was approximately equally as fit in media at $\mathrm{pH} 7$ with low or regular glucose in both the oxic and anoxic conditions. I also observed cases where fitness was seemingly better in the anoxic vs oxic conditions; for instance, all genotypes but MG1655 $\Delta l a c+\mathrm{pC} 12$ were more fit in the anoxic condition, when fitness was measured in the medium at $\mathrm{pH} 7$ with regular glucose. 


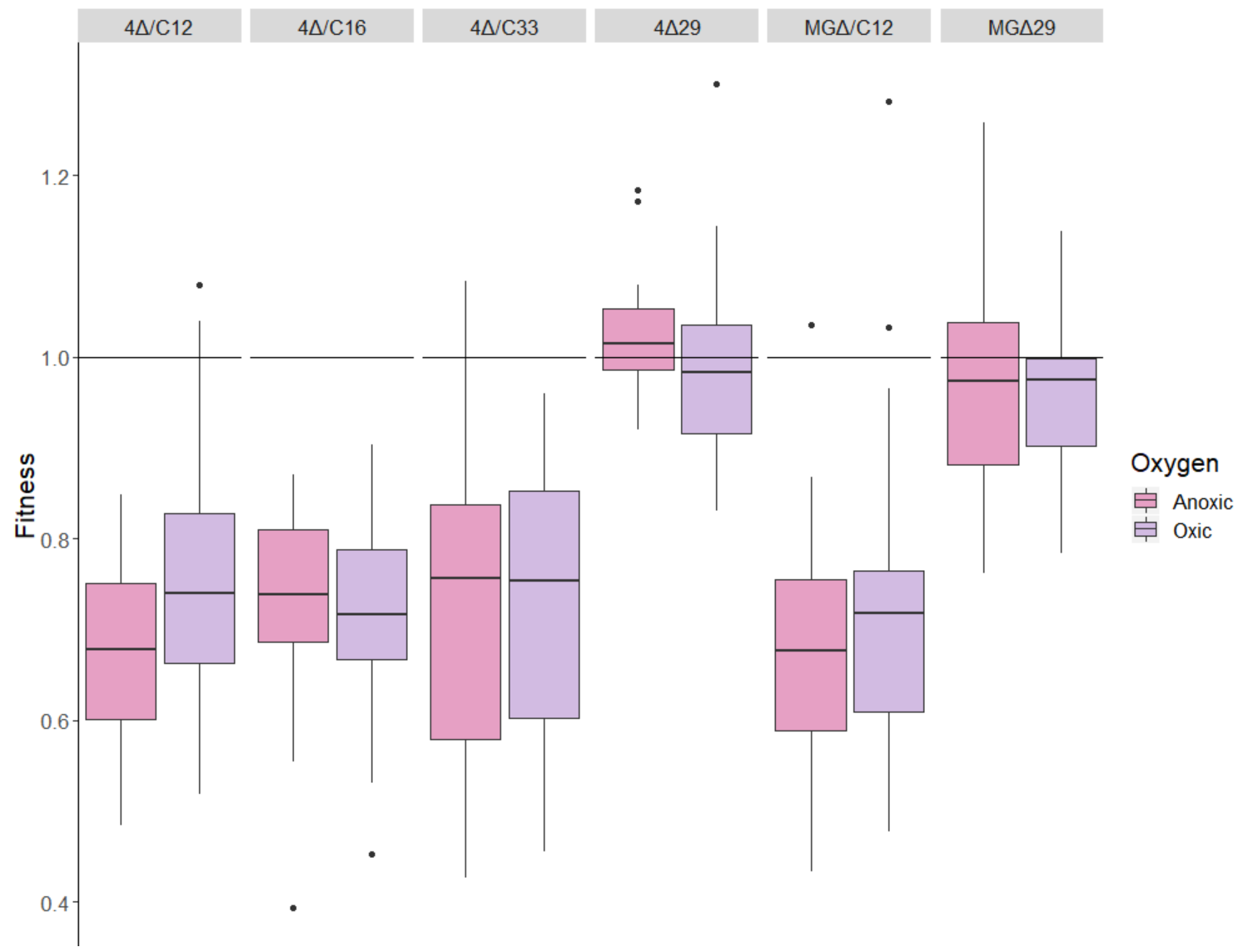

Figure 5.4: The average fitness of six host-plasmid combinations in the presence or absence of oxygen in modified M9+glucose media. Average relative fitness of MG1655 $l a c+\mathrm{pC} 16, \mathrm{MG} 1655 \Delta l a c+\mathrm{pPB} 29, \mathrm{~PB} 4 \Delta l a c+\mathrm{pC} 12, \mathrm{~PB} 4 \Delta l a c+\mathrm{pC} 16$, $\mathrm{PB} 4 \Delta l a c+\mathrm{pC} 33$, and PB4 $l a c+\mathrm{pPB} 29$ was measured against their respective plasmidfree ancestor in a head-to-head competition assay in modified M9+glucose medium with phosphate buffer. The presence or absence of oxygen was not a significant factor overall (5-way ANOVA of Fitness $\sim$ Host*Plasmid*Glucose*pH*Oxygen, $\mathrm{p}>0.05$ ), but was significant for PB4 $l a c+\mathrm{pC} 12$ (3-way ANOVA of Fitness $\sim$ Glucose* $\mathrm{pH}^{*}$ Oxygen, $\mathrm{p}<0.05)$. 
Table 5.6: 3-way ANOVA for the effect of three environmental factors on fitness for $\mathrm{PB} 4 \Delta l a c+\mathrm{pC} 12$.

\begin{tabular}{ccccccc}
\hline & Df & $\begin{array}{c}\text { Sum } \\
\text { Sq }\end{array}$ & $\begin{array}{c}\text { Mean } \\
\text { Sq }\end{array}$ & Fvalue & $\operatorname{Pr}(>\mathrm{F})$ & Significance \\
\hline \hline Oxygen & 1 & 0.0877 & 0.0877 & 6.55 & 0.0148 & $*$ \\
pH & 2 & 0.0687 & 0.03435 & 2.566 & 0.0909 & \\
Glucose & 1 & 0.0009 & 0.00091 & 0.068 & 0.796 & \\
Oxygen:pH & 2 & 0.0408 & 0.02038 & 1.522 & 0.2319 & \\
Oxygen:Glucose & 1 & 0.0224 & 0.02237 & 1.671 & 0.2043 & \\
pH:Glucose & 2 & 0.0056 & 0.00279 & 0.209 & 0.8126 & \\
Oxygen:pH:Glucose & 2 & 0.0665 & 0.03323 & 2.482 & 0.0977 & \\
Residuals & 36 & 0.482 & 0.01339 & & & \\
\hline
\end{tabular}

Significance codes: $\quad \mathrm{p} \leq 0.001^{\text {'***', }}, 0.01^{\text {(**', }}, 0.05^{\text {(*) }}$

\subsubsection{General Gene-by-Environment Interactions}

I examined GxE interactions for plasmid-bearing genotypes of E. coli in environments varying in glucose concentration, $\mathrm{pH}$, and oxygen availability. Overall, I found significant gene-by-environment interactions (Figure 5.5; Table 5.7). That is, there was a significant interaction of all gene (Host and Plasmid) and environment $(\mathrm{pH}$, Glucose, and Oxygen) terms and fitness using a five-way ANOVA with fitness as the dependent variable and host, plasmid, $\mathrm{pH}$, glucose, and oxygen as the independent variables $[\mathrm{F}(2,216)=6.915, p=0.001228]$. None of the environmental factors had an immediate impact on fitness by themselves, as indicated above; they instead contribute when in combination with some or all of the other factors. However, when in combination, there are several instances where they make a significant contribution to the resultant fitness outcome.

While the results of this 5-way ANOVA can illuminate the general trend of a GxE interaction, it is useful to further breakdown the interaction of only the environmental 
factors on fitness for each host-plasmid combination. MG1655 $\mathrm{Alac}+\mathrm{pC} 12$, $\mathrm{PB} 4 \Delta l a c+\mathrm{pPB} 29$, and $\mathrm{PB} 4 \Delta l a c+\mathrm{pC} 33$ each showed a significant interaction for Oxygen* $\mathrm{pH}^{*}$ Glucose on fitness (3-way ANOVA, Oxygen* $\mathrm{pH}^{*}$ Glucose, $\mathrm{p}<0.05$; Table 5.3, 5.4, and 5.6). MG1655 $l a c+\mathrm{pC} 12$ (Table 5.3) and PB4 $l a c+\mathrm{pC} 33$ (Table 5.5) both showed significant interaction terms for multiple factors or combinations of factors, however, for PB4+pPB29 (Table 5.4), the interaction term between all three factors is the only one to reach statistical significance. $\mathrm{PB} 4 \Delta l a c+\mathrm{pC} 12$ (Table 5.6) and $\mathrm{PB} 4+\mathrm{pC} 16$ (Table 5.8) did not have many instances where any individual factors or combinations of those factors were significant, except for the presence/absence of oxygen in $\mathrm{PB} 4 \Delta l a c+\mathrm{pC} 12$ and the interaction between Oxygen and Glucose for PB4 $\Delta l a c+\mathrm{pC} 16$. MG1655 $l a c+$ pPB29 did not show a significant interaction between the three factors, but instead showed significant interactions for $\mathrm{pH}$ alone, and combinations of Oxygen or $\mathrm{pH}$ with Glucose (Table 5.9). None of these factors, however, were the same across the six genotypes, which highlights that $\mathrm{GxE}$ interactions mediate fitness, rather than a single factor itself. 


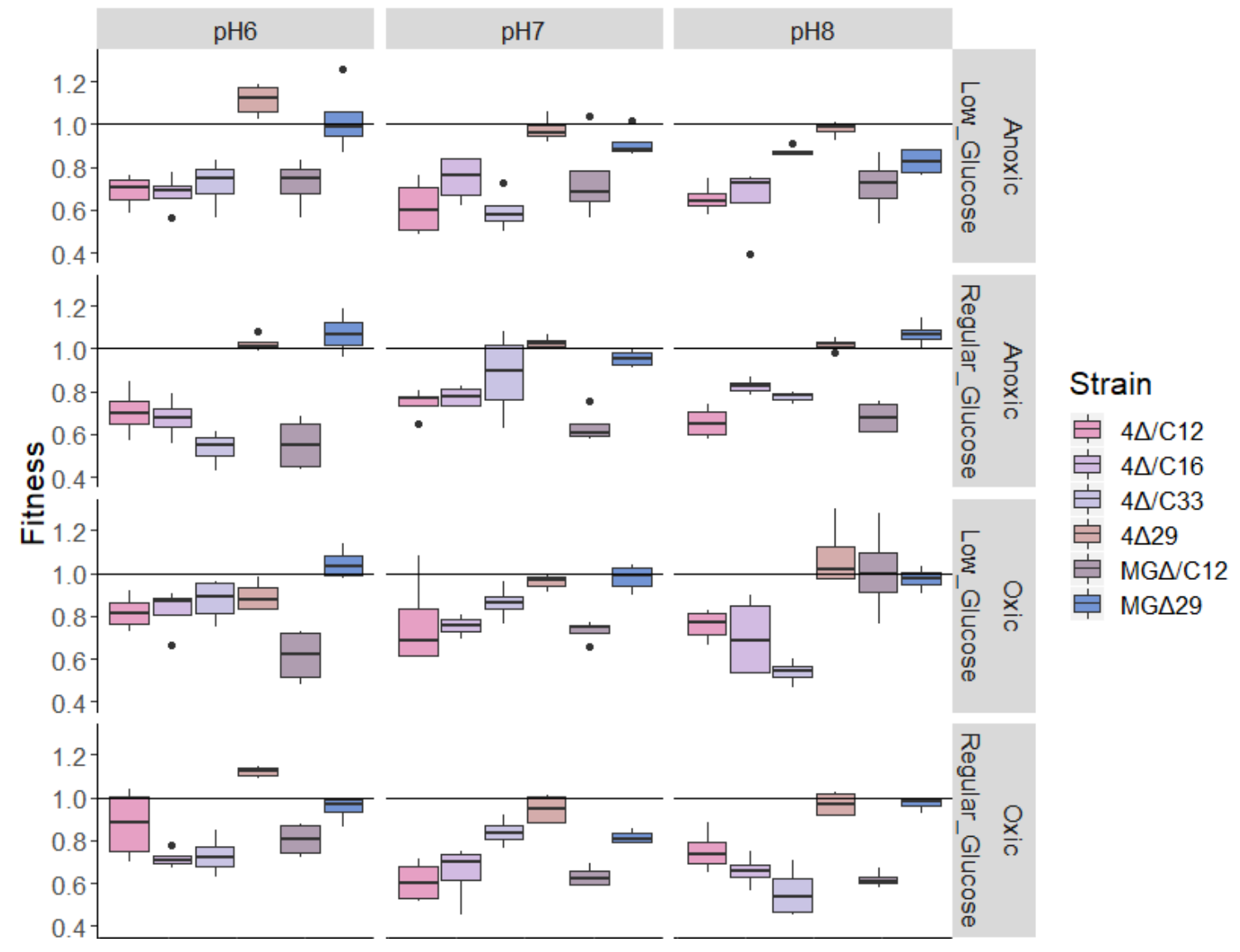

Figure 5.5: The relative fitness of six host-plasmid combinations in twelve environments. Average relative fitness of MG1655 $l a c+\mathrm{pC} 16, \mathrm{MG} 1655 \Delta l a c+\mathrm{pPB} 29, \mathrm{~PB} 4 \Delta l a c+\mathrm{pC} 12$, $\mathrm{PB} 4 \Delta l a c+\mathrm{pC} 16, \mathrm{~PB} 4 \Delta l a c+\mathrm{pC} 33$, and PB4 $l a c+\mathrm{pPB} 29$ was measured against their respective plasmid-free ancestor in a head-to-head competition assay in modified M9+glucose medium with phosphate buffer. The presence/absence of oxygen, $\mathrm{pH}$ (6.0, 7.0, and 8.0), and level of glucose (low $(11.1 \mathrm{mM})$ and regular $(22.2 \mathrm{mM}))$ were tested in the twelve environments. There was a significant interaction term for all host and environment terms (5-way ANOVA of Fitness $\sim$ Host*Plasmid*Glucose*pH*Oxygen, $\mathrm{p}<0.05$ ), where host and plasmid were the only significant factors on their own. 
Table 5.7: Five-way ANOVA testing the impact of five factors (host, plasmid, oxygen presence/absence, glucose, and $\mathrm{pH}$ ) on fitness. Factors and interactions which are significant are denoted in the right-most columns and significance codes are indicated below the table.

\begin{tabular}{|c|c|c|c|c|c|c|}
\hline & Df & Sum Sq & Mean Sq & F value & $\operatorname{Pr}(>\mathrm{F})$ & Significance \\
\hline Host & 1 & 0.101 & 0.1006 & 10.037 & 0.001756 & ** \\
\hline Plasmid & 3 & 4.545 & 1.5149 & 151.138 & $<2 \mathrm{e}-16$ & $* * *$ \\
\hline Oxygen & 1 & 0.017 & 0.0168 & 1.672 & 0.197384 & \\
\hline $\mathrm{pH}$ & 2 & 0.035 & 0.0176 & 1.758 & 0.17482 & \\
\hline Glucose & 1 & 0.018 & 0.0179 & 1.786 & 0.182772 & \\
\hline Host:Plasmid & 1 & 0.01 & 0.0097 & 0.973 & 0.325145 & \\
\hline Host:Oxygen & 1 & 0.001 & 0.0008 & 0.077 & 0.78232 & \\
\hline Plasmid:Oxygen & 3 & 0.126 & 0.042 & 4.191 & 0.006581 & $* *$ \\
\hline Host:pH & 2 & 0.063 & 0.0313 & 3.12 & 0.046173 & $*$ \\
\hline Plasmid:pH & 6 & 0.18 & 0.03 & 2.994 & 0.007868 & $* *$ \\
\hline Oxygen:pH & 2 & 0.078 & 0.0389 & 3.882 & 0.022061 & * \\
\hline Host:Glucose & 1 & 0.03 & 0.0299 & 2.984 & 0.085499 & \\
\hline Plasmid:Glucose & 3 & 0.059 & 0.0196 & 1.958 & 0.121328 & \\
\hline Oxygen:Glucose & 1 & 0.1 & 0.1004 & 10.019 & 0.001773 & $* *$ \\
\hline pH:Glucose & 2 & 0.002 & 0.0008 & 0.078 & 0.925075 & \\
\hline Host:Plasmid:Oxygen & 1 & 0.003 & 0.0033 & 0.332 & 0.565185 & \\
\hline Host:Plasmid:pH & 2 & 0.078 & 0.0391 & 3.901 & 0.021668 & * \\
\hline Host:Oxygen:pH & 2 & 0.117 & 0.0586 & 5.843 & 0.003378 & $* *$ \\
\hline Plasmid:Oxygen:pH & 6 & 0.435 & 0.0725 & 7.233 & 4.75E-07 & $* * *$ \\
\hline Host:Plasmid:Glucose & 1 & 0.033 & 0.0326 & 3.249 & 0.072878 & \\
\hline Host:Oxygen:Glucose & 1 & 0.005 & 0.0048 & 0.481 & 0.488549 & \\
\hline Plasmid:Oxygen:Glucose & 3 & 0.037 & 0.0124 & 1.239 & 0.296298 & \\
\hline Host:pH:Glucose & 2 & 0.044 & 0.022 & 2.193 & 0.114073 & \\
\hline Plasmid:pH:Glucose & 6 & 0.257 & 0.0429 & 4.278 & 0.000426 & $* * *$ \\
\hline Oxygen:pH:Glucose & 2 & 0.235 & 0.1174 & 11.711 & $1.48 \mathrm{E}-05$ & $* * *$ \\
\hline Host:Plasmid:Oxygen:pH & 2 & 0.005 & 0.0027 & 0.267 & 0.765675 & \\
\hline Host:Plasmid:Oxygen:Glucose & 1 & 0.078 & 0.0777 & 7.753 & 0.005837 & ** \\
\hline Host:Plasmid:pH:Glucose & 2 & 0.109 & 0.0543 & 5.417 & 0.005066 & ** \\
\hline Host:Oxygen:pH:Glucose & 2 & 0.085 & 0.0426 & 4.254 & 0.015418 & $*$ \\
\hline Plasmid:Oxygen:pH:Glucose & 6 & 0.104 & 0.0174 & 1.732 & 0.114723 & \\
\hline Host:Plasmid:Oxygen:pH:Glucose & 2 & 0.139 & 0.0693 & 6.915 & 0.001228 & ** \\
\hline Residuals & 216 & 2.165 & 0.01 & & & \\
\hline
\end{tabular}

Significance codes: $\mathrm{p} \leq 0.001^{\text {' } * * *}, 0.01^{\text {(**' }}, 0.05^{\text {‘* }}$ ' 
Table 5.8: 3-way ANOVA for the effect of three environmental factors on fitness for $\mathrm{PB} 4 \Delta l a c+\mathrm{pC} 16$.

\begin{tabular}{ccccccc}
\hline & Df & $\begin{array}{c}\text { Sum } \\
\text { Sq }\end{array}$ & $\begin{array}{c}\text { Mean } \\
\text { Sq }\end{array}$ & F value & $\operatorname{Pr}(>\mathrm{F})$ & Significance \\
\hline \hline Oxygen & 1 & 0.001 & 0.00098 & 0.084 & 0.7742 & \\
pH & 2 & 0.0044 & 0.00218 & 0.186 & 0.8312 & \\
Glucose & 1 & 0.0009 & 0.00092 & 0.078 & 0.7812 & \\
Oxygen:pH & 2 & 0.0608 & 0.03042 & 2.595 & 0.0885 & $*$ \\
Oxygen:Glucose & 1 & 0.0692 & 0.06922 & 5.905 & 0.0202 & $*$ \\
pH:Glucose & 2 & 0.0336 & 0.01681 & 1.434 & 0.2516 & \\
Oxygen:pH:Glucose & 2 & 0.0068 & 0.0034 & 0.29 & 0.7498 & \\
Residuals & 36 & 0.422 & 0.01172 & & & \\
\hline
\end{tabular}

$--$

Significance codes: $\quad \mathrm{p} \leq 0.001^{\text {'***, }}, 0.01^{\text {(***)}}, 0.05^{\text {(*) }}$

Table 5.9: 3-way ANOVA for the effect of three environmental factors on fitness for MG1655 lac+pPB29.

\begin{tabular}{ccccccc}
\hline & Df & Sum Sq & $\begin{array}{c}\text { Mean } \\
\text { Sq }\end{array}$ & F value & $\operatorname{Pr}(>\mathrm{F})$ & Significance \\
\hline \hline Oxygen & 1 & 0.00499 & 0.00499 & 0.859 & 0.3602 & \\
pH & 2 & 0.09298 & 0.04649 & 8.003 & 0.00133 & $* *$ \\
Glucose & 1 & 0.00189 & 0.00189 & 0.326 & 0.57164 & \\
Oxygen:pH & 2 & 0.01359 & 0.00679 & 1.169 & 0.32205 & $* * *$ \\
Oxygen:Glucose & 1 & 0.11732 & 0.11732 & 20.196 & $6.97 \mathrm{E}-05$ & $* *$ \\
pH:Glucose & 2 & 0.07411 & 0.03706 & 6.379 & 0.00425 & $* *$ \\
Oxygen:pH:Glucose & 2 & 0.00549 & 0.00274 & 0.472 & 0.62729 & \\
Residuals & 36 & 0.20913 & 0.00581 & & & \\
\hline
\end{tabular}

Significance codes: $\quad \mathrm{p} \leq 0.001^{\text {'**** }}, 0.01^{\text {(***, }}, 0.05^{(*}$ '

\subsubsection{Effect of nitrate-supplementation on fitness in anoxic conditions}

In some cases, costs of plasmid carriage were higher under anoxic conditions (Figures 5.4 and 5.5). To assess if providing an alterative terminal electron acceptor for the anoxic competitions would mediate any energy output related costs due to the absence of cellular respiration, nitrate was added to the competitions where fitness 
appeared to be lower in the anoxic condition $(\mathrm{PB} 4 \Delta l a c+\mathrm{pC} 33$ in $6 \mathrm{~L}$, MG1655 $l a c+\mathrm{pC} 12$ in $8 \mathrm{~L}$ ), and fitness was measured again in both environments (Figure 5.6). Within this figure, I show the fitness that I initially measured in my GxE experiments above for the given environment with or without oxygen ("Original"), and the fitness of the same strain in the same environments measured in the experiments with and without nitrate supplementation. Values for the "Original" and "0mM Nitrate" should be similar, as they are measured in the same conditions without nitrate. There were no significant differences detected following the addition of the nitrate overall, such that fitness did not improve following the addition of 10mM nitrate (One-way ANOVA; Fitness Condition, Tukey's posthoc, $p$-adj $>0.05)$. Note that in the case of both the "original" and 0mM nitrate conditions, fitness was higher in the oxic condition than in the anoxic condition. The trend for PB4 $l a c+\mathrm{pC} 33$ is consistent, in that I see fitness across all anoxic and oxic conditions were not significantly different regardless of whether nitrate was added or not. However, for MG1655 $l a c+\mathrm{pC} 12$, it is evident that fitness was higher in the $0 \mathrm{mM}$ nitrate competition than in the original competitions done in $8 \mathrm{~L}$ with and without oxygen. Instead, fitness for the strain tested in both conditions with the addition of $10 \mathrm{mM}$ nitrate was closer to the initial fitness measurement. This suggests two things: 1) the repeatability is questionable for this case, though it is interesting that the addition of nitrate brings fitness to a similar level, and 2) if the original measurement is ignored for a moment, the addition of nitrate apparently decreased fitness, though not significantly, when compared to the fitness measurement without nitrate. These findings suggest that the increased costliness of both plasmids in the anoxic environment is not solely a response to the lower energetic output by E. coli 
during fermentation. If this were the case, I expected that the addition of nitrate would result in the fitness between the oxic and anoxic conditions to be the same.

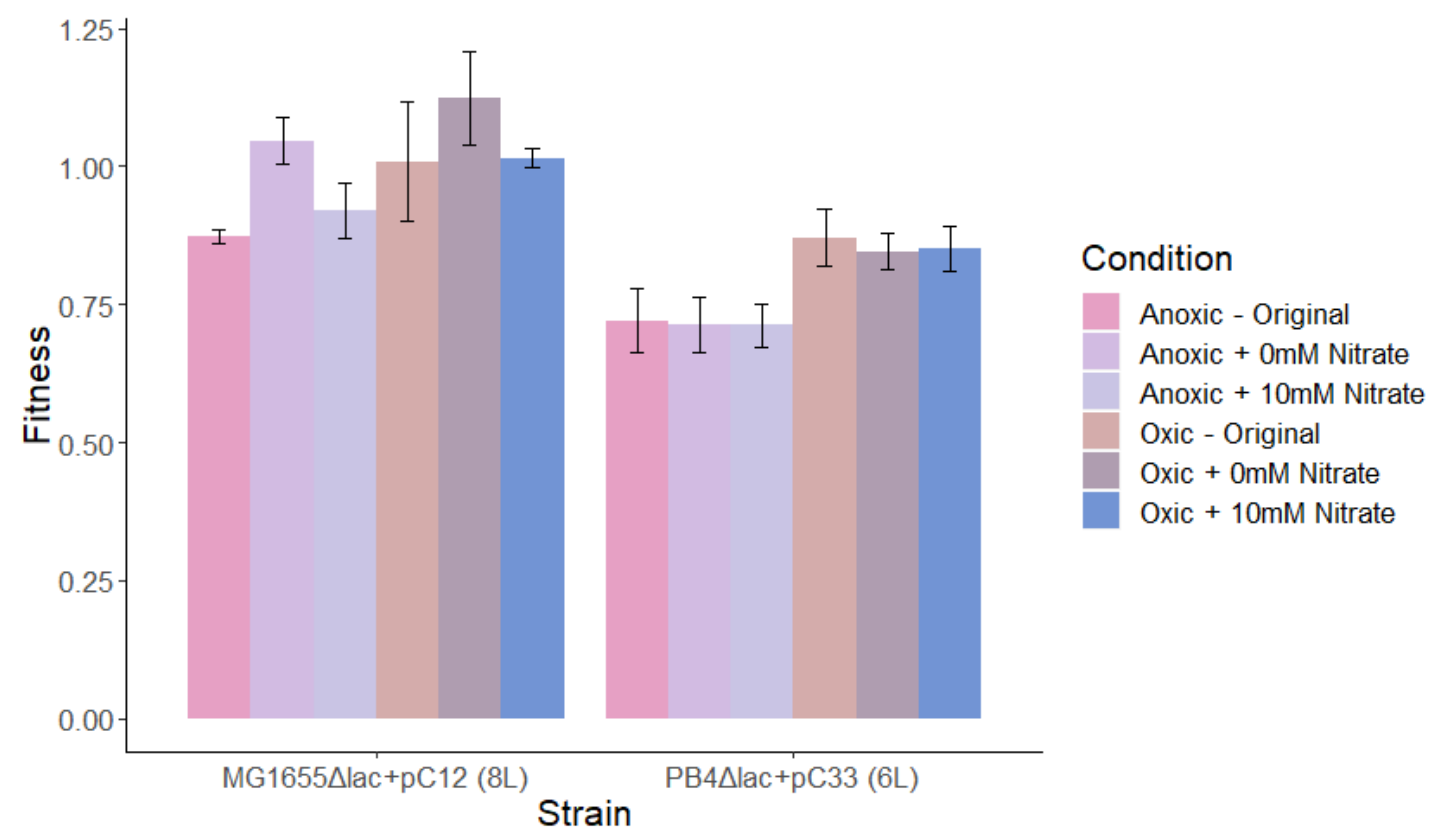

Figure 5.6: Relative fitness of MG1655 $l a c+\mathrm{pC} 12$ and $\mathrm{PB} 4 \Delta l a c+\mathrm{pC} 33$ grown in modified M9 medium with and without nitrate supplementation $(10 \mathrm{mM})$ both with and without oxygen. MG1655 $\mathrm{lac}+\mathrm{pC} 12$ was grown in modified M9 medium at $\mathrm{pH} 8.0$ with low $(11.1 \mathrm{mM})$ glucose $(8 \mathrm{~L})$ and $\mathrm{PB} 4 \Delta \mathrm{lac}+\mathrm{pC} 33$ was grown in modified $\mathrm{M} 9$ medium at pH 6.0 with low $(11.1 \mathrm{mM})$ glucose $(6 \mathrm{~L})$. Measures represent the initial fitness measure ("Original") in previous experiments, and the oxic/anoxic conditions with and without nitrate supplementation are fitness measures found in separate experiments. Fitness across conditions (presence or absence of oxygen) was not found to be significantly different with or without nitrate supplementation (One-way ANOVA; Fitness $\sim$ Condition, Tukey's posthoc, $p$-adj>0.05).

\subsubsection{Impact of environment on drug resistance}

Since environment may also have an impact on drug resistance, I measured changes in resistance to kanamycin for strains carrying pPB29, and changes in resistance 
to meropenem for hosts carrying the carbapenemase plasmids in all conditions. There were notable patterns in resistance observed for these plasmid-carrying strains (Figure 5.7). For example, in MG1655 $\Delta$ lac + pPB29 (Fig.5.7A), as $\mathrm{pH}$ increased from 6 to 8 , the MIC towards kanamycin decreased. MICs for this strain and drug were lower in oxic condition than in the anoxic condition. This pattern persisted regardless of glucose level. This pattern only appears to hold true for this pPB29-carrying strain, as neither $\mathrm{pH}$ nor oxygen had consistent effects on kanamycin MICs for PB4Alac+pPB29 (Fig. 5.7B).

For the carbapenemase-carrying strains MG1655 $\Delta$ lac + pC12 (Fig.5.7C),

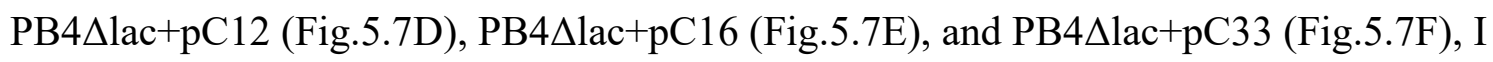
find the opposite pattern. Here, MICs to meropenem increase as $\mathrm{pH}$ increases, such that strains grown at higher $\mathrm{pH}$ are more resistant to the drug and in the case of

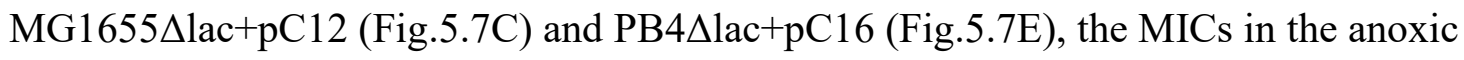
condition are lower than those for the oxic condition. For PB4 $\Delta \mathrm{lac}+\mathrm{pC} 12$ (Fig.5.7D) and PB4 4 lac+pC33 (Fig.5.7F), MIC increased as pH did, but MIC was nearly identical between the oxic and anoxic conditions. This variation in MIC further indicates the importance of gene-by-environment interactions, such that these interactions not only influence fitness, but can also mediate the change in resistance. 

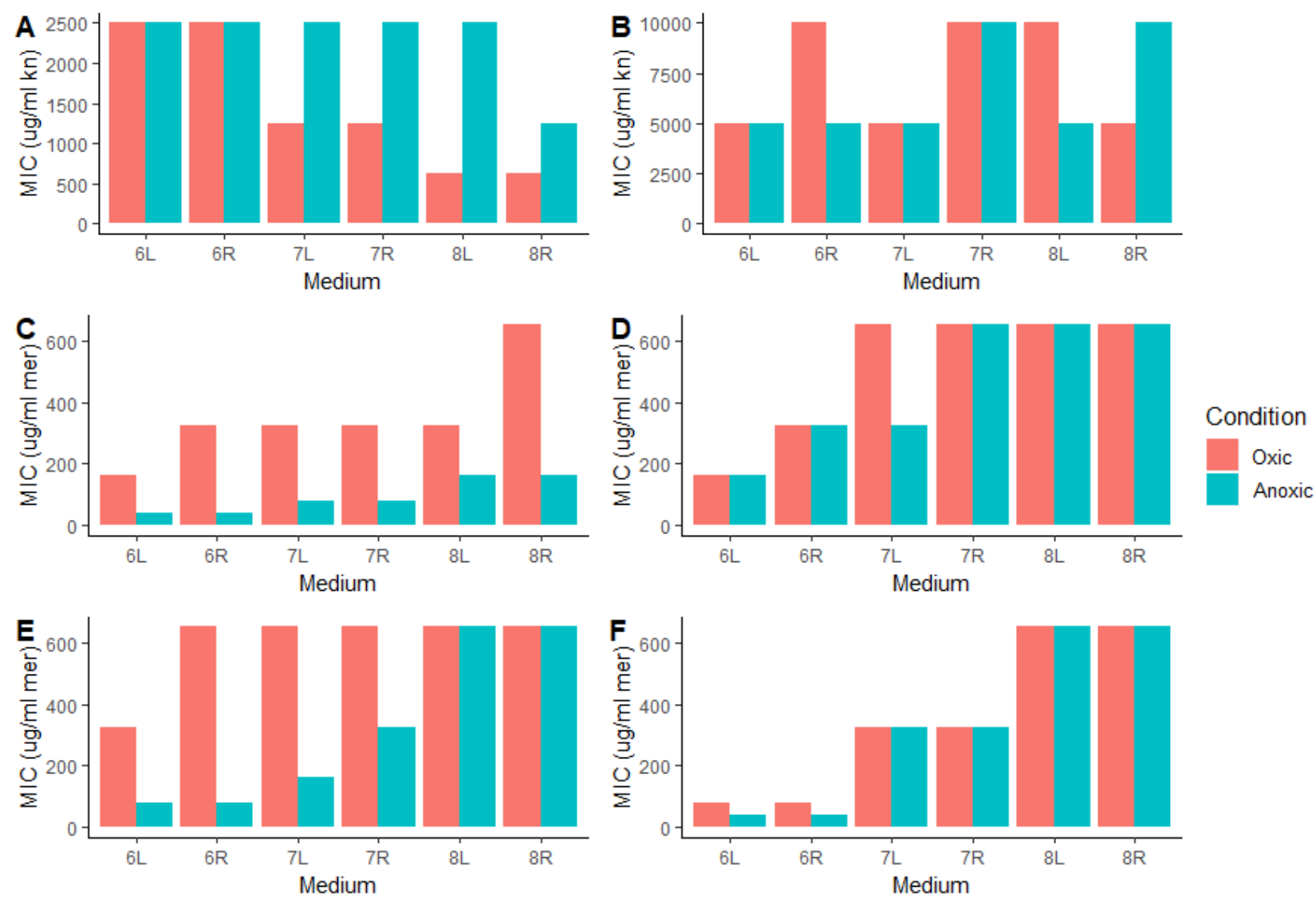

Figure 5.7: Change in minimum inhibitory concentration (MIC) for six host-plasmid combinations measured in twelve environments. MIC ( $\mu \mathrm{g} / \mathrm{ml}$ kanamycin or meropenem)

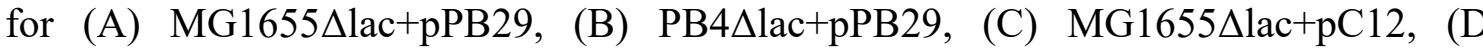

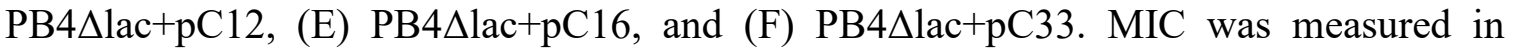
duplicate across each environment, a modified M9+glucose medium $(\mathrm{pH} \mathrm{6,} \mathrm{7,} \mathrm{or} 8$ with low (L) or regular (R) glucose) in the presence and absence of oxygen.

Resistance to both kanamycin and meropenem appeared to vary as $\mathrm{pH}$ changed, with resistance either increasing or decreasing with $\mathrm{pH}$ for meropenem and kanamycin, respectively. Changes in resistance to both appeared to be related to whether the MIC was measured in the presence or absence of oxygen. As such, I sought to investigate if the electron transport chain (ETC) might be responsible for this pattern, especially for kanamycin. Because MG1655 $\mathrm{lac}+\mathrm{pPB} 29$ was more susceptible to the drug in the oxic 
condition, this might suggest that the ETC, which is not used during fermentation, might be responsible. Similarly, if resistance changed with $\mathrm{pH}$, this could also point to the ETC and other factors which would shuttle drug into the cell. A further review of the literature suggested that this variation in resistance to kanamycin is likely to be a result of the ETC, whereas resistance to meropenem was likely due to other factors (discussed below).

Since I observed a striking trend for MG1655 $\Delta \mathrm{lac}+\mathrm{pPB} 29$, where resistance decreased as $\mathrm{pH}$ increased, especially in the oxic environments and predicted that the ETC was responsible, pPB29 was transferred to four ETC mutants and their susceptibility to kanamycin was measured (Figure 5.8). Note that I initially did attempt to transfer the carbapenemase plasmids into the ETC mutants and was ultimately unsuccessful; however, the literature points to ETC being responsible for the patterns of kanamycin resistance observed with pPB29 and not necessarily for the carbapenemase plasmids, so this kind of investigation might not have been useful (Schlessinger 1988; DeMars et al. 2016). As discussed above, I saw a pattern for MG1655 lac+pPB29 where resistance decreased as $\mathrm{pH}$ increased, and anoxic MIC values were frequently higher than the MIC for the oxic condition (Figure 5.8). I see this pattern repeat broadly for the four ETC mutants (Figure 5.8), where resistance decreases with $\mathrm{pH}$, and varied between the oxic and anoxic conditions. However, these patterns are less consistent compared with MG1655 $\Delta$ lac+pPB29. The Gn12 mutant (Figure 5.8F) looks to be most like the pattern first observed for MG1655 $\Delta \mathrm{lac}+\mathrm{pPB} 29$, but the patterns for the remaining three are much less obvious. In these cases, the difference between oxic and anoxic conditions at $\mathrm{pH} 7$ and 8 are not as obvious, nor are the changes with $\mathrm{pH}$ as clear. It appears as if 1) the $\mathrm{pH}$ dependence, while still evident, does not seem as strong and 2) the differences between 
oxic and anoxic measurements become less variable. Since there was less variation in resistance for the ETC mutants as $\mathrm{pH}$ changed with the presence or absence of oxygen, this suggests that ETC, at least to some degree, is responsible for the initial differences in kanamycin resistance observed for MG1655 $\mathrm{lac}+\mathrm{pPB} 29$. 

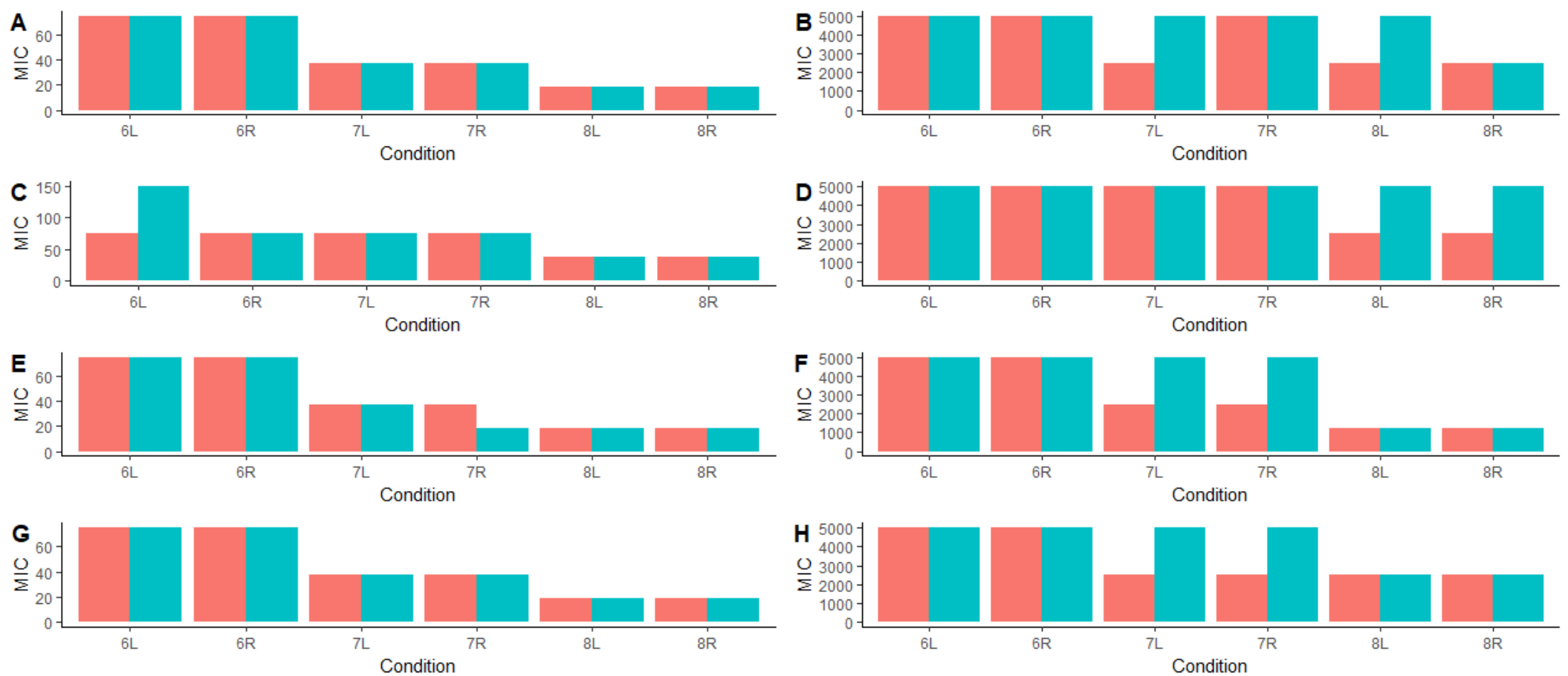

Figure 5.8: Change in minimum inhibitory concentration (MIC) for four strains of E. coli bearing electron transport chain (ETC) mutations with plasmid pPB29, as measured in twelve environments. MIC (ug/ml kanamycin) for four ETC mutants with pPB29. (A) Kn14, (B) Kn14+pPB29, (C) Kn15, (D) Kn15+pPB29, (E) Gn12, (F) Gn12+pPB29, (G) Gn14, and (H) Gn14+pPB29. MIC was measured in duplicate across each environment, a modified M9+glucose medium ( $\mathrm{pH}$ 6, 7, or 8 with low (L) or regular (R) glucose) oxically or anoxically. 


\subsection{Discussion}

In this study, I aimed to assess gene-by-environment interactions between AMR E. coli and their growth environments, focusing on the effects of $\mathrm{pH}$, glucose concentration, and the presence/absence of oxygen. Overall, I found GxE interactions amongst the combined environmental factors ( $\mathrm{pH}$, glucose, and oxygen) and the six strains tested here (Table 5.7; Figure 5.5). I expected that there would be a general response where the relative fitness of each strain would vary with environment, as identified in previous reports (Hall 2013; Clarke et al. 2020; but see Gifford 2016).

Further, these results show that carbapenemase-encoding plasmids are generally costly to their E. coli hosts, regardless of environment (Figure 5.1). This contrasts with another study that measured the effect of the two carbapenemase plasmids, pG12-KPC-2 and pG06-VIM-1, which were not very costly (1.1-3.6\% fitness reduction) in their E. coli hosts following transfer from K. pneumoniae (Di Luca et al. 2017).

I expected that at least some of the abiotic factors might have an impact on fitness and that relative fitness would vary as these factors were varied, particularly when the factors are shifted away from what is considered optimal. More specifically, I did not expect that the relative fitness would remain the same between each environment (e.g., Strain A>Strain B in all cases with absolute fitness changing with environment), but would instead change between environments, such that Strain A might be more fit than Strain B in one environment, but the opposite can be seen in another. Across all genotypes, none of the environmental factors was significant on its own, but rather the genetic factors (Host and Plasmid) were found to be significant (Table 5.7). There was a significant interaction term between all genetic and environmental factors on fitness, and 
some lower-order combinations of these factors were also found to be significant (Table 5.7). These findings show the importance of gene-by-environment interactions overall, and that some genotypes will interact differently with individual environmental factors to produce a particular fitness outcome.

E. coli is a facultative anaerobe, able to survive in the presence or absence of oxygen, although ATP production is less under anoxic conditions using fermentation, when compared to aerobic or anaerobic respiration (Unden and Trageser 1991; Gunsalus and Park 1994; Finn et al. 2017). Plasmid costliness has been proposed to be a result of the energetic demands on the host from plasmid maintenance, which may reduce its ability to accurately complete its own necessary functions because resources are depleted or sequestered away for plasmid use (San Millan and MacLean 2017). Since I observed cases where fitness was lower in the anoxic environment, the initial energetic cost of the plasmid may have been further exacerbated by reducing the energy output of the cell. To reiterate, oxygen presence/absence was not found to be a significant contributor on its own across all genotypes. Oxygen presence/absence was only a significant predictor of fitness for $\mathrm{PB} 4 \Delta l a c+\mathrm{pC} 12$. However, it was found to be significant in conjunction with other factors, both genetic and environmental. Some strains of E. coli, like MG1655 and PB4, have nitrate reductase, which allows for the use of nitrate as the terminal electron acceptor for anaerobic respiration. I expected that if fitness deficits were increased due to the lowered energy outputs in the absence of respiration, the addition of nitrate would alleviate this effect (Stewart et al. 2002). However, supplementation of the media with nitrate did not significantly improve fitness in the anoxic condition for either of the host- 
plasmid combinations tested, so it is unlikely that energetic burdens are the sole reason for such costs (Figure 5.6).

Glucose was used as both a carbon source and an energy source in the media formulations (Shehata and Marr 1971; Gunsalus and Park 1994; Finn et al. 2017). It also acts as an electron donor, where the electron pool can be used in further reactions during respiration. In oxic conditions, glucose is a limiting factor for respiration, but in microaerobic conditions, oxygen becomes limiting, even when the glucose concentration is low (Henkel et al. 2014). Lower concentrations of glucose would eventually become detrimental to the cell under both oxic and anoxic conditions, as there is a smaller pool of carbon, electrons, and ultimately ATP, available as the bacteria continue to grow and utilize the available carbon supply. For instance, E. coli is known to grow more slowly in lower concentrations of glucose, and genes related to alternate glucose transport pathways and phosphorylation are upregulated (Olvera et al. 2009). Facultative anaerobes, like E. coli, are also known to utilize different transport systems to import glucose in the absence of oxygen, and one such system, the glucose phosphotransferase system (PTS), is less effective at transport in glucose-limited conditions (Manché et al. 1999). This would suggest that the cell will generally be impacted in glucose-limited conditions but might be further impacted when energy outputs are already decreased using fermentation versus aerobic or anaerobic respiration. Limiting glucose levels can consequently affect cAMP levels, which is responsible for regulating the response of $E$. coli to various sources of nutrients (e.g., growth in excess glucose typically correlates with decreased cAMP expression versus growth at limiting glucose concentrations) (Notley-McRobb et al. 1997). 
On the other hand, glucose also plays a role in the anabolic processes in E. coli. That is, the production of various macromolecules by the cell can require the use of intermediates of glucose from the metabolic pathways (Madigan et al. 2018). Such intermediates are frequently taken from glycolysis or the citric acid cycle, and can be used for producing amino acids, the building blocks of peptidoglycan, as well as other polysaccharides (Madigan et al. 2018). As such, lower glucose availability could also negatively affect the cell, as there would be few glucose molecules available to produce these essential intermediaries, possibly resulting in a decreased growth rate by the cell (Russell and Cook 1995). Further, environment, specifically the presence or absence of oxygen and $\mathrm{pH}$, can also impact the types of metabolic products which might be required (Tweeddale et al. 1998). What this means is that the availability of glucose, sometimes in combination with other environment factors (like those tested here), can not only effect catabolism, but can also impact anabolism and cell growth.

I expected that limiting the available concentration of any nutrient would negatively impact growth and increase resource competition generally, such that absolute fitness will be decreased in the lower-glucose environment. This, however, does not necessarily mean that relative fitness would be affected by the decrease in glucose concentration. Nonetheless, there was not an overall impact of glucose level on fitness of these strains, but is instead significant in combination with other factors (Table 5.7) (Shehata and Marr 1971). The fitness of MG1655 $l a c+\mathrm{pC} 12$ was significantly impacted by the concentration of glucose on its own, however, which indicates that it is still an important factor to continue investigating. While I did not test an extremely low concentration in my "low" glucose concentration, lower concentrations of glucose would 
presumably be increasingly detrimental via the decreased carbon and energy supply, which can impair the energetic output of the cell, and limit cell growth. In such cases, I would have predicted that costly host-plasmid combinations, which may have already been at a growth disadvantage relative to its more fit competitor, would grow more slowly still, and increase the observed fitness costs.

$\mathrm{pH}$ was not found to be a significant factor on its own, but only when in combination with other factors when considering all genotypes (Table 5.7). By genotype, this factor was observed to be significant on its own in half of the host-plasmid combinations. $\mathrm{pH}$ is an interesting environmental factor for several reasons; first, there is some evidence showing that the absolute fitness of the tested bacteria was lowered by acidic conditions (Kishony and Leibler 2003). This shift from a neutral $\mathrm{pH}$ to either a more acidic or basic environment is expected to have some effect on the cell, not only through changes to the structure and function of macromolecules, such as essential enzymes and proteins, but also through interference with proton motive force (Jin and Kirk 2018). Further, $\mathrm{pH}$ is important in many potential environmental reservoirs. For example, $\mathrm{pH}$ varies in the gastrointestinal tract or in various stages of wastewater treatment, and therefore it is quite useful to understand the impact of $\mathrm{pH}$, alone and with other conditions, that can be relevant to environments plagued by antimicrobial resistance (Evans et al. 1988; Koziolek et al. 2015).

Since I found that environment is an important contributor to fitness, I was then interested in testing the effect of environment on resistance. The impact of environment on resistance was demonstrated here, with two patterns emerging for pPB29 and the carbapenemase-encoding plasmids (Figure 5.7). pPB29, which was assayed with the 
aminoglycoside drug kanamycin, showed a trend in resistance that has been previously reported in the literature, where resistance decreases with $\mathrm{pH}$ and are higher in anoxic conditions (Schlessinger 1988). Lower $\mathrm{pH}$ and anoxic growth is thought to decrease the proton motive force (PMF), which results in decreased influx of the drug, and thus it is unable to exert its antimicrobial effects (Schlessinger 1988; DeMars et al. 2016). For two carriers of carbapenemase-encoding plasmids, resistance tended to increase with $\mathrm{pH}$, a phenomenon also accounted for in the literature (Figure 5.7). One study showed a significant decrease in $\beta$-lactamase activity in $\mathrm{pH} 5.8$ versus $\mathrm{pH} 7.5$ (Ohsuka et al. 1995) and another found that $E$. coli was more susceptible to $\beta$-lactams, among other drug classes, in anoxic environments, likely because of slower growth rates in the anoxic condition (DeMars et al. 2016). This slower growth rate impacts the synthesis of the cell wall, making it more susceptible to the action of $\beta$-lactam drugs, which typically exert their activity by interfering with cell wall synthesis (DeMars et al. 2016).

$\mathrm{pH}$ will also have an impact generally on antibiotic activity itself, in addition to impacting the permeability of the membrane. External $\mathrm{pH}$ is known to influence the protonation and deprotonation states of antibiotics (Delcour 2009). One study found that aminoglycosides work best in alkaline conditions and many $\beta$-lactams work best in acidic conditions (Yang et al. 2014). Aminoglycoside activity is inhibited by the addition of cations, which inhibit ionic binding of the drug to the membrane, thus interfering with drug influx (Blaser and Lüthy 1988). Additionally, at physiological pH, aminoglycosides have a net-positive charge, which allows them to easily enter the cell due to the electrical potential difference between the inside and outside of the cell; when $\mathrm{pH}$ is decreased out 
of the cell, this potential is decreased and passage of the drug into the cell is impaired (Blaser and Lüthy 1988).

Finally, pH may also influence the activity of aminoglycoside acetyltransferases (such as the one produced by pPB29) through substrate inhibition of the enzyme, which in turn can diminish the effects of the enzyme and increase the activity of the drug and its ability to kill the cell (Culebras et al. 1996). This is important to note, because this helps to explain the patterns I observed; pPB29 encodes aac(3)-IIA and aac(6')-Ib-cr, aminoglycoside acetyltransferases which give resistance to aminoglycosides, are localized in the cytoplasm (Vliegenthart et al. 1991). This means $\mathrm{pH}$ not only impacts drug entry, either increasing or decreasing entry, but once the drug is able to enter, these enzymes should be able to exert their resistance effects; however, if the drug is able to enter the cell, but the enzyme is not acting efficiently due to substrate inhibition, the drug might be able to act more effectively against the cell.

$\beta$-lactams, on the other hand, may be more active at lower $\mathrm{pH}$ in part because penicillin binding protein (PBP) 2 a changes conformation in a way that makes the protein more susceptible to the drug (Delcour 2009). Protonation of $\beta$-lactams in acidic conditions can also help to activate these drugs and stimulate their activity at the active site (Atanasov et al. 2000; Lemaire et al. 2008; Delcour 2009). Not surprisingly, pH can also change membrane permeability generally, which further acts to control how drug might enter the cell. For example, porins will close in acidic conditions, which can impact selectivity and entrance of some drugs (Delcour 2009). 
Since the ETC is implicated in the patterns of susceptibility observed for pPB29, assessing susceptibility with ETC mutants could confirm the role of the ETC in these varied MICs across $\mathrm{pH}$ and the oxic vs anoxic condition. I tested changes in susceptibility with four ETC mutants, and I observed that resistance to kanamycin started to decrease at $\mathrm{pH} 8$, rather than beginning to decrease at $\mathrm{pH} 7$ and then again at $\mathrm{pH} 8$ (Figure 5.8). This indicates that the pattern of susceptibility observed for pPB29 was likely to due to the ETC and its effect on influx of drug in increasing $\mathrm{pH}$ environments. Since drug influx was impaired in strains with a faulty ETC, the strains were more resistant to kanamycin, even at higher $\mathrm{pH}$ points ( 7 and 8 ) where they were increasingly more susceptible prior to introduction of the plasmid. While these patterns have been documented previously, it shows that such changes in resistance also apply to plasmid-borne resistance. Given the prevalence of AMR plasmids, investigating all avenues which might increase their susceptibility is essential. As carbapenemase-encoding plasmids are becoming more prevalent, it would be useful to conduct further analyses like this one to assess how and why resistance varies in different environments. Ideally, these studies can lead to the discovery of situations where resistance might be decreased.

Recently, Clarke et al (2020) found evidence of GxE interactions in six AMR mutants of $E$. coli in ten environments, varying from lab-based media (LB and M9+Glucose), to environmental media (ex. river and soil), as well as media mimicking urine and colon conditions. This work highlights that fitness measurements taken in lab media, such as LB, does not necessarily capture the fitness of that strain in a different environment, especially ones that might be relevant to human health and the broader environment. By expanding on this work, I have found that the fitness of plasmid-bearing 
antimicrobial resistant E. coli is a result of GxE interactions. This highlights the need to test beyond the standard laboratory media, such as minimal media or LB broth, for estimating the fitness of AMR pathogens. Further, this work is particularly novel, in that I not only investigated the presence or absence of GxE interactions for plasmid-carrying bacteria, but I also attempted to identify which specific factors impacted the fitness of these AMR mutants. By defining specific factors (i.e., presence/absence of oxygen, $\mathrm{pH}$, and level of glucose), and varying them in a systematic way, it becomes easier to assess which factors or which levels of these factors are particularly relevant in determining fitness and resistance.

Understanding the broader implications of GxE interactions can be essential to understanding why plasmids may persist as they do, beyond the typical means of transfer, selection, and compensatory evolution, in part because certain environments may make their carriage more favourable than others. Similarly, it contributes to the idea that there likely exist various environmental reservoirs which can act as refuges for AMR pathogens, which then have the potential to infect humans and animals. Many of the environmental factors and levels, such as presence or absence of oxygen or $\mathrm{pH}$, were chosen because they very broadly mimic some aspects of the GI tract, and these results might inform further work which might be able to leverage any weakness of some hostplasmid combinations when in certain environments. For example, the $\mathrm{pH}$ in the human stomach can range between $\mathrm{pH}$ 1.7-4.7, although those were not measured here, the small intestine can range between $\mathrm{pH}$ 5.9-6.3 in the proximal portions to $\mathrm{pH}$ 7.4-7.8 in the distal ones, with colonic $\mathrm{pH}$ conditions able to fluctuate between $\mathrm{pH}$ 5-8 (Koziolek et al. 2015). As such, the range of $\mathrm{pH}$ tested here $(\mathrm{pH} 6, \mathrm{pH} \mathrm{7}$, and $\mathrm{pH} 8)$ encapsulates those 
that are observed after the stomach and can help us understand the fitness and resistance of pathogens in this environment. More importantly, these results could be used as a starting point to discover which environmental factors can be manipulated in wastewater treatment or food production plants, for example, to reduce the number of AMR bacteria that might exist in those environments. 


\section{Chapter 6: Conclusions and Future Directions}

Antimicrobial resistance (AMR) is a public health threat that has the potential to dramatically and negatively impact the quality of treatment for bacterial infections.

Plasmids, mobile genetic elements capable of transfer between strains, are a way that AMR can be spread within and between populations. As such, understanding the areas of plasmid biology which are still not well characterized (e.g., plasmid persistence and various kinds of interactions) will go a long way in not only understanding how and why plasmids persist the way they do, but will hopefully give us a place to start to combat plasmid-mediated AMR.

In my first data chapter, Chapter 3, I investigated gene-by-gene interactions between six E. coli hosts and plasmid pPB29 and investigated the long-term impact that this initial fitness value had on the mechanism used to maintain plasmids for 100 generations. In the literature, there is evidence that fitness can vary for a single plasmid in different hosts, though this is by no means the rule (discussed in Chapter $\mathbf{2}$ and the references therein). I observed epistatic interactions between the plasmid pPB29 and the six E. coli hosts into which I transferred the plasmid. Theory states that when plasmids are costly, they should be lost due to purifying selection, although compensatory evolution and conjugation can help rescue costly plasmids from this fate (Bergstrom et al. 2000; Harrison and Brockhurst 2012). Indeed, when in mixed culture, pPB29 was conjugated to the plasmid-free competitor when the initial donor experienced a cost. Instead of being lost completely, the plasmid was maintained in a different host in these cases, when the initial donor was lost. There was also some evidence that compensatory evolution can help maintain the plasmid in monoculture, but this was not common. This 
shows that plasmids, despite their initial costs, can readily be maintained in populations over time, rather than being lost to purifying selection.

In my second data chapter, Chapter 4, I evolved three strains of $E$. coli, each carrying pPB29, for 500 generations in three selection conditions (LB, Kn0.1, and $\mathrm{Kn} 10$ ). Plasmids have been found to persist for upwards of 1100 generations, and selection is not always required for maintenance (Dahlberg and Chao 2003; Harrison et al. 2015; Porse et al. 2016). Importantly, antibiotic concentrations as low as $1 / 1000^{\text {th }}$ the MIC for that drug have been found to select for chromosomal AMR mutants, so the use of low antibiotic concentrations in the various selection conditions will likely be the same for plasmidmediated AMR (Gullberg et al. 2011). Fitness of the evolved populations generally increased over time, and in some cases, the fitness of populations evolved in increasing levels of selection was different than the fitness of those in the LB-only selection environment. Fitness was generally higher after 500 generations of evolution for all three genotypes in the LB and $1 \mathrm{X}$ ancestral MIC competition conditions, but there was no difference in fitness from 0 to 500 generations for the competitions carried out in the 10X ancestral MIC condition. I had predicted that the amount of selection for plasmid-borne resistance would have an impact on fitness, persistence, and the degree of parallel evolution. It was evident that in some cases, at least, that selection environment mattered for the fitness trajectory observed over time, but the plasmid persisted regardless of the selection environment. Parallel evolution was evident in the sequencing data, but occurred regardless of the environment in which the populations were evolved in. The kinds of mutations most frequently observed were related to transcription regulation, which corroborates results found by Harrison et al (2015) and aligns with theory that 
plasmid transcriptional misregulation can be a way that plasmids become costly to their hosts (San Millan and MacLean 2017).

Finally, in my third data chapter, Chapter 5, I constructed six host-plasmid pairs in two strains of E. coli and measured fitness and resistance in twelve environments to assess the presence of gene-by-environment interactions. GxE interactions can mediate the fitness of AMR bacteria, but this has not yet been investigated with respect to plasmids (Clarke et al. 2020). Since plasmid-carrying bacteria can exist in many kinds of environments, understanding the fitness implications that these environmental factors have is essential to combatting AMR in potential reservoirs, like wastewater treatment plants, can have many practical implications. As such, I set out to determine if GxE interactions were evident for plasmids, both in terms of fitness and resistance in the twelve environments. I found that GxE interactions were a significant factor in determining the fitness outcomes of the host-plasmid combinations I tested. Resistance to antibiotics that the plasmid carried resistance determinants for varied between environments, however, these patterns aligned with physiological and chemical factors which influenced the activity of the drugs and their ability to act on the bacterium. This means GxE is a very relevant topic relating to both AMR and plasmids, and further investigations into GxE in these cases for the reduction of AMR pathogens is essential. In my thesis, I aimed to illuminate areas of plasmid biology, particularly as they relate to AMR, because of the public health good that might come from understanding how plasmids are maintained and leveraging this knowledge to reduce plasmid-mediated AMR. Conjugation was found to be an important way for costly plasmids to be maintained when in mixed culture; it is unlikely that a plasmid-carrying bacterium will be 
alone in its environment, so regardless of the cost, this suggests that a plasmid is likely to be maintained over time in some way. I am not the first person to suggest that if conjugation or compensatory evolution is a way that costly plasmids can be maintained, then it is essential to prevent the conjugation of a plasmid into new hosts in the first place (Lopatkin et al. 2017). Research is being done to investigate ways to prevent or reduce the level of plasmid conjugation, and based on my results, I believe that this is an important area to work in (Buckner et al. 2018, 2020; Graf et al. 2019).

However, this does not necessarily provide a simple solution to the problem of plasmid-mediated AMR. Indeed, I have shown that fitness can increase over time, with and without antibiotic selection, and this means that it is less likely these strains would be outcompeted by other bacteria in the same environment. Restriction protocols, as I mentioned in Chapter 1, rely on costly AMR bacteria being outcompeted by susceptible competitors when the use of antibiotics the AMR bacteria has resistance for is restricted for a time (Tang et al. 2017). Ideally, this leverages the fact that many AMR mutations and genes might be costly, and that susceptible competitors will do better when the drug is absent. If the fitness of the AMR pathogen is higher than the susceptible competitor(s), this is unlikely to work, and this means that alternatives to restriction protocols will be necessary for reducing these more fit strains.

My work on GxE might provide a useful avenue for combatting the issue of AMR when fitness might be higher for an AMR bacterium relative to its susceptible competitor(s). Indeed, my measures of fitness in Chapters 3 and $\mathbf{4}$ were done in either LB or LB with kanamycin, and thus the relative fitness values only reflect the fitness in a permissive lab environment. My work in Chapter 5 measured the fitness of six host- 
plasmid combinations in twelve different environments, a combination of different $\mathrm{pH}$, glucose concentrations (low or regular), and the presence/absence of oxygen. I found that there were GxE interactions, such that a combination of these environmental factors and the host-plasmid pair act together to determine fitness. As such, at least in abiotic environments, like wastewater treatment plants or food production plants, we might be able to use some of the information found here and in any follow-up studies, to create plans to reduce AMR bacteria in those environments. This could be done by manipulating the conditions to decrease the fitness of bacteria and use the usual methods of removal (chemical treatments, UV, etc.) in conjunction with competition between susceptible and resistant bacteria, to reduce the prevalence of AMR bacteria that can make it back into the environment.

I think that there are several follow-up experiments that can be informed by the results I have reported here. Primarily, I think it is essential that further studies which investigate the impact that the initial host-plasmid fitness measure has on the strategy (conjugation, compensation, etc.) that might aid in maintaining the plasmid. I measured the fitness of a single plasmid in six strains of $E$. coli, but this plasmid only represents one incompatibility group, and the six hosts are all of the same species, so studies which expand on the type and number of plasmids and represent several important pathogen species will further our understanding in the roles that these strategies play in plasmid maintenance for conjugative plasmids. Similarly, in my long-term selection experiment, I only varied the level of selection, and not the type of selection used when evolving the populations. Since bacteria can face a variety of environments, testing the effect that different environments or factors have on fitness trajectories over time can be useful. 
Finally, as I discussed in Chapter 5, while I measured fitness in twelve environments, I only varied three factors and tested a few levels within these. I think it would be important to test more levels of $\mathrm{pH}$ and glucose concentrations, as well as introduce a micro-aerobic environment. Further, I think it is important to introduce other factors, such as temperature, to determine their importance and utility in a strategy to reduce AMR plasmids in environmental reservoirs. For example, MacFadden et al (2018) reported that the prevalence of AMR increased with local temperature within the United States. This means that warmer ambient temperatures in a given location can lead to increased instances of resistance, a problem that becomes worse when factoring in climate change. Worryingly, they note that resistance to some antibiotics can increase upwards of $10 \%$ if temperatures increase by $10^{\circ} \mathrm{C}$, which they note may be an extreme, but realistic, increase in some parts of the United States in the next century. Similarly, estimates from 28 countries in Europe show that countries with higher ambient minimum temperatures had increased rates of resistance for all drug classes in E. coli and $K$. pneumoniae when compared to countries with cooler temperatures (McGough et al. 2020). Taken together, these results show how important it is to assess temperature, as well as other environmental factors, on AMR over time.

I have answered the questions I outlined at the beginning of this thesis, namely those regarding the long-term impact of fitness on plasmid maintenance strategies, the importance of selection on plasmid evolution, and the role that GxE has on fitness of plasmid-carrying strains. Not only have I helped to expand work that has previously been done in this field, but I have also investigated questions which have not been previously asked. Future studies will be essential to figure out the applicability of my results across 
different hosts and plasmids, and to determine if these trends reflect broad evolutionary patterns or if genetic or environmental interactions will always mediate specific outcomes. Understanding the underlying causes of plasmid fitness and persistence will go a long way in helping scientists formulate adequate responses to the problem of plasmidborn antimicrobial resistance. 


\section{Appendices}

\section{Appendix A : Appendix to Chapter 3}

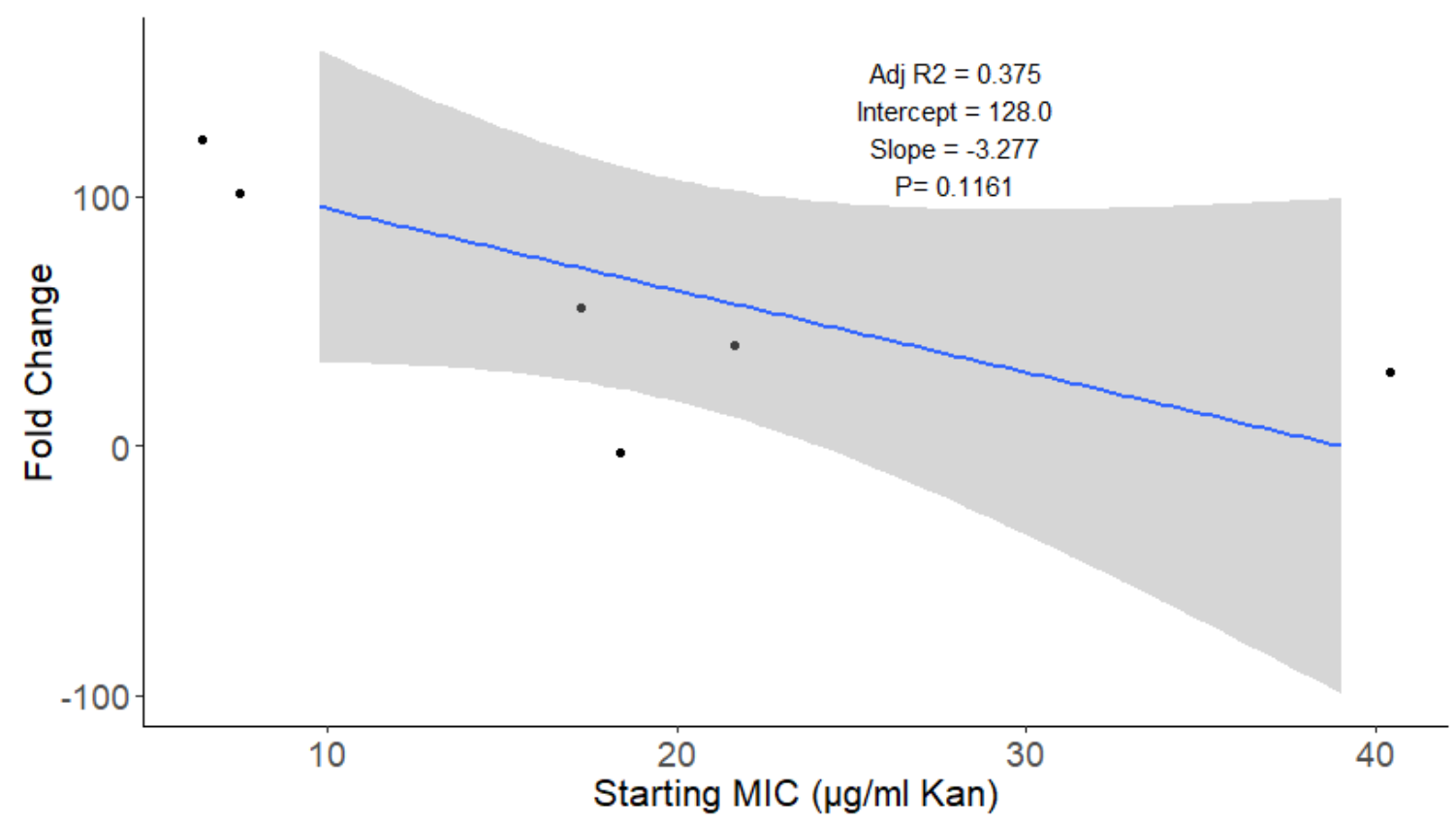

Figure A.1: Fold change in MIC decrease, although not significantly, with starting MIC.

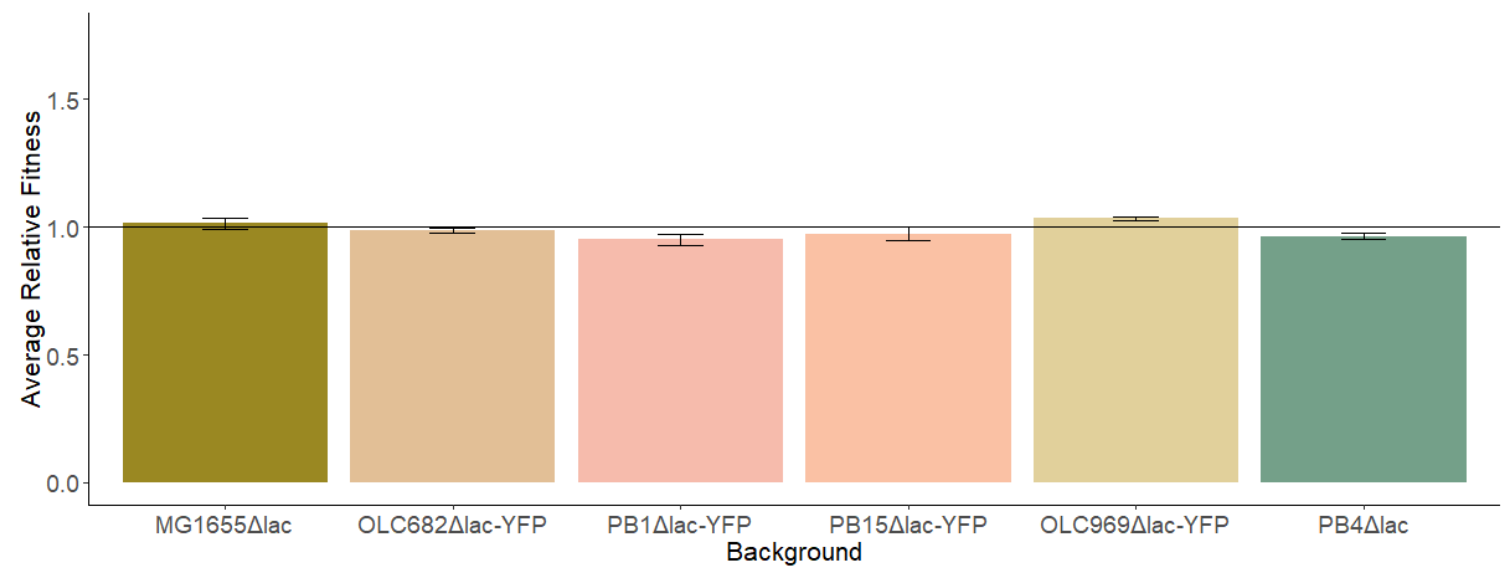

Figure A.2: Effect of genetic marker on recipient fitness. Effect of genetic marker ( $\triangle$ lac or $\Delta l a c$-YFP) in MG1655, PB 1, 4, and 15, OLC682 and OLC969. Results obtained from mixed culture competition assay of the plasmid-free marked strains against their unmarked ancestors. 


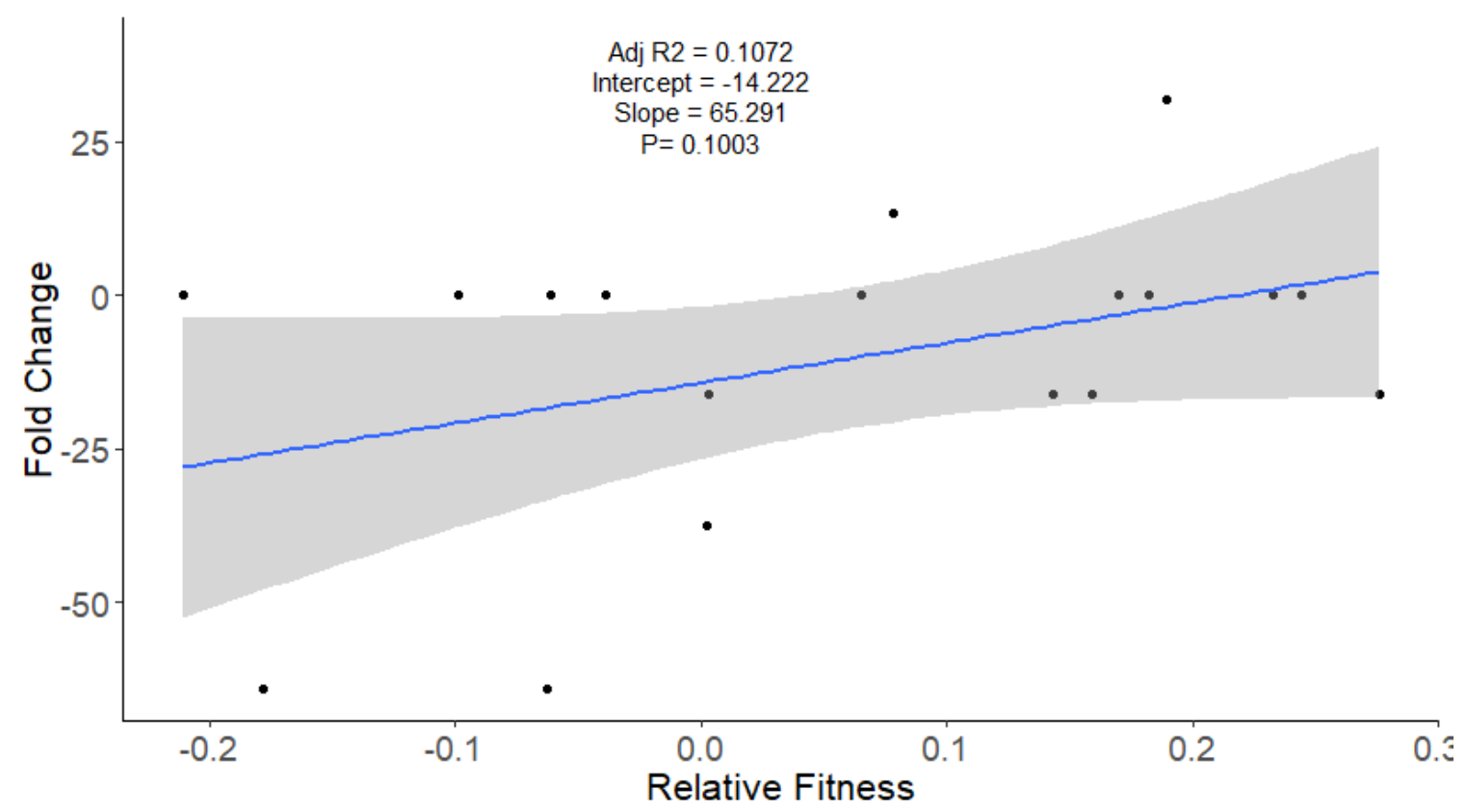

Figure A.3: Comparison of changes in resistance to changes in fitness. There is no correlation between the change in fitness and resistance.

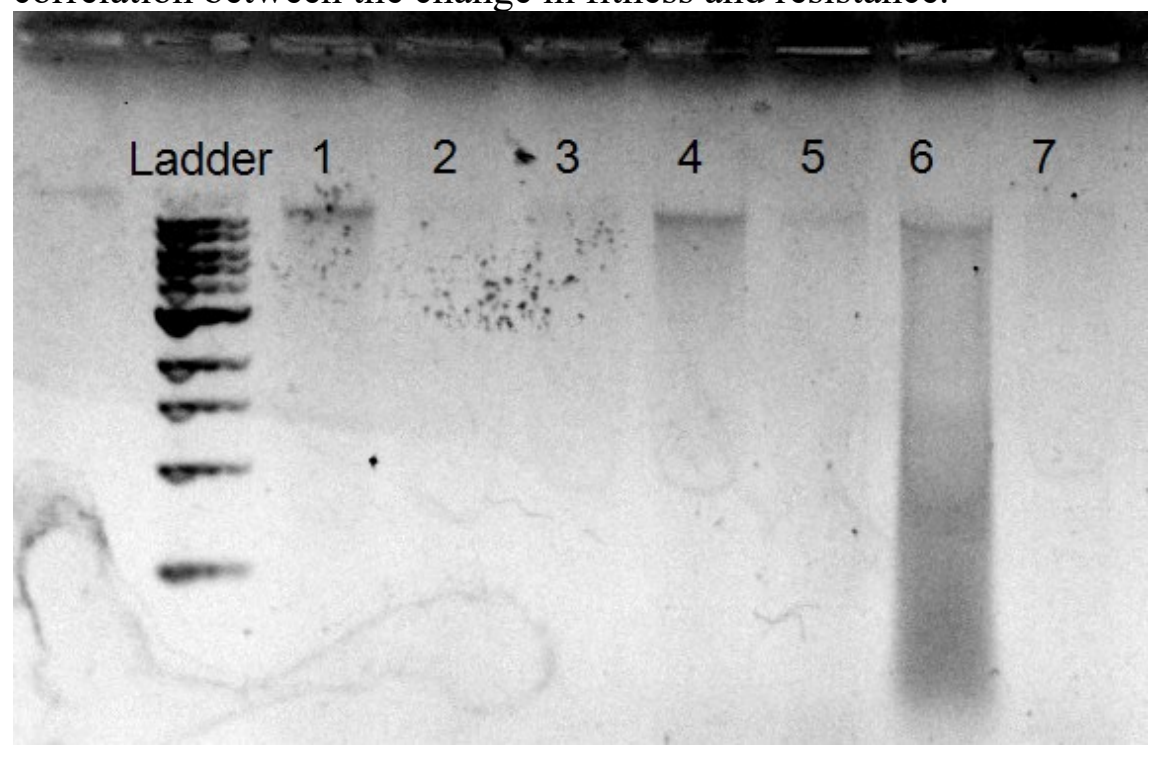

Figure A.4: Plasmid extraction from putative plasmid-free clones following 100 generations of selection in LB medium. Gel is $0.7 \%$ agarose in $1 \mathrm{X}$ TBE with $5 \mu 1$ of Biotium GelRed. Ladder is NEB $1 \mathrm{~kb}$ ladder. Lane 1: evolved MG1655 $\triangle a c+p P B 29$. Lane 2: evolved PB4 $l a c+p P B 29$. Lane 3-5: evolved OLC969 $l a c-Y F P+p P B 29$. Lane 6: Positive control; evolved MG1655 $\Delta l a c+$ pPB29 with normal growth. Lane 7: ancestral MG1655. 


\section{Appendix B}

Supplementary materials from Chapter 4. 


\section{B.1 Sequencing and Parallel Evolution Supplementary Table}

Table B: Raw mutation counts, by type, for all levels (replicate, condition, and genotype).

\begin{tabular}{|c|c|c|c|c|c|c|c|c|c|c|}
\hline \multirow[b]{2}{*}{ Genotype } & \multirow{2}{*}{$\begin{array}{l}\text { Selection } \\
\text { Condition } \\
\end{array}$} & \multirow[b]{2}{*}{ Replicate } & \multirow{2}{*}{$\begin{array}{c}\text { Total } \\
\text { Unique } \\
\end{array}$} & \multirow{2}{*}{$\begin{array}{l}\text { Non-coding } \\
\text { Mutations }\end{array}$} & \multirow[b]{2}{*}{ Total } & \multicolumn{5}{|c|}{ Type of Mutation } \\
\hline & & & & & & Synonymous & Missense & Stop & Frameshift & Intergenic \\
\hline \multirow[t]{9}{*}{ MG1655 } & LB & R1 & 4 & 3 & 7 & 0 & 2 & 0 & 1 & 1 \\
\hline & & $\mathrm{R} 2$ & 5 & 3 & 8 & 0 & 3 & 1 & 0 & 1 \\
\hline & & R3 & 2 & 4 & 6 & 0 & 1 & 1 & 0 & 0 \\
\hline & $\mathrm{Kn} 0.1$ & R1 & 2 & 4 & 6 & 0 & 1 & 0 & 0 & 1 \\
\hline & & $\mathrm{R} 2$ & 1 & 3 & 4 & 0 & 0 & 1 & 0 & 0 \\
\hline & & R3 & 1 & 4 & 5 & 0 & 1 & 0 & 0 & 0 \\
\hline & $\mathrm{Kn} 10$ & R1 & 2 & 3 & 5 & 0 & 1 & 0 & 1 & 0 \\
\hline & & $\mathrm{R} 2$ & 4 & 1 & 5 & 0 & 1 & 2 & 0 & 0 \\
\hline & & R3 & 1 & 3 & 4 & 0 & 0 & 1 & 0 & 0 \\
\hline \multirow[t]{9}{*}{ PB1 } & LB & R1 & 5 & 3 & 8 & 2 & 1 & 1 & 1 & 0 \\
\hline & & R2 & 6 & 4 & 10 & 2 & 2 & 2 & 2 & 0 \\
\hline & & R3 & 8 & 5 & 13 & 4 & 2 & 1 & 1 & 0 \\
\hline & $\mathrm{Kn} 0.1$ & $\mathrm{R} 1$ & 6 & 6 & 12 & 2 & 2 & 0 & 2 & 0 \\
\hline & & $\mathrm{R} 2$ & 5 & 1 & 6 & 2 & 2 & 0 & 1 & 0 \\
\hline & & R3 & 7 & 6 & 13 & 4 & 3 & 0 & 0 & 0 \\
\hline & $\mathrm{Kn} 10$ & $\mathrm{R} 1$ & 5 & 4 & 9 & 2 & 2 & 0 & 1 & 0 \\
\hline & & $\mathrm{R} 2$ & 3 & 4 & 7 & 3 & 0 & 0 & 0 & 0 \\
\hline & & R3 & 7 & 5 & 12 & 2 & 4 & 1 & 0 & 0 \\
\hline \multirow[t]{9}{*}{ OLC969 } & LB & $\mathrm{R} 1$ & 2 & 3 & 5 & 2 & 0 & 0 & 0 & 0 \\
\hline & & $\mathrm{R} 2$ & 4 & 2 & 6 & 3 & 1 & 0 & 0 & 0 \\
\hline & & R3 & 4 & 3 & 7 & 4 & 0 & 0 & 0 & 0 \\
\hline & $\mathrm{Kn} 0.1$ & $\mathrm{R} 1$ & 2 & 2 & 4 & 0 & 2 & 0 & 0 & 0 \\
\hline & & $\mathrm{R} 2$ & 2 & 4 & 6 & 1 & 2 & 0 & 0 & 0 \\
\hline & & R3 & 3 & 1 & 4 & 0 & 2 & 0 & 0 & 0 \\
\hline & $\mathrm{Kn} 10$ & $\mathrm{R} 1$ & 5 & 1 & 6 & 1 & 3 & 0 & 1 & 0 \\
\hline & & $\mathrm{R} 2$ & 2 & 2 & 4 & 0 & 1 & 0 & 1 & 0 \\
\hline & & R3 & 2 & 3 & 5 & 0 & 2 & 0 & 0 & 0 \\
\hline
\end{tabular}




\section{References}

Academies, C. of C. 2019. When antibiotics fail: The expert panel on the potential socioeconomic impacts of antimicrobial resistance in Canada, Council of Canadian Academies.

Alokam, S., S. L. Liu, K. Said, and K. E. Sanderson. 2002. Inversions over the terminus region in Salmonella and Escherichia coli: IS200s as the sites of homologous recombination inverting the chromosome of Salmonella enterica serovar typhi. J. Bacteriol. 184:6190-6197. American Society for Microbiology.

Andrews, S. 2010. FastQC: A Quality Control Tool for High Throughput Sequence Data.

Aslam, B., W. Wang, M. I. Arshad, M. Khurshid, S. Muzammil, M. H. Rasool, M. Atif Nisar, R. F. Alvi, M. A. Aslam, M. Usman Qamar, M. Khalid, F. Salamat, and Z. Baloch. 2018. Infection and Drug Resistance Dovepress Antibiotic resistance: a rundown of a global crisis. , doi: 10.2147/IDR.S173867.

Atanasov, B. P., D. Mustafi, and M. W. Makinen. 2000. Protonation of the $\beta$-lactam nitrogen is the trigger event in the catalytic action of class A $\beta$-lactamases. Proc. Natl. Acad. Sci. U. S. A. 97:3160-3165. Proc Natl Acad Sci U S A.

Aziz, R. K., D. Bartels, A. Best, M. DeJongh, T. Disz, R. A. Edwards, K. Formsma, S. Gerdes, E. M. Glass, M. Kubal, F. Meyer, G. J. Olsen, R. Olson, A. L. Osterman, R. A. Overbeek, L. K. McNeil, D. Paarmann, T. Paczian, B. Parrello, G. D. Pusch, C. Reich, R. Stevens, O. Vassieva, V. Vonstein, A. Wilke, and O. Zagnitko. 2008. The RAST Server: Rapid annotations using subsystems technology. BMC Genomics 9:75. BioMed Central.

Bachmann, B. J. 1972. Pedigrees of some mutant strains of Escherichia coli K-12. 
Bailey, S. F., and T. Bataillon. 2016. Can the experimental evolution programme help us elucidate the genetic basis of adaptation in nature? Mol. Ecol. 25:203-218.

Bailey, S. F., F. Blanquart, T. Bataillon, and R. Kassen. 2017. What drives parallel evolution?: How population size and mutational variation contribute to repeated evolution. BioEssays 39:1-9.

Bailey, S. F., A. Hinz, and R. Kassen. 2014. Adaptive synonymous mutations in an experimentally evolved Pseudomonas fluorescens population. Nat. Commun. 5:1-7. Nature Publishing Group.

Bailey, S. F., N. Rodrigue, and R. Kassen. 2015. The effect of selection environment on the probability of parallel evolution. Mol. Biol. Evol. 32:1436-1448. Oxford University Press.

Baltrus, D. A. 2013. Exploring the costs of horizontal gene transfer. Elsevier.

Bankevich, A., S. Nurk, D. Antipov, A. A. Gurevich, M. Dvorkin, A. S. Kulikov, V. M. Lesin, S. I. Nikolenko, S. Pham, A. D. Prjibelski, A. V. Pyshkin, A. V. Sirotkin, N. Vyahhi, G. Tesler, M. A. Alekseyev, and P. A. Pevzner. 2012. SPAdes: A new genome assembly algorithm and its applications to single-cell sequencing. J. Comput. Biol. 19:455-477. Mary Ann Liebert, Inc.

Basra, P., A. Alsaadi, G. Bernal-Astrain, M. L. O’Sullivan, B. Hazlett, L. M. Clarke, A. Schoenrock, S. Pitre, and A. Wong. 2018. Fitness Tradeoffs of Antibiotic Resistance in Extraintestinal Pathogenic Escherichia coli. Genome Biol. Evol. 10:667-679. Beceiro, A., M. Tomás, and G. Bou. 2013. Antimicrobial resistance and virulence: A successful or deleterious association in the bacterial world? American Society for Microbiology Journals. 
Bennett, P. M. 2008. Plasmid encoded antibiotic resistance: Acquisition and transfer of antibiotic resistance genes in bacteria. Br. J. Pharmacol. 153:347-357.

Bergstrom, C. T., M. Lipsitch, and B. R. Levin. 2000. Natural selection, infectious transfer and the existence conditions for bacterial plasmids. Genetics 155:15051519.

Blair, J. M. A., M. A. Webber, A. J. Baylay, D. O. Ogbolu, and L. J. V Piddock. 2015. Molecular mechanisms of antibiotic resistance. Nat. Rev. Microbiol. 13:42-51. Nature Publishing Group.

Blaser, J., and R. Lüthy. 1988. Comparative study on antagonistic effects of low pH and cation supplementation on in-vitro activity of quinolones and aminoglycosides against Pseudomonas aeruginosa. J. Antimicrob. Chemother. 22:15-22. Oxford Academic.

Bolger, A. M., M. Lohse, and B. Usadel. 2014. Trimmomatic: A flexible trimmer for Illumina sequence data. Bioinformatics 30:2114-2120. Oxford University Press.

Bouma, J. E., and R. E. Lenski. 1988. Evolution of a bacteria/plasmid association. Nature 335:351-352. Nature Publishing Group.

Boyd, B. M., J. M. Allen, N. P. Nguyen, P. Vachaspati, Z. S. Quicksall, T. Warnow, L. Mugisha, K. P. Johnson, and D. L. Reed. 2017. Primates, Lice and Bacteria: Speciation and Genome Evolution in the Symbionts of Hominid Lice. Mol. Biol. Evol. 34:1743-1757.

Brendler, T., L. Reaves, and S. Austin. 2004. Interplay between Plasmid Partition and Postsegregational Killing Systems. J. Bacteriol. 186:2504-2507. J Bacteriol. Buckner, M. M. C., M. L. Ciusa, and L. J. V. Piddock. 2018. Strategies to combat 
antimicrobial resistance: anti-plasmid and plasmid curing. FEMS Microbiol. Rev. 42:781-804.

Buckner, M. M. C., M. Laura Ciusa, R. W. Meek, A. R. Moorey, G. E. McCallum, E. L. Prentice, J. P. Reid, L. J. Alderwick, A. Di Maio, and L. J. V. Piddock. 2020. HIV drugs inhibit transfer of plasmids carrying extended-spectrum $\beta$-lactamase and carbapenemase genes. MBio 11. American Society for Microbiology.

Cairns, J., M. Jalasvuori, V. Ojala, M. Brockhurst, and T. Hiltunen. 2016. Conjugation is necessary for a bacterial plasmid to survive under protozoan predation. Biol. Lett. 12.

Carattoli, A. 2013. Plasmids and the spread of resistance. Urban \& Fischer.

Carroll, A. C., and A. Wong. 2018. Plasmid persistence: costs, benefits, and the plasmid paradox. Can. J. Microbiol. 64:293-304.

Carroll, L. 1909. Through the Looking Glass and What Alice Found There. Dodge Publishing Company, New York.

Castanié-Cornet, M.-P., K. Cam, B. Bastiat, A. Cros, P. Bordes, and C. Gutierrez. 2010. Acid stress response in Escherichia coli: mechanism of regulation of gadA transcription by RcsB and GadE. Nucleic Acids Res. 38:3546-3554. Narnia.

Chandler, C. H., S. Chari, and I. Dworkin. 2013. Does your gene need a background check? How genetic background impacts the analysis of mutations, genes, and evolution.

Chou, H.-H. H., H.-C. C. Chiu, N. F. Delaney, D. Segrè, and C. J. Marx. 2011. Diminishing returns epistasis among beneficial mutations decelerates adaptation. Science (80-. ). 332:1190-1192. 
Clark, D. P., N. J. Pazdernik, and M. R. McGehee. 2018. Molecular biology. Elsevier.

Clarke, L., A. Pelin, M. Phan, and A. Wong. 2020. The effect of environmental heterogeneity on the fitness of antibiotic resistance mutations in Escherichia coli. Evol. Ecol. 34:379-390.

Coates, A. R., G. Halls, and Y. Hu. 2011. Novel classes of antibiotics or more of the same? LINKED ARTICLES. , doi: 10.1111/bph.2011.163.issue-1.

Conlan, S., A. F. Lau, C. Deming, C. D. Spalding, S. Lee-Lin, P. J. Thomas, M. Park, J. P. Dekker, K. M. Frank, T. N. Palmore, and J. A. Segre. 2019. Plasmid Dissemination and Selection of a Multidrug-Resistant Klebsiella pneumoniae Strain during Transplant-Associated Antibiotic Therapy., doi: 10.1128/mBio.

Cooper, A., C. Carter, H. McLeod, P. Sritharan, M. Wright, A. Koziol, S. Tamber, A. Wong, C. Carrillo, and B. Blais. n.d. Whole Genome Sequence Characterization of Carbapenem Resistant Bacteria isolated from Wastewater in Ontario, Canada. FACETS.

Cottell, J. L., M. A. Webber, and L. J. V Piddock. 2012. Persistence of transferable extended-spectrum- $\beta$-lactamase resistance in the absence of antibiotic pressure. Antimicrob. Agents Chemother. 56:4703-4706.

Crozat, E., C. Winkworth, J. Gaffé, P. F. Hallin, M. A. Riley, R. E. Lenski, and D. Schneider. 2010. Parallel genetic and phenotypic evolution of DNA superhelicity in experimental populations of escherichia coli. Mol. Biol. Evol. 27:2113-2128.

Culebras, E., J. L. Martínez, F. Baquero, and J. C. Pérez-Díaz. 1996. pH modulation of aminoglycoside resistance in Staphylococcus epidermidis harbouring 6'-Naminoglycoside acetyltransferase. J. Antimicrob. Chemother. 37:881-889. 
Dahlberg, C., and L. Chao. 2003. Amelioration of the Cost of Conjugative Plasmid Carriage in Eschericha coli K12. Genetics 165:1641-1649. Genetics Society of America.

De Gelder, L., J. M. Ponciano, P. Joyce, and E. M. Top. 2007. Stability of a promiscuous plasmid in different hosts: No guarantee for a long-term relationship. Microbiology 153:452-463.

De Gelder, L., J. J. Williams, J. M. Ponciano, M. Sota, and E. M. Top. 2008. Adaptive plasmid evolution results in host-range expansion of a broad-host-range plasmid. Genetics 178:2179-90. Genetics.

Delcour, A. H. 2009. Outer membrane permeability and antibiotic resistance. NIH Public Access.

DeMars, Z., S. Biswas, R. G. Amachawadi, D. G. Renter, and V. V. Volkova. 2016. Antimicrobial Susceptibility of Enteric Gram Negative Facultative Anaerobe Bacilli in Aerobic versus Anaerobic Conditions. PLoS One 11:e0155599. Public Library of Science.

Di Luca, M. C., V. Sørum, I. Starikova, J. Kloos, N. Hülter, U. Naseer, P. J. Johnsen, and Ø. Samuelsen. 2017. Low biological cost of carbapenemase-encoding plasmids following transfer from Klebsiella pneumoniae to Escherichia coli. J. Antimicrob. Chemother. 72:85-89. Oxford University Press.

Diamond, G., N. Beckloff, A. Weinberg, and K. Kisich. 2009. The Roles of Antimicrobial Peptides in Innate Host Defense. Curr. Pharm. Des. 15:2377-2392. diCenzo, G. C., A. M. MacLean, B. Milunovic, G. B. Golding, and T. M. Finan. 2014. Examination of Prokaryotic Multipartite Genome Evolution through Experimental 
Genome Reduction. PLoS Genet. 10:e1004742. Public Library of Science.

Dimitriu, T., A. C. Matthews, and A. Buckling. 2020. Increased copy number couples the evolution of plasmid horizontal transmission and antibiotic resistance. bioRxiv 2020.08.12.248336.

Dimitriu, T., F. Medaney, E. Amanatidou, J. Forsyth, R. J. Ellis, and B. Raymond. 2019. Negative frequency dependent selection on plasmid carriage and low fitness costs maintain extended spectrum $\beta$-lactamases in Escherichia coli. Sci. Rep. 9.

Dimopoulou, I. D., J. E. Russell, Z. Mohd-Zain, R. Herbert, and D. W. Crook. 2002. Sitespecific recombination with the chromosomal tRNALeu gene by the large conjugative Haemophilus resistance plasmid. Antimicrob. Agents Chemother. 46:1602-1603. American Society for Microbiology Journals.

Dionisio, F., I. C. Conceiçã, A. C. R. Marques, L. Fernandes, and I. Gordo. 2005a. The evolution of a conjugative plasmid and its ability to increase bacterial fitness. Biol. Lett 1:250-252.

Dionisio, F., I. . Conceição, A. C. . Marques, L. Fernandes, and I. Gordo. 2005b. The evolution of a conjugative plasmid and its ability to increase bacterial fitness. Biol. Lett. 1:250-252. Royal Society.

Dmowski, M., and G. Jagura-Burdzy. 2013. Active stable maintenance functions in low copy-number plasmids of Gram-positive bacteria I. partition systems. Polish J. Microbiol. 62:3-16.

Dykhuizen, D. E., and D. L. Hartl. 1983. Selection in Chemostats.

Eberhard, W. G. 1990. Evolution in Bacterial Plasmids and Levels of Selection. Q. Rev. Biol. 65:3-22. 
Eberhard, W. G. 1989. Why do bacterial plasmids carry some genes and not others? Plasmid.

Enne, V. I., P. M. Bennet, D. M. Livermore, and L. M. C. Hall. 2004. Enhancement of host fitness by the sul2-coding plasmid p9123 in the absence of selective pressure. J. Antimicrob. Chemother. 53:958-963.

Enne, V. I., A. A. Delsol, G. R. Davis, S. L. Hayward, J. M. Roe, and P. M. Bennett. 2005. Assessment of the fitness impacts on Escherichia coli of acquisition of antibiotic resistance genes encoded by different types of genetic element. J. Antimicrob. Chemother. 56:544-551.

Evans, D. F., G. Pye, R. Bramley, A. G. Clark, J. Dyson, and J. D. Hardcastle. 1988. Measurement of gastrointestinal $\mathrm{pH}$ profiles in normal ambulant human subjects.

Ferretti, L., B. Schmiegelt, D. Weinreich, A. Yamauchi, Y. Kobayashi, F. Tajima, and G. Achaz. 2016. Measuring epistasis in fitness landscapes: The correlation of fitness effects of mutations. J. Theor. Biol. 396:132-143.

Finn, T. J., S. Shewaramani, S. C. Leahy, P. H. Janssen, and C. D. Moon. 2017. Dynamics and genetic diversification of Escherichia coli during experimental adaptation to an anaerobic environment. PeerJ 2017.

Fischer, E. A., C. M. Dierikx, A. Van Essen-Zandbergen, H. Jw Van Roermund, D. J. Mevius, A. Stegeman, and D. Klinkenberg. 2014. The IncI1 plasmid carrying the bla CTX-M-1 gene persists in in vitro culture of a Escherichia coli strain from broilers.

Forsberg, K. J., S. Patel, T. A. Wencewicz, and G. Dantas. 2015. The Tetracycline Destructases: A Novel Family of Tetracycline-Inactivating Enzymes. Chem. Biol. 22:888-97. NIH Public Access. 
Foucault, M. L., F. Depardieu, P. Courvalin, and C. Grillot-Courvalin. 2010. Inducible expression eliminates the fitness cost of vancomycin resistance in enterococci. Proc. Natl. Acad. Sci. U. S. A. 107:16964-16969. National Academy of Sciences.

Freitas, D. Y., S. Araújo, A. R. C. Folador, R. T. J. Ramos, J. S. N. Azevedo, M. Tacão, A. Silva, I. Henriques, and R. A. Baraúna. 2019. Extended spectrum beta-lactamaseproducing gram-negative bacteria recovered from an amazonian lake near the city of Belém, Brazil. Front. Microbiol. 10. Frontiers Media S.A.

Futuyma, D. J., M. C. Keese, and D. J. Funk. 1995. Genetic Constraints on Macroevolution: The Evolution of Host Affiliation in the Leaf Beetle Genus Ophraella. Evolution (N. Y). 49:797. JSTOR.

Gadgil, M., V. Kapur, and W. S. Hu. 2005. Transcriptional response of Escherichia coli to temperature shift. Biotechnol. Prog. 21:689-699.

Garneau-Tsodikova, S., and K. J. Labby. 2016. Mechanisms of Resistance to Aminoglycoside Antibiotics: Overview and Perspectives. Medchemcomm. 7:11-27.

Ghigo, J. M. 2001. Natural conjugative plasmids induce bacterial biofilm development. Nature 412:442-445. Nature.

Gifford, D. R., E. Moss, and R. C. Maclean. 2016. Environmental variation alters the fitness effects of rifampicin resistance mutations in Pseudomonas aeruginosa. Evolution (N. Y). 70:725-730.

Goltermann, L., L. Good, and T. Bentin. 2013. Chaperonins fight aminoglycosideinduced protein misfolding and promote short-term tolerance in Escherichia coli. J. Biol. Chem. 288:10483-10489. ÂC 2013 ASBMB. Currently published by Elsevier Inc; originally published by American Society for Biochemistry and Molecular 
Biology.

Gordon, D. M. 1992. Rate of plasmid transfer among Escherichia coli strains isolated from natural populations. J. Gen. Microbiol. 138:17-21. J Gen Microbiol.

Graf, F. E., M. Palm, J. Warringer, and A. Farewell. 2019. Inhibiting conjugation as a tool in the fight against antibiotic resistance. Drug Dev. Res. 80:19-23. John Wiley \& Sons, Ltd.

Gullberg, E., S. Cao, O. G. Berg, C. Ilbäck, L. Sandegren, D. Hughes, and D. I. Andersson. 2011. Selection of resistant bacteria at very low antibiotic concentrations. PLoS Pathog. 7:1-9.

Gunsalus, R. P., and S. J. Park. 1994. Aerobic-anaerobic gene regulation in Escherichia coil : control by the ArcAB and Fnr regulons. Res. Microbiol. 145:437-450. Elsevier Masson.

Guyer, M. S., R. R. Reed, J. A. Steitz, and K. B. Low. 1981. Identification of a sexfactor-affinity site in E. coli as gamma delta. Cold Spring Harb. Symp. Quant. Biol. 45 Pt 1:135-140.

Hall, A. R. 2013a. Genotype-by-environment interactions due to antibiotic resistance and adaptation in Escherichia coli. J. Evol. Biol. 26:1655-1664.

Hall, A. R. 2013b. Genotype-by-environment interactions due to antibiotic resistance and adaptation in Escherichia coli. J. Evol. Biol. 26:1655-1664. John Wiley \& Sons, Ltd.

Hall, J. P. J., M. A. Brockhurst, C. Dytham, and E. Harrison. 2017a. The evolution of plasmid stability: Are infectious transmission and compensatory evolution competing evolutionary trajectories? Plasmid 91:90-95. Academic Press Inc. 
Hall, J. P. J., M. A. Brockhurst, C. Dytham, and E. Harrison. 2017b. The evolution of plasmid stability: Are infectious transmission and compensatory evolution competing evolutionary trajectories? Plasmid 91:90-95.

Hall, J. P. J., E. Harrison, A. K. Lilley, S. Paterson, A. J. Spiers, and M. A. Brockhurst. 2015. Environmentally co-occurring mercury resistance plasmids are genetically and phenotypically diverse and confer variable context-dependent fitness effects. Environ. Microbiol. 17:5008-5022. Blackwell Publishing Ltd.

Hall, J. P. J., A. J. Wood, E. Harrison, and M. A. Brockhurst. 2016. Source-sink plasmid transfer dynamics maintain gene mobility in soil bacterial communities. Proc. Natl. Acad. Sci. U. S. A. 113:8260-8265.

Hall, J. P. J., R. C. T. Wright, D. Guymer, E. Harrison, and M. A. Brockhurst. 2020. Extremely fast amelioration of plasmid fitness costs by multiple functionally diverse pathways. , doi: 10.1099/mic.0.000862.

Harrison, E., and M. A. Brockhurst. 2012. Plasmid-mediated horizontal gene transfer is a coevolutionary process. Trends Microbiol.

Harrison, E., C. Dytham, J. P. J Hall, D. Guymer, A. J. Spiers, S. Paterson, and M. A. Brockhurst. 2016. Rapid compensatory evolution promotes the survival of conjugative plasmids. Mob. Genet. Elements 6:3.

Harrison, E., D. Guymer, A. J. Spiers, S. Paterson, M. A. Brockhurst, and M. A. B. Correspondence. 2015. Parallel Compensatory Evolution Stabilizes Plasmids across the Parasitism-Mutualism Continuum In Brief. Curr. Biol. 25:2034-2039. Cell Press.

Harrison, P. W., R. P. J. Lower, N. K. D. Kim, and J. P. W. Young. 2010. Introducing the 
bacterial "chromid": Not a chromosome, not a plasmid. Trends Microbiol. 18:141148. Trends Microbiol.

Hayes, F., and L. Van Melderen. 2011. Toxins-antitoxins: Diversity, evolution and function. Taylor \& Francis.

Heap, J. T., M. Ehsaan, C. M. Cooksley, Y. K. Ng, S. T. Cartman, K. Winzer, and N. P. Minton. 2012. Integration of DNA into bacterial chromosomes from plasmids without a counter-selection marker. Nucleic Acids Res. 40. Nucleic Acids Res.

Helling, R. B., T. Kinney, and J. Adams. 1981. The maintenance of plasmid-containing organisms in populations of Escherichia coli. J. Gen. Microbiol. 123:129-141. J Gen Microbiol.

Henkel, S. G., A. Ter Beek, S. Steinsiek, S. Stagge, K. Bettenbrock, M. J. T. De Mattos, T. Sauter, O. Sawodny, and M. Ederer. 2014. Basic regulatory principles of Escherichia coli's electron transport chain for varying oxygen conditions. PLoS One 9:e107640. Public Library of Science.

Hernández-Arriaga, A. M., W. T. Chan, M. Espinosa, and R. Díaz-Orejas. 2014. Conditional Activation of Toxin-Antitoxin Systems: Postsegregational Killing and Beyond. Microbiol. Spectr. 2. American Society for Microbiology.

Heuer, H., R. E. Fox, and E. M. Top. 2007. Frequent conjugative transfer accelerates adaptation of a broad-host-range plasmid to an unfavorable Pseudomonas putida host. FEMS Microbiol. Ecol. 59:738-748. FEMS Microbiol Ecol.

Hong, H., J. Jung, and W. Park. 2014. Plasmid-Encoded Tetracycline Efflux Pump Protein Alters Bacterial Stress Responses and Ecological Fitness of Acinetobacter oleivorans. PLoS One 9:107716. 
Hooper, D. C., and G. A. Jacoby. 2015. Mechanisms of drug resistance: Quinolone resistance. Ann. N. Y. Acad. Sci. 1354:12-31.

Hülter, N., J. Ilhan, T. Wein, A. S. Kadibalban, K. Hammerschmidt, and T. Dagan. 2017. An evolutionary perspective on plasmid lifestyle modes. Elsevier Ltd.

Humphrey, B., N. R. Thomson, C. M. Thomas, K. Brooks, M. Sanders, A. A. Delsol, J. M. Roe, P. M. Bennett, and V. I. Enne. 2012. Fitness of Escherichia coli strains carrying expressed and partially silent IncN and IncP1 plasmids. BMC Microbiol. 12.

Jackson, R. W., B. Vinatzer, D. L. Arnold, S. Dorus, and J. Murillo. 2011. The influence of the accessory genome on bacterial pathogen evolution. Mob. Genet. Elements 1:55-65. Informa UK Limited.

Jin, Q., and M. F. Kirk. 2018. pH as a Primary Control in Environmental Microbiology: 1. Thermodynamic Perspective. Front. Environ. Sci. 6.

Johnson, C. M., and A. D. Grossman. 2015. Integrative and Conjugative Elements (ICEs): What They Do and How They Work. Annu. Rev. Genet. 49:577-601. Annual Reviews Inc.

Johnson, T. J., R. S. Singer, R. E. Isaacson, J. L. Danzeisen, K. Lang, K. Kobluk, B. Rivet, K. Borewicz, J. G. Frye, M. Englen, J. Anderson, and P. R. Davies. 2015. In Vivo transmission of an incA/C plasmid in Escherichia coli depends on tetracycline concentration, and acquisition of the plasmid results in a variable cost of fitness. Appl. Environ. Microbiol. 81:3561-3570. American Society for Microbiology. Jones, M. E., J. A. Karlowsky, D. C. Draghi, C. Thornsberry, D. F. Sahm, and J. S. Bradley. 2004. Rates of antimicrobial resistance among common bacterial pathogens 
causing respiratory, blood, urine, and skin and soft tissue infections in pediatric patients. Eur. J. Clin. Microbiol. Infect. Dis. 23:445-455. Springer.

Kado, C. I. 2014. Historical Events That Spawned the Field of Plasmid Biology. Microbiol. Spectr. 2. American Society for Microbiology.

Kassen, R. 2014. Experimental Evolution and the Nature of Biodiversity. Roberts and Company Publishers.

Kishony, R., and S. Leibler. 2003. Environmental stresses can alleviate the average deleterious effect of mutations. J. Biol. 2:14.

Knöppel, A., M. Knopp, L. M. Albrecht, E. Lundin, U. Lustig, J. Näsvall, and D. I. Andersson. 2018. Genetic Adaptation to Growth Under Laboratory Conditions in Escherichia coli and Salmonella enterica. Front. Microbiol. 9:756. Frontiers.

Koeppel, A. F., J. O. Wertheim, L. Barone, N. Gentile, D. Krizanc, and F. M. Cohan. 2013. Speedy speciation in a bacterial microcosm: New species can arise as frequently as adaptations within a species. ISME J. 7:1080-1091. ISME J.

Koraimann, G., and M. A. Wagner. 2014. Social behavior and decision making in bacterial conjugation. Frontiers Media S.A.

Kottara, A., J. P. J. Hall, and M. A. Brockhurst. 2020. The proficiency of the original host species determines community-level plasmid dynamics.

Koziolek, M., M. Grimm, D. Becker, V. Iordanov, H. Zou, J. Shimizu, C. Wanke, G. Garbacz, and W. Weitschies. 2015. Investigation of pH and Temperature Profiles in the GI Tract of Fasted Human Subjects Using the Intellicap® System. J. Pharm. Sci. 104:2855-2863. John Wiley and Sons Inc.

Lau, B. T. C., P. Malkus, and J. Paulsson. 2013. New quantitative methods for measuring 
plasmid loss rates reveal unexpected stability. Plasmid 70:353-361. Plasmid.

Lebeuf-Taylor, E., N. McCloskey, S. F. Bailey, A. Hinz, and R. Kassen. 2019. The distribution of fitness effects among synonymous mutations in a gene under directional selection. Elife 8. eLife Sciences Publications Ltd.

Leclercq, R. 2002. Mechanisms of Resistance to Macrolides and Lincosamides: Nature of the Resistance Elements and Their Clinical Implications. Clin. Infect. Dis. 34:482492. Narnia.

Lee, S. W., and G. Edlin. 1985. Expression of tetracycline resistance in pBR322 derivatives reduces the reproductive fitness of plasmid-containing Escherichia coli. Gene 39:173-180. Gene.

Lei, J., L. Sun, S. Huang, C. Zhu, P. Li, J. He, V. Mackey, D. H. Coy, and Q. He. 2019. The antimicrobial peptides and their potential clinical applications.

Lemaire, S., C. Fuda, F. Van Bambeke, P. M. Tulkens, and S. Mobashery. 2008. Restoration of susceptibility of methicillin-resistant Staphylococcus aureus to $\beta$ lactam antibiotics by acidic pH: Role of penicillin-binding protein PBP. J. Biol. Chem. 283:12769-12776. J Biol Chem.

Lenski, R. E., and J. E. Bouma. 1987. Effects of segregation and selection on instability of plasmid pACYC184 in Escherichia coli B. J. Bacteriol. 169:5314-5316. J Bacteriol.

Lili, L. N., N. F. Britton, and E. J. Feil. 2007. The persistence of parasitic plasmids. Genetics 177:399-405. Genetics.

Liu, S. L., and K. E. Sanderson. 1995. The chromosome of Salmonella paratyphi A is inverted by recombination between rrnH and rrnG. J. Bacteriol. 177:6585-6592. 
American Society for Microbiology.

Liu, Y., Y. Huang, Z. Yang, Y. Sun, S. Gong, S. Hou, C. Chen, Z. Li, Q. Liu, Y. Wu, J. Baseman, and G. Zhong. 2014. Plasmid-encoded Pgp3 is a major virulence factor for Chlamydia muridarum to induce hydrosalpinx in mice. Infect. Immun. 82:53275335. American Society for Microbiology.

Löhr, I. H., N. Hülter, E. Bernhoff, P. J. Johnsen, A. Sundsfjord, and U. Naseer. 2015. Persistence of a pKPN3-like CTX-M-15-encoding IncFIIK plasmid in a Klebsiella pneumonia ST17 host during two years of intestinal colonization. PLoS One 10. Public Library of Science.

Lopatkin, A. J., H. R. Meredith, J. K. Srimani, C. Pfeiffer, R. Durrett, and L. You. 2017. Persistence and reversal of plasmid-mediated antibiotic resistance. Nat. Commun. 8:1689. Nature Publishing Group.

MacFadden, D. R., S. F. McGough, D. Fisman, M. Santillana, and J. S. Brownstein. 2018. Antibiotic resistance increases with local temperature. Nat. Clim. Chang. $8: 510-514$.

MacLean, R. C., and A. San Millan. 2015. Microbial Evolution: Towards Resolving the Plasmid Paradox. Cell Press.

Madigan, Bender, Buckley, Sattley, and Stahl. 2018. Brock Biology of Microorganisms, 15th Edition. Pearson.

Manché, K., L. Notley-Mcrobb, and T. Ferenci. 1999. Mutational Adaptation of Escherichia coli to Glucose Limitation Involves Distinct Evolutionary Pathways in Aerobic and Oxygen-Limited Environments.

Markell, J. A. 2017. Accumulation of Single Nucleotide Polymorphism (SNP) Mutations 
in Escherichia coli Grown Under Food Production Conditions and Their Importance in Outbreak Strain Epidemiology.

Martin, G., and T. Lenormand. 2006. THE FITNESS EFFECT OF MUTATIONS ACROSS ENVIRONMENTS: A SURVEY IN LIGHT OF FITNESS LANDSCAPE MODELS. Evolution (N. Y). 60:2413-2427. Wiley.

Martínez-Gómez, K., N. Flores, H. M. Castañeda, G. Martínez-Batallar, G. HernándezChávez, O. T. Ramírez, G. Gosset, S. Encarnación, and F. Bolivar. 2012. New insights into Escherichia coli metabolism: Carbon scavenging, acetate metabolism and carbon recycling responses during growth on glycerol. Microb. Cell Fact. 11:46. BioMed Central.

Masterson, R. V., P. R. Russell, and A. G. Atherly. 1982. Nitrogen fixation (nif) genes and large plasmids of Rhizobium japonicum. J. Bacteriol. 152:928-931. American Society for Microbiology (ASM).

Mavingui, P., M. Flores, X. Guo, G. Dávila, X. Perret, W. J. Broughton, and R. Palacios. 2002. Dynamics of genome architecture in Rhizobium sp. strain NGR234. J. Bacteriol. 184:171-176. J Bacteriol.

McGough, S. F., D. R. MacFadden, M. W. Hattab, K. Mølbak, and M. Santillana. 2020. Rates of increase of antibiotic resistance and ambient temperature in Europe: A cross-national analysis of 28 countries between 2000 and 2016. Eurosurveillance 25:1-12.

Melnyk, A. H., A. Wong, and R. Kassen. 2015. The fitness costs of antibiotic resistance mutations. Evol. Appl. 8:273-283. Wiley/Blackwell (10.1111).

Millan, A. S., J. A. Escudero, D. R. Gifford, Di. Mazel, and R. C. MacLean. 2016. 
Multicopy plasmids potentiate the evolution of antibiotic resistance in bacteria. Nat. Ecol. Evol. 1:1-8. Nature Publishing Group.

Million-Weaver, S., and M. Camps. 2014. Mechanisms of plasmid segregation: Have multicopy plasmids been overlooked? Plasmid 75:27-36. Academic Press Inc.

Modi, R. I., L. H. Castilla, S. Puskas-Rozsa, R. B. Helling, and J. Adams2. 1992. Genetic Changes Accompanying Increased Fitness in Evolving Populations of Escherichia coli.

Munita, J. M., and C. A. Arias. 2016. Mechanisms of Antibiotic Resistance. Microbiol. Spectr. 4.

Naas, T., G. Cuzon, M. V. Villegas, M. F. Lartigue, J. P. Quinn, and P. Nordmann. 2008. Genetic structures at the origin of acquisition of the $\beta$-lactamase blaKPC gene. Antimicrob. Agents Chemother. 52:1257-1263. American Society for Microbiology Journals.

Nemeth, J., G. Oesch, and S. P. Kuster. 2015. Bacteriostatic versus bactericidal antibiotics for patients with serious bacterial infections: systematic review and metaanalysis. J. Antimicrob. Chemother. 70:382-395. Narnia.

Nguyen, F., A. L. Starosta, S. Arenz, D. Sohmen, A. Dönhöfer, and D. N. Wilson. 2014. Tetracycline antibiotics and resistance mechanisms. Biol. Chem. 395.

Nies, D. H. 1999. Microbial heavy-metal resistance. Appl Microbiol Biotechnol.

Nordstrom, K., and S. J. Austin. 1989. Mechanisms that contribute to the stable segregation of plasmids. Annu. Rev. Genet. 23:37-69.

Notley-McRobb, L., A. Death, and T. Ferenci. 1997. The relationship between external glucose concentration and cAMP levels inside Escherichia coli: Implications for 
models of phosphotransferase-mediated regulation of adenylate cyclase. Microbiology 143:1909-1918.

O’Neill, J. 2016. Tackling Drug-Resistant Infections Globally: Final Report and Recommendations.

OECD. 2018. STEMMING THE SUPERBUG TIDE IN CANADA.

Ohsuka, S., Y. Arakawa, T. Horii, H. Ito, and M. Ohta. 1995. Effect of pH on Activities of Novel-Lactamases and-Lactamase Inhibitors against These-Lactamases The effects of acidic conditions on activities of seven-lactamases-TEM-1.

Olvera, L., A. Mendoza-Vargas, N. Flores, M. Olvera, and J. C. Sigala. 2009. Transcription Analysis of Central Metabolism Genes in Escherichia coli. Possible Roles of s 38 in Their Expression, as a Response to Carbon Limitation. PLoS One $4: 7466$.

Orr, H. A. 2005. The probability of parallel evolution. Evolution (N. Y). 59:216-220.

Pacheco, J. O., C. Alvarez-Ortega, M. A. Rico, and J. L. Martínez. 2017. Metabolic compensation of fitness costs is a general outcome for antibiotic- resistant Pseudomonas aeruginosa mutants overexpressing efflux pumps. MBio 8. American Society for Microbiology.

Pagès, J.-M., C. E. James, and M. Winterhalter. 2008. The porin and the permeating antibiotic: a selective diffusion barrier in Gram-negative bacteria. , doi: 10.1038/nrmicro1994.

Pal, C., J. Bengtsson-Palme, E. Kristiansson, and D. G. J. Larsson. 2015. Co-occurrence of resistance genes to antibiotics, biocides and metals reveals novel insights into their co-selection potential. BMC Genomics 16:964. BioMed Central. 
Peña-Miller, R., R. Rodríguez-González, R. C. MacLean, and A. San Millan. 2015. Evaluating the effect of horizontal transmission on the stability of plasmids under different selection regimes. Mob. Genet. Elements 5:29-33. Taylor and Francis Inc.

Peterson, E., and P. Kaur. 2018. Antibiotic resistance mechanisms in bacteria: Relationships between resistance determinants of antibiotic producers, environmental bacteria, and clinical pathogens. Front. Microbiol. 9:1-21.

Pinto, U. M., K. M. Pappas, and S. C. Winans. 2012. The ABCs of plasmid replication and segregation. Nat. Rev. Microbiol. 10:755-765. Nature Publishing Group.

Poirel, L., V. Cattoir, and P. Nordmann. 2012. Plasmid-Mediated Quinolone Resistance; Interactions between Human, Animal, and Environmental Ecologies. Front. Microbiol. 3:24. Frontiers Research Foundation.

Popov, M., S. Petrov, K. Kirilov, G. Nacheva, and I. Ivanov. 2009. Segregational instability in e. Coli of expression plasmids carrying human interferon gamma gene and its 3'-end truncated variants. Biotechnol. Biotechnol. Equip. 23:840-843. Taylor \& Francis.

Pormohammad, A., M. J. Nasiri, and T. Azimi. 2019. Prevalence of antibiotic resistance in escherichia coli strains simultaneously isolated from humans, animals, food, and the environment: A systematic review and meta-analysis.

Porse, A., K. Schønning, C. Munck, and M. O. A. Sommer. 2016. Survival and Evolution of a Large Multidrug Resistance Plasmid in New Clinical Bacterial Hosts. Mol. Biol. Evol. 33:2860-2873. Oxford University Press.

Public Health Agency of Canada. 2018. Canadian Antimicrobial Resistance Surveillance System (CARSS): Update 2018. 
Ramirez, M. S., N. Nikolaidis, and M. E. Tolmasky. 2013. Rise and dissemination of aminoglycoside resistance: The aac $\left(6^{\prime}\right)$-Ib paradigm. Frontiers Research Foundation.

Ramirez, M. S., G. M. Traglia, D. L. Lin, T. Tran, and M. E. Tolmasky. 2014. PlasmidMediated Antibiotic Resistance and Virulence in Gram-Negatives: the Klebsiella pneumoniae Paradigm. Microbiol. Spectr. 2. American Society for Microbiology.

Rankin, D. J., E. P. C. Rocha, and S. P. Brown. 2011. What traits are carried on mobile genetic elements, and why. Heredity (Edinb).

Reinikainen, P., J. Olkku, P. Markkanen, and A. Holmberg. 1985. DETERMINATION OF pH AND TEMPERATURE PROFILES FOR THE GROWTH OF ESCHERICHIA COLI. IFAC Proc. Vol. 18:219-224. Elsevier.

Relman, D. A., and M. Lipsitch. 2018. Microbiome as a tool and a target in the effort to address antimicrobial resistance. National Academy of Sciences.

Reygaert, W. C. 2018. An overview of the antimicrobial resistance mechanisms of bacteria. AIMS Microbiol. 4:482-501.

Rice, L. B. 2012. Mechanisms of resistance and clinical relevance of resistance to $\beta$ lactams, glycopeptides, and fluoroquinolones. Mayo Clin. Proc. 87:198-208. Mayo Foundation.

Roberts, A. P., and P. Mullany. 2009. A modular master on the move: the Tn916 family of mobile genetic elements. Elsevier Current Trends.

Rozwandowicz, M., M. S. M. Brouwer, J. Fischer, J. A. Wagenaar, B. Gonzalez-Zorn, B. Guerra, D. J. Mevius, and J. Hordijk. 2018. Plasmids carrying antimicrobial resistance genes in Enterobacteriaceae. J. Antimicrob. Chemother. 73:1121-1137. Narnia. 
Russell, J. B., and G. M. Cook. 1995. Energetics of Bacterial Growth: Balance of Anabolic and Catabolic Reactions.

Saltikov, C. W., and D. K. Newman. 2003. Genetic identification of a respiratory arsenate reductase. Proc. Natl. Acad. Sci. U. S. A. 100:10983-10988. National Academy of Sciences.

San Millan, A., K. Heilbron, and R. C. MacLean. 2014a. Positive epistasis between coinfecting plasmids promotes plasmid survival in bacterial populations. ISME J. 8:601-612. Nature Publishing Group.

San Millan, A., and R. C. MacLean. 2017. Fitness Costs of Plasmids: a Limit to Plasmid Transmission. Microbiol. Spectr. 5.

San Millan, A., R. Peña-Miller, M. Toll-Riera, Z. V. Halbert, A. R. McLean, B. S. Cooper, and R. C. Maclean. 2014b. Positive selection and compensatory adaptation interact to stabilize non-transmissible plasmids. Nat. Commun. 5:5208. Nature Publishing Group.

San Millan, A., M. Toll-Riera, Q. Qi, and R. C. MacLean. 2015. Interactions between horizontally acquired genes create a fitness cost in Pseudomonas aeruginosa. Nat. Commun. 6. Nature Publishing Group.

Schlessinger, D. 1988. Failure of Aminoglycoside Antibiotics to Kill Anaerobic, Low$\mathrm{pH}$, and Resistant Cultures.

Schluter, D., E. A. Clifford, M. Nemethy, and J. S. McKinnon. 2004. Parallel evolution and inheritance of quantitative traits. Am. Nat. 163:809-822. The University of Chicago Press .

Schrag, S. J., V. Perrot, and B. R. Levin. 1997. Adaptation to the fitness costs of 
antibiotic resistance in Escherichia coli. Proc. R. Soc. London. Ser. B Biol. Sci. 264:1287-1291. Royal Society.

Seemann, T. 2015. Snippy: Rapid haploid variant calling and core genome alignment.

Shehata, T. E., and A. G. Marr. 1971. Effect of nutrient concentration on the growth of Escherichia coli. J. Bacteriol. 107:210-216.

Sherratt, D. J. 1974. Bacterial plasmids. Cell.

Shidore, T., and L. R. Triplett. 2017. Toxin-Antitoxin Systems: Implications for Plant Disease. Annual Reviews Inc.

Shintani, M., Z. K. Sanchez, and K. Kimbara. 2015. Genomics of microbial plasmids: classification and identification based on replication and transfer systems and host taxonomy. Front. Microbiol. 6:242. Frontiers.

Silva, F., J. A. Queiroz, and F. C. Domingues. 2012. Evaluating metabolic stress and plasmid stability in plasmid DNA production by Escherichia coli.

Simpkin, V. L., M. J. Renwick, R. Kelly, and E. Mossialos. 2017. Incentivising innovation in antibiotic drug discovery and development: Progress, challenges and next steps. Nature Publishing Group.

Sköld, O. 2000. Sulfonamide resistance: mechanisms and trends. Drug Resist. Updat. 3:155-160. Churchill Livingstone.

Slater, F. R., M. J. Bailey, A. J. Tett, and S. L. Turner. 2008. Progress towards understanding the fate of plasmids in bacterial communities. Pp. 3-13 in FEMS Microbiology Ecology.

Sota, M., Yano, Hirokazu, J. Hughes, G. W. Daughdrill, Z. Abdo, L. J. Forney, and E. M. Top. 2010. Shifts in Host Range of a Promiscuous Plasmid through Parallel 
Evolution of its Replication Initiation Protein. ISME J. 4:1568-1580.

Stalder, T., L. M. Rogers, C. Renfrow, H. Yano, Z. Smith, and E. M. Top. 2017.

Emerging patterns of plasmid-host coevolution that stabilize antibiotic resistance.

Sci. Rep. 7:4853. Nature Publishing Group.

Stalder, T., and E. Top. 2016. Plasmid transfer in biofilms: A perspective on limitations and opportunities. Nature Publishing Group.

Starikova, I., M. Al-Haroni, G. Werner, A. P. Roberts, V. Sørum, K. M. Nielsen, and P. J. Johnsen. 2013. Fitness costs of various mobile genetic elements in enterococcus faecium and enterococcus faecalis. J. Antimicrob. Chemother. 68:2755-2765.

Stevenson, C., J. P. J. Hall, E. Harrison, A. J. Wood, and M. A. Brockhurst. 2017. Gene mobility promotes the spread of resistance in bacterial populations. ISME J. 11:1930-1932.

Stewart, V., Y. Lu, and A. J. Darwin. 2002. Periplasmic nitrate reductase (NapABC enzyme) supports anaerobic respiration by Escherichia coli K-12. J. Bacteriol. 184:1314-1323. American Society for Microbiology.

Svara, F., and D. J. Rankin. 2011. The evolution of plasmid-carried antibiotic resistance. BMC Evol. Biol. 11. BMC Evol Biol.

Tang, K. L., N. P. Caffrey, D. B. Nóbrega, S. C. Cork, P. E. Ronksley, H. W. Barkema, A. J. Polachek, H. Ganshorn, N. Sharma, J. D. Kellner, and W. A. Ghali. 2017. Restricting the use of antibiotics in food-producing animals and its associations with antibiotic resistance in food-producing animals and human beings: a systematic review and meta-analysis. Lancet Planet. Heal. 1:e316-e327. Elsevier B.V.

Tenover, F. C. 2006. Mechanisms of Antimicrobial Resistance in Bacteria. Am. J. Med. 
119:S3-S10.

The Council of the Canadian Academies. 2019. When Antibiotics Fail: The Expert Panel on the Potential Socio-Economic Impacts of Antimicrobial Resistance in Canada.

Tiso, M., and A. N. Schechter. 2015. Nitrate reduction to nitrite, nitric oxide and ammonia by gut bacteria under physiological conditions. PLoS One 10:1-18.

Travis, A., O. Chernova, V. Chernov, and R. Aminov. 2018. Antimicrobial drug discovery: lessons of history and future strategies. Taylor and Francis Ltd.

Tsang, J. 2017. Bacterial plasmid addiction systems and their implications for antibiotic drug development. Postdoc J. 5:3. Postdoc Journal.

Tweeddale, H., L. Notley-Mcrobb, and T. Ferenci. 1998. Effect of Slow Growth on Metabolism of Escherichia coli, as Revealed by Global Metabolite Pool ("Metabolome") Analysis Downloaded from.

Unden, G., and M. Trageser. 1991. Oxygen regulated gene expression in Escherichia coli: Control of anaerobic respiration by the FNR protein. Antonie Van Leeuwenhoek $59: 65-76$.

Valenzuela, M. S., E. V. Ikpeazu, and K. A. I. Siddiqui. 1996. E. Coli growth inhibition by a high copy number derivative of plasmid pBR322. Biochem. Biophys. Res. Commun. 219:876-883. Academic Press Inc.

Valle, A. A., R. León-Sampedro, J. Rodríguez-Beltrán, J. DelaFuente, M. HernándezGarcía, P. Ruiz-Garbajosa, R. Cantón, R. Peña-Miller, Á. S. Millán, A. Alonso-del Valle, R. León-Sampedro, J. Rodríguez-Beltrán, M. Hernández-García, P. RuizGarbajosa, R. Cantón, Á. San Millán, M. Spain, and R. Peña-Miller. 2020. The distribution of plasmid fitness effects explains plasmid persistence in bacterial 
communities. bioRxiv 2020.08.01.230672. bioRxiv.

Van Valen, L. 1977. The Red Queen. Am. Soc. Nat. 111:809-810.

Via, S., and R. Lande. 1985. Genotype-Environment Interaction and the Evolution of Phenotypic Plasticity. Evolution (N. Y). 39:505. JSTOR.

Vliegenthart, J. S., P. A. G. Ketelaar-van Gaalen, J. Eelhart, and J. A. M. van de Klundert. 1991. Localisation of the aminoglycoside-(3)-N-acetyltransferase isoenzyme II in Escherichia coli. FEMS Microbiol. Lett. 81:101-105. No longer published by Elsevier.

Vogwill, T., and R. C. MacLean. 2015. The genetic basis of the fitness costs of antimicrobial resistance: a meta-analysis approach. Evol. Appl. 8:284-295. Wiley/Blackwell (10.1111).

Von Wulffen, J., O. Sawodny, R. Feuer, R. Takors, M. Löffler, J. Simen, M. Ederer, J. Lischke, S. Kunz, O. Rieß, K. Schäferhoff, G. Sprenger, and N. Trachtmann. 2016. Transition of an anaerobic Escherichia coli culture to aerobiosis: Balancing mRNA and protein levels in a demand-directed dynamic flux balance analysis. PLoS One $11: 1-17$.

Wan, Z., J. Varshavsky, S. Teegala, J. McLawrence, and N. L. Goddard. 2011. Measuring the rate of conjugal plasmid transfer in a bacterial population using quantitative PCR. Biophys. J. 101:237-244. Biophys J.

Wang, Y. 2017. Spatial distribution of high copy number plasmids in bacteria. Plasmid $91: 2-8$.

Weinreich, D. M., N. F. Delaney, M. A. DePristo, and D. L. Hartl. 2006. Darwinian evolution can follow only very few mutational paths to fitter proteins. Science (80-. 
). 312:111-114.

WHO. 2018. Antimicrobial resistance.

Wong, A. 2017. Epistasis and the evolution of antimicrobial resistance. Frontiers Media SA.

Wong, A., and K. Seguin. 2015. Effects of genotype on rates of substitution during experimental evolution. Evolution (N. Y). 69:1772-1785. Society for the Study of Evolution.

Woods, R., D. Schneider, C. L. Winkworth, M. A. Riley, and R. E. Lenski. 2006. Tests of parallel molecular evolution in a long-term experiment with Escherichia coli.

Wu, Y., R. Z. Aandahl, and M. M. Tanaka. 2015. Dynamics of bacterial insertion sequences: Can transposition bursts help the elements persist? Theories and models. BMC Evol. Biol. 15. BioMed Central Ltd.

Wünsche, A., D. M. Dinh, R. S. Satterwhite, C. D. Arenas, D. M. Stoebel, and T. F. Cooper. 2017. Diminishing-returns epistasis decreases adaptability along an evolutionary trajectory. Nat. Ecol. Evol. 1:61.

Yang, L., K. Wang, H. Li, J. D. Denstedt, and P. A. Cadieux. 2014. The influence of urinary $\mathrm{pH}$ on antibiotic efficacy against bacterial uropathogens. Urology 84:731.e1731.e7. Elsevier Inc.

Yano, H., K. Wegrzyn, W. Loftie-Eaton, J. Johnson, G. E. Deckert, L. M. Rogers, I. Konieczny, and E. M. Top. 2016. Evolved plasmid-host interactions reduce plasmid interference cost. Mol. Microbiol. 101:743-756. John Wiley \& Sons, Ltd (10.1111).

Zahrl, D., M. Wagner, K. Bischof, and G. Koraimann. 2006. Expression and assembly of a functional type IV secretion system elicit extracytoplasmic and cytoplasmic stress 
responses in Escherichia coli. J. Bacteriol. 188:6611-6621. J Bacteriol.

Zhang, X. X., T. Zhang, and H. H. P. Fang. 2009. Antibiotic resistance genes in water environment. 